\title{
The MicroBooNE Technical Design Report
}




\section{Contents}

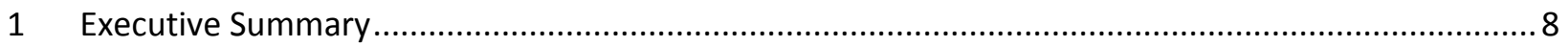

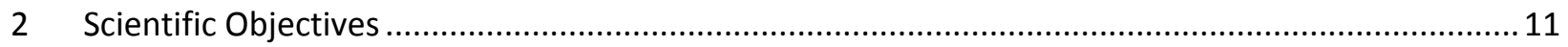

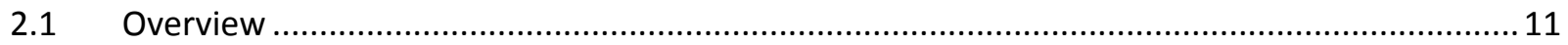

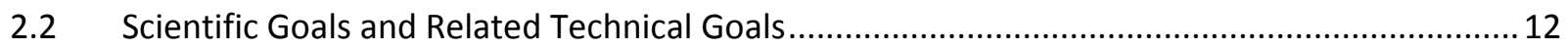

2.2.1 The MiniBooNE Low Energy Excess …......................................................................... 12

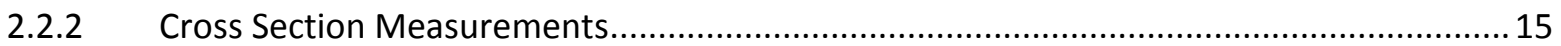

2.2.3 Supernova Detection and Proton Decay Background Measurements ..............................17

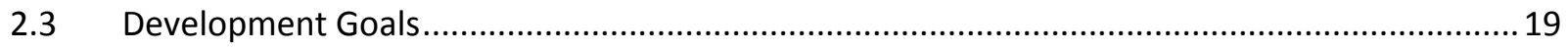

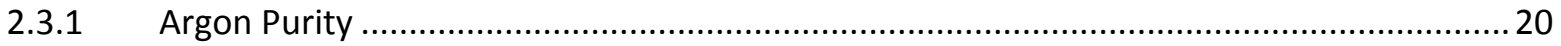

2.3.2 Large Scale Cryogenic Low-noise Electronics................................................................ 20

2.3.3 MicroBooNE Software Development.............................................................................. 20

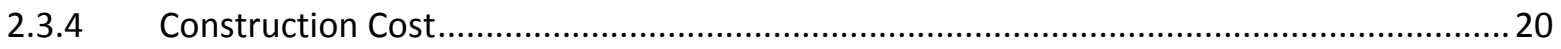

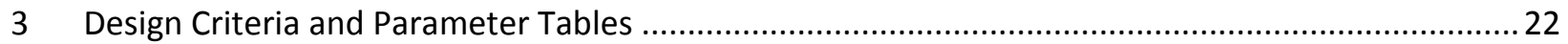

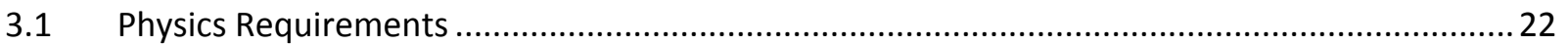

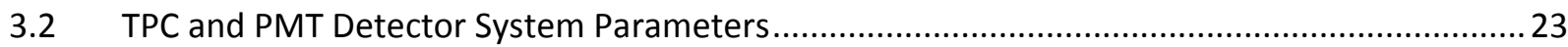

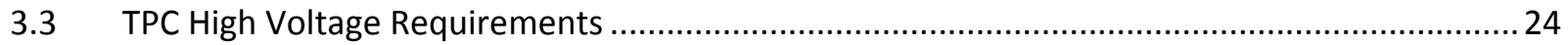

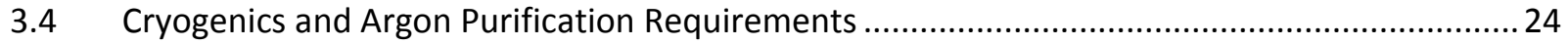

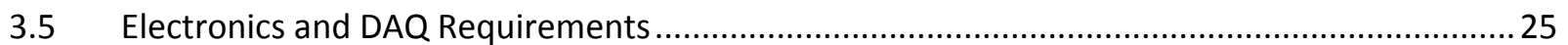

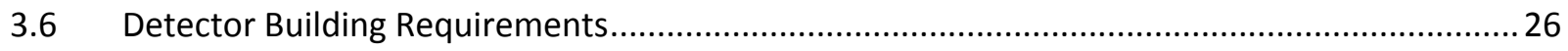

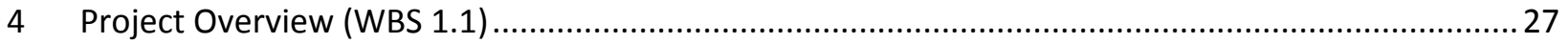

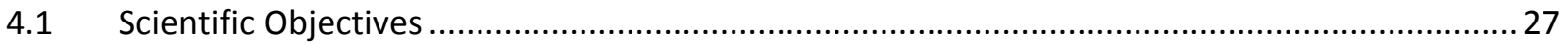

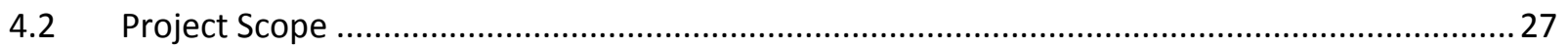

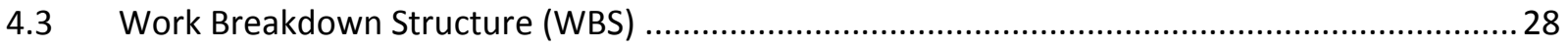

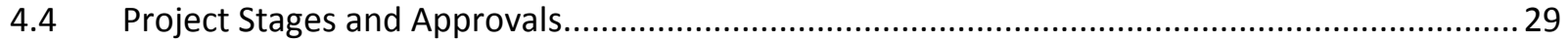

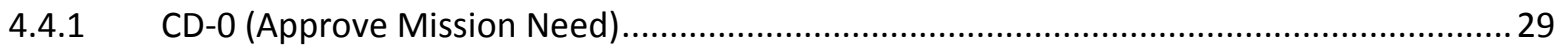

4.4.2 CD-1 (Approve Alternative Selection and Cost Range) …................................................29

4.4.3 CD-2 (Approve Performance Baseline) and CD-3 (Approve Start of Construction) .............30

4.4.4 CD-4 (Approve Project Closeout) ……........................................................................ 30

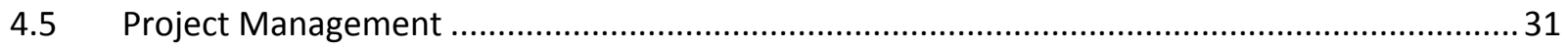




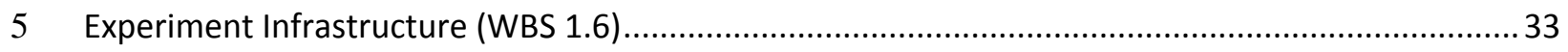

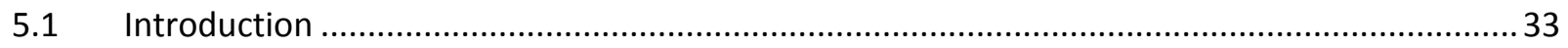

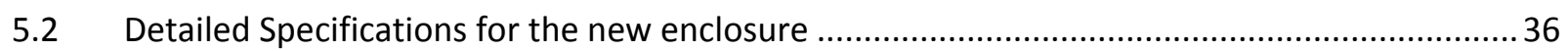

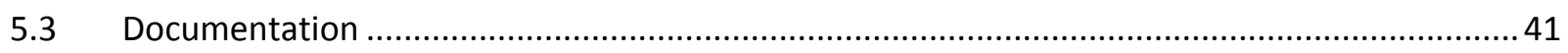

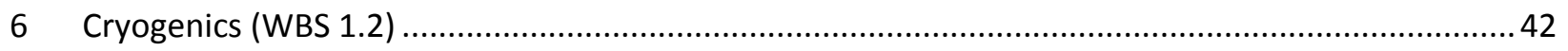

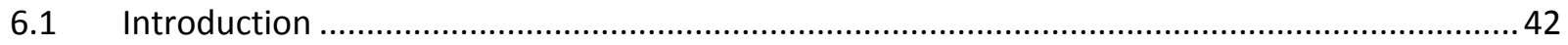

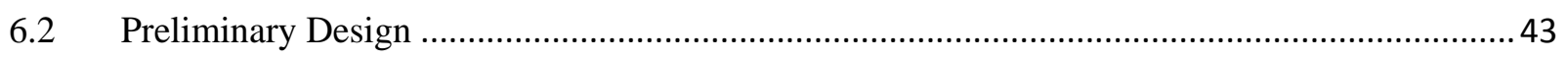

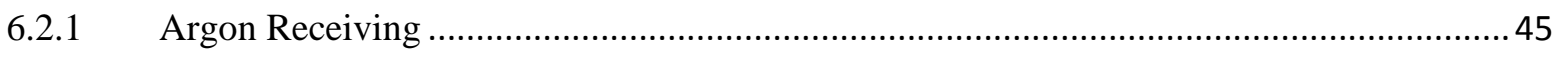

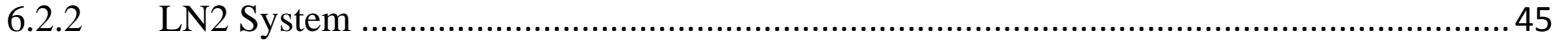

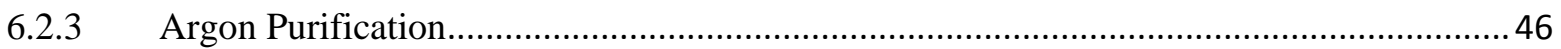

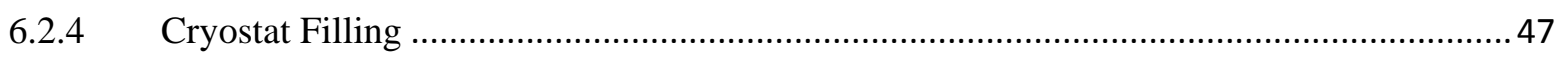

6.2.5 Cryostat Controls and Monitors ................................................................................ 48

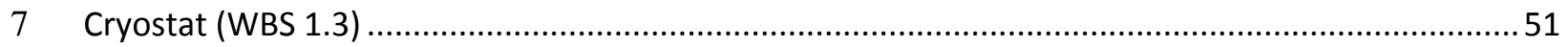

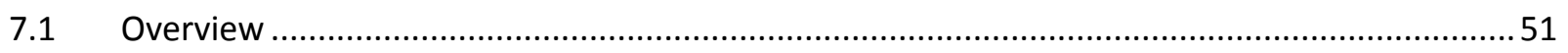

7.1.1 Cryostat Mechanical Design Considerations................................................................. 52

7.1.2 Achieving Acceptably Low Heat Leak ........................................................................... 53

7.1.3 Access, Internal Support, and Interface Considerations ................................................. 54

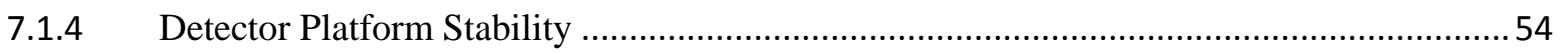

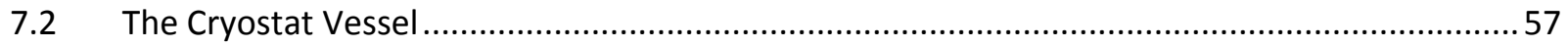

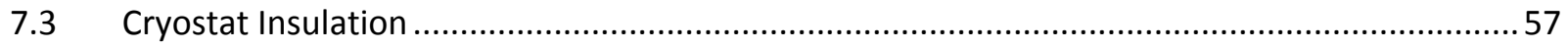

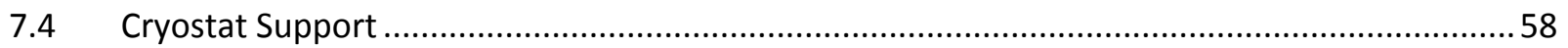

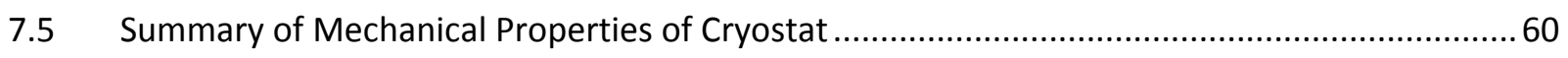

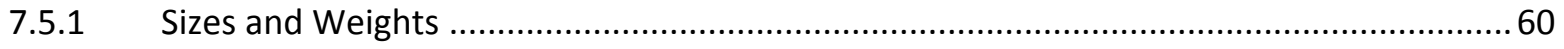

7.5.2 Deflection and Stress of Fully Loaded Vessel............................................................... 60

7.5.3 Deflection and Stress of Saddles Supporting the Fully Loaded Vessel ............................60

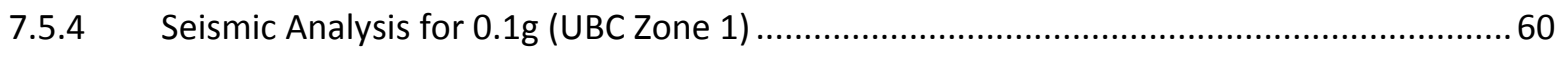

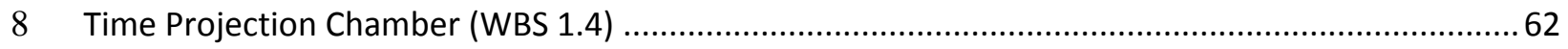

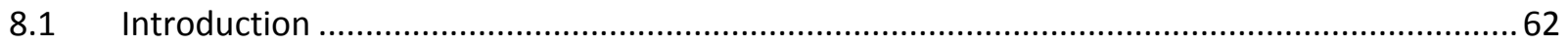

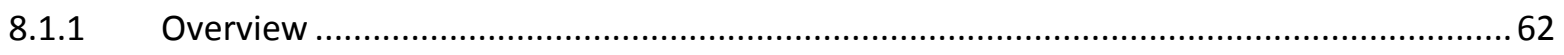

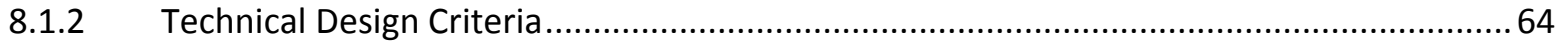

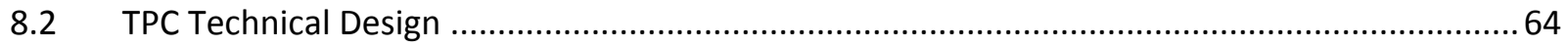

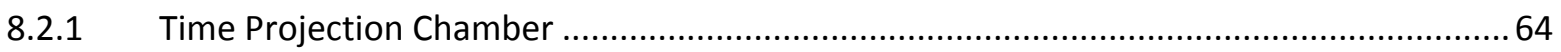




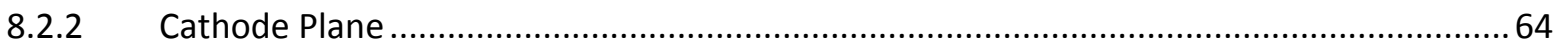

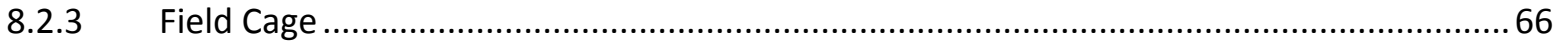

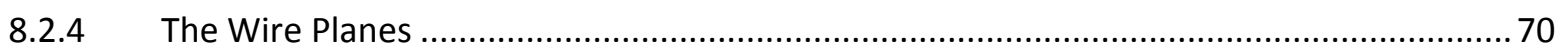

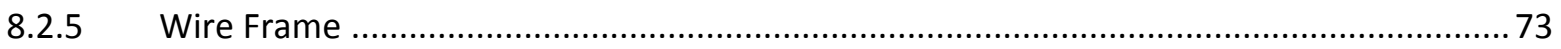

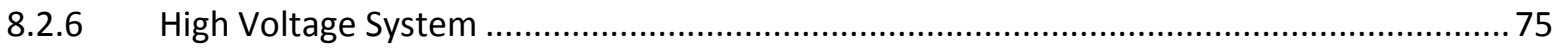

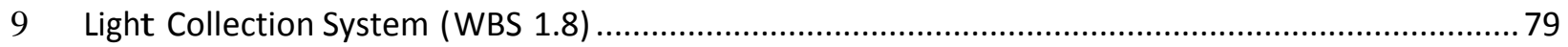

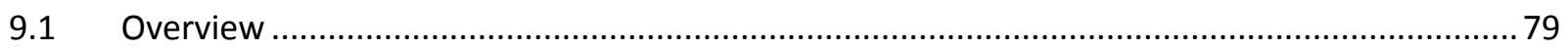

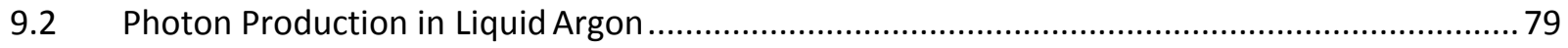

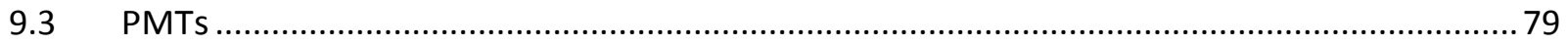

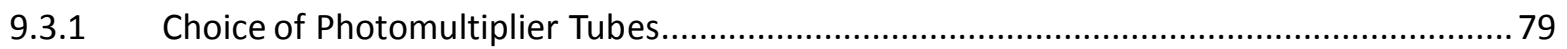

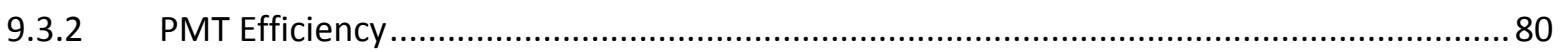

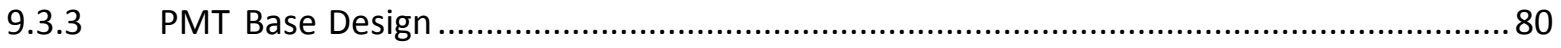

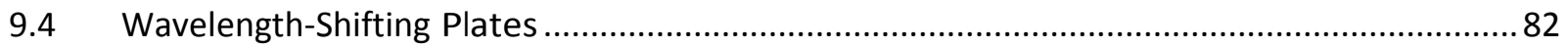

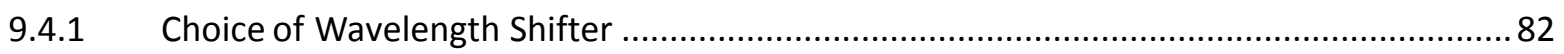

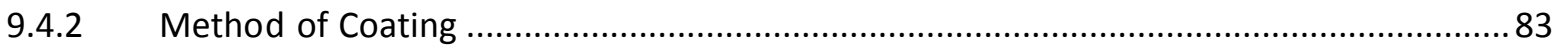

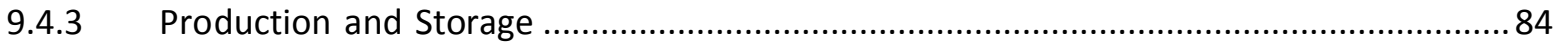

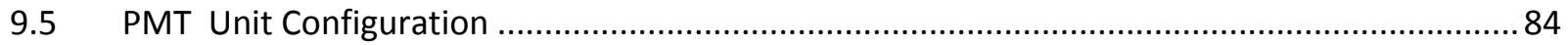

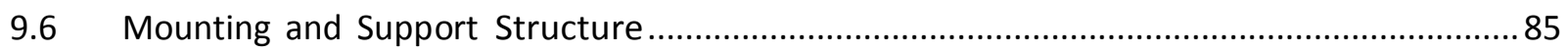

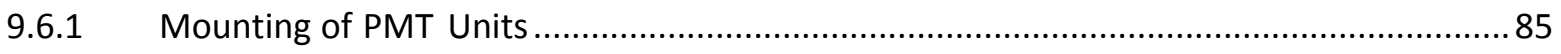

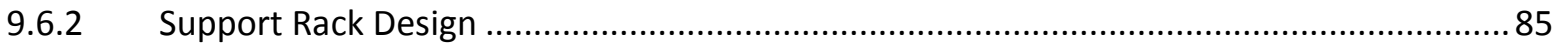

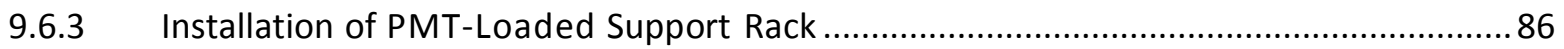

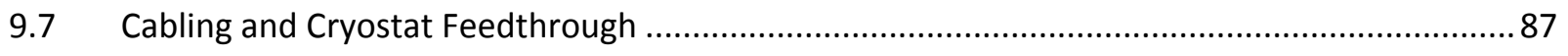

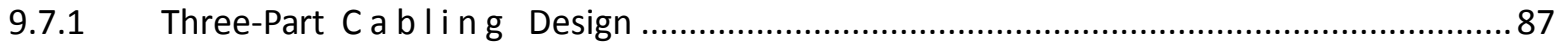

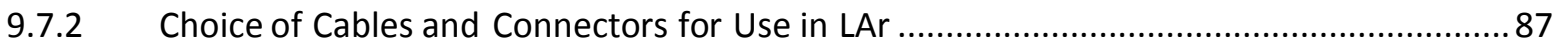

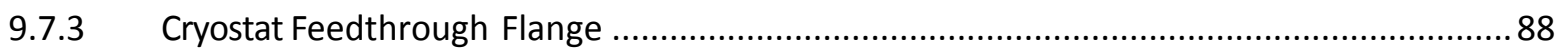

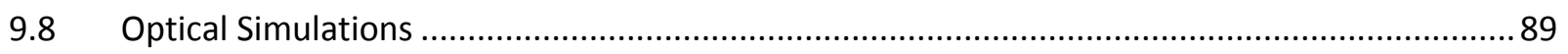

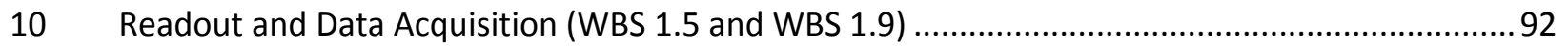

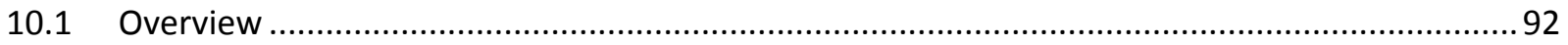

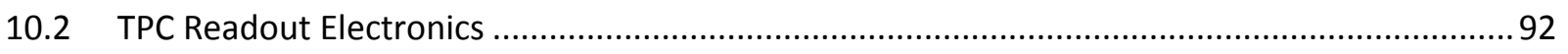

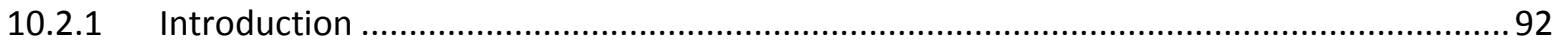

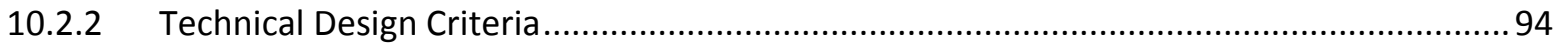

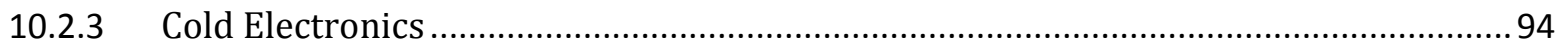




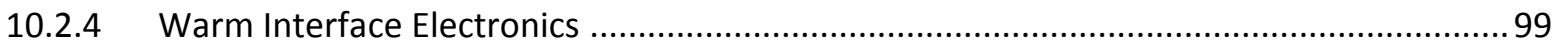

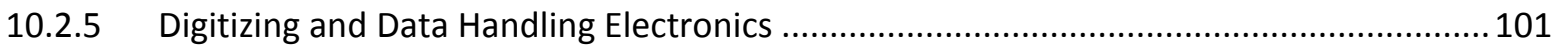

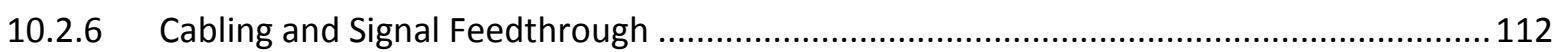

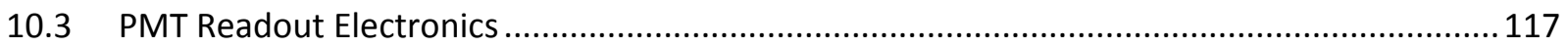

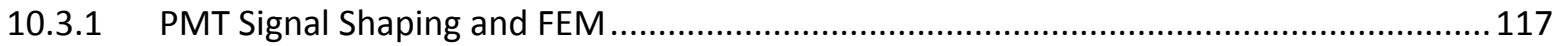

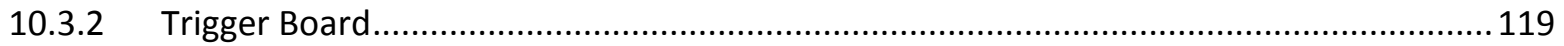

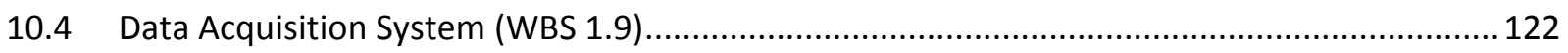

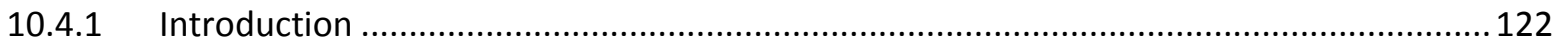

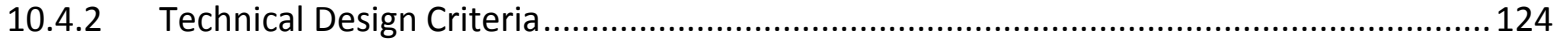

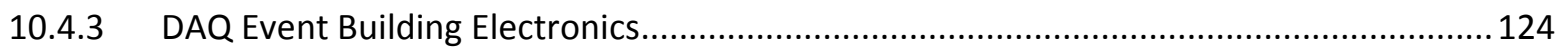

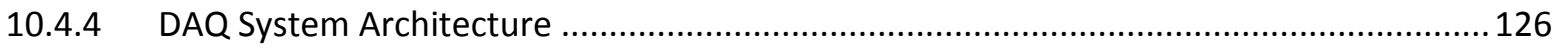

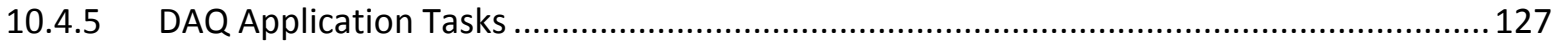

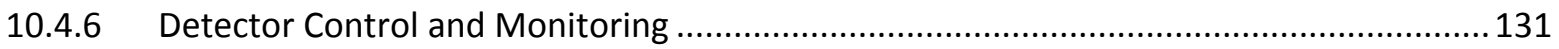

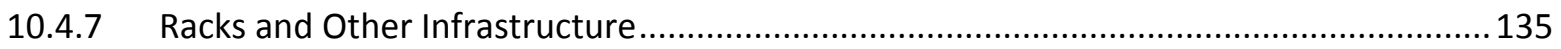

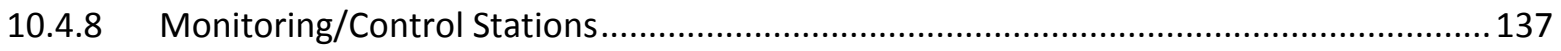

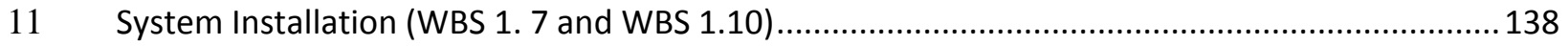

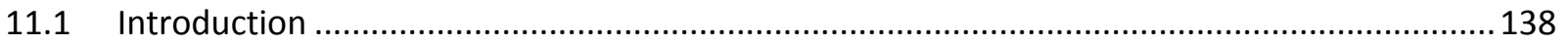

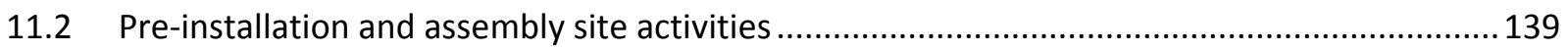

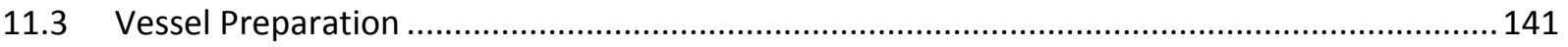

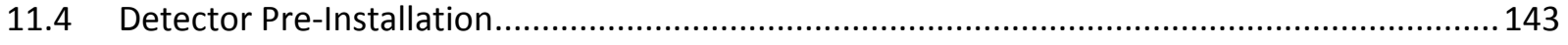

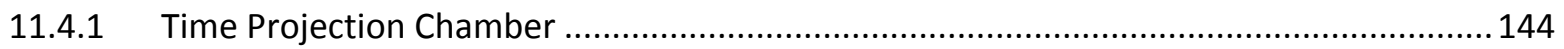

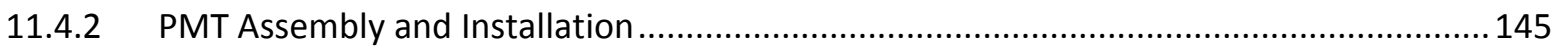

11.5 Installation in Experimental Hall .................................................................................... 147

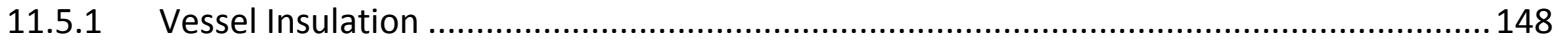

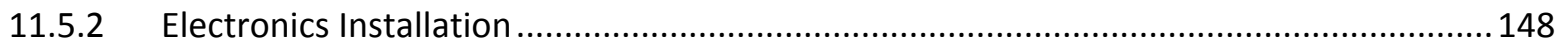

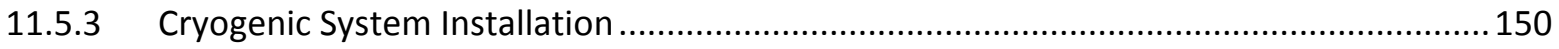

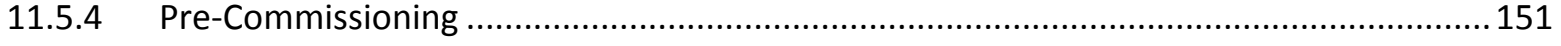

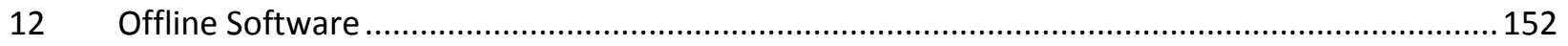

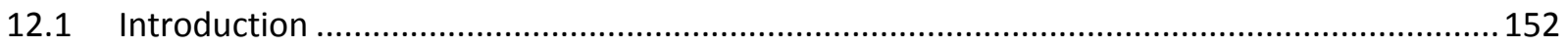

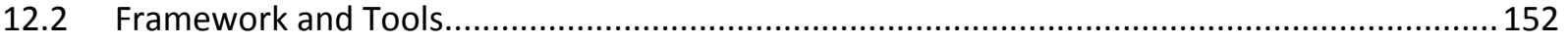

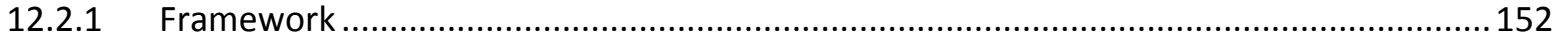

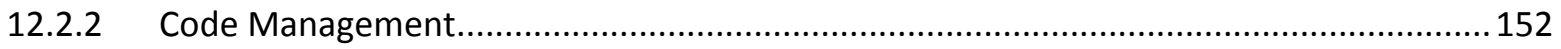




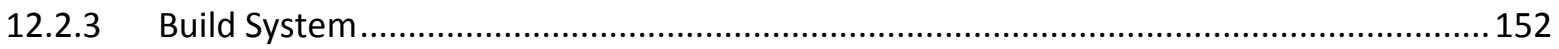

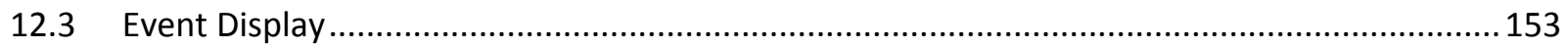

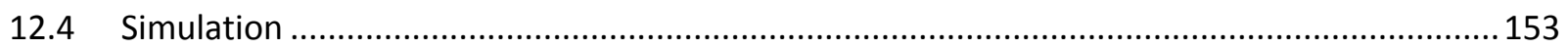

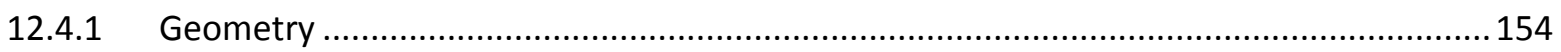

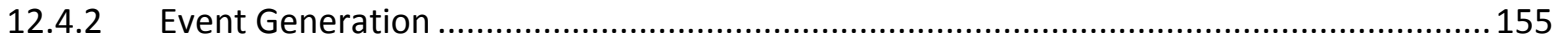

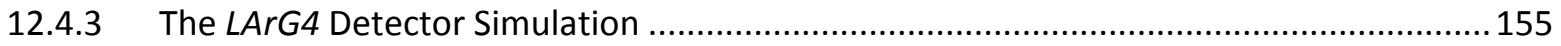

12.4.4 Electron Drift and TPC Electronics Simulation ............................................................ 158

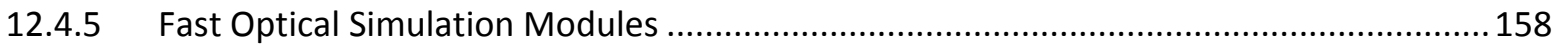

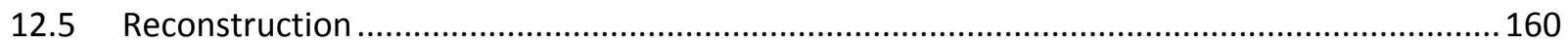

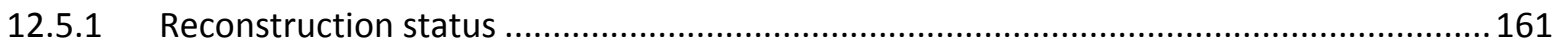

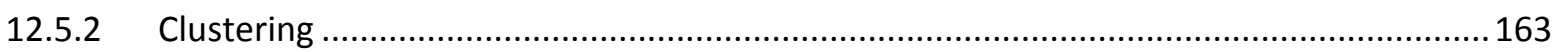

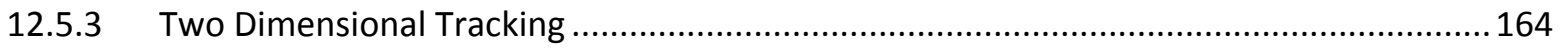

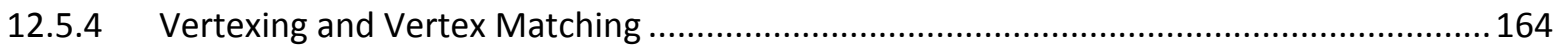

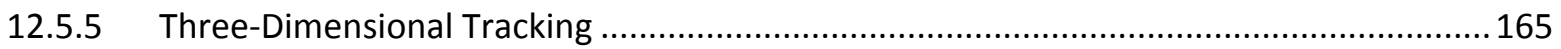

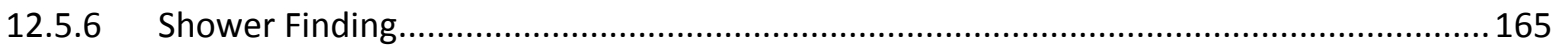

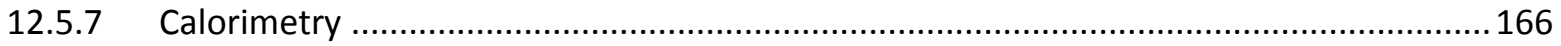

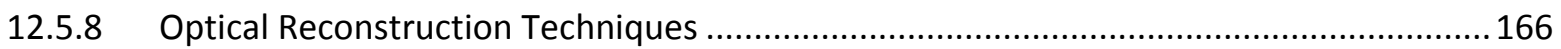

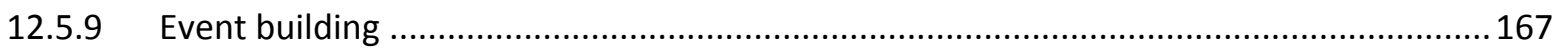

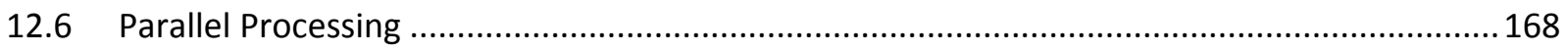

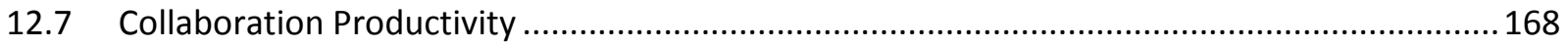

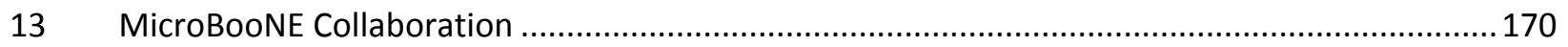

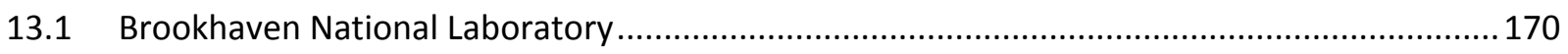

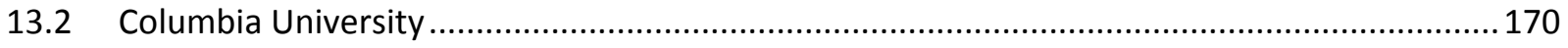

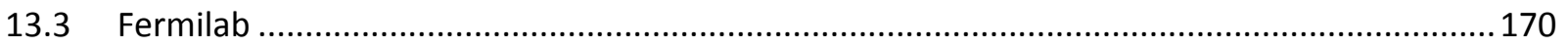

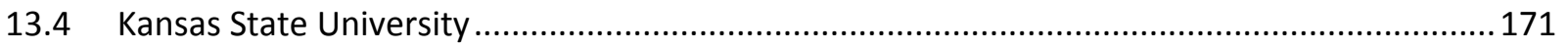

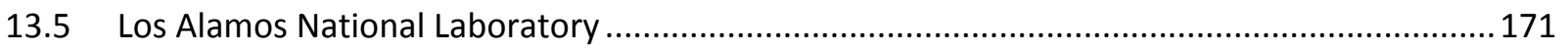

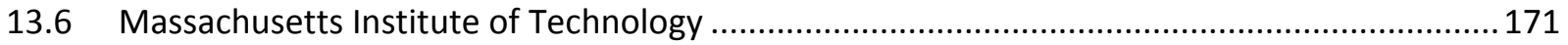

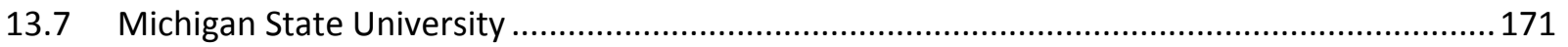

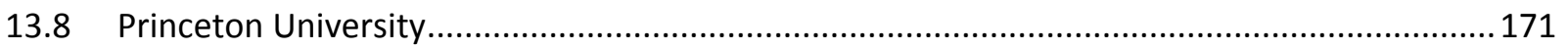

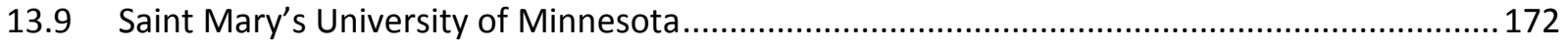

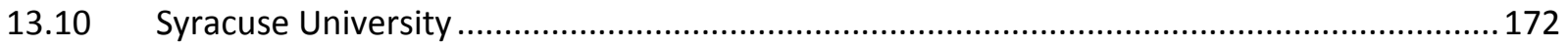

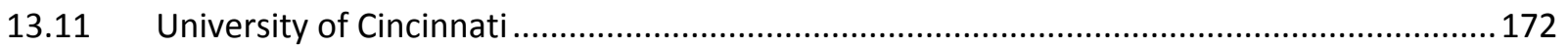




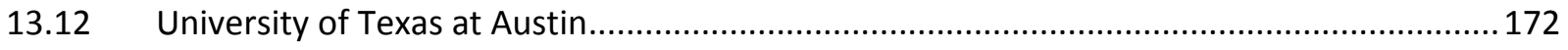

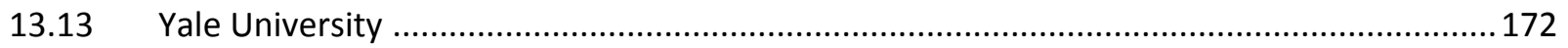




\section{Executive Summary}

MicroBooNE will build, operate, and extract physics from the first large liquid argon time projection chamber (LArTPC) that will be exposed to a high intensity neutrino beam. With its unparalleled capabilities in tracking, vertexing, calorimetry, and particle identification, all with full electronic readout, MicroBooNE represents a major advance in detector technology for neutrino physics in the energy regime of most importance for elucidating oscillation phenomena.

The unique electron-photon discrimination power offered by the LArTPC will allow MicroBooNE to either confirm or rule out the low energy excess of electron-like interactions observed by MiniBooNE, and, if confirmation occurs, to test many models that have developed to explain this excess. The MicroBooNE LArTPC will be capable of observing recoil nucleons produced in the quasi-elastic and resonant processes that dominate low energy neutrino interactions. This ability may help resolve surprisingly large experimental discrepancies $(\sim 30 \%)$ that exist for cross section measurements of such basic processes as $v_{\mu} n \rightarrow \mu^{-} p$ quasi-elastic scattering; and it should allow more detailed tests of models for low energy neutrino interactions that are critical for current and next-generation neutrino oscillation experiments.

Finally, MicroBooNE will fully test the LArTPC technology-including cryostat and TPC design, liquid argon purity, cold electronics, light detection, experiment operations, and, crucially and much neglected, reconstruction software - at the scope and scale that should allow physicists to make the best decisions on the design and operation of very large LArTPC detectors for LBNE and other next-generation oscillation experiments. For all of these reasons, LArTPC development has been widely endorsed as a crucial next-step for the high energy community by $\mathrm{NuSAG}^{1}$ and the P5 panel$^{2}$. MicroBooNE is the next key piece in the US based Integrated Plan for Liquid Argon TPC development ${ }^{3}$.

MicroBooNE received Stage 1 approval by the director of Fermilab, based on the advice of the Program Advisory Committee, in June 2008. The DOE issued a CD-0 mission need statement in September 2009, granted CD-1 approval in March of 2010, and gave CD-2 and CD-3a approval in September 2011. This technical design report (TDR) describes a detector that will achieve the scientific and technological goals of the experiment, and thereby contributes to the DOE critical decisions process.

In brief, the MicroBooNE LArTPC will receive an exposure from the existing Fermilab Booster Neutrino Beam $(\mathrm{BNB})^{4}$ corresponding to $6.6 \times 10^{20}$ protons on target. It will also record interactions from an off axis component of the NUMI neutrino beam. The BNB will be operated in the same configuration as that used during MiniBooNE neutrino operation, thereby significantly reducing the systematic uncertainty in the comparison of MicroBooNE data with that from MiniBooNE. 
The MicroBooNE detector will be located just upstream of the MiniBooNE detector hall in a new Fermilab enclosure, the Liquid Argon Detector Test Facility (LArTF). MicroBooNE employs a $60 \mathrm{t}$ fiducial mass (170 $\mathrm{t}$ total mass) LArTPC contained within a conventional cryostat. The high purity liquid argon in the LArTPC serves as the neutrino target and tracking medium for the particles produced in neutrino interactions. The MicroBooNE design allows for tests of liquid argon purity that can be obtained both with and without use of vacuum. The TPC active volume forms a rectangular solid of dimensions $2.3 \mathrm{~m} \times 2.6 \mathrm{~m} \times 10.4 \mathrm{~m}$.

MicroBooNE detects neutrino interactions through the observation of outgoing charged particles that ionize in, and neutral particles that convert in the liquid argon. Ionization electrons produced by the passage of these charged particles through the liquid argon drift at constant velocity though a uniform electric field to three wire planes on the beam right side of the detector. Electrostatic potentials of the three wire planes allow electrons pass through the first two induction planes to the third collection plane.

Charged particle trajectory reconstruction derives from the known wire positions and the arrival times of electron signals on the wires combined with the time the interaction took place in the detector within the $1.6 \mu \mathrm{s}$ BNB beam spill. The $3 \mathrm{~mm}$ wire pitch enables sub-millimeter position resolutions. The amplitude of the ionization electron signals measures the energy loss of the particles, which, with range information, allows for determination of momentum and particle type.

A photomultiplier tube (PMT) array inside the cryostat provides further precise timing information and allows MicroBooNE to extend its physics measurements to a category of events that contain only very short charged particle tracks in the LArTPC, such as would be produced by $v_{\mu} \mathrm{p} \rightarrow v_{\mu} \mathrm{p}$ elastic scattering.

Induced and direct electrical signals on each TPC wire get amplified and sampled at $2 \mathrm{MHz}$ by an analog to digital converter; signals from the PMT are handled in a similar way. The data acquisition system can operate in a trigger-less mode for both in-spill neutrino interactions and possible non-beam related phenomena, such as supernova neutrinos; and it can be triggered if desired for in-spill neutrino interactions. MicroBooNE forms a core member of the LArSoft software development consortium that seeks to develop common reconstruction software for a range of LArTPC projects at Fermilab and elsewhere.

A strong collaboration of physicists and engineers from three national laboratories and a set of universities that collectively possesses decades of experience in neutrino physics and liquid argon technology tackles the many technical and scientific challenges of MicroBooNE. Description of the work of this collaboration follows in this TDR. Chapter 2 lays out the scientific and technological goals of the experiment in more detail. Chapter 3 tabulates MicroBooNE performance parameters. Project organization and management plans follow in Chapters 4. Chapters 5-11 provide sub-system details at WBS level 2. Chapter 12 summarizes 
the state of MicroBooNE offline software. Chapter 13 lists members and institutions comprising the MicroBooNE collaboration.

\footnotetext{
1 " Recommendations to the Department of Energy and the National Science Foundation on a Future U.S. Program in Neutrino Oscillations", submitted by the Neutrino Scientific Assessment Group. http://www.science.doe.gov/hep/hepap reports.shtm .

${ }^{2}$ Report of the Particle Physics Project Prioritization Panel, June, 2008. http://www.er.doe.gov/hep/hepap reports.shtm .

3 "Integrated Plan for LArTPC neutrino detectors in the US", B. Baller, F. Cavanna, B. Fleming, O. Palamara, R. Plunkett, S. Pordes, G. Rameika, B. Rebel. Reviewed in a Fermilab Director's review, November, 2009. Submitted to DOE in December, 2009.

${ }^{4}$ A. A. Aguilar-Arevalo, et al., [MiniBooNE Collaboration], "The Neutrino Flux prediction at MiniBooNE," Phys. Rev. D 79, 072002 (2009) [arXiv:0806.1449 [hep-ex]].
} 


\section{Scientific Objectives}

\subsection{Overview}

MicroBooNE will be the first large liquid argon time projection chamber (LArTPC) to acquire a high statistics sample of neutrino interactions. With its unparalleled capabilities in tracking, vertexing, calorimetry, and particle identification with full electronic readout, MicroBooNE represents a major advance in detector technology for neutrino physics in the energy regime of most importance for elucidating oscillation phenomena. These capabilities lead to objectives that combine immediate scientific interests with long-term technological innovations.

The experiment has two primary scientific objectives: 1) to resolve the source of the MiniBooNE low energy excess, and 2) to measure a suite of neutrino cross sections. The third important objective of MicroBooNE lies in the area of detector development. MicroBooNE will provide enormous technical experience in the construction, operation, and analysis of a large LArTPC. This experience will directly inform the design of very large LArTPC detectors envisioned for the Long Baseline Neutrino Experiment (LBNE) and beyond. The MicroBooNE design reported in the following chapters balances detector R\&D objectives with the needs for timely extractions of high quality physics.

MicroBooNE's primary scientific and technological goals focus on beam-based applications, but the experiment also provides information on using the LArTPC technology for non-accelerator physics studies. MicroBooNE's relatively small size and surface location prove sufficient for its primary goals but limit its sensitivity to rare, non-beam-related physics signatures. However, at least two intriguing possibilities exist: supernova neutrino observation and the measurement of cosmogenic kaon backgrounds for proton decay searches. The collaboration has identified these as valuable secondary scientific and technological goals.

The scientific goals of MicroBooNE, as well as those of future multi-kiloton $v_{\mu} \rightarrow v_{\mathrm{e}}$ oscillation experiments, rely on the excellent capabilities of LArTPC technology. These notably feature electron/photon differentiation and efficient detection of low-energy protons from the hadronic recoil system in neutrino interactions. The only competing technologies with similar or better particle identification and spatial resolution capabilities are bubble chambers, straw tube trackers, streamer chambers, and emulsion detectors; but these all suffer from serious technological drawbacks. Bubble chambers and streamer chambers operate at inherently low repetition rates, and they, along with emulsion detectors, require scanning systems that lead to considerable delays in data processing. Detectors based on straw tube trackers cannot be feasibly built with sufficient mass for Booster Neutrino Beam energies and suffer from high inhomogeneity. Furthermore, none of these technologies easily scale up to the sizes required for next-generation neutrino oscillation experiments. On the other hand, LArTPCs operate at high 
rate with full electronic readout and processing by the data acquisition system. As a result, LArTPC technology constitutes the only viable option for achieving the MicroBooNE goals in a timely fashion at the level discussed in the sections below.

To meet its objectives MicroBooNE must also develop a new collection of reconstruction software to handle the orders of magnitudes more neutrino interactions that will be recorded compared to previous LArTPC experiments. The development and validation of this software with real physics analyses represents a critical R\&D milestone for future very large LArTPC ${ }^{1}$.

\subsection{Scientific Goals and Related Technical Goals}

This section describes more fully the two primary scientific goals that drive the MicroBooNE design: resolving the source of the MiniBooNE low energy excess and measuring neutrino cross sections on argon. This section also discusses the potential for supernova neutrino detection, a secondary scientific goal and design driver.

\subsubsection{The MiniBooNE Low Energy Excess}

The MiniBooNE experiment performed a short baseline search for the $\nu_{\mu} \rightarrow v_{\mathrm{e}}$ oscillation signature suggested by LSND ${ }^{2}$. MiniBooNE did not observe LSND-like neutrino oscillations in its neutrino data sample, but an unexpected $>3 \sigma$ (statistical and systematic, combined) excess of neutrino interactions producing final state electrons or photons was observed at lower energies. This result generated substantial theoretical interest. Proposed explanations for the source of these events predict either a single electron track or a single photon produced at the neutrino interaction vertex. MiniBooNE cannot discriminate between these two possibilities, as Cerenkov detectors cannot distinguish an electron from a photon converting near the interaction point. Figure 2.1 shows the MiniBooNE low-energy excess above a stacked plot of background sources that arise from both events producing electrons and events producing photons.

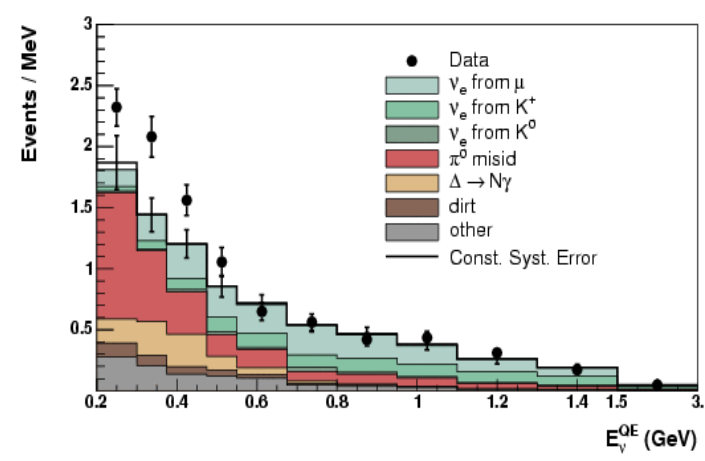

Figure 2.1 MiniBooNE low energy excess in neutrino mode. Backgrounds are shown in the stacked histogram and come from $v_{\mathrm{e}}$ events, which are electron-like, and also misidentified $v_{\mu}$ events, which are photon-like

If in fact the anomalous MiniBooNE events signal true electrons from $v_{\mathrm{e}}$ charged current interactions, explanations from theorists suggest a beyond the Standard Model interpretation. One of the most interesting of these proposes that instead of MiniBooNE observing electron 
neutrino appearance, the low energy excess results from intrinsic electron neutrinos from the beam that disappear at higher energies ${ }^{3}$. This interpretation, combined with recent results from reactor antineutrino experiments ${ }^{4}$, fits well with a model involving oscillations to sterile neutrinos $(3+1$ model) where the null hypothesis is disfavored at $99.93 \%$ CL.

Understanding the source of the MiniBooNE excess thus stands out as the primary physics goal, motivating the following approach:

- MicroBooNE will run in neutrino mode where MiniBooNE observed the excess.

- MicroBooNE will operate in the MiniBooNE neutrino beamline configuration and at nearly the same baseline distance allowing tests of models that predict a dependence of the MiniBooNE excess on the distance from neutrino source to detector.

- MicroBooNE will employ the LArTPC to provide excellent electron/photon discrimination.

- MicroBooNE's data taking run will last long enough to provide sensitivity to a MiniBooNE-type signal at the 3 to $5 \sigma$ level for the predicted excess above background.
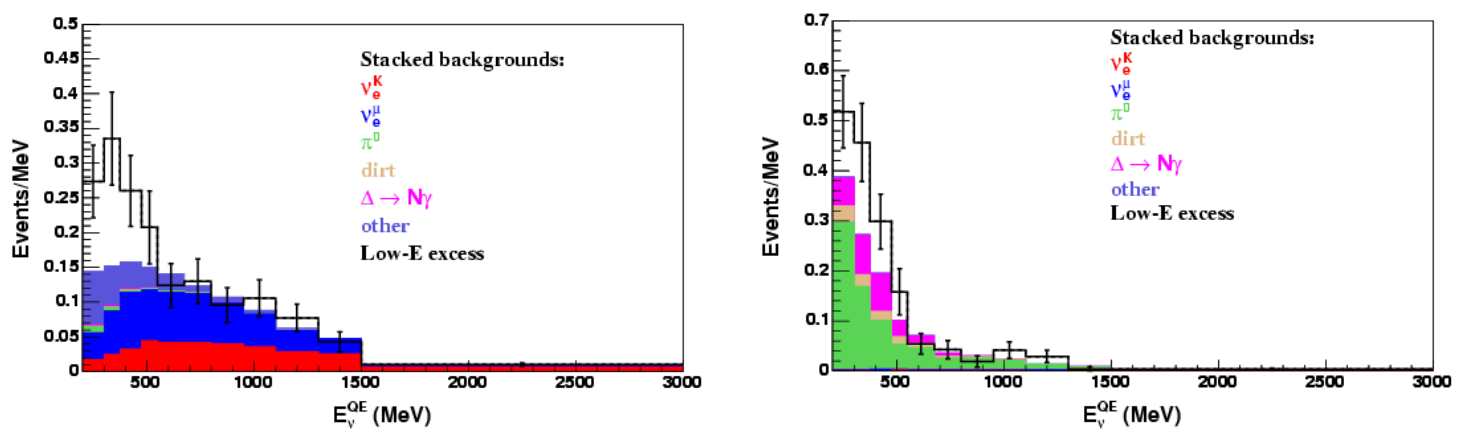

Figure 2.2 MicroBooNE expectations for resolving the MiniBooNE low energy excess. The MicroBooNE analysis is performed under two assumptions: that the low energy excess is due to an electron-like event (left); and that the low energy excess is due to a photon-like event (right). Stacked histograms show the expected backgrounds. Error bars indicate statistical uncertainty. The number of signal events, scaled from MiniBooNE for neutrino flux and fiducial volume, is the same in both plots. Both plots assume $6.6 \times 10^{20}$ POT for the MicroBooNE $60 \mathrm{t}$ fiducial mass.

The MicroBooNE analysis will proceed in two modes, one that assumes that an electron interaction produces the signal (electron-like analysis) and the other that assumes a photon interaction generates the signal (photon-like analysis). Figure 2.2 shows the MicroBooNE expectation for these two analysis modes. Electrons distinguish themselves from photon conversions to an $\mathrm{e}^{+} \mathrm{e}^{-}$pair by through their different apparent ionization rate at the beginning of their trajectory as shown in Figure 2.3. Put simply, an $\mathrm{e}^{+} \mathrm{e}^{-}$pair ionizes at twice the rate of a single electron. 


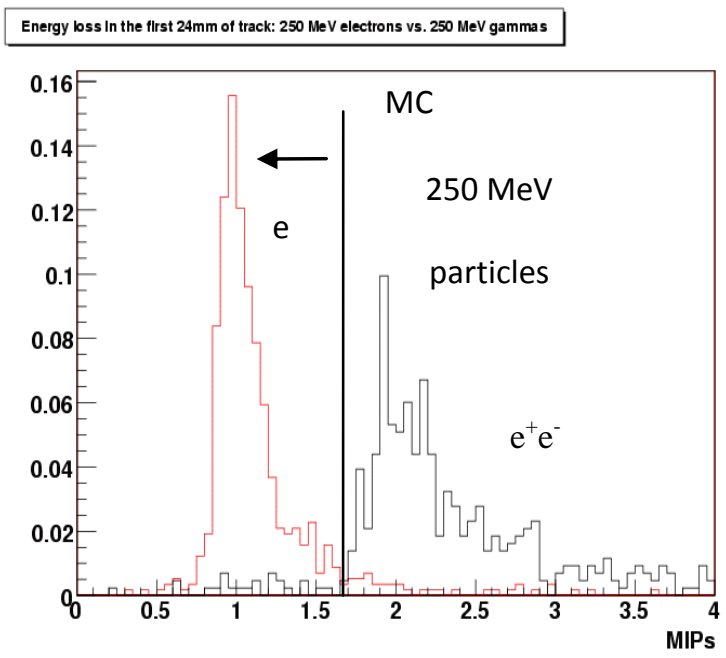

Figure 2.3: Electron vs. photon separation based on a cut on MIP equivalents deposited in the detector.

MicroBooNE expects to observe a $5 \sigma$ or $4 \sigma$ signal if the source arises from electrons or photons, respectively, where the significance estimates assume contributions from both statistical and systematic uncertainties. These sensitivities assume a $\sim 60 \mathrm{t}$ fiducial volume, where the fiducial volume is defined to include events with a primary vertex $35 \mathrm{~cm}$ or more from any surface, or an event outside of this boundary but directed inward so that the final state particles are contained in the TPC.

As can be seen from the MiniBooNE example, photon misidentification backgrounds limit Cerenkov radiation-based detectors. With its much lower level of these backgrounds and considerably higher detection efficiency, the MicroBooNE oscillation sensitivity (Figure 2.4) compares favorably to that of MiniBooNE despite MicroBooNE having five times less detector mass. This illustrates the power of LArTPCs for future $v_{\mu} \rightarrow v_{\mathrm{e}}$ oscillation searches. 


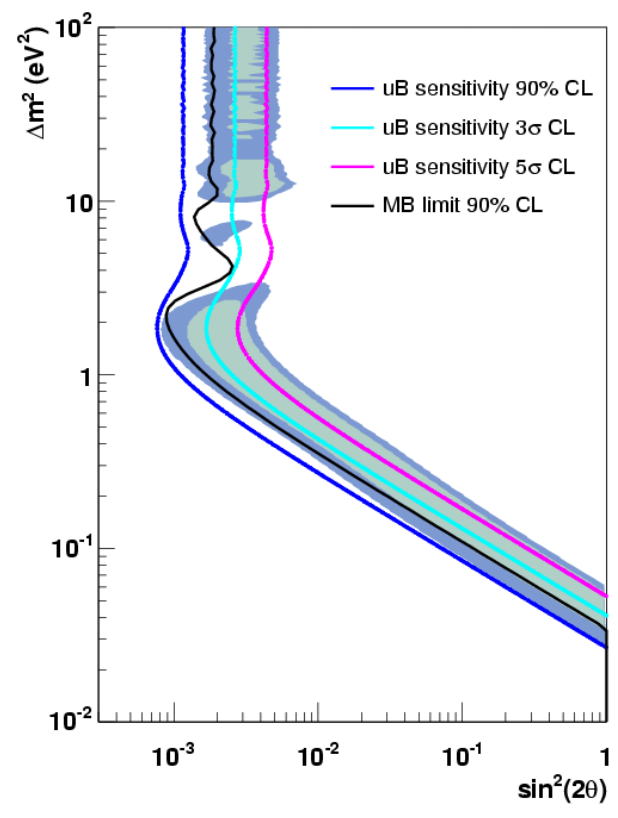

Figure 2.4 Comparison of MiniBooNE (MB) $v_{\mu} \rightarrow v_{\mathrm{e}}$ oscillation result in neutrino mode to MicroBooNE (uB) sensitivity. The sensitivity is comparable because of the improved detection technique, which greatly reduces backgrounds, despite the fact that MicroBooNE is $\sim 5$ times smaller than MiniBooNE,

\subsubsection{Cross Section Measurements}

MicroBooNE proposes to make the first measurement of exclusive final states from neutrino scattering on argon. Table 2.1 lists interaction rates for the $60 \mathrm{t}$ fiducial volume and displays a rich physics program.

Four cross section measurements in particular attract great interest from the neutrino community. These measurements stress the value of:

- Excellent efficiency for triggering on low energy objects, such as final states with a single proton with a kinetic energy above $40 \mathrm{MeV}$;

- Excellent resolution in position and momentum vector determination for protons produced at the target;

- Ability to observe and reconstruct complicated topologies;

- Sufficient statistics to reduce statistical uncertainties to the same order as systematic effect for rare processes.

These four features constitute the design drivers for the MicroBooNE detector that arise from the cross section physics. 


\subsubsection{Elastic neutrino-proton scattering}

MicroBooNE can measure the elastic scattering cross section ratio $\sigma\left(v_{\mu} p \rightarrow v_{\mu} p\right) / \sigma\left(v_{\mu} n \rightarrow \mu^{-} p\right)$. When combined with polarized electron-proton/deuterium cross section measurements, this ratio allows a determination of $\Delta s$, the fraction of the nucleon spin carried by strange quarks ${ }^{5}$. Measuring this ratio at low values of $\mathrm{Q}^{2}$ gives MicroBooNE the potential to improve the determination of the value of $\Delta s$ by almost an order of magnitude in global fits of strange form factors ${ }^{6}$. Dark matter searches require better knowledge of $\Delta s$, with the current uncertainty on this quantity limiting the sensitivity of many such searches.

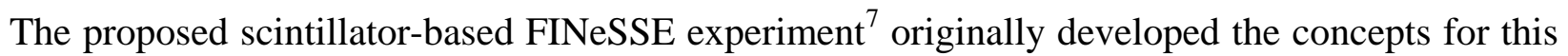
$\Delta s$ measurement. MicroBooNE improves upon FINeSSE in that recoil protons remain wellcontained in the TPC volume where they can be readily identified by $\mathrm{dE} / \mathrm{dx}$ and energy-analyzed via their range range in the LAr. Triggering on events containing a single recoil proton with at least $40 \mathrm{MeV}$ kinetic energy protons for this measurement constitutes a design driver for the MicroBooNE PMT system.

\begin{tabular}{lr} 
production mode & \# events \\
\hline CC QE $\left(\nu_{\mu} n \rightarrow \mu^{-} p\right)$ & 60,161 \\
NC elastic $\left(\nu_{\mu} N \rightarrow \nu_{\mu} N\right)$ & 19,409 \\
CC resonant $\pi^{+}\left(\nu_{\mu} N \rightarrow \mu^{-} N \pi^{+}\right)$ & 25,149 \\
CC resonant $\pi^{0}\left(\nu_{\mu} n \rightarrow \mu^{-} p \pi^{0}\right)$ & 6,994 \\
NC resonant $\pi^{0}\left(\nu_{\mu} N \rightarrow \nu_{\mu} N \pi^{0}\right)$ & 7,388 \\
NC resonant $\pi^{ \pm}\left(\nu_{\mu} N \rightarrow \nu_{\mu} N^{\prime} \pi^{ \pm}\right)$ & 4,796 \\
CC DIS $\left(\nu_{\mu} N \rightarrow \mu^{-} X, W>2 \mathrm{GeV}\right)$ & 1,229 \\
NC DIS $\left(\nu_{\mu} N \rightarrow \nu_{\mu} X, W>2 \mathrm{GeV}\right)$ & 456 \\
NC coherent $\pi^{0}\left(\nu_{\mu} A \rightarrow \nu_{\mu} A \pi^{0}\right)$ & 1,694 \\
CC coherent $\pi^{+}\left(\nu_{\mu} A \rightarrow \mu^{-} A \pi^{+}\right)$ & 2,626 \\
NC kaon $\left(\nu_{\mu} N \rightarrow \nu_{\mu} K X\right)$ & 39 \\
CC kaon $\left(\nu_{\mu} N \rightarrow \mu^{-} K X\right)$ & 117 \\
other $\nu_{\mu}$ & 3,678 \\
\hline total $\nu_{\mu} \mathrm{CC}$ & 98,849 \\
total $\nu_{\mu}$ NC $+\mathrm{CC}$ & 133,580 \\
\hline \hline$\nu_{e}$ QE & 326 \\
$\nu_{e} \mathrm{CC}$ & 657
\end{tabular}

Table 2.1: Expected event rates in MicroBooNE for the TDR design.

\subsubsection{Coherent pion production}

Coherent pion production in neutral current (NC) and charged current (CC) events provides a second set of cross section measurement that highlight the value of high resolution and clean reconstruction of nucleons in MicroBooNE. A K2K analysis suggests that very little coherent 
$\mathrm{CC} \pi^{+}$production $^{8}$, a result confirmed by SciBooNE measurements ${ }^{9}$. From a theoretical point of view this observation does not harmonize with the larger coherent $\mathrm{NC} \pi^{0}$ signal reported by MiniBooNE $^{10}$. Two features characterize coherent neutrino-nucleus interactions, a sharply forward-scattered final state lepton and pion, and the absence of debris from nuclear breakup. MicroBooNE provides both excellent forward angle resolution and high sensitivity for observation of protons and de-excitation photons produced in non-coherent processes.

\subsubsection{Kaon production in low energy neutrino scattering}

Measurement of kaon production by neutrinos addresses two important issues. First, a study of these events should prove to be directly relevant to future proton decay measurements by multikton LArTPCs in the $\mathrm{p} \rightarrow \mathrm{K}^{+} v$ decay channel. Kaons produced in MicroBooNE lie in the same energy range as those that would be produced via $\mathrm{p} \rightarrow \mathrm{K}^{+} v$. Examining these kaons in MicroBooNE will thus improve the background estimates for future proton decay experiments in this mode. Second, kaon production provides a nice test of reconstructing complicated topologies (the $\mathrm{K} \rightarrow \pi \rightarrow \mu \rightarrow \mathrm{e}$ decay chain), a design-driver for the experiment.

\subsubsection{Photon production in low energy neutrino scattering}

MicroBooNE could demonstrate that photons and not electrons produce the MiniBooNE low energy excess. In this case, one could then conclude that $v_{\mathrm{e}}$ production is well understood at low energies where the flux is dominated by muon decay. This would allow MicroBooNE to make the first measurement of the $v_{\mathrm{e}}$ cross section in the $0.1-1.0 \mathrm{GeV}$ energy range, an important measurement for precision long-baseline neutrino oscillation studies.

\subsubsection{Supernova Detection and Proton Decay Background Measurements}

MicroBooNE' surface location limits its palette of non-accelerator neutrino physics topics. This siting reflects a conscious design decision to focus on beam related physics, and not to expand the design in a substantial way to incorporate non-accelerator physics.

One exciting non-accelerator physics topic open to the experiment with only modest expansion to its design is supernova ( $\mathrm{SN}$ ) detection through a short pulse of neutrino interactions observed in the detector. If a supernova were to occur in our galaxy, MicroBooNE would detect more events than were observed, in total, from SN 1987a. Until SNO+ or NOvA turn on, MicroBooNE and MiniBooNE represent the only neutrino detectors in North America capable of obtaining a significant sample of such events. Observations from these detectors would complement those from Japan (Super-Kamiokande and KamLAND) and from Europe (Borexino and ICARUS). Moreover, because these SN events produce electrons in the $>10 \mathrm{MeV}$ range, the design requirements on MicroBooNE do not substantially extend beyond those already in place for collecting a substantial Michel muon decay electron sample for energy calibration studies. In the process of seeking this physics, MicroBooNE addresses several technical issues for future detectors. 

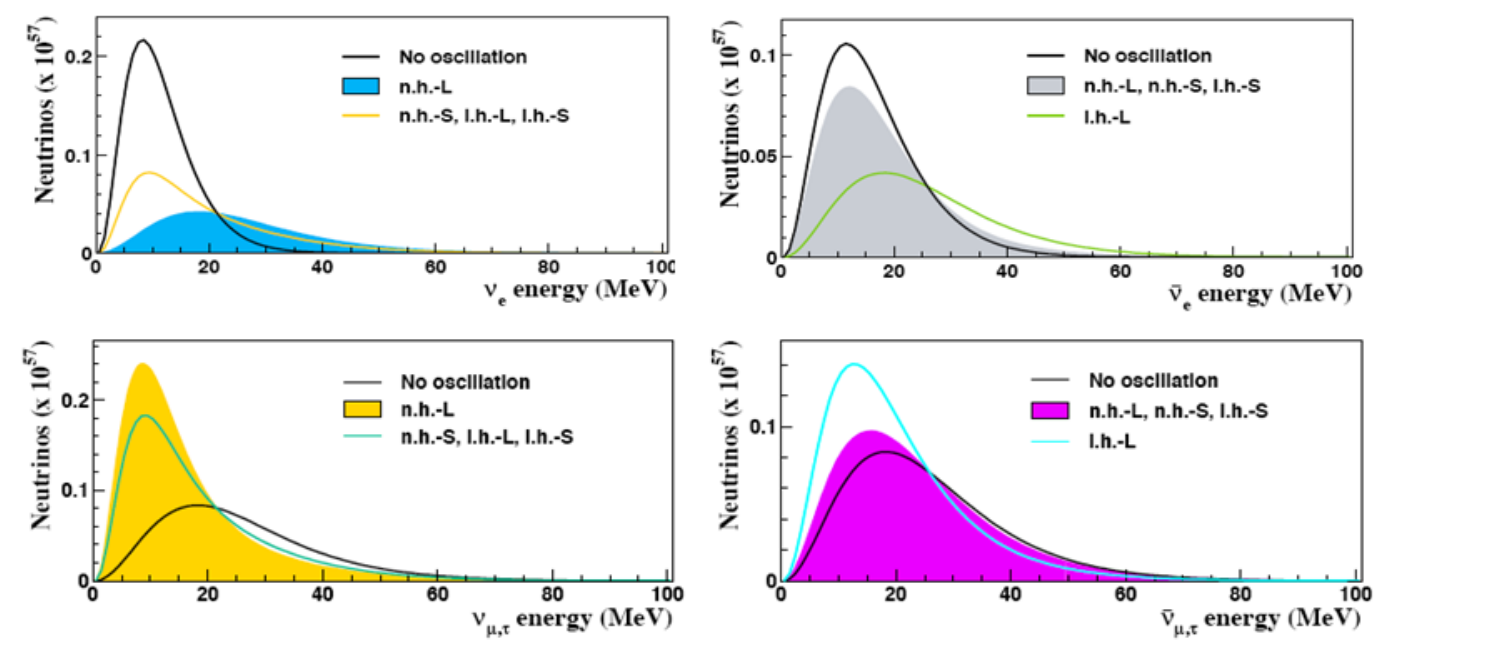

Figure 2.5 Energy distributions for neutrinos produced by supernovae in a 3 kiloton LArTPC ${ }^{11}$. The solid black line shows the expectation before oscillations. Neutrino oscillations affect the fluxes considerably. The filled histogram shows the flux expectation if neutrinos have normal hierarchy ("n.h.") and large $\sin ^{2} \theta_{13}$ ("L") $\sim 3 \times 10^{-4}$. The cyan line shows alternative oscillation scenarios (see reference for details).

Figure 2.5 shows the expected neutrino spectrum for neutrinos produced in a supernova and detected in a large (3 kiloton) LArTPC. Neutrino oscillations dramatically affect the energy spectrum of these neutrinos. The exact energy range where these effects become visible depends on the neutrino mass hierarchy and the value of neutrino mixing angle $\theta_{13}$. MicroBooNE would likely be insensitive to the details of these oscillation effects once one convolves the energy dependence of the cross section with the fluxes; and using SN neutrinos to probe the mass hierarchy and $\theta_{13}$ thus requires a larger experiment. Nevertheless, SN rates in MicroBooNE could be significant, with potentially twice the number of events observed compared to SuperKamiokande for SN 1987a. Charged current $v_{\mathrm{e}}-\mathrm{Ar}$ events would comprise the most important sample, as the incident neutrino energy can be directly related to the outgoing electron energy. The expected set of $25-30$ neutrino interactions can be used to study the energy spectrum of the SN neutrinos in conjunction with other detectors throughout the world..

An additional non-accelerator based physics goal of MicroBooNE consists of measuring cosmic ray backgrounds to proton decay searches in the $\mathrm{p} \rightarrow \mathrm{K}^{+} v$ mode suggested by supersymmetry. While next generation multi-kiloton LArTPC detectors will achieve sensitivities well beyond current limits from Super-Kamiokande in this decay mode, they may become limited by spurious signals associated with cosmogenic kaon production. These backgrounds become more severe as the detector overburden decreases. Sited on the surface, and with excellent particle identification capabilities, MicroBooNE will be able to directly measure cosmic ray backgrounds for $\mathrm{p} \rightarrow \mathrm{K}^{+} v$ and other proton decay modes that can be extrapolated to experiments operating at greater depths. 


\subsection{Development Goals}

As described in Chapter 1, a LArTPC presents an attractive detector concept for a long baseline neutrino experiment of mass 20 kilotons or more, referred to henceforth as LAr20. Several challenges must be addressed before a sufficiently low-risk conceptual design can be developed. These include:

1. Achieving and maintaining adequate argon purity in a large non-evacuable cryostat;

2. Developing and operating high channel-count $\left(\sim 10^{6}\right)$ low-noise electronics at liquid cryogen temperatures;

3. Developing simulation and reconstruction software tools leading to physics analyses based on a large sample of neutrino interactions;

4. Understanding the construction cost of such a large detector;

5. Mitigating the oxygen deficiency hazard (ODH) in an underground facility.

MicroBooNE will significantly improve our understanding of the first three issues with the development goals listed below. The fourth issue must be addressed by engineered controls that are unique to a deep underground facility. The design standards and methods for ODH hazard mitigation in MicroBooNE have existed at Fermilab for many years.

MicroBooNE represents a major step beyond the largest LArTPC that has operated in the US, ArgoNeuT, small test experiment with 480 channels of electronics that collected neutrino interaction data in the NuMI beam at Fermilab over a six-month period in 2009-2010. Experience gained on ArgoNeuT has contributed greatly to MicroBooNE, and significant overlap in the collaboration membership exists between these two experiments. Other liquid argon development facilities at Fermilab that support MicroBooNE and future LArTPC detectors include:

- The PAB materials test stand for measuring the contamination of materials in liquid argon and low temperature gaseous argon;

- A LArTPC cosmic ray test stand for testing electronics;

- The Liquid Argon Purity Demonstration (LAPD) project, for measuring purity in a large non-evacuated vessel (but without benefit of a functioning TPC);

- Prototyping work for the LBNE experiment.

MicroBooNE development goals were established under the restriction that they not compromise the scientific goals of the experiment. They were chosen to provide useful information for the future and also to ensure that the detector will function as proposed. The risk of damage to the 
detector during development activities should be minimal. Finally, MicroBooNE activities should provide timely information to guide the design of LAr20.

\subsubsection{Argon Purity}

Detector components internal to the MicroBooNE cryostat are fabricated, cleaned, and installed using methods that are readily adaptable to LAr20. The MicroBooNE cryostat is not intended to be a prototype of this detector in that its design allows vacuum pumping to remove contaminants such as water and oxygen. However, initial filling of the cryostat will be performed without vacuum pumping. At this point in its operation, MicroBooNE will determine whether an oxygen equivalent impurity level of 100 parts-per-trillion (ppt) can be achieved without resorting to vacuum procedures. Careful documentation of the detector construction will benefit the community in the design of larger LAr detectors if this test is successful.

\subsubsection{Large Scale Cryogenic Low-noise Electronics}

The MicroBooNE experiment will use CMOS pre-amplifiers of the same design as those anticipated for use in LAr20 and with operation in the LAr. Developing and operating this electronics with MicroBooNE comprises a major development goal for LAr20 and beyond.

\subsubsection{MicroBooNE Software Development}

MicroBooNE and its data set will serve to help develop a fully automated event reconstruction and simulation package for neutrino interactions and proton decay measurements in a LArTPC. No fully automated event reconstruction software of this type exists at this time.

\subsubsection{Construction Cost}

Two detector concepts remain under consideration for LBNE, a water Cherenkov detector and LAr20. The combination of signal efficiency and background rejection make a LArTPC six times more sensitive to long baseline neutrino physics than a water Cherenkov detector of the same mass. Equivalently, a liquid argon detector may be six times smaller than a water Cherenkov detector for the same physics sensitivity. Reliable data on the construction cost of a medium scale LAr detector such as MicroBooNE will help refine the cost-benefit relationship of LAr20 to the water Cherenkov detector. The MicroBooNE cost information will be applicable for all elements of LAr20 with the exception of the cryostat and the detector facility.

\footnotetext{
1 "Recommendations to the Department of Energy and the National Science Foundation on a Future U.S. Program in Neutrino Oscillations", submitted by the Neutrino Scientific Assessment Group.

2 "Evidence for anti-muon neutrino -> anti-electron neutrino oscillations from the LSND experiment at LAMPF", Phys. Rev. Lett. 77:3082-3085, 1996.

3 "Short Baseline $v_{\mu}$ bar-> vebar Oscillations", Phys. Rev. D82:093016, 2010.

4 "The Reactor Anti-Neutrino Anomaly", [arXiv:1101.2755[hep-ex]].

${ }^{5}$ S.F. Pate, D.W. McKee and V. Papavassiliou, "Strange Quark Contribution to the Vector and Axial Form Factors of the Nucleon: Combined Analysis of G0, HAPPEX and Brookhaven E734Data," Phys. Rev. C 78, 015207 (2008) [arXiv:0805.2889 [hep-ex]].
} 
${ }^{6}$ ] J. Schaub, "Global Analysis of the Strangeness Vector and Axial Vector Form Factors of the Nucleon and their Uncertainties," talk presented at the PAVI09 International Workshop "From Parity Violation to Hadronic Structure and more," Bar Harbor, ME (2009); to be published in the proceedings.

${ }^{7}$ L. Bugel et al. [FINeSSE Collaboration], "A proposal for a near detector experiment on the booster neutrino beamline: FINeSSE: Fermilab Intense Neutrino Scattering Scintillator Experiment," [arXiv:hep-ex/0402007].

${ }^{8}$ A. Rodriguez [K2K Collaboration], "Measurement of single charged pion production in the charged-current \%interactions of neutrinos in a $1.3 \mathrm{GeV}$ wide band beam," Phys. Rev. D 78, 032003 (2008) [arXiv:0805.0186 [hep-ex]].

${ }^{9}$ SciBooNE Collaboration, "Search for charged current coherent pion production on carbon in a few-GeV neutrino beam", Phys. Rev. D 78, 112004 (2008).

${ }^{10}$ ] A. A Aguilar-Arevalo, et al., [MiniBooNE Collaboration], "First Observation of Coherent $\pi^{\circ}$ Production in Neutrino Nucleus Interactions with $\mathrm{E}_{\mathrm{v}}<\$ 2 \mathrm{GeV}$," Phys. Lett. B 664, 41 (2008) [arXiv:0803.3423 [hep-ex]].

${ }^{11}$ A. Bueno, I. Gil Botella and A. Rubbia, "Supernova neutrino detection in a liquid argon TPC," arXiv:hepph/0307222; I. Gil Botella and A. Rubbia, "Oscillation effects on supernova neutrino rates and spectra and detection of the shock breakout in a liquid argon TPC," JCAP 0310, 009 (2003) [arXiv:hep-ph/0307244]. 


\section{Design Criteria and Parameter Tables}

This chapter provides tabular summaries of the design and performance requirements on the MicroBooNE detector and experiment needed to meet its scientific goals, and of the technical choices made in order to satisfy these requirements.

\subsection{Physics Requirements}

MicroBooNE's two primary physics goals focus on identifying the source of low energy electron-neutrino-like interactions observed by MiniBooNE, and measuring cross sections for $v_{\mu}$ Ar scattering. Secondary goals include a search for nearby supernova neutrino events and a study of cosmic ray kaon production backgrounds to proton decay searches. Chapter 2 provides more details. The table below summarizes requirements for the Booster Neutrino Beam and MicroBooNE detector to accomplish these goals.

\begin{tabular}{|c|c|}
\hline \multicolumn{2}{|c|}{ Physics Requirements } \\
\hline Measurement & Requirements \\
\hline $\begin{array}{l}\text { MiniBooNE low energy excess established } \\
\text { as electrons to }>5 \sigma\end{array}$ & $6.6 \times 10^{20} \mathrm{POT}, 70 \mathrm{t}$ active volume. \\
\hline $\begin{array}{l}\text { MiniBooNE low energy excess established } \\
\text { as photons to }>4 \sigma\end{array}$ & $6.6 \times 10^{20} \mathrm{POT}, 70 \mathrm{t}$ active volume. \\
\hline $\begin{array}{l}\text { Electron/photon discrimination by track } \\
\mathrm{dE} / \mathrm{dx} \text { near vertex. }\end{array}$ & $\begin{array}{l}3 \mathrm{~mm} \text { pitch, } 3 \mathrm{~mm} \text { plane separation, and } 80 \% \text { efficiency } \\
\text { for e/ } \gamma \text { separation algorithm. }\end{array}$ \\
\hline $\begin{array}{l}v_{\mu} \rightarrow v_{e} \text { search above } 475 \mathrm{MeV} \text { to overlap } \\
\text { LSND } \sin ^{2}(2 \theta) \text { at high } \Delta \mathrm{m}^{2} 90 \% \mathrm{CL} \text { allowed } \\
\text { region to } 3 \sigma .\end{array}$ & $\begin{array}{l}6.6 \times 10^{20} \mathrm{POT}, 70 \mathrm{t} \text { active volume, and MiniBooNE } \\
\text { baseline. }\end{array}$ \\
\hline $\begin{array}{l}\text { Cross section measurements in Table } 2.1 \\
\text { with } \sigma_{\text {STAT }} \sim \sigma_{\text {SYST. }}\end{array}$ & $6.6 \times 10^{20} \mathrm{POT}, 70 \mathrm{t}$ active volume. \\
\hline $\begin{array}{l}\sigma_{\theta} \text { sufficient for coherent pion production } \\
\text { identification. }\end{array}$ & $3 \mathrm{~mm}$ pitch, $3 \mathrm{~mm}$ plane separation. \\
\hline $\begin{array}{l}\text { Elastic proton scattering via recoil proton } \\
\text { detection. }\end{array}$ & $40 \mathrm{MeV}$ equivalent summed PMT trigger threshold. \\
\hline $\begin{array}{l}\gamma / \mathrm{e} / \pi / \mathrm{K} / \mathrm{p} \text { separation over full BNB energy } \\
\text { range and detection of nucleons from } \mathrm{Ar} \\
\text { breakup. }\end{array}$ & $\begin{array}{l}3 \mathrm{~mm} \text { pitch, } 3 \mathrm{~mm} \text { plane separation, and }>50 \mathrm{MIP} \text { single } \\
\text { TPC channel dynamic range. }\end{array}$ \\
\hline Supernova detection via SNEWS trigger. & 1 hour data buffering. \\
\hline
\end{tabular}




\subsection{TPC and PMT Detector System Parameters}

The following table summarizes the set of design parameters for the MicroBooNE time projection chamber (TPC) and light collection system (PMT) that will allow the experiment to make its physics measurements. Chapters 8 and 9 provide details of the TPC and PMT systems, respectively.

\begin{tabular}{|c|c|c|}
\hline \multicolumn{3}{|c|}{ Active Detectors Requirements } \\
\hline Parameter & Value & Motivation \\
\hline TPC Dimensions & $\begin{array}{l}2.325 \mathrm{~m} \text { vertically } \times 2.5604 \mathrm{~m} \\
\text { horizontally } \times 10.368 \mathrm{~m} \text { in the } \\
\text { beam direction as measured } \\
\text { from the edges of the wire } \\
\text { carriers. }\end{array}$ & $\begin{array}{l}\text { The transverse size is determined by } \\
\text { the maximum diameter cryostat that } \\
\text { can be transported to Fermilab by } \\
\text { truck. The length is determined by the } \\
70 \mathrm{t} \text { active mass requirement. }\end{array}$ \\
\hline Wire Pitch & $3 \mathrm{~mm}$ & $\begin{array}{l}\text { Minimum wire pitch possible given the } \\
\text { electronics } \mathrm{S} / \mathrm{N} \text { and LAr purity that } \\
\text { satisfies detector spatial resolution } \\
\text { requirements. }\end{array}$ \\
\hline $\begin{array}{l}\text { Wire Plane } \\
\text { Separation }\end{array}$ & $3 \mathrm{~mm}$ & $\begin{array}{l}\text { Wire plane separation should be } \\
\text { roughly equal to the wire pitch. }\end{array}$ \\
\hline Wire diameter & 150 microns & $\begin{array}{l}\text { Large enough to withstand the tension } \\
\text { put on the wire. }\end{array}$ \\
\hline Wire Tension & $1 \mathrm{~kg}(9.8 \mathrm{~N})$ & $<0.5 \mathrm{~mm}$ sag for a $5 \mathrm{~m}$ long wire. \\
\hline $\begin{array}{l}\text { Wire length } \\
\text { construction } \\
\text { tolerance } \\
\end{array}$ & $\begin{array}{l} \pm 0.02 \% \text { or } \pm 0.25 \mathrm{~mm} \\
\text { whichever is greater }\end{array}$ & $\begin{array}{l}\text { Equivalent to } \pm 10 \% \text { tension variation } \\
\text { at } 1 \mathrm{~kg} \text {, corresponding to } \pm 0.05 \mathrm{~mm} \text { of } \\
\text { sag for } 5 \mathrm{~m} \text { wire. }\end{array}$ \\
\hline Wire composition & $\begin{array}{l}150 \mu \mathrm{m} \text { stainless steel wire, } 2 \\
\mu \mathrm{m} \text { copper plating with gold } \\
\text { flash }\end{array}$ & $\begin{array}{l}\text { Copper plating reduces resistance of } \\
\text { wire. Gold flash provide oxidation } \\
\text { protection. }\end{array}$ \\
\hline $\begin{array}{l}\text { Number of read out } \\
\text { wire planes }\end{array}$ & 3 & $\begin{array}{l}\text { Redundancy needed to reconstruct } \\
\text { tracks traveling along a wire direction } \\
\text { in one plane }\end{array}$ \\
\hline Wire orientation & $\begin{array}{l}1 \text { vertical plane, } 2 \text { planes at } \pm \\
60 \text { degrees from the vertical }\end{array}$ & $\begin{array}{l}\text { Large angle tracks from low energy } \\
\text { neutrino interactions require large } \\
\text { angle differences between wire plane } \\
\text { orientations. }\end{array}$ \\
\hline $\begin{array}{l}\text { Field cage } \\
\text { dimensions and } \\
\text { tubing size }\end{array}$ & $\begin{array}{l}10.5 \times 2.5 \times 2.6 \mathrm{~m} \text { overall. } 1 \mathrm{in} \text {. } \\
\text { diameter tubing. }\end{array}$ & $\begin{array}{l}\text { Overall dimensions driven by TPC size. } \\
\text { Tube size maximizes strength while } \\
\text { minimizing field variations. }\end{array}$ \\
\hline $\begin{array}{l}\text { Photomultiplier } \\
\text { tubes }\end{array}$ & 30 8" diameter PMTs & $\begin{array}{l}\text { Sufficient photocathode coverage to } \\
\text { trigger on } 40 \mathrm{MeV} \text { protons. }\end{array}$ \\
\hline
\end{tabular}




\subsection{TPC High Voltage Requirements}

The high voltage system for a LArTPC entails establishing very high electrostatic potentials in the liquid cryostat. The voltage must remain very stable, and the voltage distribution system must dissipate minimal power. The parameters for this subsystem of the TPC are thus tabulated separately here. More details can be found in Chapter 8 .

\begin{tabular}{|c|c|c|}
\hline \multicolumn{3}{|c|}{ High Voltage Requirements } \\
\hline Parameter & Value & Motivation \\
\hline Cathode Voltage & $128 \mathrm{kV}$ & $\begin{array}{l}500 \mathrm{~V} / \mathrm{cm} \text { nominal operating drift } \\
\text { field. Power supply capable of } \\
150 \mathrm{kV} \text { (or up to } 600 \mathrm{~V} / \mathrm{cm} \text { drift). }\end{array}$ \\
\hline Ripple tolerance & $<1 \times 10^{-5}$ Vpp@1mA load & $\begin{array}{l}\text { Eliminate drift velocity variations } \\
\text { and external noise }\end{array}$ \\
\hline Resistors linking field cage rings & $250 \mathrm{M} \Omega ; 4$ parallel resistors per gap. & $\begin{array}{l}\text { Minimizing power into cryostat } \\
\text { while maintaining redundancy. }\end{array}$ \\
\hline
\end{tabular}

\subsection{Cryogenics and Argon Purification Requirements}

Electrons must maintain constant drift velocity over $2.5 \mathrm{~m}$ drift distances in the cryostat. This drift velocity must be uniform throughout the detector and not vary with time. The table below summarizes requirements on the cryogenic system and cryostat needed to achieve these goals. More details can be found in Chapters 6 and 7.

\begin{tabular}{|l|l|l|}
\hline \multicolumn{2}{|c|}{ Cryogenics \& Purification System Requirements } \\
\hline \multicolumn{1}{|c|}{ Parameter } & \multicolumn{1}{|c|}{ Value } & \multicolumn{1}{c|}{ Motivation } \\
\hline Argon purity & $\begin{array}{l}<100 \mathrm{ppt} \mathrm{O}_{2} \\
<1 \mathrm{ppm} \mathrm{N} 2\end{array}$ & $\begin{array}{l}\text { Need to identify a minimum ionizing } \\
\text { particle at the longest drift distance }\end{array}$ \\
\hline $\begin{array}{l}\text { Liquid argon receiving } \\
\text { rate }\end{array}$ & One tank truck per day & $\begin{array}{l}\text { Provides time for LAr purity validation } \\
\text { in tank truck before cryostat fill }\end{array}$ \\
\hline $\begin{array}{l}\text { Cryostat LAr } \\
\text { temperature range }\end{array}$ & $<1^{\circ} \mathrm{K}$ & $\begin{array}{l}\text { Eliminate the effect of convection } \\
\text { currents on the electron drift }\end{array}$ \\
\hline Pressure stability & $<2$ psi during operation & Temperature control. \\
\hline Liquid argon flow rate & 1 volume change/day & $\begin{array}{l}\text { Ensure that adequate purity can be } \\
\text { achieved after filling the cryostat }\end{array}$ \\
\hline $\begin{array}{l}\text { UPS backup control } \\
\text { power }\end{array}$ & $>24$ hours & $\begin{array}{l}\text { Maintains LAr purity and cryostat } \\
\text { controls while gas generators come } \\
\text { online. }\end{array}$ \\
\hline Cryostat head removal & $\begin{array}{l}\text { Head removable and } \\
\text { replaceable at least once. }\end{array}$ & $\begin{array}{l}\text { Allow for repair or replacement of } \\
\text { detector components. }\end{array}$ \\
\hline Cryostat insulation & $<15 \mathrm{~W} / \mathrm{m}^{2}$ & $\begin{array}{l}\text { Eliminate the effect of convection } \\
\text { currents on the electron drift }\end{array}$ \\
\hline LAr bubble formation & No observable rate. & $\begin{array}{l}\text { Eliminate the effect of convection } \\
\text { currents on the electron drift }\end{array}$ \\
\hline Cryostat MAWP & 30 psig/full vacuum & $\begin{array}{l}\text { Allows use of reasonably-sized code- } \\
\text { stamped relief valves. }\end{array}$ \\
\hline Seismic load & UBC Zone 1, <0.1g acceleration & Maximum seismic event at Fermilab. \\
\hline
\end{tabular}




\subsection{Electronics and DAQ Requirements}

The TPC readout electronics must be capable of measuring ionization signals produced by objects ranging from a minimum ionizing particle (MIP) to a very slow proton from an argon nuclear breakup to allow particle identification by $\mathrm{dE} / \mathrm{dx}$. Electron drift times must be determined to $\sim 1 \mu$ s precision over a range of $1.6 \mathrm{~ms}$ to ensure high spatial resolution near the neutrino interaction vertex, the region of most interest for electron-photon discrimination and exclusive reaction channel identification. The PMT readout must be sufficient linearity to allow the formation of a $40 \mathrm{MeV}$ energy trigger on a single recoil proton, and must provide sufficient timing precision to allow the PMT to be used in coincidence triggers. The data acquisition system (DAQ) must be able to record data from the TPC in an untriggered mode and to buffer the data for up to an hour to allow for supernova detection. The following table summarizes the electronics and DAQ parameters chosen to accomplish these goals. More details can be found in Chapter 10.

\begin{tabular}{|l|l|l|}
\hline \multicolumn{2}{|c|}{ Electronics \& DAQ Requirements } \\
\hline \multicolumn{1}{|c|}{ Parameter } & \multicolumn{1}{|c|}{ Value } & \multicolumn{1}{c|}{ Motivation } \\
\hline Dynamic Range & $<500: 1 \quad \begin{array}{l}\text { ENysics signal dynamic range * 10:1 } \\
\text { signal/noise requirement }\end{array}$ \\
\hline Noise & shaper peaking time & $\begin{array}{l}\text { Distinguish 3 fC wire signal (1 MIP) } \\
\text { from noise with high efficiency at the } \\
\text { longest drift time (1.6 ms) with an } \\
\text { electron lifetime of 1.6 ms. }\end{array}$ \\
\hline $\begin{array}{l}\text { Beam trigger readout } \\
\text { time }\end{array}$ & $4.8 \mathrm{~ms}$ & $\begin{array}{l}\text { The TPC drift time is 1.6 ms. Samples } \\
\text { are taken 1.6 ms before and 1.6 ms } \\
\text { after a beam spill to reconstruct out- } \\
\text { of-time cosmic muons }\end{array}$ \\
\hline Shaper peaking time & $\sim 1 \mu \mathrm{s}$ & $\begin{array}{l}\text { The average electron diffusion over a } \\
\text { the drift distance is } \sim 1.4 \text { mm = } 1 \mu \mathrm{s} .\end{array}$ \\
\hline ADC sampling rate & $\sim 2 \mathrm{MHz}$ & $\begin{array}{l}\text { The sampling rate should be at least } \\
\text { 4/( shaper peaking time). }\end{array}$ \\
\hline ADC resolution & $\sim 12$ bit & $\begin{array}{l}\text { Minimize the rate of ADC overflow for } \\
\text { low momentum, highly ionizing } \\
\text { particles }\end{array}$ \\
\hline Data buffer storage & $\begin{array}{l}\text { Accelerator events: none } \\
\text { Supernova events: one hour }\end{array}$ & $\begin{array}{l}\text { Sufficient time for supernova } \\
\text { notification by SNEWS }\end{array}$ \\
\hline
\end{tabular}




\subsection{Detector Building Requirements}

The LArTF detector building for MicroBooNE, its first tenant, must allow the experiment to be operated reliably and safely in a cost-effective manner. The following table summarizes the design parameters chosen to achieve these goals. Chapter 5 provides more details.

\begin{tabular}{|c|c|c|}
\hline \multicolumn{3}{|c|}{ Detector Building Requirements } \\
\hline Parameter & Value & Motivation \\
\hline Location & $\begin{array}{l}\text { Same plan and elevation of } \\
\text { MiniBooNE, } 240^{\prime} \text { upstream. }\end{array}$ & $\begin{array}{l}\text { Replicate beam conditions of } \\
\text { MiniBooNE. }\end{array}$ \\
\hline Architecture & "Cylinder and shed" & Cost. \\
\hline Roof construction & $\begin{array}{l}\text { Removable pre-cast panel, } \\
\text { weather-tight. }\end{array}$ & $\begin{array}{l}\text { Removal/replacement for } \\
\text { detector installation. }\end{array}$ \\
\hline Roof load capacity & $\begin{array}{l}\text { Up to } 10^{\prime} \text { of earth equivalent } \\
\text { shielding. } \\
\text { No shielding initially }\end{array}$ & $\begin{array}{l}\text { Cosmic ray shielding can be } \\
\text { added using shield blocks as } \\
\text { needed. }\end{array}$ \\
\hline Levels & $\begin{array}{l}706^{\prime} 0^{\prime \prime} / 707^{\prime} 3^{\prime \prime}, \\
732^{\prime} 4^{\prime \prime} / 733^{\prime} 5^{\prime \prime} \\
746^{\prime}\end{array}$ & $\begin{array}{l}\text { Refrigeration plant, } \\
\text { Detector readout service, } \\
\text { Loading dock, electronics. }\end{array}$ \\
\hline Building size & $1860 \mathrm{sq} f \mathrm{ft}$ at $706^{\prime}$ level & $\begin{array}{l}\text { Detector plus services plus } \\
\text { ODH-zero emergency exit }\end{array}$ \\
\hline Electronics "shed" & $335 \mathrm{sq} \mathrm{ft}$ & $\begin{array}{l}\text { Adequate capacity, limit cable } \\
\text { runs to } \sim 100 \mathrm{ft} \text {. }\end{array}$ \\
\hline Enclosed access stairs & $\begin{array}{l}63 \times 39^{\prime \prime} \text { stairs from } 745^{\prime} \text { to } \\
706^{\prime} \text { at ODH-0 with } 0.5-1.0^{\prime \prime} \\
\mathrm{H} 2 \mathrm{O} \text { overpressure. }\end{array}$ & Emergency exit from all levels. \\
\hline Crane & 5 ton & Refrigeration equipment. \\
\hline Electrical power & $\begin{array}{l}\text { Building +Clean: } 500 \text { KVA } \\
+150 \text { KVA transformers. }\end{array}$ & Services and electronics. \\
\hline Backup power & $\begin{array}{l}150 \mathrm{~kW} \text { natural gas with } \\
\text { automatic transfer switch }\end{array}$ & $\begin{array}{l}\text { Cryogenics monitoring, HVAC } \\
\text { for ODH escape. }\end{array}$ \\
\hline Fire suppression & $\begin{array}{l}\text { Sprinkler mounting fits with } \\
\text { removable roof. }\end{array}$ & Code. \\
\hline $\mathrm{ICW}$ & $\begin{array}{l}30 \mathrm{gpm}, 2 \text { degree working } \\
\text { temperature drop. }\end{array}$ & Cooling. \\
\hline HVAC & Conventional, 4 zones & $\begin{array}{l}\text { Electronics, ODH-0 emergency } \\
\text { exit, } \mathrm{ODH}-1 \text { in cylinder in } \\
\text { accident condition. }\end{array}$ \\
\hline Air exchange rate & $\begin{array}{l}7500 \text { CFM in LAr spill, } \\
\text { else set for occupancy }=20\end{array}$ & $\begin{array}{l}\text { Maintain ODH-1 or better in } \\
\text { ODH accident. }\end{array}$ \\
\hline Argon spill volume & $\begin{array}{l}\text { Insulated (floor and walls) } \\
\text { volume under grating } 15^{\prime \prime} \\
\text { above } 706^{\prime} \text { level. }\end{array}$ & $\begin{array}{l}\text { Mitigate ODH conditions in } \\
\text { accident. }\end{array}$ \\
\hline Occupancy & $\begin{array}{l}<20 \text { during installation } \\
\sim 0 \text { during operation }\end{array}$ & $\begin{array}{l}\text { Adequate for anticipated tasks. } \\
\text { No sanitary facilities. }\end{array}$ \\
\hline External access, storage & $\begin{array}{l}\text { Paved road to loading dock, } \\
\text { paved tanker parking area }\end{array}$ & Access, cryogen storage. \\
\hline
\end{tabular}




\section{Project Overview (WBS 1.1)}

\subsection{Scientific Objectives}

The three major objectives of the MicroBooNE experiment combine immediate scientific interests with technological innovations for the long term. The two primary scientific goals are to resolve the source of the MiniBooNE low energy excess and to measure a number of neutrino cross sections in the Booster Neutrino Beam (BNB) energy range. A third important aim of MicroBooNE concerns detector development. Building the MicroBooNE detector will provide technical experience in moderate-size liquid argon time projection chamber (LArTPC) detector construction, experience which will be directly applicable to the design of multi-hundred-ton LArTPC detectors. The design of the MicroBooNE experiment balances the detector R\&D objectives against timely and precise physics results to assure success on both the scientific and technological fronts. These are the primary design-drivers of the experiment. The scientific goals of MicroBooNE, as well as those of future multi-kiloton muon neutrino to electron neutrino oscillations experiments, rely on particular and largely unparalleled capabilities of LArTPC technology, namely, the precise differentiation between electrons and photons, and high resolution of particle tracks, which allows detection of nuclear debris.

Further details of the MicroBooNE experiment's planned measurements and expected sensitivity can be found in the initial experiment proposal ${ }^{1}$, the proposal addendum ${ }^{2}$, and the conceptual design report $(\mathrm{CDR})^{3}$. The $\mathrm{CDR}$ and this technical design report (TDR) contain details of the detector design. The MicroBooNE Detector Parameters Book gives specific design criteria for the experiment.

\subsection{Project Scope}

The MicroBooNE project has been created to manage and carry out the construction of the liquid argon (LAr) detector for the MicroBooNE experiment. MicroBooNE will be located in the Booster Neutrino Beam line, in a new enclosure to be constructed immediately upstream of the MiniBooNE detector hall. Construction of this enclosure falls within the scope of the MicroBooNE project. The main detector elements include the LAr cryostat and the cryogenic system required to cool and purify the LAr; the time projection chamber and a photomultiplier array, both installed inside the cryostat; and the electronics, readout system, and data acquisition system. The project scope includes the resources required to assemble the detector elements and install them in the experimental enclosure. The project successfully concludes with the detector installed in the new enclosure and ready to be filled with LAr. The following sections describe the specific project completion deliverables. 


\subsection{Work Breakdown Structure (WBS)}

The MicroBooNE project (WBS 1.0) contains ten Level 2 (L2) sub-projects established to manage the work required to construct the LArTPC for the MicroBooNE experiment. Table 4.1 presents the WBS dictionary.

\begin{tabular}{|c|c|c|}
\hline WBS & Title & Description \\
\hline & MicroBooNE Project & $\begin{array}{l}\text { LArTPC Detector positioned in the Booster Neutrino } \\
\text { Beam. }\end{array}$ \\
\hline 1.1 & Project Management & $\begin{array}{l}\text { Project Management support; includes level of effort for } \\
\text { the project office and M\&S for travel, contract personnel } \\
\text { for project management and office supplies including } \\
\text { software licenses. }\end{array}$ \\
\hline 1.2 & $\begin{array}{l}\text { Cryogenics and } \\
\text { Purification }\end{array}$ & $\begin{array}{l}\text { Cryogenic plant, including cryogen delivery and storage, } \\
\text { argon circulation and purification, vessel filling, liquid } \\
\text { nitrogen system, and the cryogenic system monitors and } \\
\text { controls. }\end{array}$ \\
\hline 1.3 & Cryostat & $\begin{array}{l}\text { Cryogenic vessel, with internal and external fixtures and } \\
\text { penetrations for the support of detector and cryogenic } \\
\text { elements; the vessel mechanical support; specification of } \\
\text { the vessel insulation. }\end{array}$ \\
\hline 1.4 & $\begin{array}{l}\text { Time Projection } \\
\text { Chamber }\end{array}$ & $\begin{array}{l}\text { LArTPC system located inside the cryogenic vessel. This } \\
\text { includes the anode plane frame, wires, wire connector } \\
\text { boards, cathode plane, field cage tubing, and structural } \\
\text { support fixtures. [NSF funded] }\end{array}$ \\
\hline 1.5 & FEE and Readout & $\begin{array}{l}\text { 1) Cold analog electronics mounted on the TPC inside } \\
\text { the vessel; the feedthroughs; Faraday cages and cabling } \\
\text { for TPC readout signals and power; warm support } \\
\text { electronics located on the vessel; digitization ; [DOE } \\
\text { project] } \\
\text { 2) Readout systems and data collection and formatting, } \\
\text { triggers and timing; HV supply and cables to the splitter } \\
\text { for the PMT system. [NSF grant to Columbia] }\end{array}$ \\
\hline 1.6 & $\begin{array}{l}\text { Experiment } \\
\text { Infrastructure }\end{array}$ & $\begin{array}{l}\text { Infrastructure systems needed to support the operation } \\
\text { of the experiment, including preparation of the } \\
\text { experiment site and experiment outfitting such as } \\
\text { electrical, HVAC, safety, and communications systems. }\end{array}$ \\
\hline
\end{tabular}




\begin{tabular}{|c|l|l|}
\hline 1.7 & Systems Installation & $\begin{array}{l}\text { Assemble detector components into a whole LAr TPC } \\
\text { and PMT active detector. Included is the pre-installation } \\
\text { vessel preparation, installation of the detector, the } \\
\text { cryogenic plant, and experiment readout and monitoring } \\
\text { systems in the experiment enclosure. }\end{array}$ \\
\hline $\mathbf{1 . 8}$ & PMT System & $\begin{array}{l}\text { Photomultiplier Tube (PMT) system, including the } \\
\text { detector elements located inside the vessel: PMTs and } \\
\text { bases, wavelength-shifting plates and cables to a feed- } \\
\text { thru; exterior cables to a HV-signal splitter; cables to the } \\
\text { PMT readout crate. }\end{array}$ \\
\hline $\mathbf{1 . 9}$ & $\begin{array}{l}\text { DAQ, Monitoring, and } \\
\text { Control }\end{array}$ & $\begin{array}{l}\text { Hardware and software required for Data Acquisition, } \\
\text { Detector Monitoring, and Control. }\end{array}$ \\
\hline $\mathbf{1 . 1 0}$ & $\begin{array}{l}\text { Detector Assembly } \\
\text { Integration }\end{array}$ & $\begin{array}{l}\text { Design effort for the overall integration of systems into } \\
\text { the detector; LArTPC high voltage system including feed- } \\
\text { thru and power supply; LArTPC installation fixtures; PMT } \\
\text { feedthrough; and PMT support rack. }\end{array}$ \\
\hline
\end{tabular}

Table 4.1 MicroBooNE WBS dictionary.

\subsection{Project Stages and Approvals}

\subsubsection{CD-0 (Approve Mission Need)}

The MicroBooNE project follows from the CD-0 document entitled "Mission Need Statement for Large Liquid Argon Detector for Neutrino Physics." The DOE Office of High Energy Physics issued this document on September 10, 2009; it can be accessed as MicroBooNE DocDB-593. The CD-0 document proposed three alternatives for meeting the mission need:

Alternative 1 - Do nothing.

Alternative 2 - Build a 100 ton scale liquid argon neutrino detector.

Alternative 3 - Collaborate with other liquid argon TPC development efforts.

At the time of the mission need approval, Alternative 2 emerged as the favored option, based on the Fermilab MicroBooNE proposal approved by the Fermilab Director in July 2008.

\subsubsection{CD-1 (Approve Alternative Selection and Cost Range)}

The DOE Office of Science Associate Director for High Energy Physics granted CD-1 approval for the MicroBooNE Project, based on the detector described in the MicroBooNE proposal (Alternative 2), on July 9, 2010. This approval document is available as MicroBooNE DocDB987. The total project cost (TPC) was set to be in the range $18.8-20.0$ million dollars, in then- 
year dollars. The funding profile spans the fiscal years FY2010-2013, with an anticipated project construction start set for the fourth quarter of FY2011 at the time of the CD-1 approval.

\subsubsection{CD-2 (Approve Performance Baseline) and CD-3 (Approve Start of Construction)}

The systems described in this TDR have been developed to the level that a project baseline will be established based on these designs. The Project team received CD-2 approval in the fourth quarter of FY2011, along with CD-3a approval for long lead-time procurements shortly thereafter. These approvals resulted in the release of adequate funding to complete all final designs, and a corresponding request for CD-3b approval in the first quarter of FY2012.

\subsubsection{CD-4 (Approve Project Closeout)}

The CD-4 deliverables for the MicroBooNE project, described in more detail below, comprise:

- A complete and operational detector facility.

- All safety systems operational.

- An installed detector, with all detector and readout sub-system checkouts completed.

- Cryogenic plant tests with liquid argon completed and ready for initial detector fill.

Achievement of all four of these objectives leads to a detector ready for filling with liquid argon.

\subsubsection{Detector facility complete and operational}

The detector facility will be built under contract with external firms. The contract will include delivery of electric power, HVAC, and fire protection systems. Upon completion of the contract, Fermilab personnel will install lab-specific services such as computer networks, FIRUS, ACNET, and telephones. The facility reaches a complete and operational state only after all of these systems become operational.

\subsubsection{All safety systems operational}

The MicroBooNE detector hall must by necessity become an oxygen deficiency hazard (ODH) area that contains ODH alarms connected to Fermilab's FIRUS system. The MicroBooNE cryogenics controls system will utilize a parallel method of monitoring and reporting these alarms to the experiment. The readout electronics racks will use rack protection systems (auto shut-off of power in case of smoke or fire), which will also have a method of reporting their status to the experiment. All of these safety systems, plus others (operations procedures, for example) will become a part of the Operational Readiness Clearance (ORC), as defined by the Fermilab Particle Physics Division (PPD) Environmental, Safety, and Health (ES\&H) section. Safety systems having achieved operational status means that all of these systems have received their partial clearance (pORC), and that the detector as a whole has ORC. Granting of pORC on the cryogenics implies that the Fermilab Cryogenics Safety Committee has approved operation of the system. 


\subsubsection{Detector installation completed}

"Detector installation completed" means: TPC, photodetectors, and cryogenics monitoring installed inside the vessel; all related detector cabling finished; the vessel end cap sealed; all external electronics and power supplies installed; all detector monitoring systems installed; data acquisition and handling systems installed; and all cryogenic systems installed.

Details of sub-system checkouts remain under discussion within the collaboration. They will be defined more fully by the time of the Baseline Review (CD-2) in the form of a set of benchmark goals set by the sub-system groups.

\subsubsection{Cryogenic plant tests with liquid argon completed}

Cryogenic plant tests with liquid argon include verification of the operability of the condenser cryogenic pumps. Additional details will to be specified under the assumption everything that can be tested will be tested up to the point where turning on the "fill" valve represents the only remaining task needed to initiate filling the vessel.

\subsubsection{Items not included for $C D-4$}

The CD-4 goals do not include filling the detector with cryogens. The following provides a rationale for this decision: An important element in MicroBooNE's R\&D program will be a demonstration of filling without evacuation (purge filling) that will result in a LAr purity level sufficient for physics operations. This demonstration will likely require several fill and purge cycles. Each such cycle may create "cold shock" to the vessel and its penetration flanges, and these shocks may cause leaks at the flanges. While an estimate of two months for this initial demonstration was obtained in the experiment proposal, more conservative estimates would allow more time. None of these estimates include time to detect and repair leaks. Because time may be needed to repair any leaks produced by cold shocks, the schedule for the entire purge-fill demonstration remains at present difficult to predict. Therefore the Project's CD-4 goals do not include this activity.

\subsection{Project Management}

Management Plans for the MicroBooNE Project include the Project Execution Plan, prepared by the DOE Federal Project Director and approved by the Director of the DOE Office of High Energy Physics, and the Project Management Plan, prepared by the MicroBooNE Project Manager and approved by the DOE Federal Project Director. Both of these documents can be found in the MicroBooNE document database (MicroBooNE DocDB 1556 and 1557 for the Project Execution Plan and Project Management Plan, respectively.)

\footnotetext{
${ }^{1}$ The MicroBooNE Collaboration, A Proposal for a New Experiment Using the Booster and NuMI Neutrino Beamlines: MicroBooNE, FERMILAB-PROPOSAL-974, submitted to the Fermilab Physics Advisory Committee, October 2007, http://www-microboone.fnal.gov/Documents/MicroBooNE 10152007.pdf.

${ }^{2}$ The MicroBooNE Collaboration, The MicroBooNE Proposal Addendum, March 3, 2008, http://www-microboone.fnal.gov/Documents/MicroBooNE addendum/MicroBooNEAddendum 030308.pdf.

${ }^{3}$ The MicroBooNE Collaboration, Conceptual Design Report, October 2009,
} 
http://www-microboone.fnal.gov/project/cd1_dr/MicroBooNE_CDR.pdf. 


\section{Experiment Infrastructure (WBS 1.6)}

\subsection{Introduction}

MicroBooNE will be housed in a new enclosure, the Liquid Argon Test Facility (LArTF), located upstream of the MiniBooNE on the axis of the Booster Neutrino Beam (BNB). Its design duplicates much of that of the building that houses MiniBooNE.

The first complete proposal developed for housing MicroBooNE in fact followed a directive to reuse the MiniBooNE facility after the removal of the MiniBooNE Detector. This involved designing modifications of the enclosure for MicroBooNE and developing cost estimates for removal of the MiniBooNE detector. The Fermilab decision to continue MiniBooNE data taking resulted in a review that rejected the proposal for reuse of the MiniBooNE building and construction instead redirected design efforts towards the new LArTF. However, much of the technical development for the rejected proposal remains relevant for LArTF, and the basic cylindrical structure of the MiniBooNE facility provides the most cost effective alternative for new underground construction.

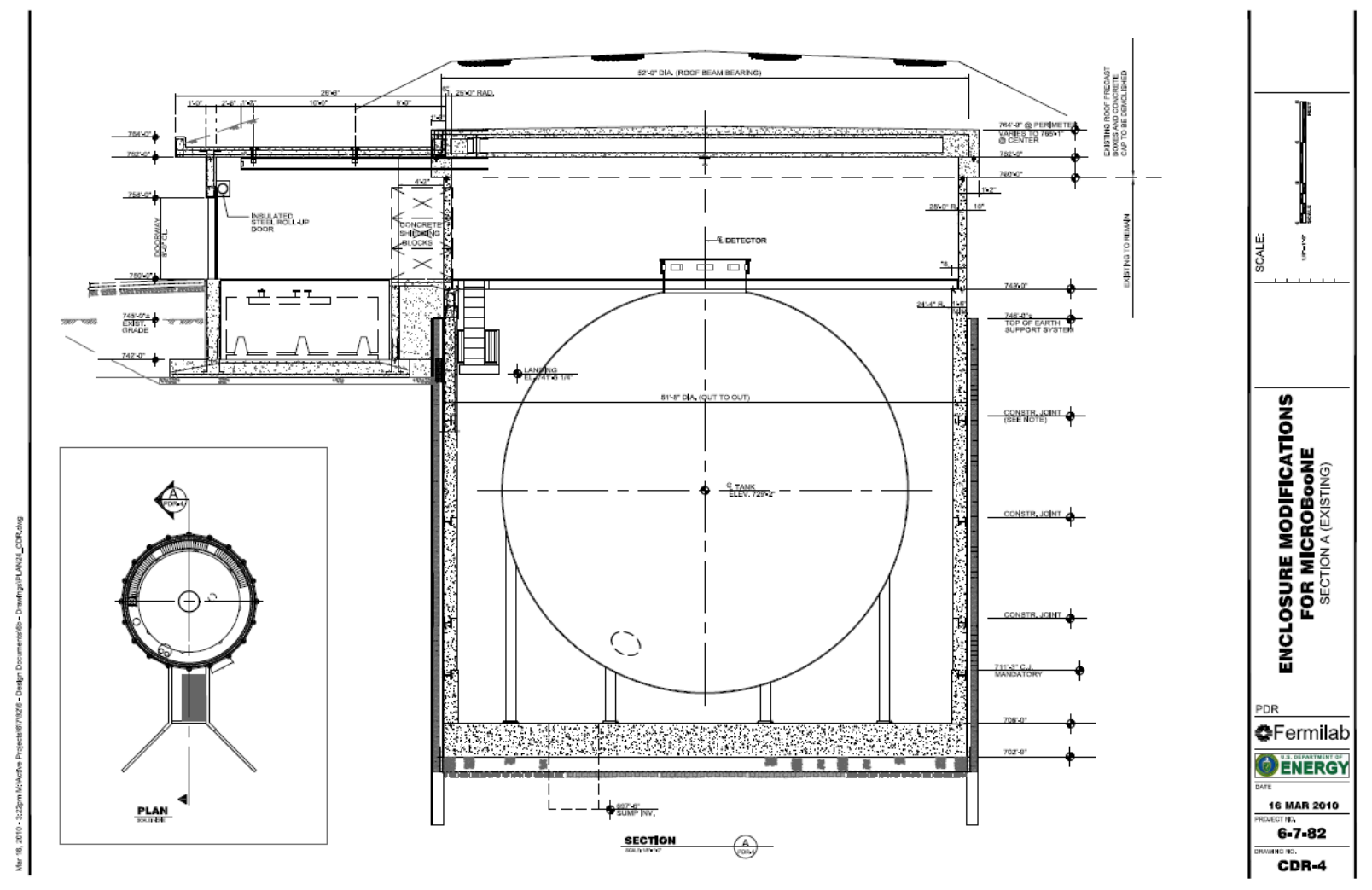

Figure 5.1 The existing MiniBooNE Detector in its concrete cylinder.

The existing MiniBooNE facility, constructed in 1999-2000, consists of a 50' outside diameter, 48' '" inside diameter vertical concrete cylinder about 44 feet deep with a floor at elevation 706 
feet. A sump at elevation 706 removes ground water to the surface. Two $150 \mathrm{kVA}$ transformers fed from Fermilab feeder 44 provide electrical power. An integral roof covers the cylinder and supports approximately 10 feet of earth shielding cover.

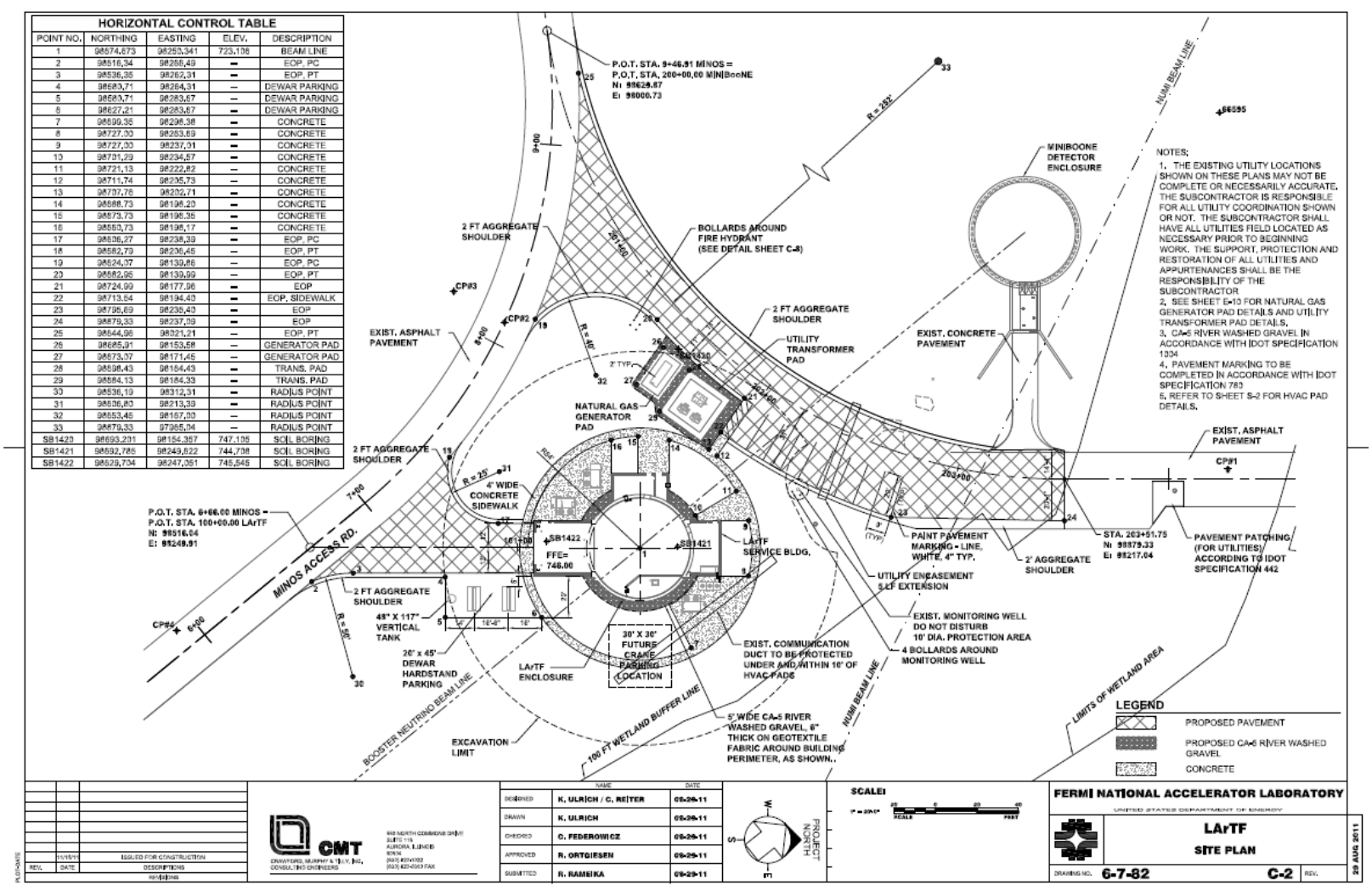

Figure 5.2 Location of the LArTF "Cylinder and Shed" upstream of MiniBooNE.

For the proposed location of the LArTF new utilities must be brought from the existing utility corridor, including $13.8 \mathrm{kV}$ electrical service, industrial cooling water (ICW), and natural gas. New construction will not disturb MiniBooNE except for outages during utility connections.

Five entries will be provided to the LArTF at grade: a delivery area with truck access, an electronics room, a door into one of two vertical stair columns to access the various below grade levels, a door into the second stair column through an outside covered entry, and an entry into the fire protection control room. A roof with a removable slot sufficient to permit the entry of the MicroBooNE detector cryostat must be provided. The roof must have the carrying capacity for 10 feet of earth equivalent material, which could be added at a later time. Electrical service will include one $150 \mathrm{kVA}$ transformer and one $500 \mathrm{kVA}$ transformer. Provision for safe egress in the event of an ODH incident must be provided. Supports for the cryostat, readout electronics, and refrigeration equipment must be constructed. Adequate ICW for process cooling must be provided. 

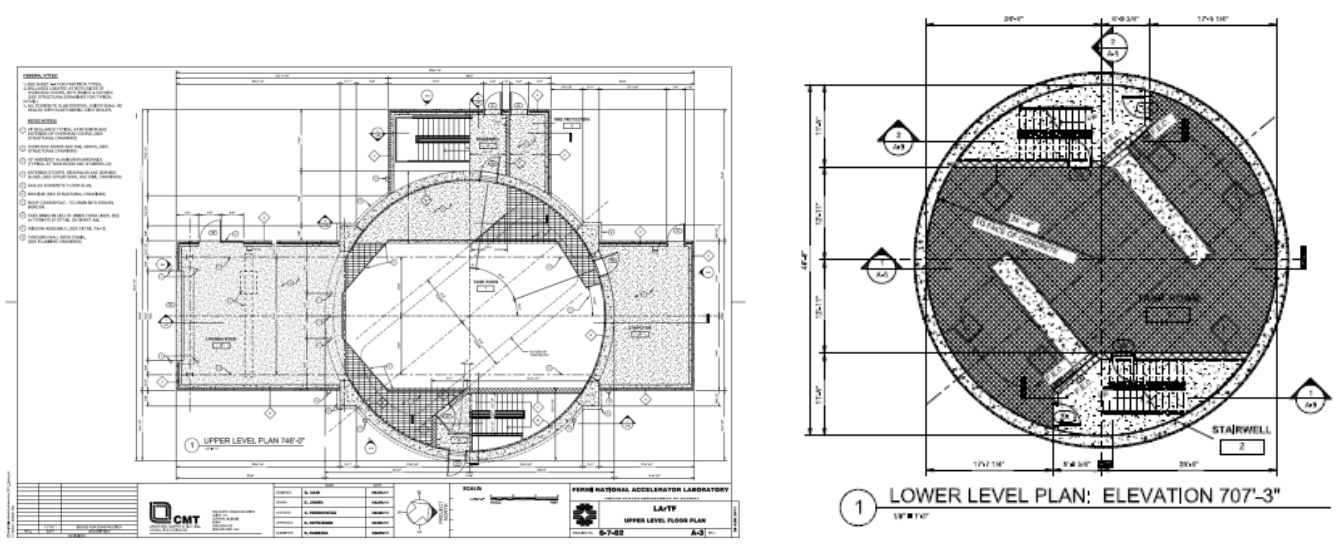

Figure 5.3 Plan view of the "cylinder and sheds" facility showing the loading dock area (left extension), electronics room (right extension), and cylinder with two stair columns.

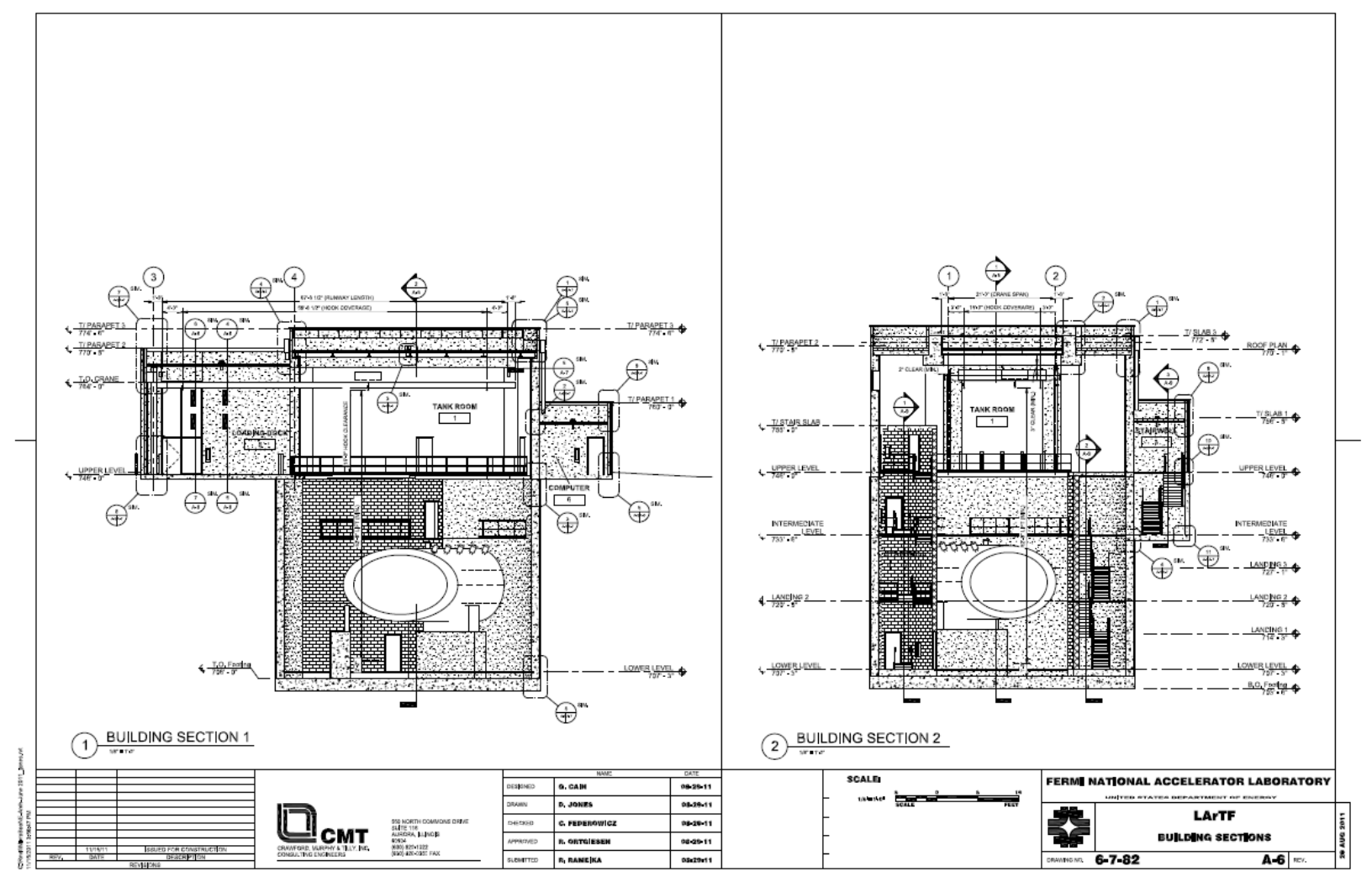

Figure 5.4 Elevation views showing MicroBooNE Detector in cylindrical pit. 


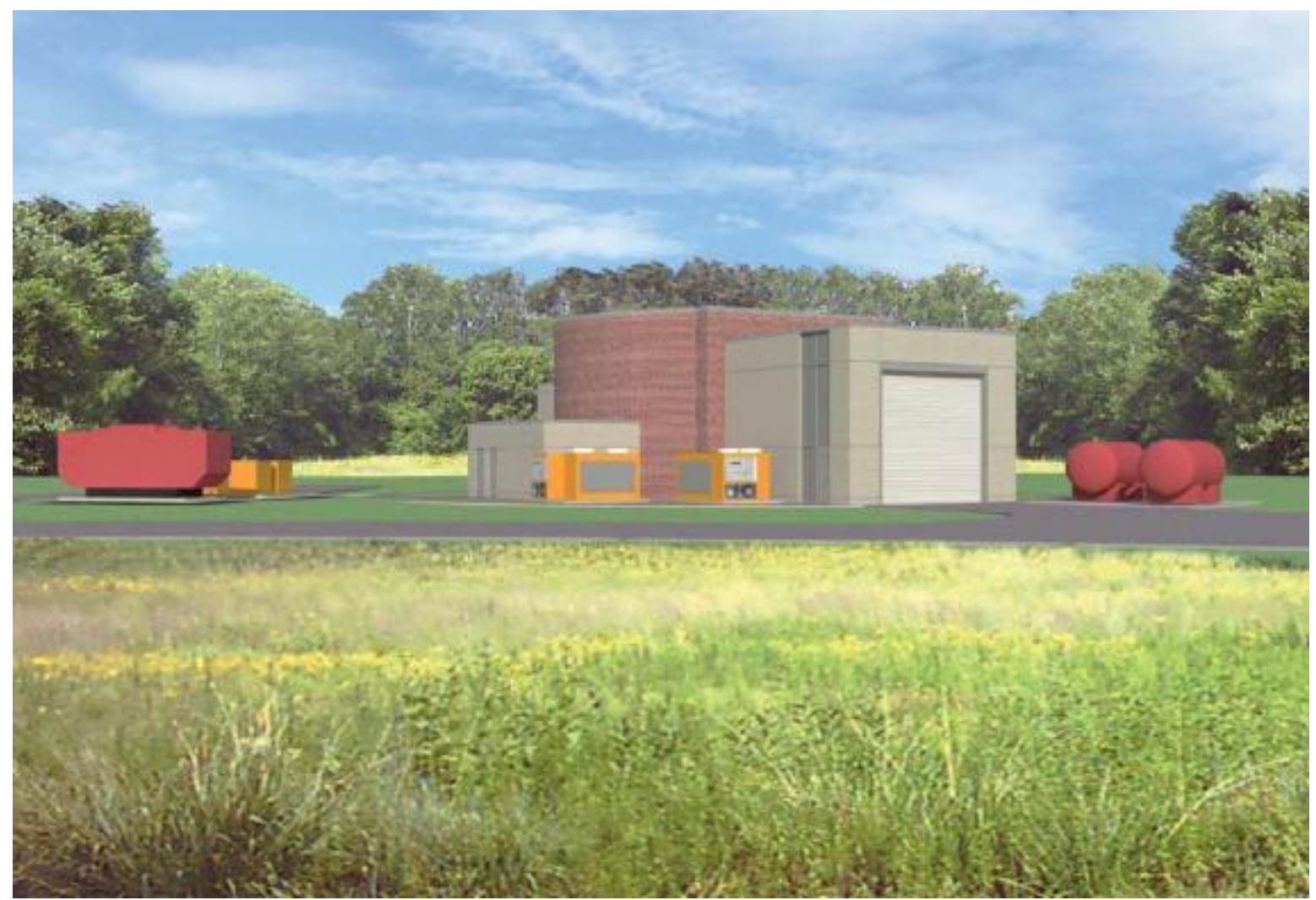

Figure 5.5 Three-dimensional renderings of the LArTF cylinder and sheds loading dock and stairs (NW view).

\subsection{Detailed Specifications for the new enclosure}

The following list summarizes specifications developed for the LArTF "Cylinder and Shed" Facility:

1. The location of the liquid argon tank with the detector shall be "close" to the MiniBooNE detector center on the existing BNB beamline in both plan and elevation, but approximately 240' upstream of the MiniBooNE Facility. The MicroBooNE detector center will lie at the following coordinates: $\mathrm{X}[\mathrm{ft}]=98250.341, \mathrm{Y}[\mathrm{ft}]=98674.673$, and $\mathrm{H}[\mathrm{ft}]=723.106$ (earth curvature corrected). Computations show this MicroBooNE cylinder center to be collinear with the BNB decay pipe center, as ascertained by best fit line through the as-found points during construction quality control, and 240 feet upstream of the MiniBooNE Detector center.

2. The basic cylinder and two attached "shed" structures at grade house the detector tank, supporting refrigeration, readout electronics, and delivery area. The depth of the cylinder equals that of the existing MiniBooNE cylinder. The new facility requires three working elevations dictated by the refrigeration plant under the cryostat and the beamline center, as depicted in Figure 5.4:

- 706'0"/707'3" ("Refrigeration Plant Level");

- 732'4"/733'5" ("Detector Readout Service Level”);

- 746'("Loading Dock Level/Electronics Room Level”). 
3. New taps must be made to existing ICW, natural gas, electrical, and domestic water (DWS) services in the nearby utility corridor (See Figure 5.2). An additional ICW line may be needed to create a "loop" so that ICW can be used for process chilling and then discharged back into the ICW system.

4. The insertion of the argon tank/detector shall be by an exterior movable crane through removable roof sections. A concrete "collar" section will be extended above grade to support the roof. The dimensions of the rectangular opening will be 44 '2" by 23 ', forming a 530 " by 276 " clear opening.

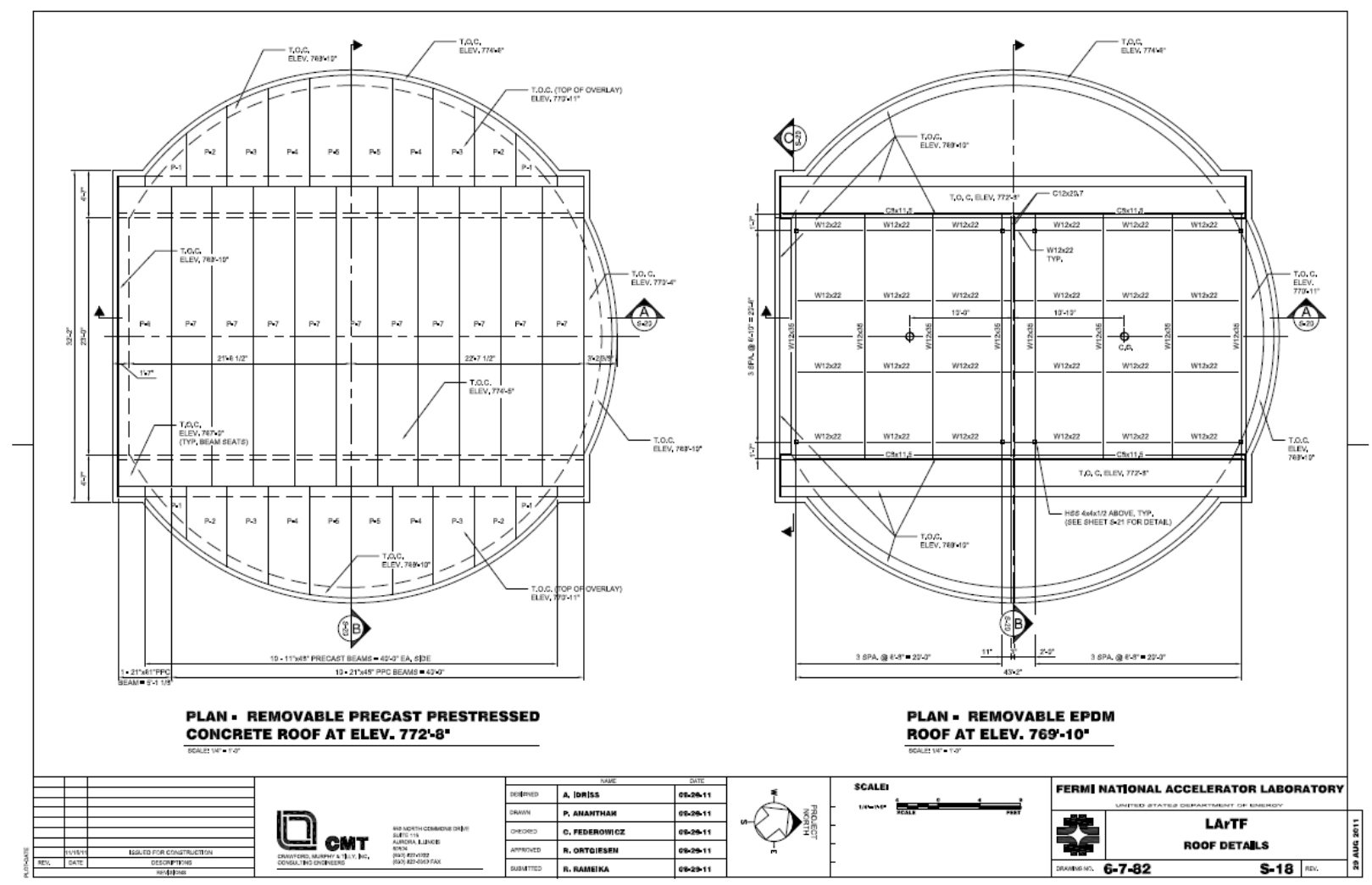

Figure 5.6 The new roof with the central removable slabs in place(left) and the framing for the removable weathertight roof (right).

5. The existing MiniBooNE facility roof supports approximately 10 foot of earth shielding to shield the MiniBooNE detector from cosmic rays. The LArTF design has no roof shielding; however, provision has been made so as not to exclude additional shielding at a later date. The pre-cast panel roof and any shielding system must be made weather tight.

6. A total of $1860 \mathrm{sq} \mathrm{ft}$ becomes available at the elevation 706 level. About $410 \mathrm{sq} \mathrm{ft}$ will be used to provide two emergency exit stairways that are over pressurized to maintain an ODHzero classification. Of the remaining approximately $1450 \mathrm{sq} \mathrm{ft}$ at elevation, 706 will be available for refrigeration equipment and other support services for the liquid argon cryostat. 


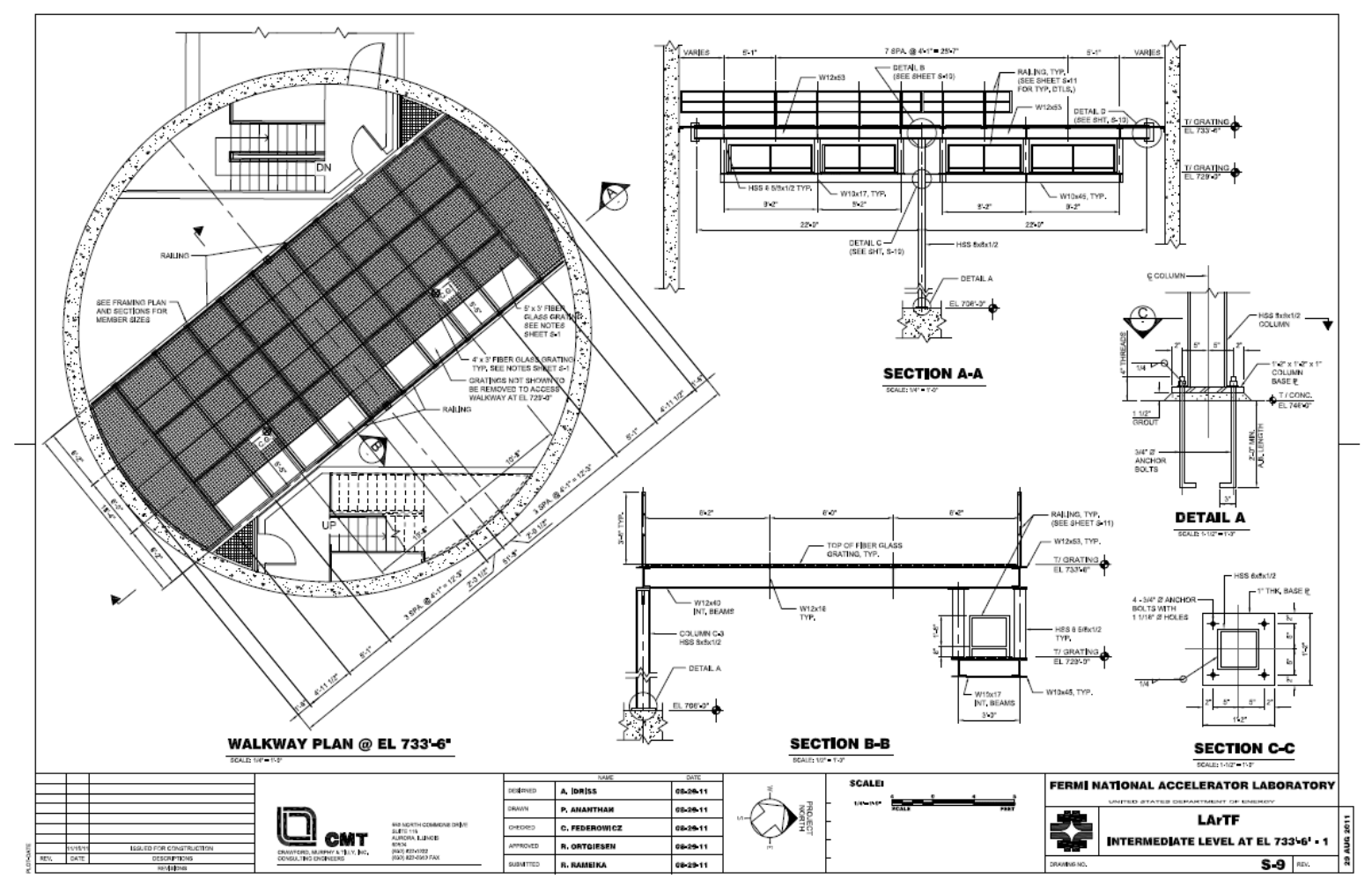

Figure 5.7 Plan views of the pit floor and the walkways at the top of the cryostat.

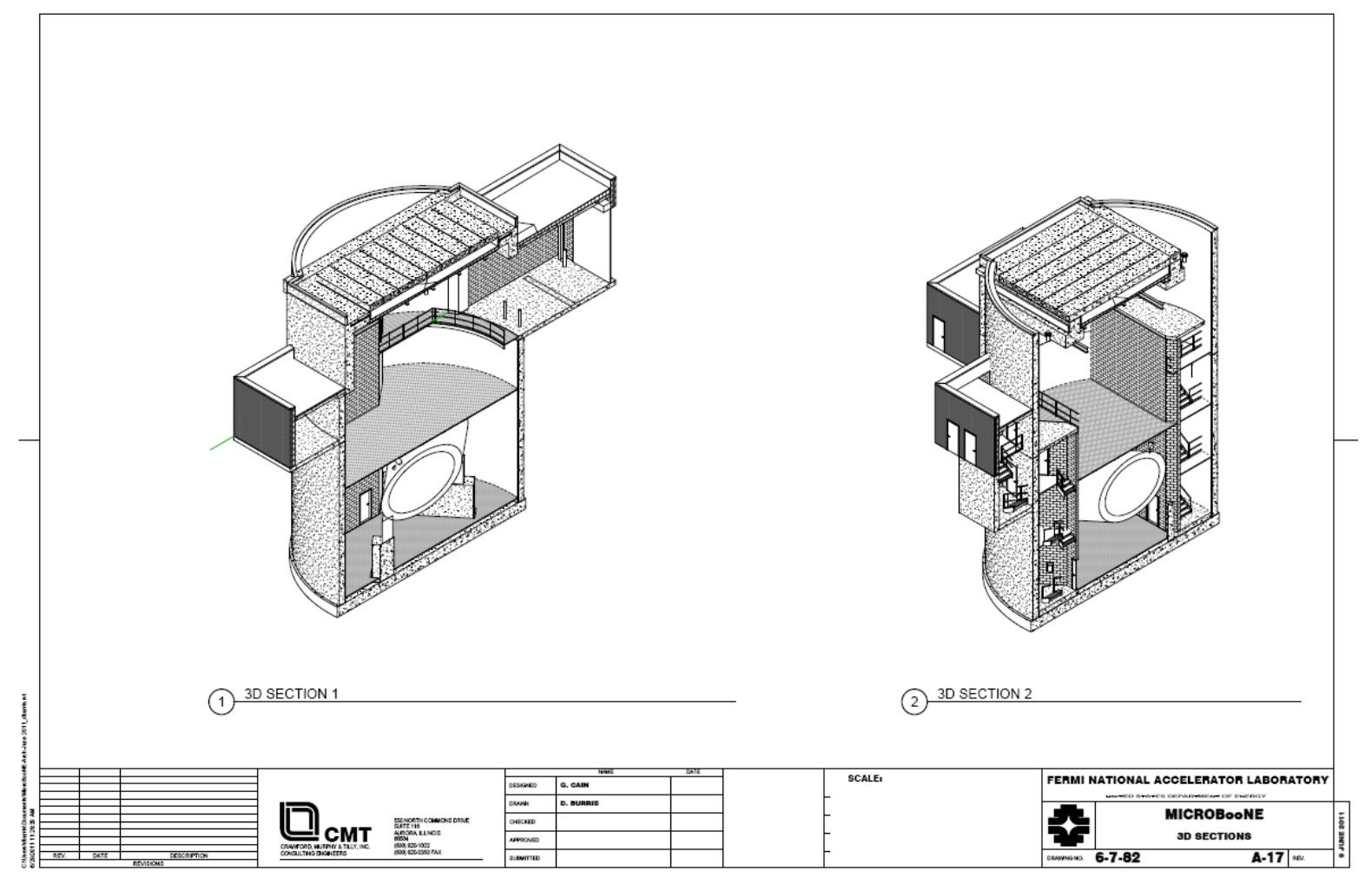

Figure 5.8 Three-dimensional cuts of the cylinder and emergency stairs. (Note that this rendering contains an error. The floor shown at $\mathbf{7 3 3}$ does not cover the entire area as shown here.) 
7. A five ton capacity crane will be provided to move refrigeration equipment between the loading dock level and the 706' elevation level. There will be a 15' pick height above elevation $746^{\prime}$, and the hook will descend to elevation $706^{\prime}$ requiring a total hook travel of approximately $55^{\prime}$.

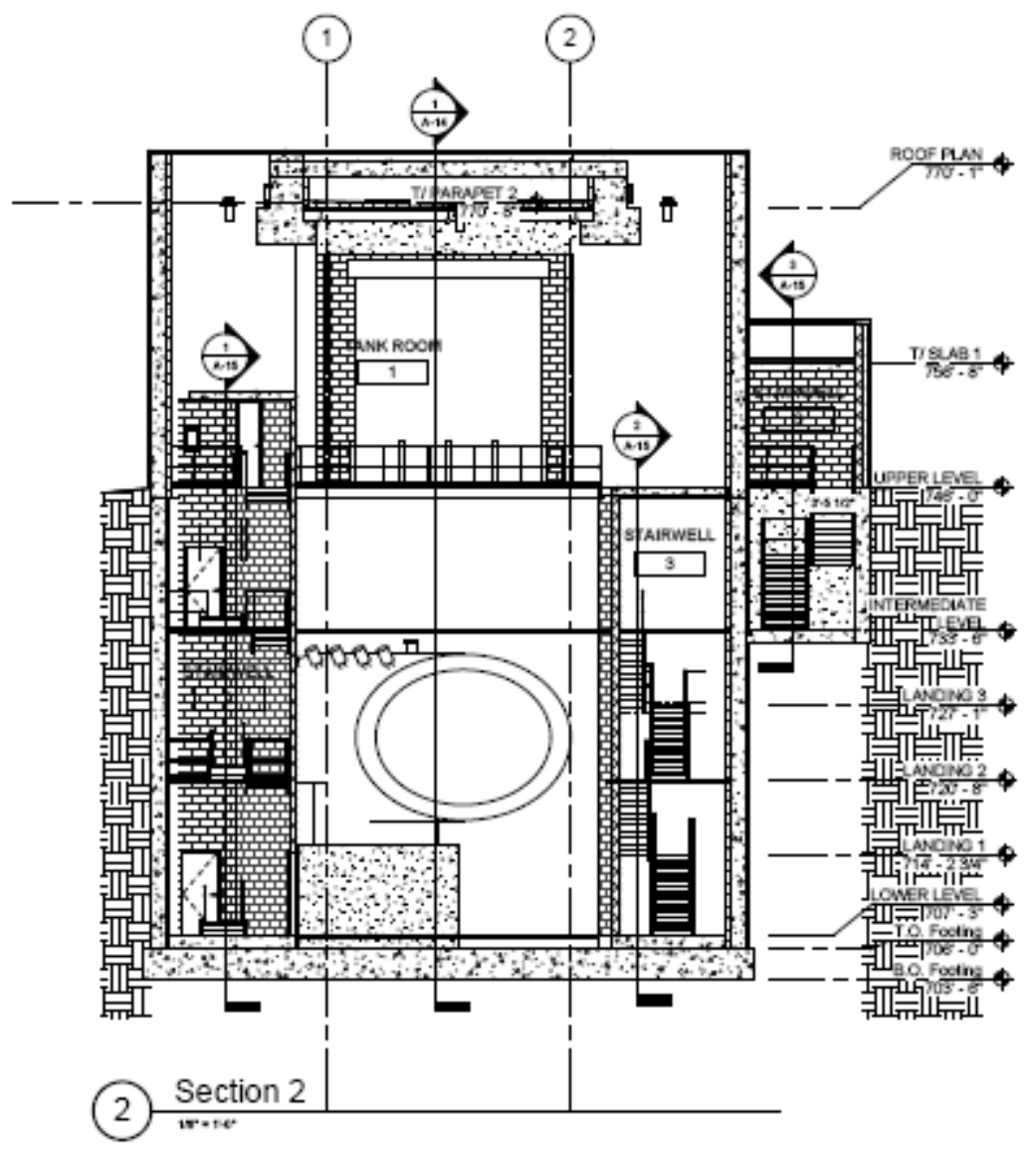

Figure 5.9 In Section 2 the emergency stair enclosures are shown on the left and right sides of the pit, and the 5 ton crane is shown over the central portion of the pit.

8. The area of the "Loading Dock" at elevation 746' will be 21 'x25', which comprises $525 \mathrm{sq}$ $\mathrm{ft}$. Crane coverage within this area will be approximately $16^{\prime} \times 22^{\prime}=352 \mathrm{sq} \mathrm{ft}$ at elevation $745^{\prime}$, and the crane will also cover about $16^{\prime} \times 40^{\prime}=640$ sq $\mathrm{ft}$ over elevation $706^{\prime}$. There will be a paved road to the loading dock, and paved tube tanker parking for a pair of tube tankers.

9. There will be two enclosed, over pressurized access stairs from elevation 746' down to elevation 706'. The elevation change up to grade will be 40' (65 steps) above elevation 706'. Emergency egress within the ODH zero stairways will therefore require a climb of about five stories. 
10. The electronics room will have an area of about $335 \mathrm{sq} \mathrm{ft}$. The electronics cable run from the detector tank to the electronics will be approximately 100' or less.

11. There will be four distinct HVAC regions:

- Area A, the electronics room;

- Area B, the loading dock;

- Area C, the over pressurized ODH “zero" exit stair columns to ground from the 706' elevation;

- Area D, the remaining spaces in the rest of the cylinder structure. These will be designed with sufficient exhaust fans such that in the event of an ODH accident the conditions should not exceed ODH-1.

12. An argon spill volume will be created with insulation under a grating built 15 " above the 706 elevation floor.

13. The argon spill vent air-exchange rate will be 7500 CFM. The standard non-accident air exchange rate will be consistent with s 20 person occupancy.

14. In the exit stairs the ODH "zero" region will be over-pressurized to be between $1 / 2$ " and 1" water.

15. Building electrical power will require a $500 \mathrm{kVA}$ transformer. Clean power will be provided by a $150 \mathrm{kVA}$ transformer. The electrical service motor control center will be located on the west wall of loading dock area.

16. A natural gas emergency power generator rated at $150 \mathrm{KW}$ is required, with an automatic transfer switch. This emergency service is sized for cryogenics monitoring and HVAC for ODH escape, etc.

17. Fire suppression with a sprinkler system, and fire alarms, will be provided. Mounting of the sprinkler pipes will be consistent with the removable roof requirement.

18. Conventional HVAC is specified for the four HVAC areas defined above:

- Area A ---72 F to 75 F, 50\% RH max, no min RH, (12 racks @25KW/rack);

- Area B---not conditioned, $65 \mathrm{~F}$ min;

- Area C---60 F to $80 \mathrm{~F}, 55 \% \mathrm{RH} \max$, no $\min \mathrm{RH}, 60 \mathrm{~F} \min , 1 / 2$ " to 1" water overpressure;

- Area D--- not conditioned, 55\% RH max, no min RH, 60F min, standard air exchange for 20 person occupancy, $7500 \mathrm{cfm}$ exhaust under ODH accident.

19. Process cooling water will be required with a $30 \mathrm{gpm}$ flow and 2 degree working temperature drop. An ICW loop will be created with discharge back to MINOS surface sump tank.

20. Cryogenic transfer piping mounts will be installed on the east wall of loading dock area.

21. Expected occupancy will be less than a maximum of twenty during installation, and approximately zero during operations. No sanitary facilities will be provided in the structure, implying that portable toilets are necessary.

22. Outside storage space must be sufficient for a LN2 tank and a small LAr Dewar. 


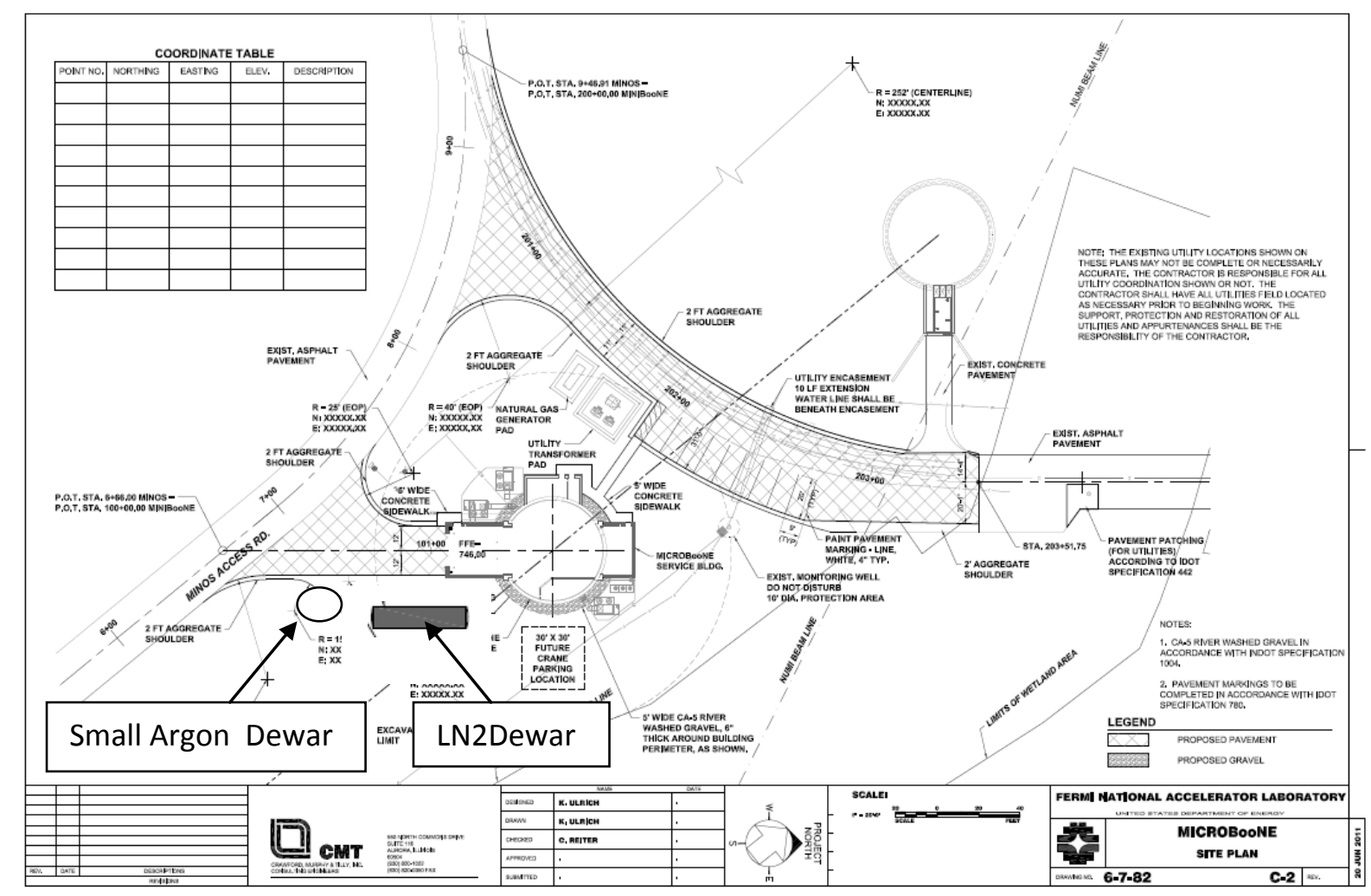

Figure 5.10 A plan view of the LArTF showing outside storage areas.

23. The new roof will be designed with easily removed precast beams, with only a central slot needed to be opened for delivery or service the cryostat.

24. Radiation interlocks will not be necessary; but ODH alarms will be installed.

25. Standard lighting specifications apply.

26. The walkways around the cryostat shown in Figure 5.7 will be modular in design with engineered lift points. The individual modular pieces will weigh less than 5 tons so that they may be lifted with the 5-ton crane and stored in the receiving area. This will permit the installation of the modular walkways before the delivery of the cryostat, and permit easy disassembly when the cryostat is ready to install, or after installation if the cryostat must ever be removed for servicing.

\subsection{Documentation}

MicroBooNE DocDB 1549 includes the construction drawings for the LArTF described in this chapter. A commercial architect and engineering firm, CMT, did the design and produced the construction drawings. The LArTF GPP construction work was bid and awarded to Whittaker Construction. A notice to proceed was issued December 12, 2011. Beneficial Occupancy is scheduled for March 2013, with construction completion expected June 28, 2013. 


\section{Cryogenics (WBS 1.2)}

\subsection{Introduction}

Use of liquid argon (LAr) as target and detection medium defines the key feature of MicroBooNE. When a charged particle passes through LAr, the argon emits detectable scintillation light, and the ionization produces electrons that can be drifted in electric fields over a distance of meters without significant dispersion and collected on wire chamber anodes. To exploit these properties fully, the argon must exist in appropriate chemical and thermodynamic states. It must stay sufficiently free of contaminants such as nitrogen and oxygen to avoid quenching of the scintillation light, and it must remain minimally contaminated by impurities such as oxygen and water to avoid losing drifting electrons to capture on these electronegative molecules. Temperature gradients in the liquid must remain small so that convection currents do not distort the drifting ionization trails; and, given the significant dependence of the electron drift velocity on temperature, the overall temperature variation in liquid must be controlled. The cryogenics team managed under WBS 1.2 assumes responsibility for achieving and maintaining the LAr in the appropriate state for the experiment.

The systems covered under WBS 1.2 perform three major tasks.

- Supply cooling to the cryostat using stored liquid nitrogen (LN2) as the refrigerant.

- Purify the liquid argon as received to a contaminant level where the drift lifetime of the signal electrons exceeds $3 \mathrm{~ms}$, twice the maximum drift-time.

- Continuously purify the liquid to contend with out-gassing from the vessel wall and cables.

In practice the same equipment used to clean the argon as-received serves to perform the continuous purification. Table 6.1 summarizes the specific requirements for the cryogenic and purification systems.

The systems proposed will meet these requirements with a straightforward design using components commonly in use in low temperature systems everywhere. Reliability comes from using redundant elements and stored LN2 for refrigeration. The MicroBooNE cryogenics design has been informed by the experiences of the ICARUS experiment, ArgoNeuT, and the Fermilab Liquid Argon Purity Demonstration (LAPD). 


\begin{tabular}{|c|c|c|}
\hline Parameter & Value & Motivation \\
\hline Argon purity & $<100$ ppt $\mathrm{O}_{2}$ equivalent & $\begin{array}{l}\text { Need to identify a minimum ionizing } \\
\text { particle at the longest drift distance }\end{array}$ \\
\hline Argon purity & $<2 \mathrm{ppm} \mathrm{N}_{2}$ & Need to preserve scintillation light output ${ }^{1}$ \\
\hline Liquid argon receiving rate & One tank truck per day & Practical rate including testing of argon \\
\hline LAr temperature spread & $<1^{\circ} \mathrm{K}$ & Maintain uniform electron drift velocity. \\
\hline Pressure stability & $<2$ PSI during operation & Maintain stable argon conditions \\
\hline $\begin{array}{l}\text { Liquid argon recirculation } \\
\text { rate }\end{array}$ & 1 volume change/day & $\begin{array}{l}\text { Ensure that adequate purity can be } \\
\text { maintained after filling the cryostat }\end{array}$ \\
\hline Backup controls power & 1 hour & To allow startup of gas-powered generator \\
\hline Cryostat insulation & $<15 \mathrm{~W} / \mathrm{m}^{2}$ & $\begin{array}{l}\text { Avoid excessive convection currents and } \\
\text { avoid bubble formation }\end{array}$ \\
\hline Cryogenic Capacity & $10 \mathrm{kWatts}$ & Heat load of electronics plus heat leak \\
\hline $\begin{array}{l}\text { Cryostat Max Allowable } \\
\text { Working Pressure }\end{array}$ & $30 \mathrm{psig} /$ full vacuum & Relief sizing/ability to evacuate \\
\hline Seismic Load on Equipment & $0.1 \mathrm{~g}$ & Uniform Building Code Zone 1 \\
\hline
\end{tabular}

Table 6.1 Cryogenic and purification system requirements.

The WBS 1.2 MicroBooNE cryogenics task divides into six major sub-tasks. The first sub-task documents the effort of the cryogenics team and provides a safety document used by the laboratory in reviewing the system before granting an operations permit. The other five subtasks provide the actual design and specify the procurement of each piece of equipment.

The cryogenics system sets up the equipment and defines the method to receive liquid argon (LAr) from a commercial vendor, test its purity, and further purify it. Purchased LN2 stored in a Dewar supplies the refrigeration for the LAr. Cryogenic pumps and filter vessels purify the liquid in the detector. A special compressor and heat exchanger cool down and purge the vessel from air to argon at a controlled cool-down rate. Finally, cryogenic controls monitor and regulate the state of the argon in the detector and provide information on the state of the argon to other parts of the detector such as the TPC and the PMT systems. Specially constructed purity monitors measure the electron drift lifetime, and commercial analytic equipment characterizes the nitrogen, oxygen, and water contaminant levels in the argon

\subsection{Preliminary Design}

Figure 6.1 and Figure 6.2, derived from Fermilab drawings 3974.200-ME-466281, sheets 1 and 2 , show the general design of the cryogenic system. It includes a section for receiving commercial argon in trucks, a section for purifying the argon, a system for condensing the boiloff argon, and systems for circulating gaseous and liquid argon. Particular attention has been given to avoid direct condensation of any of the gaseous argon in the cryostat ullage directly back into the liquid since this warm gaseous argon tends to contain a high concentration of contaminants, particularly water. ${ }^{2}$ 


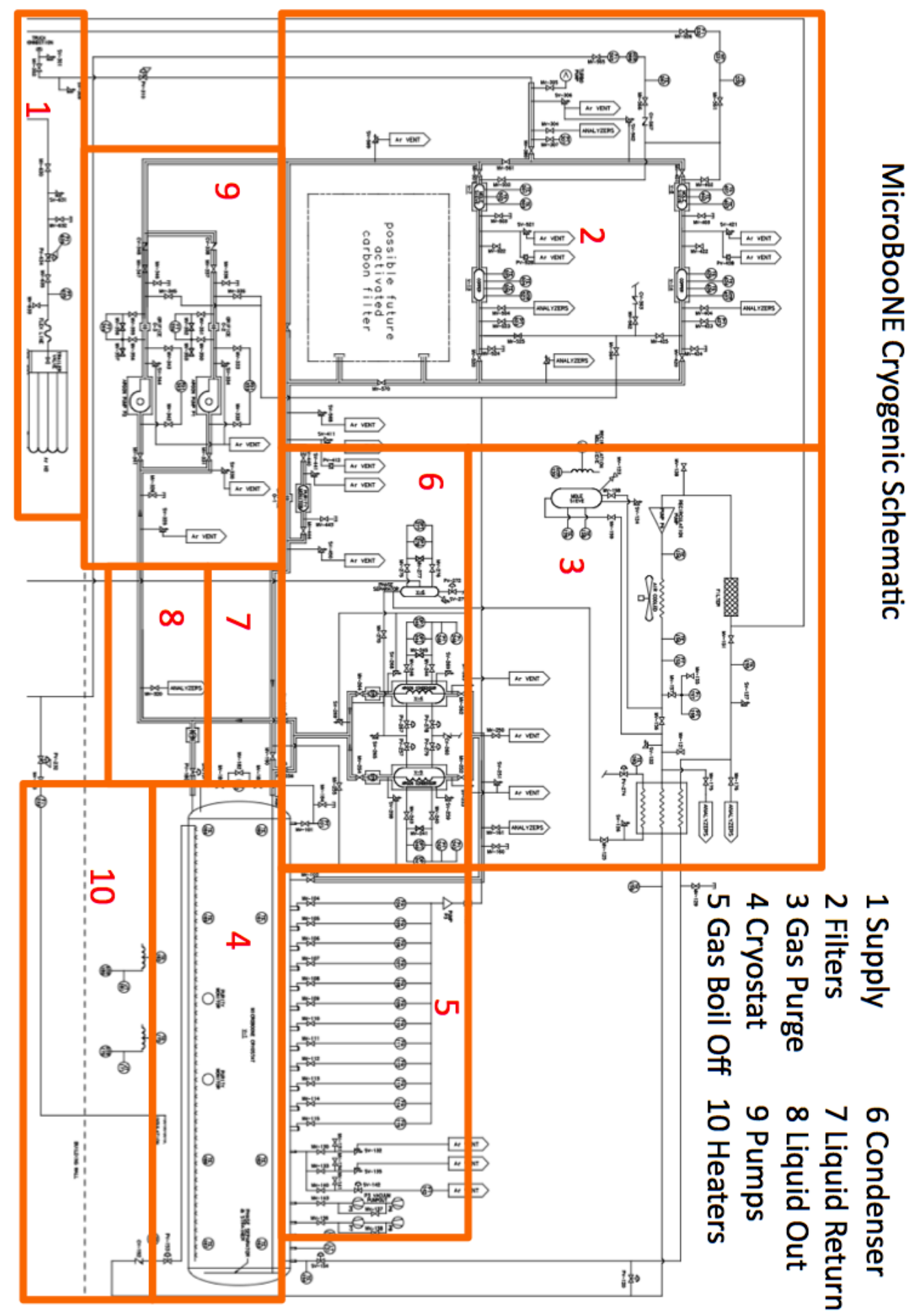

Figure 6.1 Sheet 1 of the piping and instrumentation design (P\&ID) showing the cryostat, main filter loop, cool-down system and refrigeration circuit. 


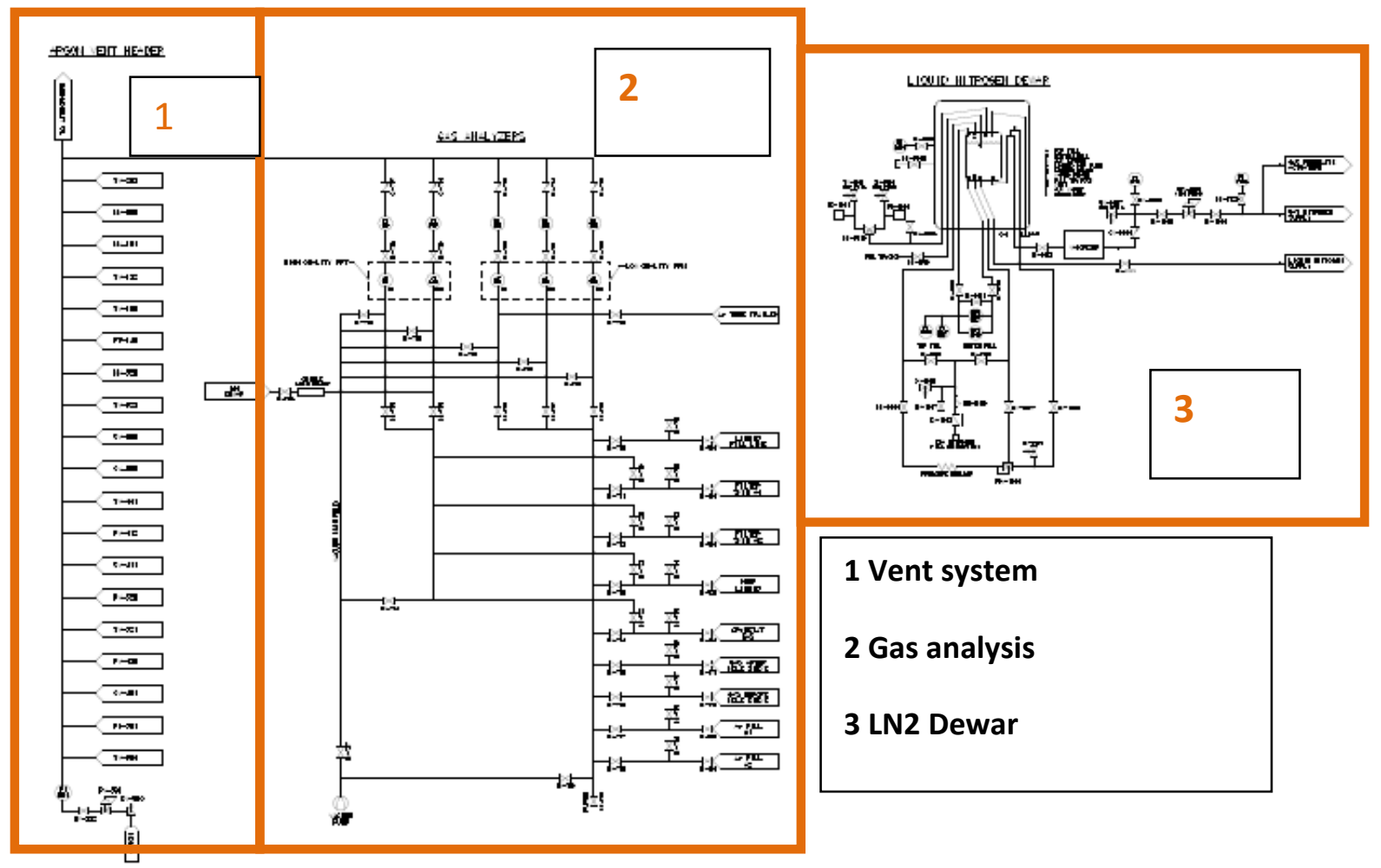

Figure 6.2 Sheet 2 of the P\&ID shows the gas analysis system and the LN2 Dewar.

\subsubsection{Argon Receiving}

A vendor truck delivering LAr hooks up to the truck connection shown in Figure 6.1. The line connects to a vacuum pump to allow purging from air to argon and to a gas analyzer connection to allow the truck contents to be checked against the specifications for purchased LAr. Upon acceptance of the truck contents, the piping changes to send the LAr through one of the filtration skids before entering the cryostat vessel. A truck holds about 3500 gallons of LAr, and the cryostat has a volume of approximately 38,000 gallons. It thus takes ten truckloads to fill the tank and leave adequate ullage space. Each truckload will be tested for purity before being accepted using the commercial analysis equipment included in the project cryogenic controls.

\subsubsection{LN2 System}

Purchased LN2 provides refrigeration for the experiment. After the shutdown of the Tevatron, an 11,000 gallon Dewar from the Fermilab Central Helium Liquefier will become available for use in MicroBooNE. The experiment should use about 60 gallons of liquid nitrogen per hour in normal operations. More refrigeration can be provided for the initial cool-down and fill period by simply supplying more LN2. To reduce installation cost, foam insulation surrounds the LN2 piping, rather than vacuum jackets. Foam insulated copper pipe is easy to install and has been in 
use at Fermilab for years on different experiments, notably for the Chicago Cyclotron magnet in E665, and more recently LAPD.

\subsubsection{Argon Purification}

The argon purification system operates in several different modes. The system can purify liquid argon as it is received from the delivery truck and deliver it directly into the cryostat. It can provide continuous purification of the cryostat fill. It can also perform both functions at the same time. Dual liquid argon pumps and dual filter skids are installed, and filter skids can be regenerated independently.

\subsubsection{Liquid Argon Pumps}

The MicroBooNE design specifies Barber-Nichols BNCP-32B centrifugal pumps. These 10-12 gal/min capacity pumps have been used at CERN for many years, and they currently operate in the Fermilab Liquid Argon Purity Demonstrator (LAPD). Magnetic coupling prevents contamination through shaft seals. Their flow capability with two pumps allows recycling the liquid argon in the vessel approximately once per day. Variable frequency drives allow the flow of the pumps to be adjusted if required. Normal operation entails both pumps running simultaneously. The pumps mount on a single skid, shown in Figure 6.3 , that can be prefabricated and installed in the enclosure as a unit. Either pump may be valved out of the stream and disassembled for maintenance while the other continues to run. A third pump will be purchased as a non-installed spare.

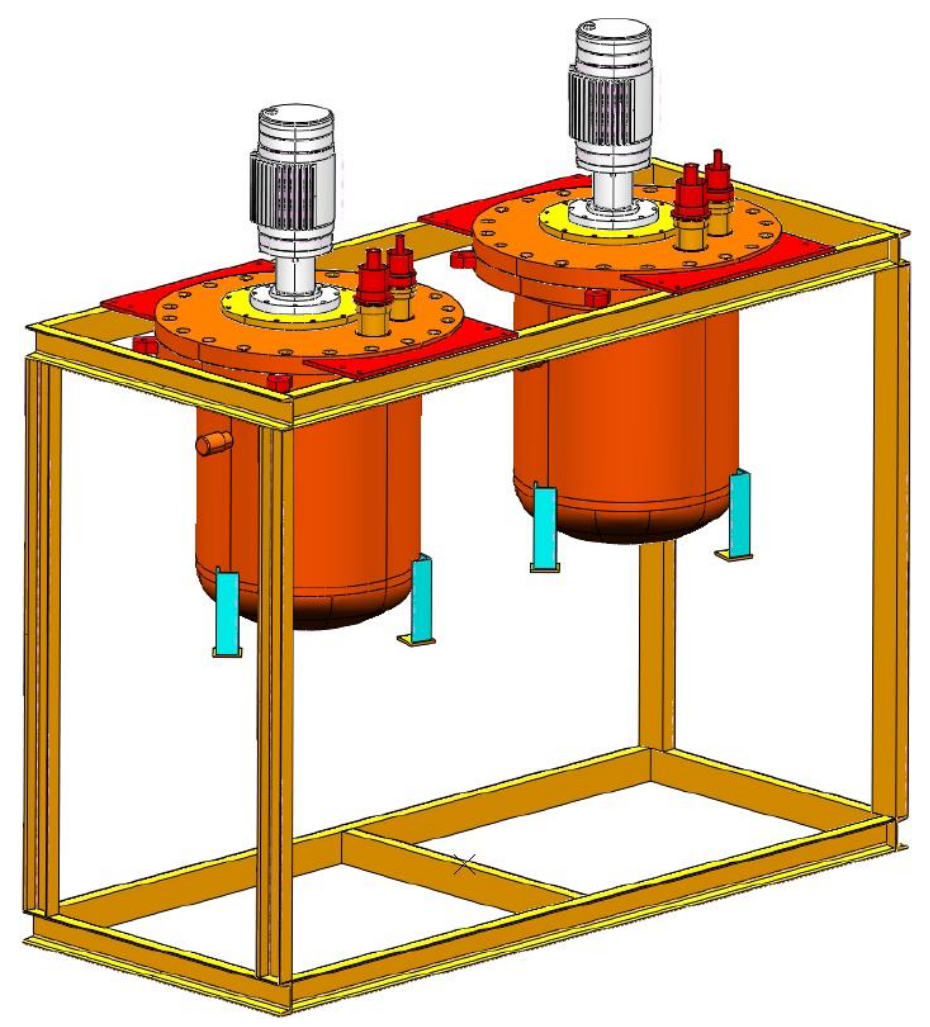

Figure 6.3 LAr pump skid. 


\subsubsection{Filter system}

Dual filter systems, shown in Figure 6.4, provide purification of the LAr for MicroBooNE. Each filter system consists of a bed of molecular sieve to remove moisture and another bed of activated copper material to remove oxygen. Either can be taken off line and regenerated while the other continues to run. A warm line is available to reactivate the copper bed in place with a mixture of gaseous argon and hydrogen. Likewise, external heaters and gaseous argon can reactivate the molecular sieve without any need for disassembly.

The molecular sieve bed contains 118 lbs of 208604-5KG Type 4A, Molecular Sieve material from Sigma-Aldrich Company. The oxygen filter bed contains 132 lbs of CU-0226 S 14 X 28 Oxygen Reactant from Research Catalysts, Inc. In case the materials have to be replaced, the filter vessels allow for a replacement of the materials without having to cut the vessels open.

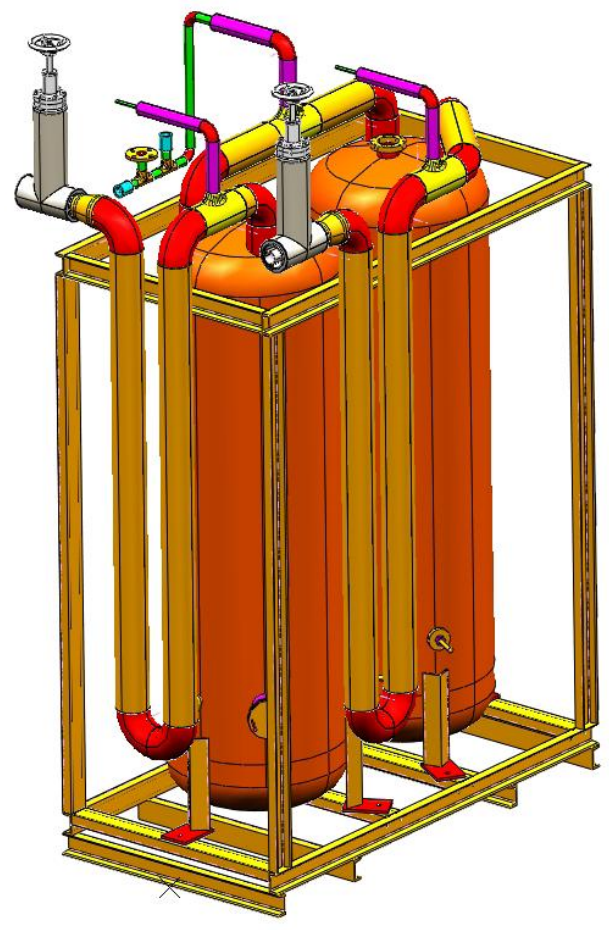

Figure 6.4 A MicroBooNE filter skid with a Molecular Sieve bed and Oxygen filter bed.

\subsubsection{Cryostat Filling}

The filling procedure starts a warm cryostat filled with air and finishes with a cold cryostat filled with LAr at a low contamination of water and oxygen. Two methods are provided to remove the original atmosphere: displacement of the air by argon gas, and vacuum purging. Proving the viability of the displacement method, with an eye towards the $20 \mathrm{kton}$ TPC proposed for LBNE, would satisfy one of MicroBooNE's main R\&D goals. The displacement procedure followed by introduction of cold gas to control temperature differentials between the various components of 
the detector nominally serves as the normal method for removing the air and cooling down the cryostat.

\subsubsection{Gas Purge \& Recirculation system}

Piping at the bottom of the cryostat allows the heavier argon gas to act as a "piston" to force all of the air out the top vent. Between five and ten volume changes will be used during the purge process.

After the initial purge, a compressor recirculates the gas, and a heat exchanger allows the vessel to be cooled down at a controlled rate to prevent damage to the TPC caused by differential contraction between its frame and wires. Water constitutes the main contaminant of concern at this stage, and the compressor circuit operates with a molecular sieve bed that will remove water vapor from the vessel before cool down begins. Surface mount heaters allow warming the cryostat to aid in the transport of water from the vessel surface.

The purge method was tested successfully at Fermilab with both a 162 cubic feet vessel $^{3}$ and the 785 cubic feet LAPD cryostat. The LAPD experience showed that the concentration of water and oxygen in the vessel after nine volume exchanges met the allowed specification for the delivered LAr. The LAPD system also demonstrated that surface mounted heaters can be used to drive water off of the vessel.

The LAPD system successfully achieved a $3 \mathrm{~ms}$ electron lifetime in the LAr. This electron lifetime corresponds to a contamination level of $100 \mathrm{ppt}$ oxygen equivalent. Based on these results, the MicroBooNE system should meet the specifications for purity.

\subsubsection{Vacuum purge system}

A backup pumping system provides the capability of evacuating the cryostat in the event of problems with the purge method. This consists of a pair of turbo molecular pumps (recycled from experiment E835). Scroll pumps used elsewhere in the system during normal operations (for tasks such as to regenerating the filters) serve as forepumps during evacuation.

The availability of these turbo molecular pumps and comparisons with the pumping system used at ICARUS ${ }^{4}$ determined the sizing of the system.

\subsubsection{Cryostat Controls and Monitors}

The proposed control system utilizes a commercial Programmable Logic Controller (PLC). Its design follows others built by the Fermilab cryogenics group recently, including a system for the helium plant in the New Muon Lab for superconducting RF cavity testing, ArgoNeuT, LAPD, and one under way now for the Minerva liquid helium target.

\subsubsection{Controls system and instruments}

The control system uses a Siemens S7-400 controller. The PLC provides for several loops to adjust the temperature rate at cool down, and to set the temperature for the condenser operations that control the temperature of the liquid argon in the vessel. The controller logs data from 
instruments for pressures, temperature, differential pressures, and liquid levels; and it makes the data available to operators. It provides remote readout of the status of the oxygen deficiency hazard $(\mathrm{ODH})$ monitors. IFix, a standard commercial product used at Fermilab, serves as the human-machine interface. Operators can monitor and adjust cryogenic controls both locally and remotely through secure connections.

\subsubsection{Purity monitors}

Purity monitors test the purity of the liquid in the cryostat and to monitor the effectiveness of the filter system. These purity monitors (shown in Figure 6.5) have been in use on the test systems at the Fermilab Proton Assembly Building for years. They are based on the design used in ICARUS. The monitor consists of a drift chamber with a photocathode at one end and an anode at the other. A xenon flash lamp pulse liberates electrons from the cathode, and these electrons drift under an electric field to the anode. The electron drift lifetime in the liquid can be extracted by measuring the drift time of the electrons from the cathode to the anode and the ratio of the charge arriving at the anode compared to the charge leaving the cathode. Two purity monitors mount inside the cryostat; and a third installs just downstream of the filters. The two in the vessel always remain "on line". The one in the liquid return line operates in a vessel that is filled with liquid and then isolated when taking a reading.

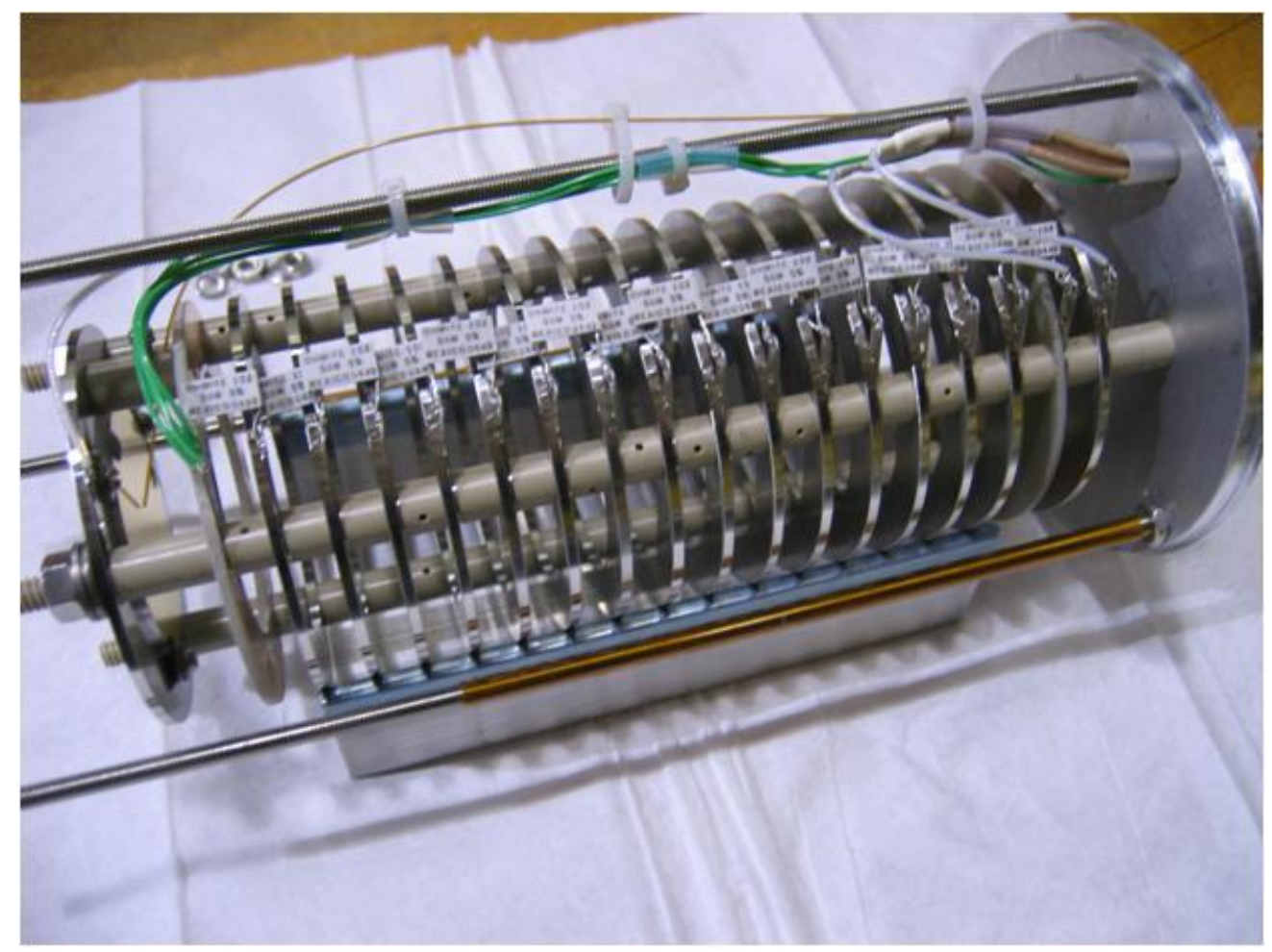

Figure 6.5 Purity monitor. 
${ }^{1} \mathrm{~A}$ system to test the effects of materials on the electron drift lifetime in liquid argon and observations

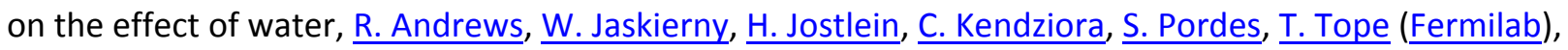
FERMILAB-PUB-09-355-E. Jul 2009. 16 pp. Published in Nucl.Instrum.Meth. A608 (2009) 251-25.

${ }^{2}$ Oxygen contamination in liquid Argon: Combined effects on ionization electron charge and scintillation light. WArP Collaboration (R. Acciarri (L'Aquila U. \& INFN, Aquila) et al.). Apr 2008. 16 pp.

Published in JINST 5 (2010) P05003

${ }^{3}$ W. Jaskierny, H. Jostlein, S. Pordes, P.A. Rapidis, T. Tope, "Test of Purging a small tank with Argon", October 28, 2006, LArTPC DocDB \# 223.

${ }^{4}$ J. Howell, "MicroBooNE cryostat evacuation option costs and notes, April 19, 2009. MicroBooNE DocDB \#1053. 


\section{Cryostat (WBS 1.3)}

\subsection{Overview}

Three major subsystems make up the MicroBooNE neutrino detector cryostat: a stainless steel (type 304) vessel to contain the liquid argon (LAr) and all the active detector elements, front and rear supports to carry the weight of the fully loaded cryostat, and foam insulation covering the cryostat outer surfaces to reduce heat input from the ambient to a sufficiently low level to prevent large temperature gradients and boiling of the LAr. Two technical factors drive the specific design of this cryostat and the cryogenic systems for the MicroBooNE detector: the very high purity LAr needed to allow ionization electrons to drift to the sense wires with low probability of capture, and the high degree of thermal homogeneity needed to avoid the introduction of non-constant drift velocities for the ionization electrons.

Ionization electrons must drift up to $2.5 \mathrm{~m}$ across the active without significant attenuation by attachment to electronegative contaminants in the LAr. This dictates that the argon be kept free of electronegative contaminants to the level of 100 parts-per-trillion (ppt). The cryostat must be designed to minimize outgassing (desorption) and to avoid leakage and diffusion of air into the system. This imposes strict quality assurance demands on all welds for penetrations into the cryostat and on cleaning and handling procedures for the finished vessel. Stringent cleanliness requirements must also be enforced for all components that install inside the cryostat. Finally achieving the required level of purity requires a powerful and effective purification facility, described in Chapter 6, to remove electronegative contaminants from the argon in the initial fill and introduced over time by leaks and outgassing of system components.

The electron drift velocity $\left(\mathrm{V}_{\mathrm{d}}=1600 \mathrm{~m} / \mathrm{s}\right.$ with a LAr temperature dependence $\Delta \mathrm{V}_{\mathrm{d}} / \mathrm{V}_{\mathrm{d}}=$ $-0.019 \Delta \mathrm{T}$ ) must remain constant in magnitude and direction throughout the active LAr volume to avoid distortion of the mapping of drift time into the $\mathrm{X}$-coordinate along the drift direction. This requirement limits the allowable temperature variations of the LAr over significant volumes of the TPC in the cryostat to much less than $0.1 \mathrm{~K}$ and the laminar and turbulent flow rate of LAr to less than $1 \mathrm{~m} / \mathrm{s}$. These requirements limit fractional errors in velocity, and therefore in $\mathrm{X}$ coordinate determination, to be less than $0.1 \%$. The constraints on constancy of drift velocity affect the design by imposing limits on the acceptable heat flux through the insulation.

Table 7.1 summarizes the specific cryostat requirements established from the experiment constraints discussed above. The MicroBooNE Brookhaven National Laboratory (BNL) group leads the design effort to satisfy these requirements. The BNL group has extensive experience in similar efforts associated with the ATLAS LAr calorimeter. The actual MicroBooNE cryostat will be fabricated by an external vendor under the supervision of BNL. 


\begin{tabular}{|l|l|l|}
\hline $\begin{array}{l}\text { Cryostat head } \\
\text { Removal }\end{array}$ & At least once during lifetime & $\begin{array}{l}\text { Allow for repair or replacement of } \\
\text { detector components }\end{array}$ \\
\hline Cryostat insulation & $<15 \mathrm{~W} / \mathrm{m}^{2}$ & $\begin{array}{l}\text { Eliminate the effect of convection } \\
\text { currents on the electron drift }\end{array}$ \\
\hline LAr bubble formation & No detectable rate. & $\begin{array}{l}\text { Eliminate the effect of convection } \\
\text { currents on the electron drift }\end{array}$ \\
\hline $\begin{array}{l}\text { Cryostat maximum } \\
\text { allowable working } \\
\text { pressure (MAWP) }\end{array}$ & 30 psig/full vacuum & $\begin{array}{l}\text { Allows use of reasonably-sized code- } \\
\text { stamped relief valves. }\end{array}$ \\
\hline Seismic load & UBC Zone 1, acceleration $<0.1 \mathrm{~g}$ & Maximum seismic event at Fermilab. \\
\hline
\end{tabular}

Table 7.1 Parameters for the cryostat design.

\subsubsection{Cryostat Mechanical Design Considerations}

The cryostat contains the LAr, the active detector elements, consisting of the TPC and photomultiplier tube (PMT) array, and sensors required to monitor pressure, level, temperature, and purity of the LAr. It must be constructed to the latest ASME boiler code requirements and stamped to indicate that it meets the code. The cryostat vessel features single-walled construction cylindrical in shape with domed caps closing each end as shown in Figure 7.1. One end can be removed for detector installation and modification. The cryostat outer diameter reaches the maximum standard size for over-the-road transport and will require special planning for delivery to Fermilab.

The vessel surface has 34 nozzle penetrations for cryogenic and electrical services. Figure 7.1, Figure 7.2 , and Table 7.2 summarize the properties of these penetrations, giving the tube and flange dimensions. When filled with LAr during operations, a liquid nitrogen (LN2) cooling system maintains the operational conditions and absorbs heat leaking from the warm outside. The cryogenic system described in Chapter 6 provides and controls this refrigeration, as well as the purity, temperature, pressure, and volume of the LAr in the cryostat.

Two sets of penetrations in the cryostat vessel, shown in Figure 7.1, provide for the removal of air in the cryostat before filling with purified LAr. This process involves establishing a flow of argon gas to displace and flush the air from the cryostat volume. The continued flow of argon gas also further dilutes oxygen and water contamination in the vessel. A perforated pipe along the bottom of the vessel supplies the argon gas, and a similar pipe at the top of the vessel allows for gas removal. The system of piping also serves to cool the contents of the vessel before filling 
with LAr. The cooling rate will be controlled to avoid excessive differential thermal contraction of the detector elements.

To accommodate the unlikely, but conceivable, outcome that this displacement and dilution of air fails to produce adequate initial LAr purity, two large cross section ports at the top of the vessel allow for evacuation of the cryostat with mechanical pumps. Operational responsibility for the fill and purge or vacuum purge procedures fall within the domain of the cryogenic system described in detail in Chapter 6.

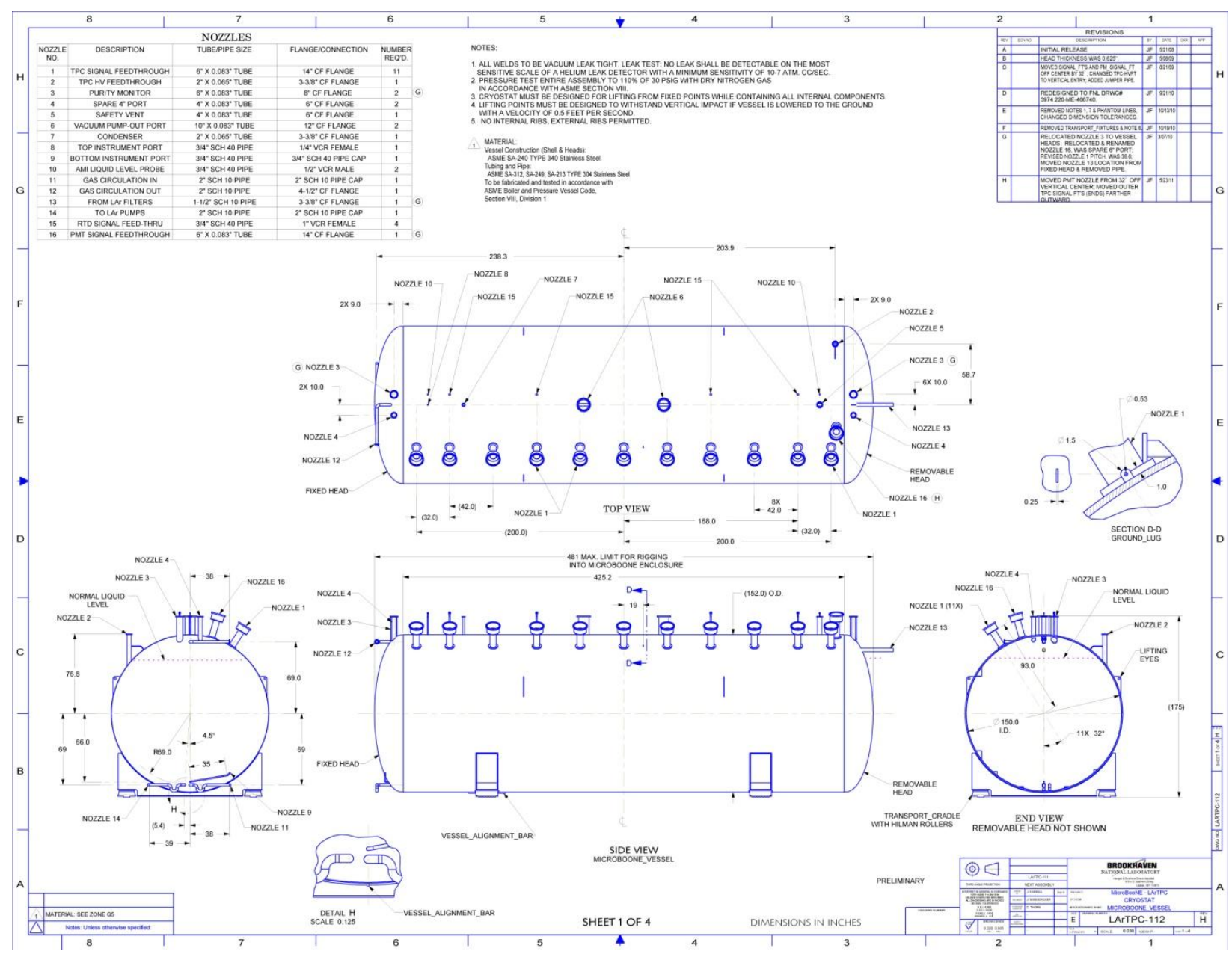

Figure 7.1 Views of the cryostat vessel from the side, top and ends.

\subsubsection{Achieving Acceptably Low Heat Leak}

Ionization electrons must drift up to $2.5 \mathrm{~m}$ across the active region without significant attenuation by attachment to electronegative contaminants in the LAr. This dictates that the argon be kept free of electronegative contaminants to the level of 100 parts-per-trillion (ppt). The cryostat must be designed to minimize outgassing (desorption) and to avoid leakage and diffusion of air into the system. This imposes strict quality assurance demands on cleaning and handling procedures for the finished vessel and on leakage rates of all welds for penetrations into the cryostat. Stringent cleanliness requirements must also be enforced for all components 
that are installed inside the cryostat. Finally, achieving the required level of purity requires a powerful and effective purification facility, described in Chapter 6, to remove electronegative contaminants from the argon in the initial fill and introduced over time by leaks and outgassing of system components.

The requirement of constant drift velocity means that the LAr must remain free of boiling caused by high heat leaks. Boiling agitates the LAr, and this motion will distort the electron drift path and may cause the TPC sensor wires to move. Temperature differences of the LAr within the TPC volume must also be limited since the electron drift velocity depends strongly on temperature $(-1.9 \% / \mathrm{K}$, see MicroBooNE DocDB 412), and since temperature differences will drive convection of the LAr which would cause deviation of the drift trajectories from the desired straight lines in the constant electric field. Limiting heat influx into the cryostat by adequate insulation of the surface prevents these effects from disturbing the proper functioning of the TPC.

\subsubsection{Access, Internal Support, and Interface Considerations}

Physics requirements, mechanical design, and cost determine the construction specifications for the internal width and length of the cryostat. As stated above, the vessel has provisions to enable one of its ends to be opened for installation of the detector systems. Extensive discussions have already been held to establish piping and wiring lists and to define the details of this work. Brookhaven engineers have designed tracks, rails, and hangers for the insertion and anchoring of the TPC and PMT support racks. The design group has also established the size and location of the eleven hermetic pin carrier penetrations placed near preamplifiers located on top of the TPC. These locations reduce the length of wiring carrying data signals out through the feed through nozzle, keeping the volume of cabling, and therefore outgassing, as low as possible. Other wiring used for TPC and PMT power, high voltages, instrumentation, and for temperature, pressure and level sensing will be strung along predetermined paths and supported by hangers to each of their assigned feedthroughs. Purity monitors have their own feedthroughs with special hermetic connectors needed to accommodate their high voltage, fiber optic, and general instrument wiring. Refer to Table 7.2 for a listing of the nozzles for these feedthroughs.

Shielding and grounding in large detectors must be carefully managed to minimize electronic noise in circuitry and wiring. To avoid ground loops, the cryostat vessel will be grounded in only one place, allowing it to act as a Faraday cage. Special rules for wiring, shielding, and prevention of ground loops will be stressed throughout the installation process. The individual active detector, electronics, and installation chapters (Chapters 8-11) give further details.

\subsubsection{Detector Platform Stability}

All cryogenic systems designs must include provisions for the control of stresses introduced as the components cool from room temperature to the low operating temperature. The change from warm to cold causes shrinkage of parts, adding additional stresses to unknown locked-in stresses. These can result in unpredictable changes in stresses that could cause unacceptable twisting and 
bending. In very large structures, like the ATLAS detector, annealing of parts in an oven after fabrication removes these strains.

Smaller, thin walled vessels like the MicroBooNE cryostat usually experience less severe forms of these effects; but for MicroBooNE an additional consideration enters through the dramatic increase in the weight of the vessel and its contents as it fills with LAr, from $33.5 \mathrm{t}$ (metric tons) empty to 203 t full. Vessel walls deflect, and the PU foam supports (described later) compress, changing relative positioning in the direction transverse to the vessel axis. The axial length of the vessel shrinks 1.5 inches from room temperature to $90 \mathrm{~K}$. The design process uses finite element ANSYS stress-strain analyses to adjust the warm cryostat parameters to achieve desired conditions during LAr operations. These calculations have been made for the vessel drawn in Figure 7.1, which has a 1.0 inch thick cylindrical wall.

\begin{tabular}{|c|c|c|c|c|}
\hline $\begin{array}{l}\text { Nozzle } \\
\#\end{array}$ & Description & Tube/pipe size & Flange/Connection & Quantity \\
\hline 1 & $\begin{array}{l}\text { TPC Signal } \\
\text { Feedthrough }\end{array}$ & 6" X 0.083" Tube & 14" Cf Flange & 11 \\
\hline 2 & TPC HV Feedthrough & 6" X $0.065 "$ Tube & 8" Cf Flange & 1 \\
\hline 3 & Purity Monitor & 6" X 0.083" Tube & 8" Cf Flange & 2 \\
\hline 4 & Spare 4" Port & 4" X 0.083" Tube & 6" Cf Flange & 2 \\
\hline 5 & Safety Vent-Tandem & 4" X 0.083" Tube & 6" Cf Flange & 1 \\
\hline 6 & $\begin{array}{l}\text { Vacuum Pump-Out } \\
\text { Port }\end{array}$ & $\begin{array}{l}10 " \text { X } 0.083 " \\
\text { Tube }\end{array}$ & 12" Cf Flange & 2 \\
\hline 7 & Condenser & 2" X 0.065" Tube & 3-3/8" Cf Flange & 1 \\
\hline 8 & Top Instrument Port & 3/4" Sch 40 Pipe & 1/4" Vcr Female & 1 \\
\hline 9 & $\begin{array}{l}\text { Bottom Instrument } \\
\text { Port }\end{array}$ & 3/4" Sch 40 Pipe & $\begin{array}{l}\text { 3/4" Sch } 40 \text { Pipe } \\
\text { Cap }\end{array}$ & 1 \\
\hline 10 & $\begin{array}{l}\text { AMI Liquid Level } \\
\text { Probe }\end{array}$ & 3/4" Sch 40 Pipe & 1/2" Vcr Male & 2 \\
\hline 11 & Gas Circulation In & 2" Sch 10 Pipe & 2" Sch 10 Pipe Cap & 1 \\
\hline 12 & Gas Circulation Out & 2" Sch 10 Pipe & 4-1/2" Cf Flange & 1 \\
\hline 13 & From LAr Filters & $\begin{array}{l}1-1 / 2 " \text { Sch } 10 \\
\text { Pipe }\end{array}$ & 3-3/8" Cf Flange & 1 \\
\hline 14 & To LAr Pumps & 2" Sch 10 Pipe & 2" Sch 10 Pipe Cap & 1 \\
\hline 15 & $\begin{array}{l}\text { RTD Signal } \\
\text { Feedthrough }\end{array}$ & 3/4" Sch 40 Pipe & 1" Vcr Female & 4 \\
\hline 16 & $\begin{array}{l}\text { PMT Signal } \\
\text { Feedthrough }\end{array}$ & 6" X 0.083" Tube & 14" Cf Flange & 1 \\
\hline
\end{tabular}

Table 7.2 List of nozzle penetrations in vessel (refer to Figure 7.2 for locations).

In market surveys conducted to establish cryostat costs, some vendors have suggested constructing the vessel with a thinner wall and adding external stiffening "ribs" to meet the requirement for an evacuable vessel at a reduced cost. Since an apparent, though modest, cost reduction follows from these designs, the final solicitation of bids for the vessel allows vendors 
to opt for a "ribbed" design. To avoid inflated bids, vendors will not be required to perform their own finite element analysis of their designs, and hence they will almost certainly choose to meet code requirements by using standard design rules. Therefore, upon receiving final drawings from the selected vendor, the BNL team will repeat the ANSYS analysis to verify acceptable deflections from room to cryogenic temperatures. This TDR presents results of calculations obtained for a 1.0 inch thick wall with no "ribs"; the values for the vessel as finally designed may differ.

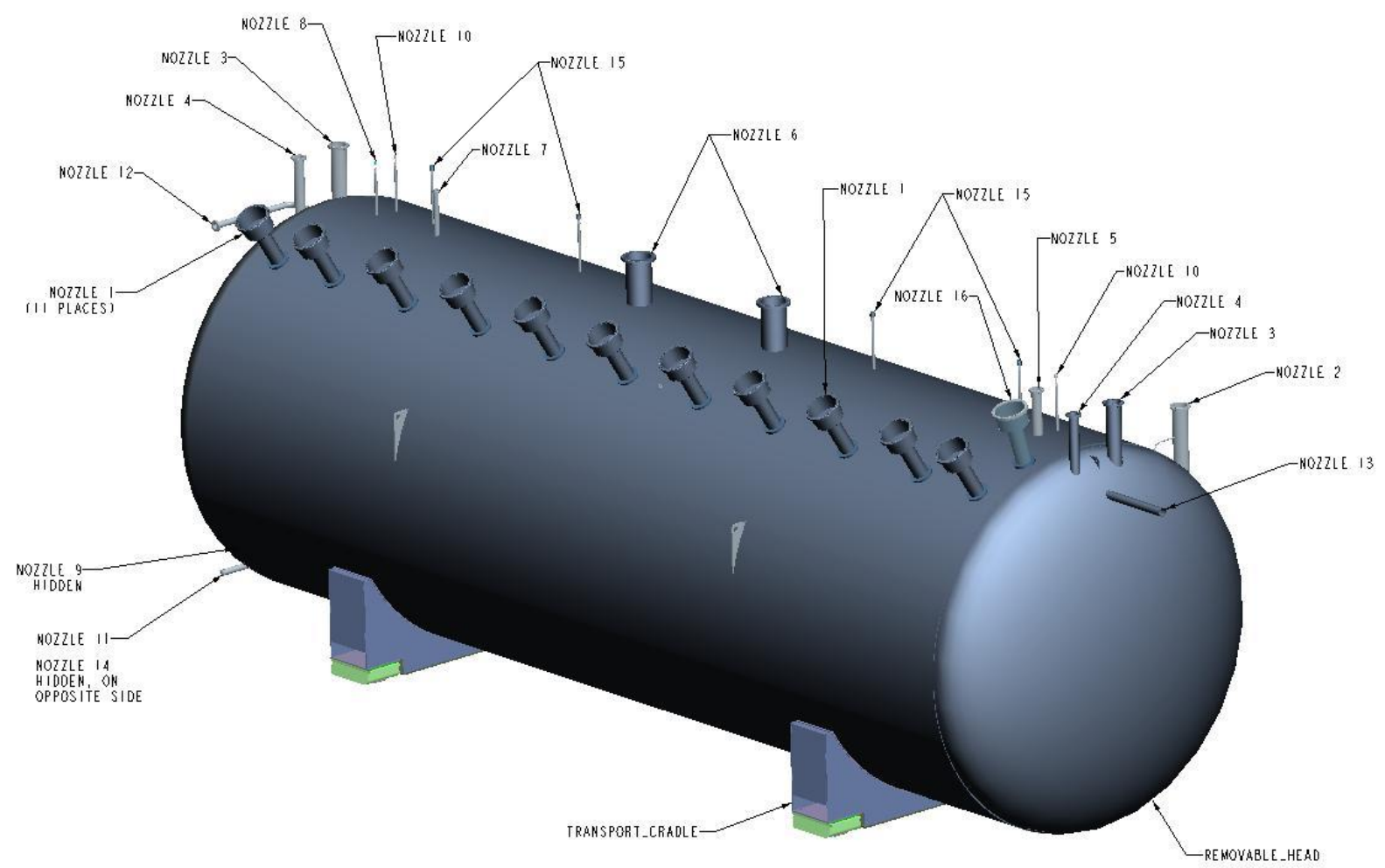

Figure 7.2 Isometric view of the vessel, with the nozzles labeled to correspond to Table 7.2. The vessel is shown mounted on temporary saddles that will be provided by the vendor for transport and insertion of the active detectors in the assembly area. Refer to Chapter 11 for installation details.

Two high-density PU cradles support the MicroBooNE vessel. Extensive ANSYS modeling shows that both the fully loaded vessel and foam supports remain lightly stressed. Maximum fully loaded vessel equivalent stress reaches $14 \mathrm{kpsi}$ (97 MPa), and the support stress maximum falls below $64 \mathrm{psi}(0.4 \mathrm{MPa})$, a value chosen to be very low to minimize creep. Interestingly, filling the vessel with LAr causes the PU saddles to compress and the vessel to move down 0.100 inch $(2.5 \mathrm{~mm})$; but the TCP detector rails remain flat to within 0.040 in $(1.0 \mathrm{~mm})$. The fully loaded vessel deflects by a maximum 0.080 in $(2.0 \mathrm{~mm})$ at the bottom and sags by 0.240 in $(6.0$ $\mathrm{mm})$ at the top. 
Both hand calculations and ANSYS modeling verify the ability of the MicroBooNE cryostat vessel supported by two PU foam saddles to withstand $0.1 \mathrm{~g}$ acceleration, equivalent to a magnitude- 6 earthquake on the Richter scale. This satisfies a common code requirement. All calculations and modeling were done by converting a $0.1 \times \mathrm{g}$ acceleration to 44,700 pounds equivalent force on the cryostat, with the force applied to the cryostat vessel in both axial and lateral directions. The calculations also show that deflections of the type that could cause toppling remained negligibly small.

With the force axially applied to the fully loaded vessel with two saddle supports sharing the acceleration load, the foam deformation equals 0.08 inches $(2 \mathrm{~mm})$ in shear, with a maximum stress of $52 \mathrm{psi}(0.36 \mathrm{MPa})$. The vessel deformation equals 0.26 inches $(6.4 \mathrm{~mm})$, with a maximum vessel stress $13.5 \mathrm{kpsi}(93 \mathrm{MPa})$. For the case where one saddle takes the total load these results must be multiplied by two.

With the force laterally applied over the length of the vessel, the foam deformation equals 0.19 inches $(4.7 \mathrm{~mm})$ in shear, with a maximum stress of $60 \mathrm{psi}(0.42 \mathrm{MPa})$. The vessel deformation equals 0.33 inches $(8.2 \mathrm{~mm})$ in the direction transverse to the beam, with a maximum stress 60 psi (0.42 MPa).

All deflections and stresses stay small and well within limits, and no risk exists for the vessel tipping over in a seismic event.

\subsection{The Cryostat Vessel}

The main body of the cryostat comprises a single walled cylinder with dished heads closing each end. The overall cryostat length spans 481 inches $(12.2 \mathrm{~m})$, and the dished heads each add approximately 28 inches $(0.71 \mathrm{~m})$. The vessel has an outer diameter of 152 inches $(3.86 \mathrm{~m})$ with a wall thickness of 1.00 inch $(2.54 \mathrm{~cm})$. It meets requirements to be pumped down to full vacuum. Safety reliefs will be set to 30 psig even though during normal operation the vessel will be at a pressure of about 3 psig. The cryostat will contain $170 \mathrm{t}$ of LAr, leaving an $8.9 \%$ ullage volume. The empty vessel weighs 67242 pounds $(33.5 \mathrm{t}$ ). When filled the total, including foam insulation and LAr, increases to 446699 pounds (203 t).

Two types of active detectors reside within the cold vessel, the time projection chamber (TPC) and the photomultiplier tube (PMT) array. Signal lines from the TPC and PMTs use most of the nozzle penetrations through the vessel. The remaining penetrations carry high voltage for the TPC, power for cold electronics, and power and signal information for monitoring LAr purity, liquid level, temperature and pressure. Refer to Table 7.2 for a listing of the penetrations.

\subsection{Cryostat Insulation}

A study of different types of insulation with the help of a computer program from the North American Insulation Manufacturers Association (NAIMA), and with the consideration of material and labor costs, led to the selection of 16 inches of spray-on PU foam as the preferred 
option. This choice meets heat loss requirements, mentioned above, to prevent disturbance of the electron trajectories in the TPC, and the reduced labor costs for installation of this form of insulation result in lower overall cost. The heat leak through the 16 inch thick layer of PU foam insulation is $11.4 \mathrm{~W} / \mathrm{m}^{2}$ as computed with the NAIMA code and $11.3 \mathrm{~W} / \mathrm{m}^{2}$ as computed in a simple cylindrical geometry for the cryostat from the manufacturer's data for the foam thermal conductivity as a function of temperature. This heat influx ensures that the outer surface temperature remains above the dew point for all contemplated conditions of temperature and humidity. The estimated total vessel insulation heat leak is $1600 \mathrm{~W}$. A total of $1200 \mathrm{~L}$ per day of liquid nitrogen refrigerates the cryostat and keeps operating parameters constant. This estimate excludes contributions from electronics, cryostat penetrations, and the cryogenic system (see also Chapter 6).

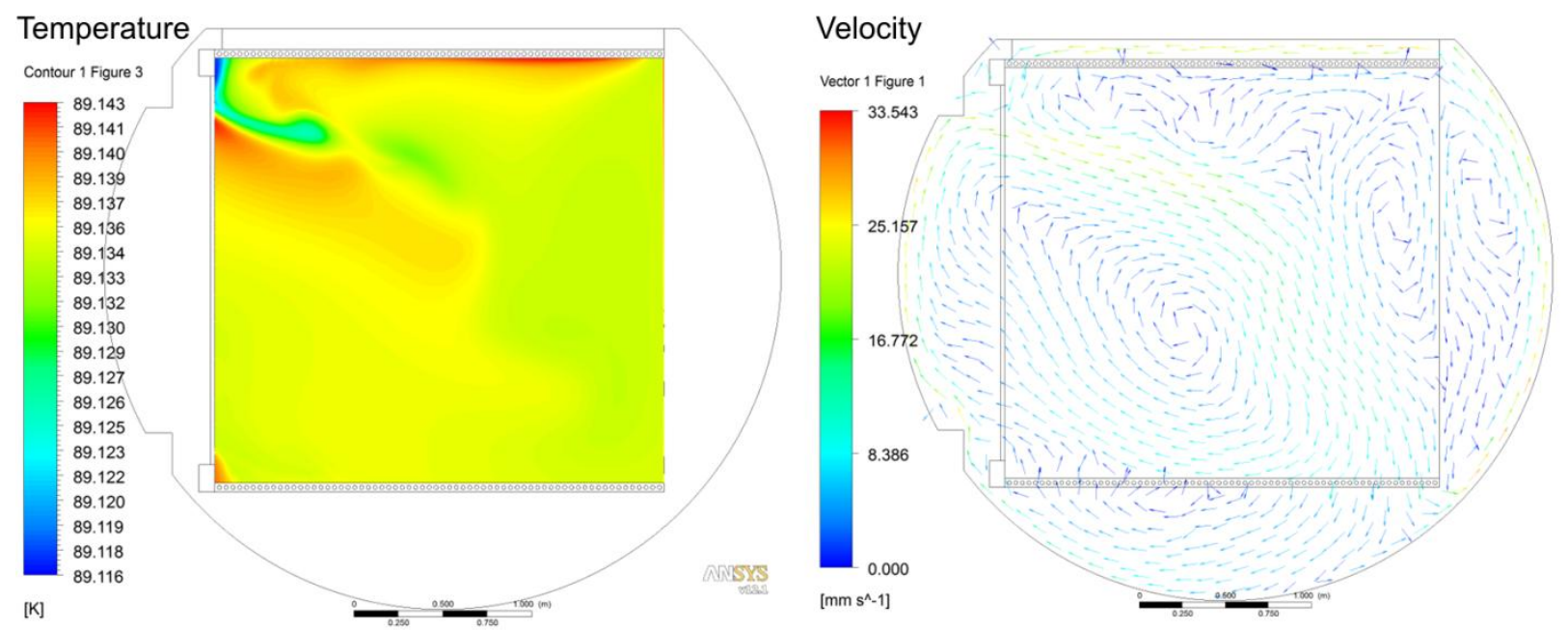

Figure 7.3 Temperature (left) and velocity (right) of the LAr at various points inside the vessel assuming a heat load of $13 \mathrm{~W} / \mathrm{m}^{2}$.

The temperature and velocity of the LAr in the cryostat has been modeled using a finite element analysis (ANSYS) assuming a heat load of $13 \mathrm{~W} / \mathrm{m}^{2}$. Figure 7.3 shows the results. Over the volume of the TPC, the maximum temperature spreads are less than $0.02 \mathrm{~K}$ and the LAr velocity variation is less than $2.5 \mathrm{~cm} / \mathrm{s}$. These maximum temperature and flow variations correspond to variations in the electron velocity of $0.04 \%$ and $0.002 \%$, respectively. These small potential perturbations of the electron trajectories indicate that the ability of the TPC to record charged particle trajectories will not be impaired, A number of calibrated temperature monitors will be distributed throughout the TPC volume to sample the thermal variation, but the insulation modeling indicates a large margin of safety even with the maximum allowable heat influx of 15 $\mathrm{W} / \mathrm{m}^{2}$ specified for the mechanical insulation..

\subsection{Cryostat Support}

The support system for the cryostat must provide mechanical stability through cool-down and filling of the vessel with $170 \mathrm{t}$ of LAr. A support structure built from PU foam satisfies this requirement. Liquid natural gas ships use large diameter (36") transfer lines insulated and supported by PU foam. While using foam for the cryostat support represents a significant 
extension of the methods used for lightly loaded 36" and smaller transfer lines, neither the degree of insulation provided by the foam nor the $203 \mathrm{t}$ total load poses problems for a design with two foam saddles. Accordingly, this option has been chosen for the support of the MicroBooNE cryostat.

Material with densities of $12-15$ pounds/ $\mathrm{ft}^{3}$ emerges as the ideal choice among the wide array of high density foams available for this application. This PU foam has a compressive strength maximum of nearly $500 \mathrm{psi}$ and a compressive modulus of 13,000 psi at room temperature. The cryostat's heavy loads can be managed by employing interface surfaces with sufficient area to reduce foam stresses to low values, and a conservative safety factor greater than 8 can be easily achieved. Further, due to the low modulus of the material, loads evenly distribute throughout the foam. This avoids highly stressed "hot spots" commonly seen in metal fabrications. The insulation factor is simply proportional to the thickness of the insulating material, and the saddles therefore provide better local insulation than the 16 inches of foam covering the remainder of the vessel. Figure 7.4 shows 120 degree foam saddles bonded in place on the exterior of the cryostat. The dimensions of a 120 degree foam saddle are 200 " wide by 66 " high by 36 " wide along the vessel axis.

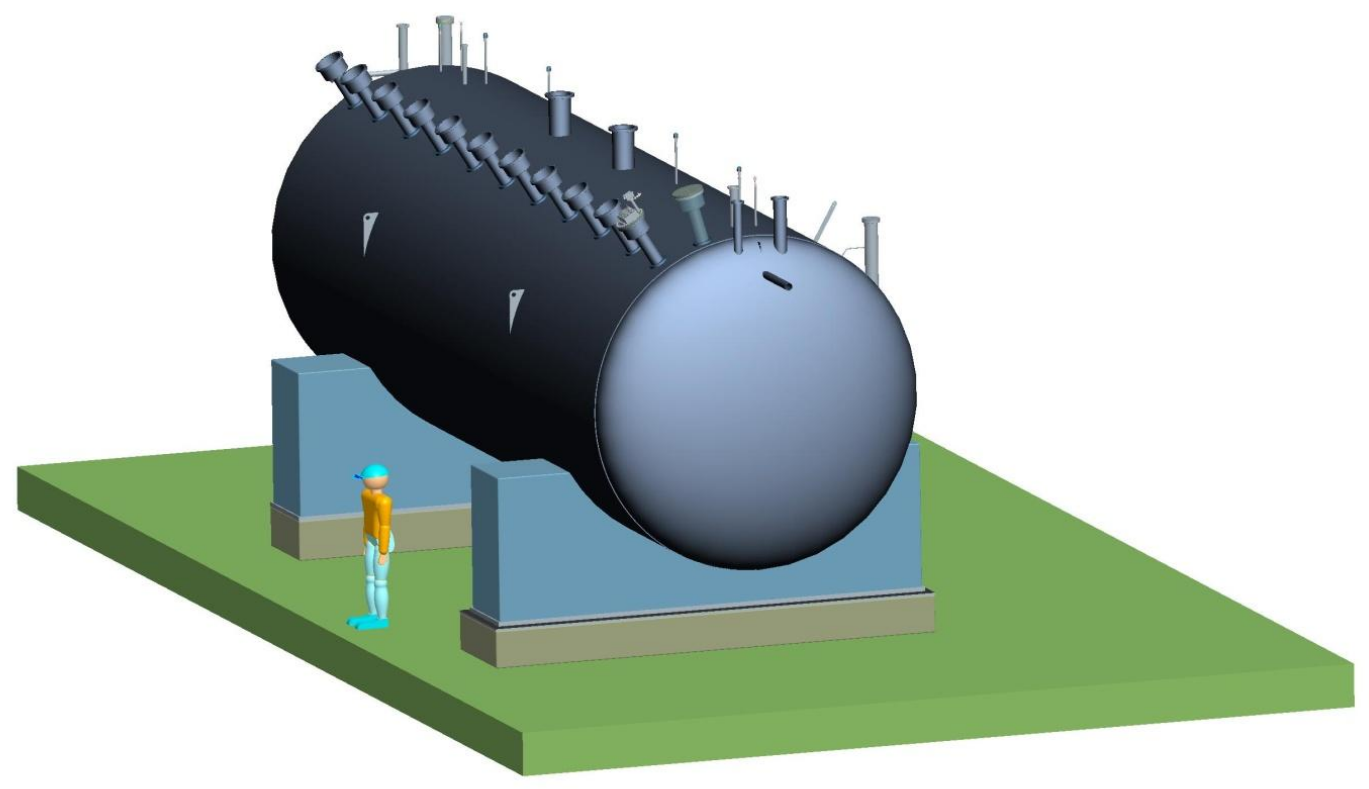

Figure 7.4 Foam saddles bonded to 120 degree arcs of the MicroBooNE cryostat cylinder. The vessel wall foam insulation is not shown.

The two 200" by 36" bottom surfaces of the saddles bond to steel plates. The support plate nearest to the removable end anchors to the floor. The other end attaches to the mounting surface to allow for axial thermal shrinkage, either by rollers or sliding lubricated plates. Both rollers and plates anchor in a manner that does not allow motion transverse to the vessel axis. 


\subsection{Summary of Mechanical Properties of Cryostat}

\subsubsection{Sizes and Weights}

Vessel overall length

Vessel straight section

Outer diameter

Wall thickness, material

Vessel internal volume

Ullage gas volume

Vessel surface area

Vessel weight

Weight of $75 \mathrm{~m}^{3}$ foam Insulation

Weight of LAr

Weight of LAr Vessel fully loaded
481 inches, $12.2 \mathrm{~m}$

425.2 inches, $10.85 \mathrm{~m}$

152 inches, $3.9 \mathrm{~m}$

1 inch nominal, 304 stainless

132975 liters, 35131 gal

$8.9 \%$ of total

$1490 \mathrm{ft}^{2}, 138 \mathrm{~m}^{2}$

67242 pounds

6432 pounds

373120 pounds, 169.6 tonnes

446697 pounds, 203 tonnes

\subsubsection{Deflection and Stress of Fully Loaded Vessel}
Maximum deflection at $30 \mathrm{psig}$
$5.9 \mathrm{~mm}$ max., 0.24 inches
Maximum stress at 30 psig
97 MPa max., 14 kpsi, welded dome
Safety factor at $30 \mathrm{psig}$
2.1

\subsubsection{Deflection and Stress of Saddles Supporting the Fully Loaded Vessel}

Maximum deflection, no LAr

$0.6 \mathrm{~mm}$ compression

Maximum deflection, full load

$2.8 \mathrm{~mm}$ compression

Maximum stress

$0.5 \mathrm{MPa}, 73 \mathrm{psi}$

\subsubsection{Seismic Analysis for 0.1g (UBC Zone 1)}

Seismic forces applied axially: Both saddles share the load (if one saddle takes the total load, multiply by 2.) 
Maximum vessel deformation

Maximum vessel stress

Maximum saddle deformation

Maximum saddle shear stress
$6.4 \mathrm{~mm}, 0.26$ inches, top center

$93 \mathrm{MPa}, 13.5 \mathrm{kpsi}$, welded dome area

$2.0 \mathrm{~mm}, .080$ inches

$0.36 \mathrm{MPa}, 52 \mathrm{psi}$

Seismic force applied laterally over the length of the vessel.

Maximum vessel deformation

Maximum vessel stress

Maximum saddle deformation

Maximum saddle stress
$8.2 \mathrm{~mm}, 0.33$ inches

$114 \mathrm{MPa}, 16.5 \mathrm{kpsi}$, welded dome area

$4.7 \mathrm{~mm}, 0.19$ inches

$0.42 \mathrm{MPa}, 60 \mathrm{psi}$

All deflections and stresses lie well within limits, with toppling not a factor. 


\section{Time Projection Chamber (WBS 1.4)}

\subsection{Introduction}

A liquid argon time projection chamber (LArTPC) exploits features of pure liquid argon that allow ionization electrons to be transported along uniform electric field lines over several meters to wire chamber planes. The low rate of diffusion in LAr preserves the spatial structure of ionization produced along charged particle tracks. A LArTPC delivers excellent neutrino interaction characterization and superior background rejection capabilities, and also serves as a high density homogeneous target. The technology provides precise space point determinations of charged particles for tracking, as well as detailed determination of local ionization energy loss for particle identification and calorimetry.

\subsubsection{Overview}

The MicroBooNE LArTPC design allows ionization electrons from charged particle tracks in the active LAr volume to drift to and be sampled by a three-plane wire chamber. Wire plane readout electronics amplify induction and collections signals on the wires and send sampled charge collection waveforms to the data acquisition (DAQ) system. This chapter provides details on the TPC mechanical and high voltage distribution for the TPC. Chapter 10 describes the readout electronics and DAQ system. The mechanical system consists of the cathode plane, field cage, wire chamber planes, and support elements. The drift high voltage (HV) system consists of the HV power supply, the HV feedthrough (HVFT) that carries $-128 \mathrm{kV}$ from outside the detector to the cathode plane, and a HV low pass filter for noise reduction. Figure 8.1 depicts the TPC elements inside the MicroBooNE cryostat. 


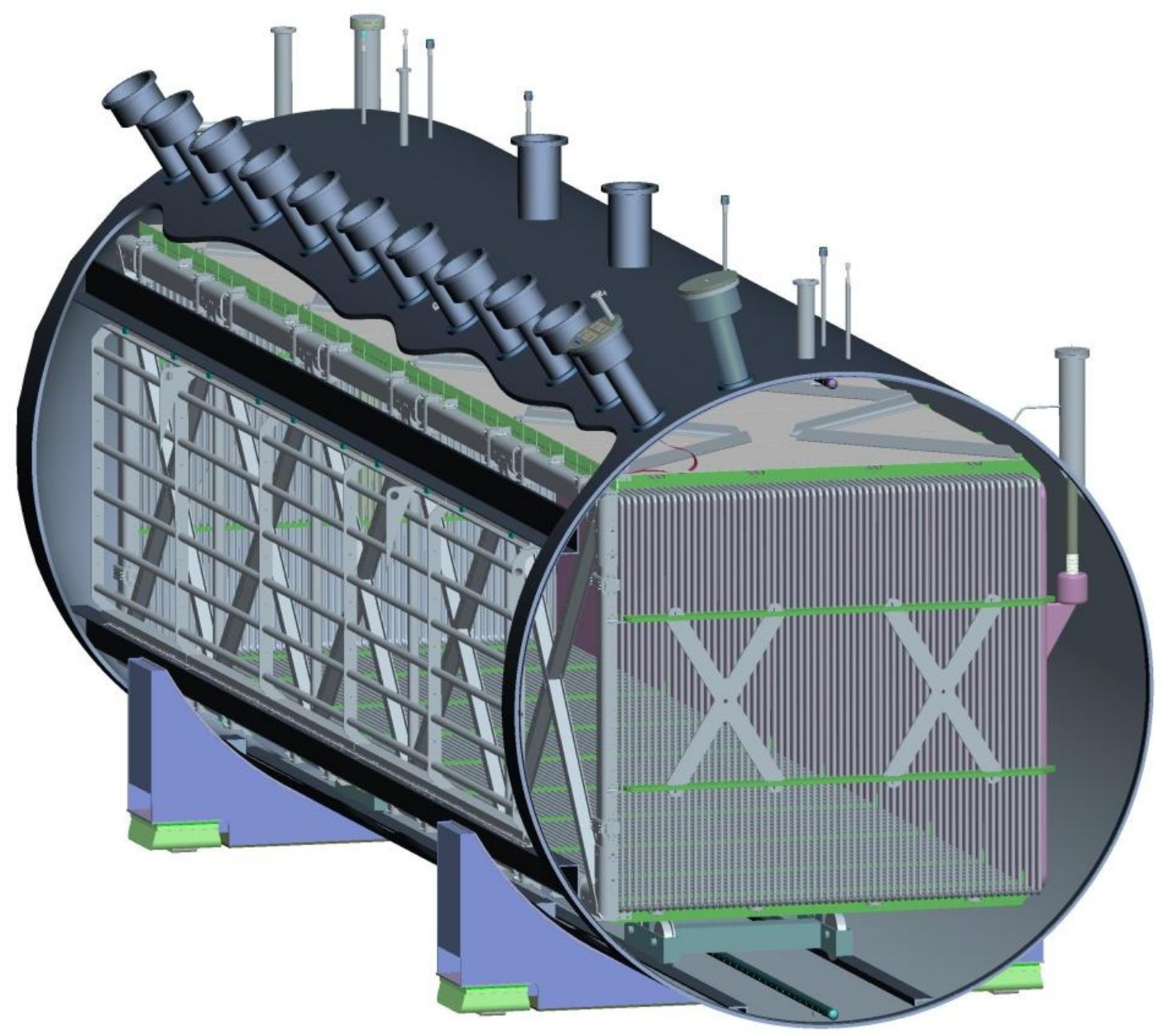

Figure 8.1 The TPC inside the cryostat looking up the beamline from the downstream side. The cathode plane is on the right (beam-left). The wire planes and PMT array are on the left (beam-right). The TPC HV feedthrough is top-right (beam-left), and the TPC signal feedthroughs are shown top-left (beam-right).

A (2.33 m height $) \times(2.56 \mathrm{~m}$ width $) \times(10.37 \mathrm{~m}$ length $)$ rectangular solid defines the $61.8 \mathrm{~m}^{3}$ active volume of the TPC, which encompasses 86 tons of liquid argon when operational. The TPC cathode plane forms the vertical boundary of the active volume on the left side of the detector when viewed along the neutrino beam direction ("beam-left"). Three parallel vertical sense wire planes are mounted on the "beam-right" side of the active volume. The wires in the "Y" plane are oriented vertically, while wires in the " $U$ " and "V" planes are oriented \pm 60 degrees, respectively, with respect to vertical. Ionization electrons drift from beam-left to beam-right, reaching in turn the $\mathrm{U}, \mathrm{V}$, and $\mathrm{Y}$ planes. The drifting electrons induce charge on the $\mathrm{U}$ and $\mathrm{V}$ induction plane sense wires as they drift by and deposit their negative charge on the $\mathrm{Y}$ collection plane sense wires.

The TPC HVFT occupies a position near the beam-left downstream top corner of the TPC. The TPC signal feedthroughs line up along the top beam-right boundary of the TPC. 


\subsubsection{Technical Design Criteria}

The following summarizes the main design criteria for the TPC:

- The target volume enclosed by the TPC must be large enough to achieve the physics goals of the experiment. The aspect ratio of the fiducial volume is optimized to meet the scientific goals of the experiment.

- The wire pitch $(3 \mathrm{~mm})$ of the three wire chamber planes should satisfy the development goals for liquid argon TPC development.

- The wire chambers must be constructed to ensure that no wires break inside the cryostat volume.

- The TPC electric field must produce an electron drift speed of $1.6 \mathrm{~mm} / \mu \mathrm{s}$ in liquid argon.

- The TPC field cage must produce a uniform field inside the TPC active volume excluding the region within $5 \mathrm{~cm}$ of the field cage tubing.

- The HV system must provide $-128 \mathrm{kV}$ on the cathode.

- The HV system must operate with a ripple voltage sufficiently low so that a $5 \mathrm{~m}$ long Uplane wire collects fewer than 100 electrons injected from the HV system in $1 \mu \mathrm{s}$.

- The electric field must not exceed $100 \mathrm{kV} / \mathrm{cm}$ anywhere in the LAr to prevent electrostatic breakdown.

Additionally, the design, construction, and operation of the MicroBooNE TPC should inform the HEP community as much as possible about building more massive LArTPCs in the future.

\subsection{TPC Technical Design}

\subsubsection{Time Projection Chamber}

Figure 8.1 shows a three dimensional rendering of the design of the TPC. The detector cryostat houses the TPC, which surrounds a LAr active volume in the shape of a rectangular box of dimensions $(2.33 \mathrm{~m}$ vertical $) \times(2.56 \mathrm{~m}$ horizontal $) \times(10.37 \mathrm{~m}$ longitudinal $)$ slightly offset from the center towards the beam-right side of the cryostat to give a larger clearance between the HVcathode plane and the vessel walls. Figure 8.2 shows the key components of the TPC, and the following sections describe these components in more detail.

\subsubsection{Cathode Plane}

The cathode plane (Figure 8.3) on beam left inside the cryostat consists of a framework of stainless steel (type 304) holding an array of nine rectangular stainless steel sheets mounted on one side. Round tubes of $50.8 \mathrm{~mm}$ OD $3.2 \mathrm{~mm}$ wall thickness make up the outer edges of the 
cathode frame, while $50.8 \mathrm{~mm} /$ side square tubes of the same wall thickness serve as inner vertical members. The $1.6 \mathrm{~mm}$ thick cathode sheets (facings) bolt to the framework. Shims allow adjustment of each sheet to form a flat conductive surface $10.47 \times 2.43 \mathrm{~m}^{2}$ in area. The $-128 \mathrm{kV}$ bias voltage on the cathode plane leads to a uniform electric field of $500 \mathrm{~V} / \mathrm{cm}$ in the horizontal direction throughout the active LAr volume. A metal "cup" attached to the frame of the cathode at the cryostat opening end provides the electrical connection the HV power supply through the HV feedthrough.

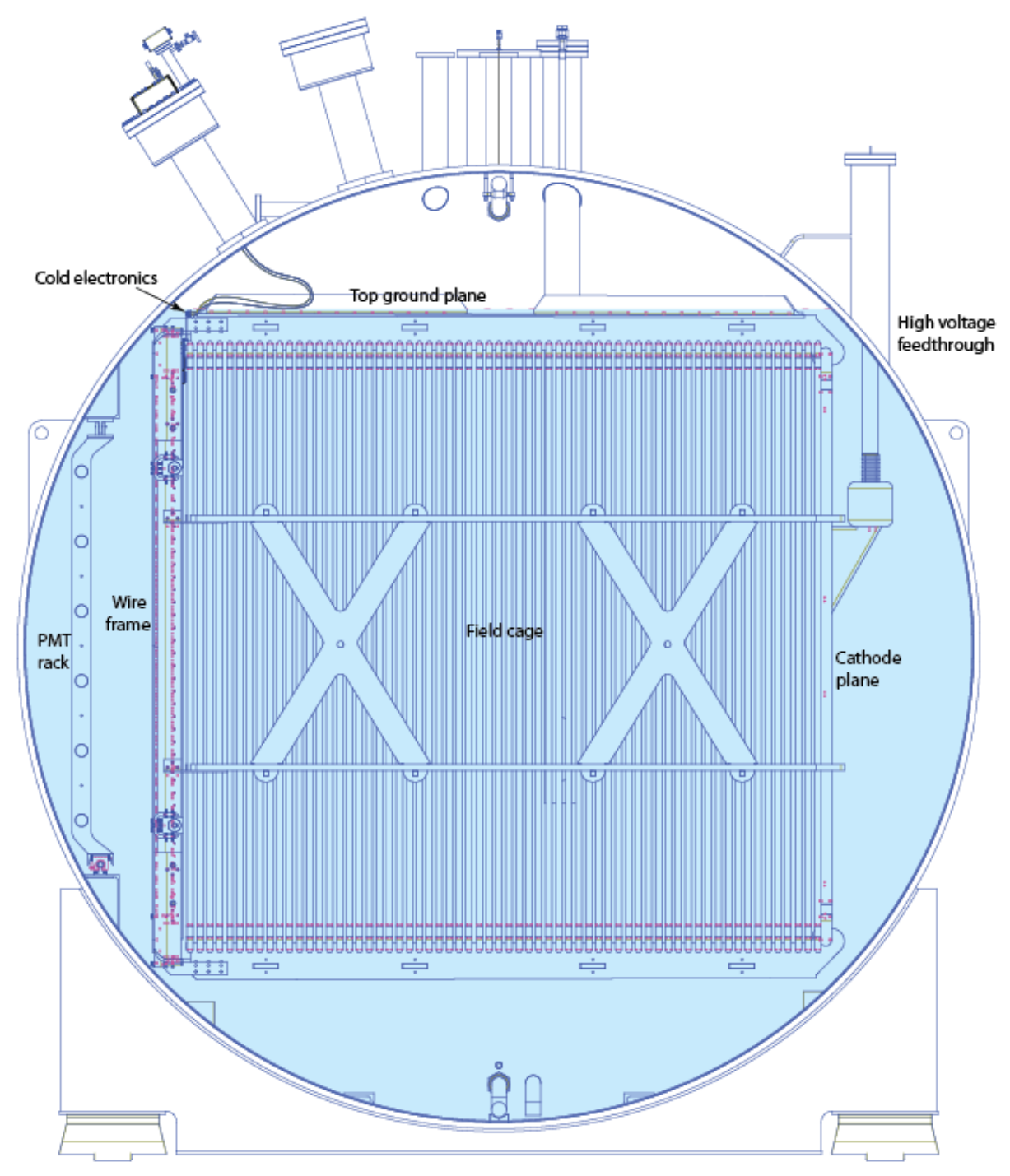

Figure $8.2 \mathrm{~A}$ cross sectional view of the TPC installed inside the cryostat. The beam direction is into the paper.

Insulating legs under the second and the seventh inner vertical frame members support the cathode plane on two platforms. The downstream end of the platform allows the cathode to slide beam-left and beam-right, while the upstream end allows the cathode to slide in both directions on the horizontal plane. This play accommodates the difference in thermal contraction between the TPC and the cryostat. 


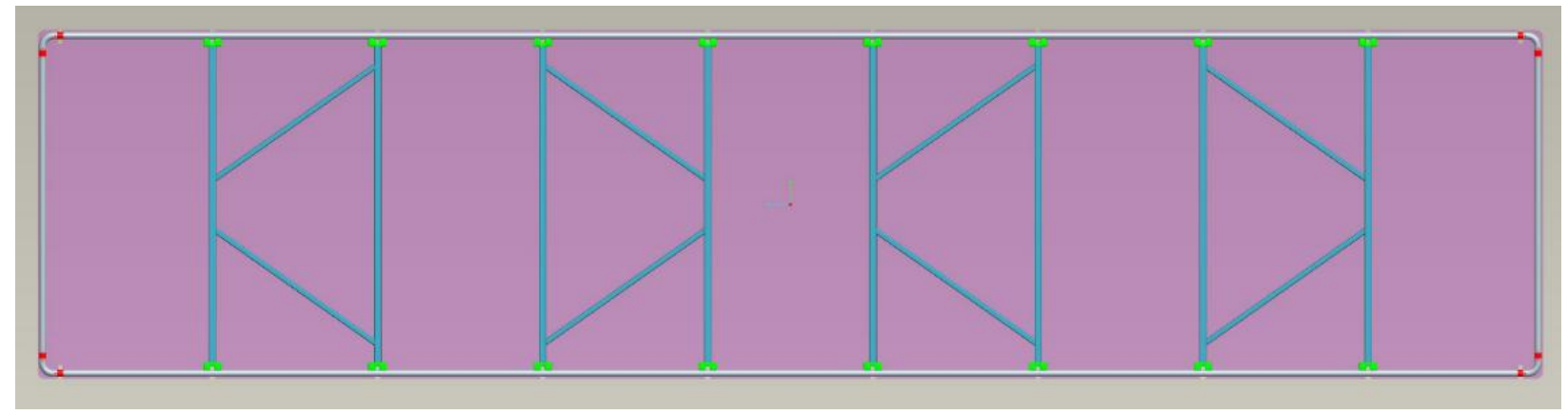

Figure 8.3 The TPC cathode plane.

\subsubsection{Field Cage}

The HV drift cage must provide a uniform $500 \mathrm{~V} / \mathrm{cm}$ electric field over the TPC active volume to maintain linearity between drift distance and time of the ionization. The MicroBooNE TPC geometry dictates a $-128 \mathrm{kV}$ voltage on the cathode side to produce this field over the $2.56 \mathrm{~m}$ maximum possible drift distance, defined as the distance from the cathode surface to the $\mathrm{Y}$ wire plane. To maintain mechanical strength and to simplify construction, the field cage employs arrays of $2.5 \mathrm{~cm}$ OD stainless steel tubes with a $4.0 \mathrm{~cm}$ center-to-center separation for its electrodes. The voltage increases towards $0 \mathrm{~V}$ moving away from the cathode plane in $2 \mathrm{kV}$ steps from tube-to-tube to provide adequate field uniformity.

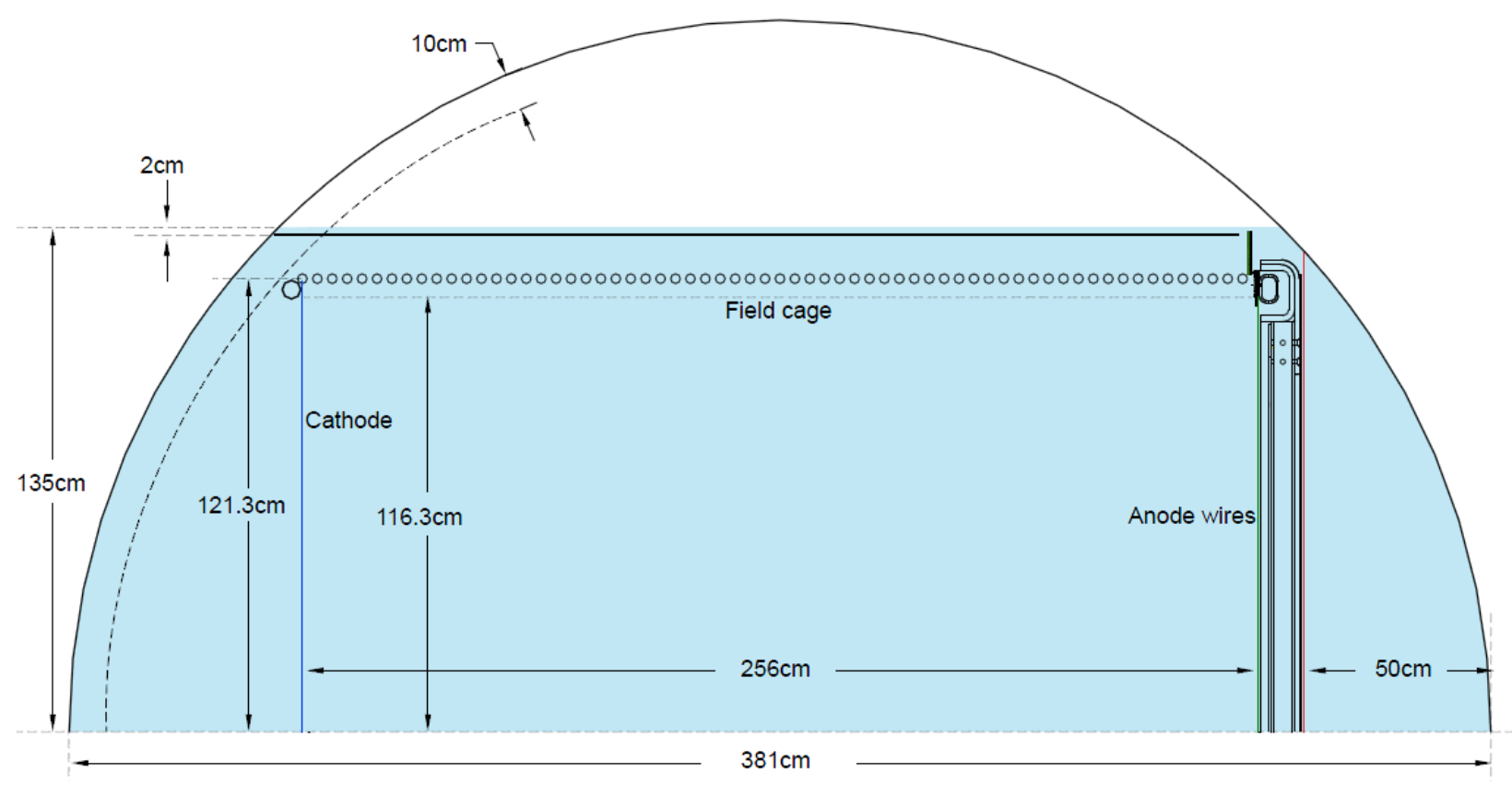

Figure 8.4 A schematic cross section view of the top half of the cryostat, viewed along the beam direction.

The high bias voltage on the cathode end of the field cage creates a very high electric field in both the liquid argon and in the argon gas in the cryostat ullage. The relatively low breakdown voltage of the argon gas creates the possibility of having high voltage discharge through the 
argon gas to the cryostat wall. To mitigate this risk, a ground plane placed $2.0 \mathrm{~cm}$ below the liquid level shields the field cage from the argon gas. This ground plane incorporates $1.6 \mathrm{~mm}$ thick perforated stainless sheets in its construction to allow gas bubbles to pass through.

The top and bottom planes of the drift cage span the $10 \mathrm{~m}$ length of the TPC. To keep the tubes from sagging due to their own weight, and to maintain a uniform spacing between the tubes, G10 beams with uniformly spaced holes holding the drift cage electrodes at periodic intervals serve as stiffening elements. In addition to carrying the weight of the field electrodes, the G10 support structures also tie the HV cathode plane to the wire frame, maintaining the distance between the wire frame and the cathode plane. The centers of the field cage tubes extend $5 \mathrm{~cm}$ beyond the edge of the TPC to improve the uniformity of the drift field at the edges of the active volume.

The G10 beams establish the mechanical structure of the TPC along the drift field direction, while the stainless steel field cage electrodes determine the structure in the beam and vertical directions. The materials imply that anisotropic thermal contraction occurs when the structure is cooled down. This poses a challenge to designing the diagonal bracing structures on the field cage that keep the TPC square. Instead of directly tying the cathode and wire frame together diagonally, the design employs smaller " $X$ " shaped G10 bars mounted between two of the G10 beams holding the tubes (Figure 8.5) to take up the asymmetric contraction of the structure when it cools down. A slight deflection of the G10 beams along the direction of the field cage tubes results during this process. 


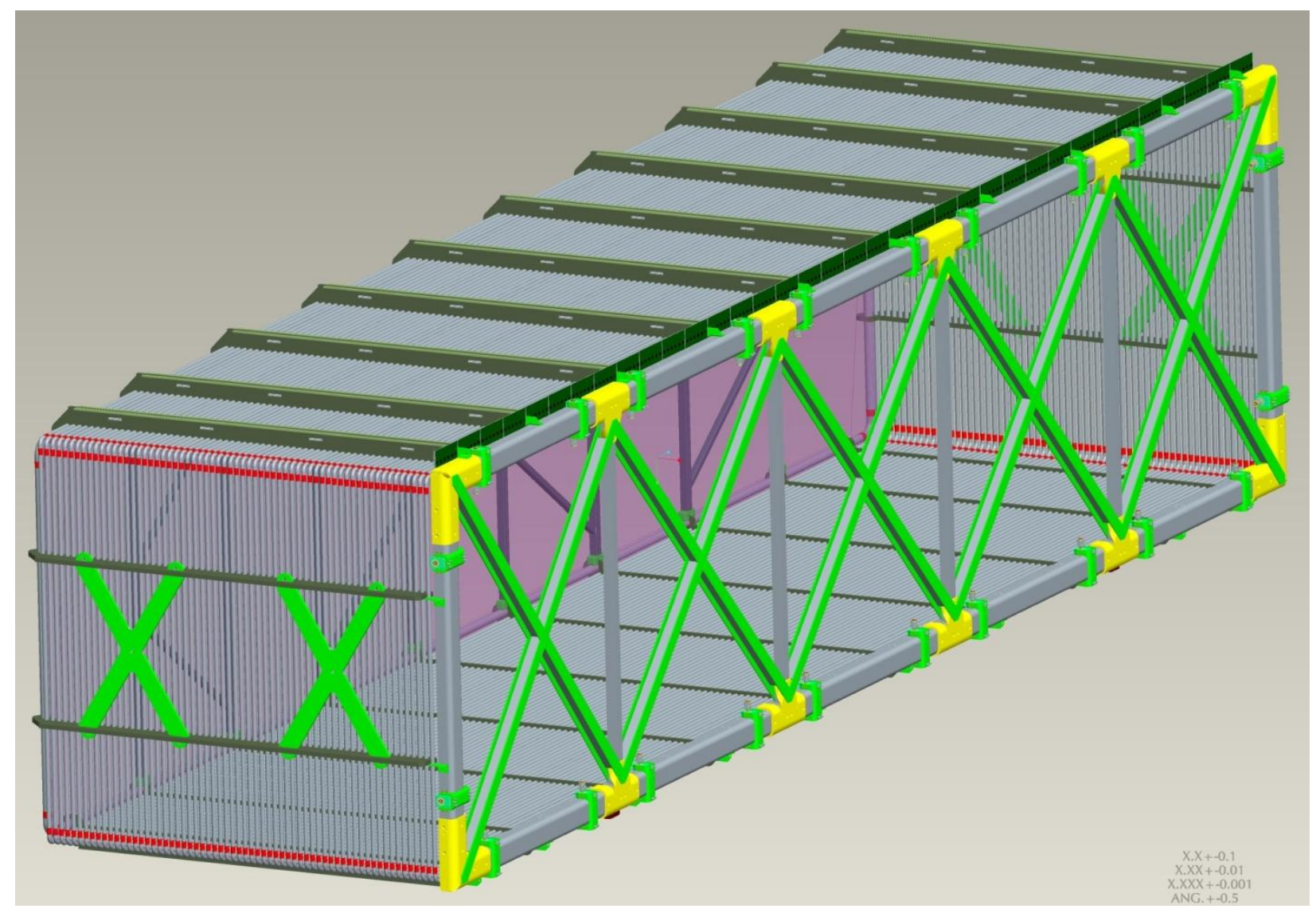

Figure 8.5 A rendering of the field cage structure.

To reduce the cost and weight of the field cage structure the field cage uses $0.5 \mathrm{~mm}$ wall thickness TIG welded stainless steel tubes with venting holes drilled periodically along the length of each tube to prevent trapping of gas. Stainless steel elbows with custom made couplings connect these tubes at the four corners to form a closed loop.

A resistor divider chain supplies the potential for the field cage electrodes. To reduce the field distortion caused by a possible resistor failure, four equal value $1 \mathrm{G} \Omega$ resistors, with each resistor rated at $5 \mathrm{kV}$ and $1 \mathrm{~W}$, make parallel connections between neighboring electrodes. The resulting four chains of resistors solder onto a set of printed circuit boards that then attach to the field cage tubing both electrically and mechanically with screws. The entire resistor chain dissipates about $1 \mathrm{~W}$ of heat inside the cryostat. To avoid possible argon gas bubbles entering the drift volume, the resistor divider boards mount onto an upper edge of the end cap field cage wall.

The drift cage electric field has been simulated with two-dimensional electrostatic field simulator Opera-2D v13. The Opera calculation verifies good field uniformity, with a slight deterioration at the corners. The maximum field on the cathode outer edge reaches $30 \mathrm{kV} / \mathrm{cm}$. To quantitatively estimate the uniformity of the field along the lines of drift, the Opera code computes the absolute value of the electric field along straight lines in the x-direction at $0,5,10$, and $15 \mathrm{~cm}$ from the top edge of the active volume. Figure 8.6 summarizes the results. A maximum 
field variation of $\pm 1 \%$ exists at the top edge, where the effects of the field cage tubes are clear. The field variation falls below $\pm 0.2 \%$ at $10 \mathrm{~cm}$ from the edge and further inward.

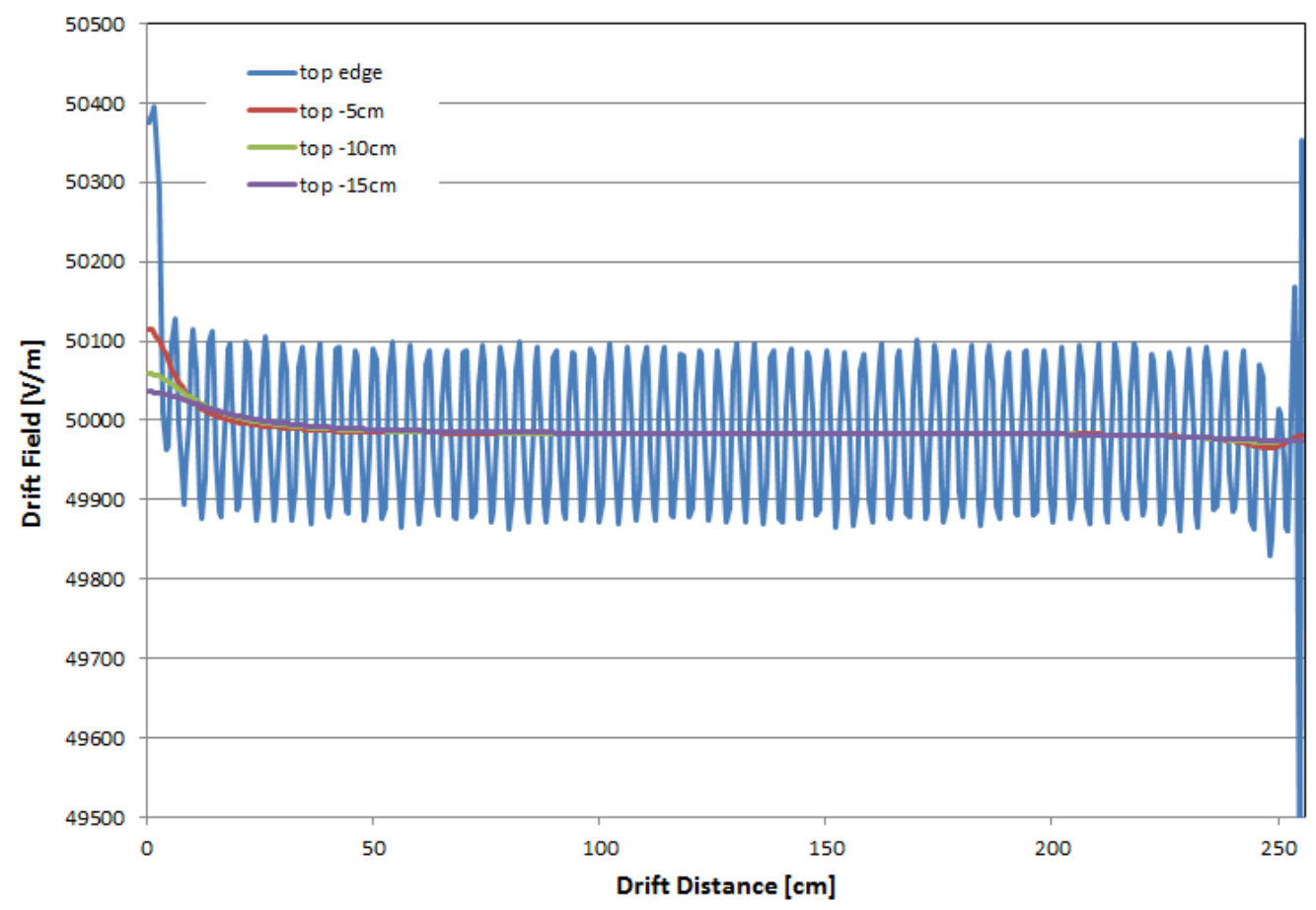

Figure 8.6 Drift field uniformity along lines in the drift direction at various distances from the top edge of the active volume. 


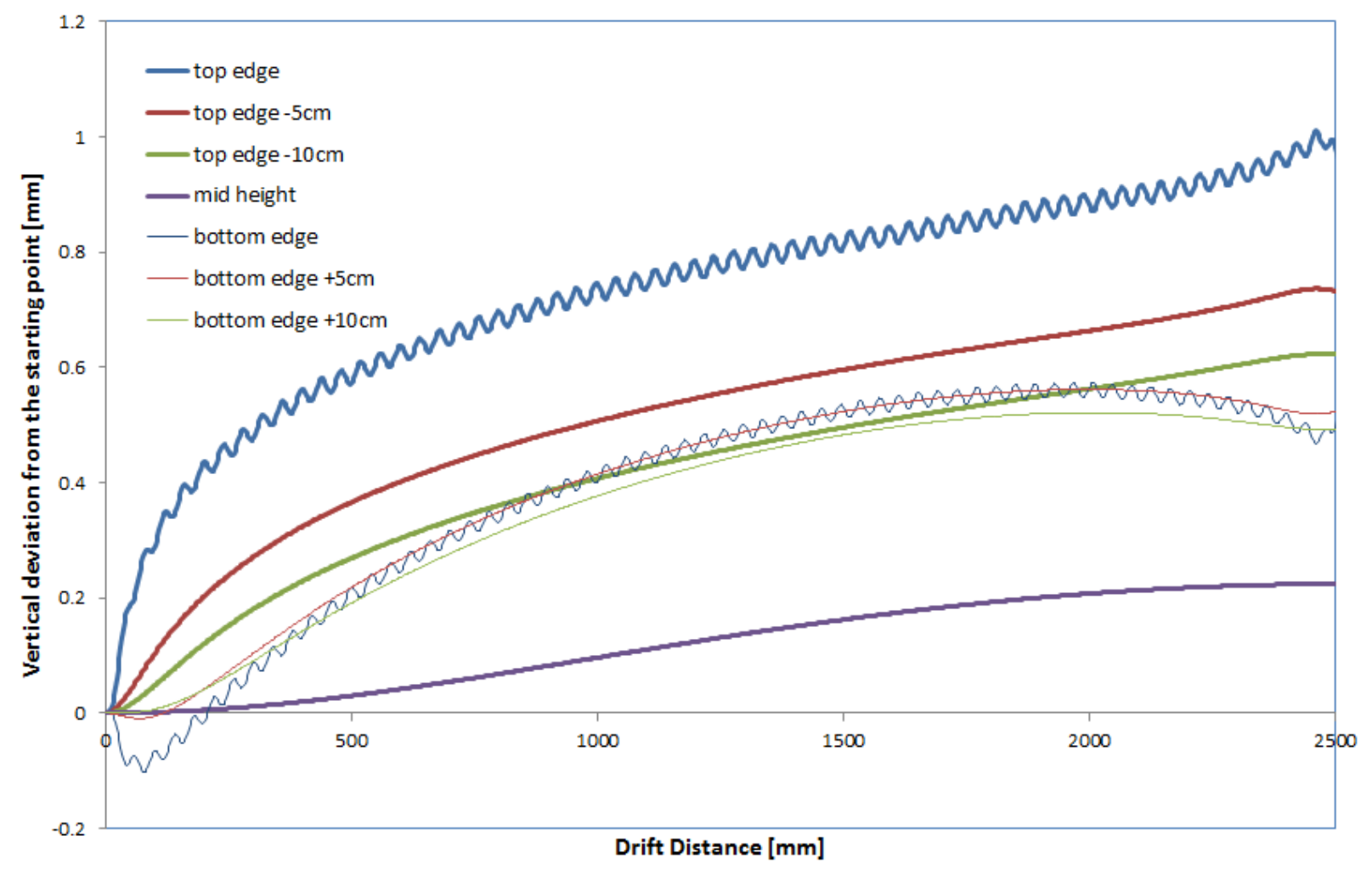

Figure 8.7 Error in electron drift as a function of the drift distance for several starting point at the cathode surface.

The presence of the top ground plane causes a slight asymmetry in the drift field. As illustrated in Figure 8.7, drifting electrons acquire a vertical displacement of order $1 \mathrm{~mm}$. A $\pm 0.5 \%$ random variation in the resistance values between divider taps produces a comparable distortion. MicroBooNE DocDB 1346 provides more details.

\subsubsection{The Wire Planes}

Three readout planes, spaced by $3 \mathrm{~mm}$, form the beam-right side of the detector, with the 3456 $\mathrm{Y}$ wires arrayed vertically and the $2400 \mathrm{U}$ and $2400 \mathrm{~V}$ wires oriented at \pm 60 degrees with respect to the $\mathrm{Y}$ wires. All planes have a wire separation of $3 \mathrm{~mm}$ within the plane. Bias voltages placed on the three wire planes allow all electrons pass through the $\mathrm{U}$ and $\mathrm{V}$ planes to be collected by the Y plane. A grounded mesh with $>80 \%$ optical transparency placed $1 \mathrm{~cm}$ behind the Y-plane shields against field irregularities and blocks electrons from drifting to the Y plane wires from outside the active volume.

All readout planes use $150 \mu \mathrm{m}$ stainless steel (type 304V) wire with $2 \mu \mathrm{m}$ thick copper plating covered with a thin flash of gold to prevent copper oxidization. The copper plating decreases the resistivity of the wire from $40 \Omega / \mathrm{m}$ to $3 \Omega / \mathrm{m}$ in liquid nitrogen, reducing series noise in the readout electronics. Every wire terminates at each end via several twists around a small brass ring (Figure 8.8). This termination results in a wire break strength above $3.5 \mathrm{~kg}(34.3 \mathrm{~N})$ at room 
temperature, reaching above $4.5 \mathrm{~kg}(44.1 \mathrm{~N})$ in liquid nitrogen. The $0.7 \mathrm{~kg}(6.86 \mathrm{~N})$ of tension established in each wire is small enough to prevent wire breakage during cool down, but large enough to limit the maximum wire sag due to gravity to under $0.5 \mathrm{~mm}$ for any $5 \mathrm{~m}$ long $\mathrm{U}$ or $\mathrm{V}$ wire. Wire length must satisfy a tolerance equal to the greater of $\pm 0.02 \%$ or $\pm 0.25 \mathrm{~mm}$; this can be achieved with the automated wire winding machine shown in Figure 8.9.

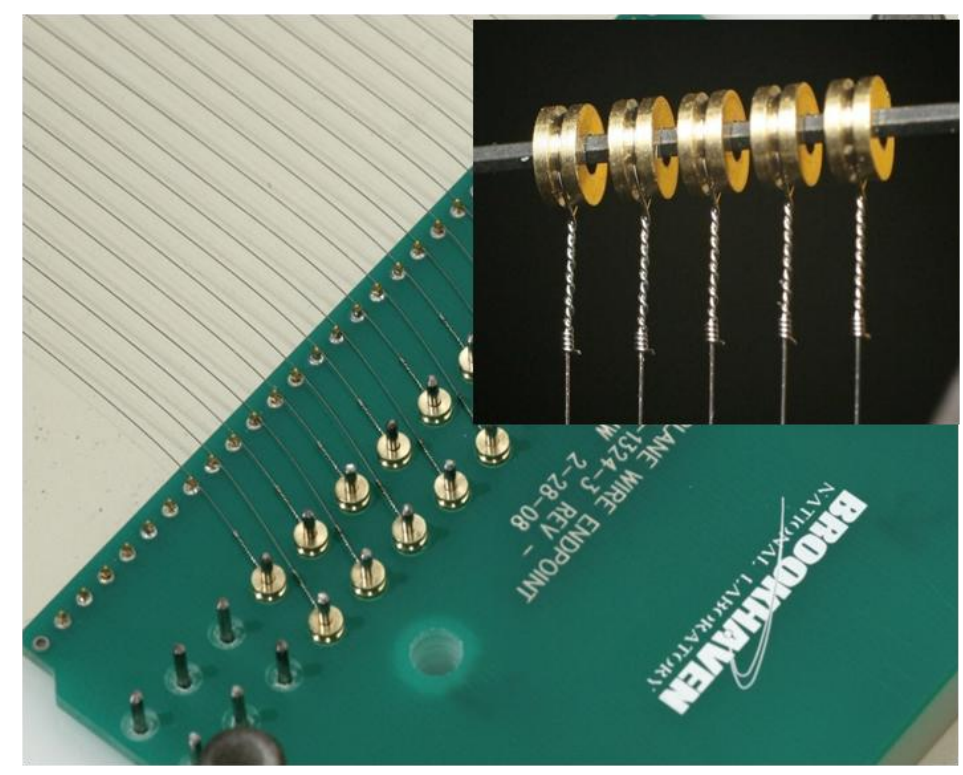

Figure 8.8 Wire termination and a wire carrier board.

Two automated winding machines, one for fixed length wires, the other for variable length wires, can terminate wires at a rate of about 30 seconds per wire. The winding machine sets the length of each wire at the nominal $1.0 \mathrm{~kg}(9.8 \mathrm{~N})$ tension. Each terminated wire receives a stress test at $2.5 \mathrm{~kg}(24.5 \mathrm{~N})$ for a minimum of 10 minutes to ensure its mechanical integrity. Destructive breaking strength tests performed at regular intervals check for systemic issues. 


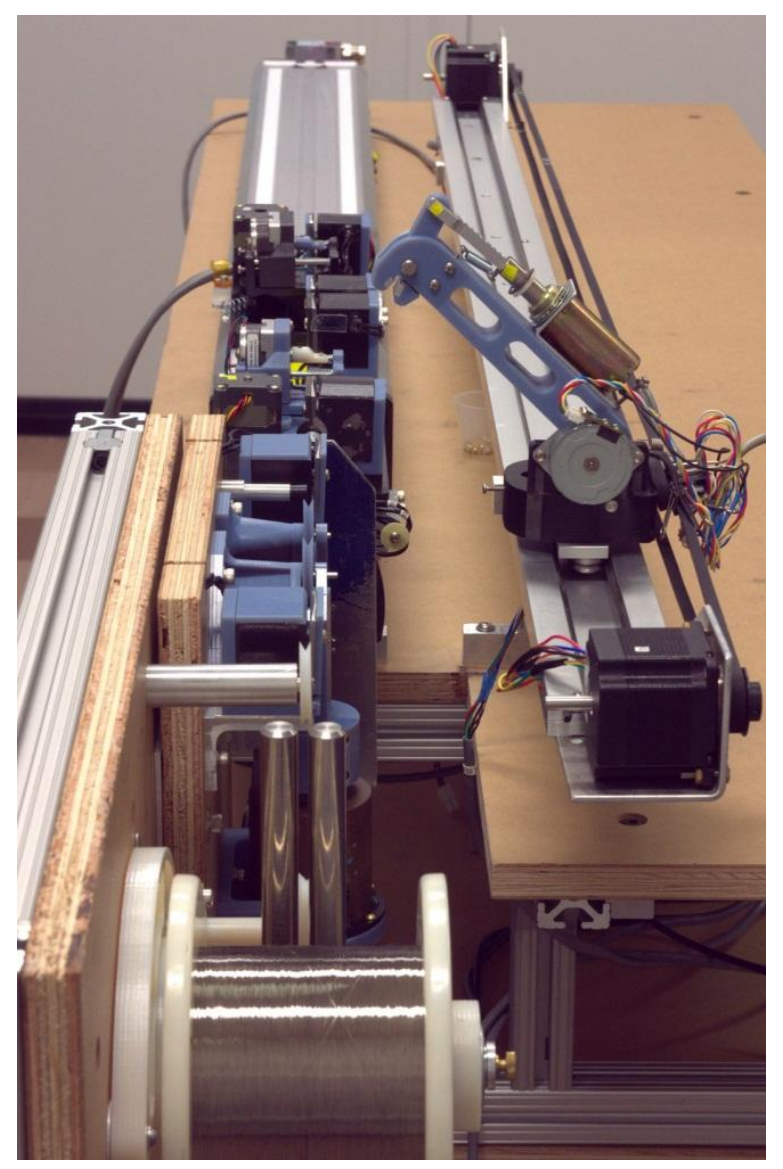

Figure 8.9 A photo of the prototype winding machine capable of terminating wires up to about $1 \mathrm{~m}$ in length.

Specially designed wire holder modules hold the installed wires to streamline production, storage, and final assembly. Each wire holder module holds 32 wires in Y, and 16 wires in U, and 16 wires in V. A wire holder module consists of a stack of two printed circuit boards with a total thickness of $3 \mathrm{~mm}$, as shown in Figure 8.8. The wire terminations are encapsulated in rows of circular cavities in the wire holder. A row of gold-plated press-in pins defines the location of the wires as they exit the wire holder. Electrical signals from the press-in pins route to a connector at the top edge of the board.

A proper ratio of electric field on both sides of each wire plane must be maintained to prevent collection of electrons on the $\mathrm{U}$ and $\mathrm{V}$ planes of wires. Simulations show that bias voltages of $200 \mathrm{~V}, 0 \mathrm{~V}$, and $+440 \mathrm{~V}$ for the $\mathrm{U}, \mathrm{V}$, and Y planes, respectively achieve $100 \%$ transmission of electrons through the first two induction planes. Blocking capacitors and high value resistors for each wire on the $\mathrm{U}$ and $\mathrm{Y}$ planes protect the readout electronics from the bias HV.

Despite the fact that each wire is stress-tested, the possibility exists that a wire could break after complete detector installation in the closed cryostat. In the worst case, a broken wire could contact with the cathode frame, held at $-128 \mathrm{kV}$, and create a short circuit that would disable the entire TPC. To mitigate this risk, an intense quality control procedure has been put in place. 
MicroBooNE DocDB-1780 describes in details the procedures and tests that will take place during fabrication, winding and assembly in order to prevent any wire breakage.

Each assembly to be installed consists of a group of wires with two wire carrier modules at the ends secured to a mounting plate; the entire assembly loads with others into a wire storage module. Three different wire storage modules hold all the wire carriers needed for the entire detector for storage and subsequent cleaning, packing, and transportation to the assembly site at Fermilab.

\subsubsection{Wire Frame}

Figure 8.10 illustrates the design of the wire frame structure. The entire frame divides into five sections. Each outer frame section is fabricated from stainless steel "C"-bars $16 \mathrm{~mm}$ thick. The "T" and "L" stainless steel joints (shown in yellow in Figure 8.10a) are also fabricated by a CNC break-press. Inside each of the outer C-channel frame section, a stainless steel tensioning bar (shown in brown in Figure 8.10b) mounts through two guiding rods (Figure 8.10c) with a set of adjustment screws that enable the tensioning bar to be moved in all directions for alignment and wire tensioning purposes. To ensure the wire planes are mounted precisely on the wire frame, the tensioning bar adjustment through the guiding rods takes place under the direction of a team of surveyors at the Fermilab assembly site. The wire carrier modules mount onto the tensioning bars one wire plane at a time, with the tensioning bars retracted. After all the wire carriers have been attached, a stainless steel compression bar screws onto the tensioning bars, locking the wire carriers in between. Tension adjusting screws distributed along the length of the wire frame extend the tensioning bars, with wire tension monitored by a tension measuring device, until the achievement of nominal wire tension. This design allows major frame structures to be fabricated and assembled with relatively low dimensional tolerances, while ensuring good precision on the placement of the wire planes through post-assembly adjustments.

Two small mounting platforms on the cryostat at the one-fifth and four-fifths positions along the length of the TPC support the wire frame in a manner analogous to that used for the cathode frame. The downstream support point downstream near the opening end of the cryostat remains fixed, while the upstream support allows the frame to slide along the length of the cryostat. The two platform supports for the wire frame combined with the two platforms supports under the cathode frame enable entire weight of the assembled TPC to be supported at four points by the cryostat, with no other mechanical support necessary. This support scheme minimizes the impact of the deformation of the filled cryostat on the TPC, allows differential expansion and contraction between the TPC and the cryostat without distortion, and enables the assembled TPC to be inserted into the cryostat with relative ease. 

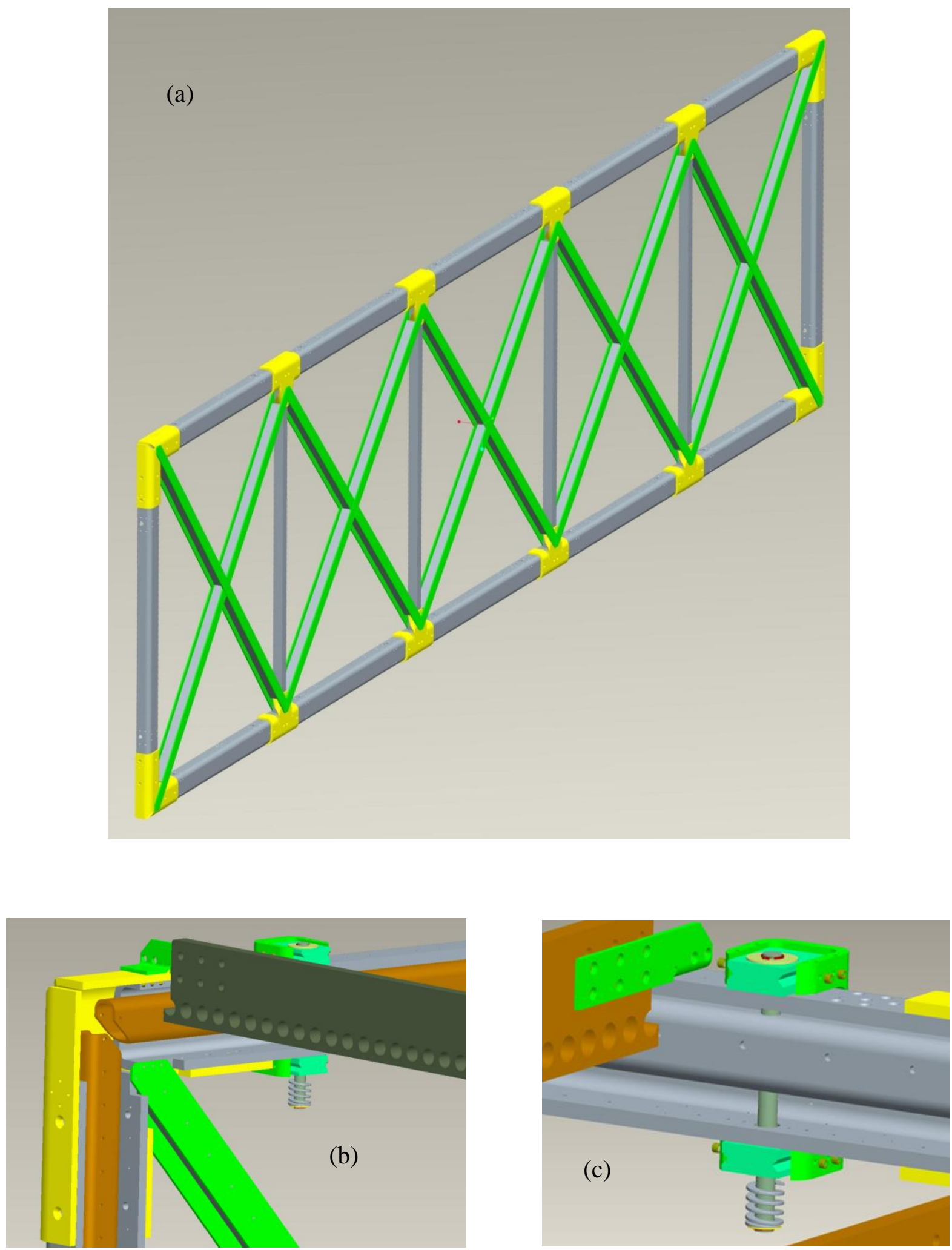

Figure 8.10 (a): A rendering of the TPC showing the wire frame, with $T$ and $L$ joints shown in yellow. (b): detail view of a wire frame corner showing tensioning bars. (c): detail of the tensioning bar guiding rod with adjustment mechanism 


\subsubsection{High Voltage System}

The MicroBooNE TPC requires an electrostatic potential of $-128 \mathrm{kV}$ at the cathode plane. The High Voltage (HV) system consists of a HV power supply, a noise filter, and a feedthrough (HVFT) through the tank wall into the LAr.

\subsubsection{Power Supply}

Three different power supplies have been considered for the HV system. The table below describes their features.

\begin{tabular}{|c|c|c|c|}
\hline $\begin{array}{l}\text { Manufacturer } \\
\text { Model }\end{array}$ & $\begin{array}{l}\text { Glassman } \\
\text { PK250K014GJ0 }\end{array}$ & $\begin{array}{l}\text { Glassman } \\
\text { LX150R12 }\end{array}$ & $\begin{array}{l}\text { Heinzinger } \\
\text { PNC 250000-1 neg }\end{array}$ \\
\hline Purpose & Testing & Running & Test and Run \\
\hline Cost & At Hand & $12 \mathrm{~K} \$$ & $42.5 \mathrm{k} \mathrm{Eu}=60 \mathrm{k} \$$ \\
\hline Maximum Voltage & 250 kV & $150 \mathrm{kV}$ & $250 \mathrm{kV}$ \\
\hline Maximum Current & $14 \mathrm{~mA}$ & $6 \mathrm{~mA}$ & $1 \mathrm{~mA}$ \\
\hline Voltage Regulation & $12.5 \mathrm{~V}$ & $7.5 \mathrm{~V}$ & 25 Volt \\
\hline Ripple, rms & 65 Volt & 46 Volt & 8 Volt rms \\
\hline Switching frequency & & $70 \mathrm{kHz}$ & $37 \mathrm{kHz}$ \\
\hline Supply voltage & 187-228 V 3-phase & $115 \mathrm{~V}$ & 115 \\
\hline Supply current & $20 \mathrm{~A}$ & $20 \mathrm{~A}$ & \\
\hline
\end{tabular}

The Glassman $250 \mathrm{kV}$ supply (available on loan from the Fermilab Accelerator Division) can be used for testing, with the Glassman $150 \mathrm{kV}$ model the best choice for operational running. The Heinzinger supply remains as an option in case of problems with the Glassman models. The procurement process has started for HV cables and "surge resistor assemblies" for a two-stage ripple noise filter.

\subsubsection{Feedthrough}

The $2.4 \mathrm{~m}$ long HVFT assembly mounts off an 8" OD CF flange, as shown in Figure 8.11. A continuous 2" OD, 1" ID insulating tube made of extruded ultra high molecular weight polyethylene (UHMW-PE) provides the principal electrical seal, with nominal breakdown strength of $400 \mathrm{kV}$. The gas seals around the center conductor and the UHMW-PE tube follow a design used by ICARUS. The center conductor, with its center steel section having slightly larger diameter than the bore of the UHMW-PE tube, presses into the tube. This steel section near the upper end of the HVFT remains at room temperature. The center conductor below this seal section consists of a thin wall tube extending down to the cup-shaped connection receptacle on the cathode plane. The cable from the HV power supply and noise filters enter from the top into a cavity in the center steel section. 


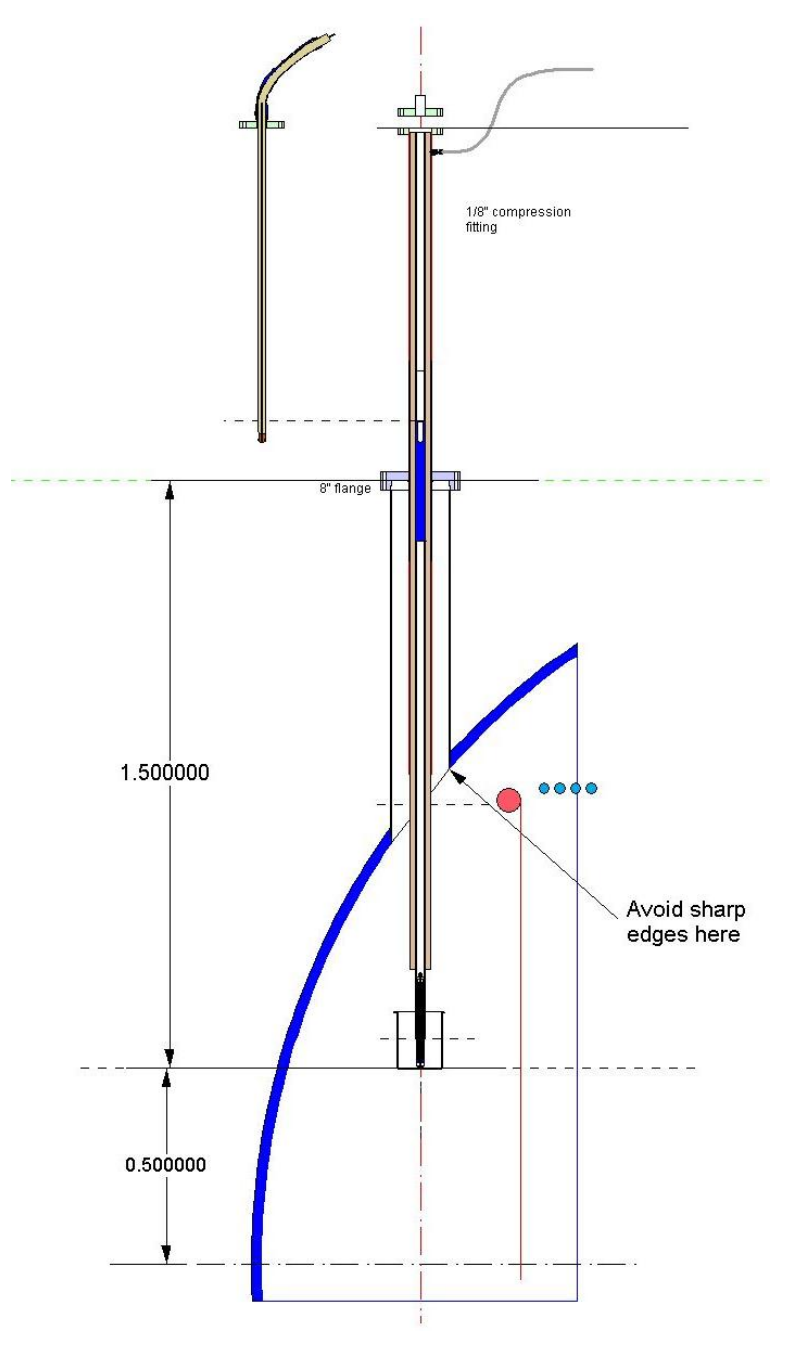

Figure 8.11 Preliminary design of HVFT shown entering the cryostat.

The HVFT installation occurs after TPC installation. The cup-shaped receptacle on the cathode plane accepts the spring loaded bottom end of the HVFT center conductor, as shown in Figure 8.12. Misalignments of up to $1^{\prime \prime}$ can be tolerated in all directions using this scheme, which follows from a proven ICARUS design. 


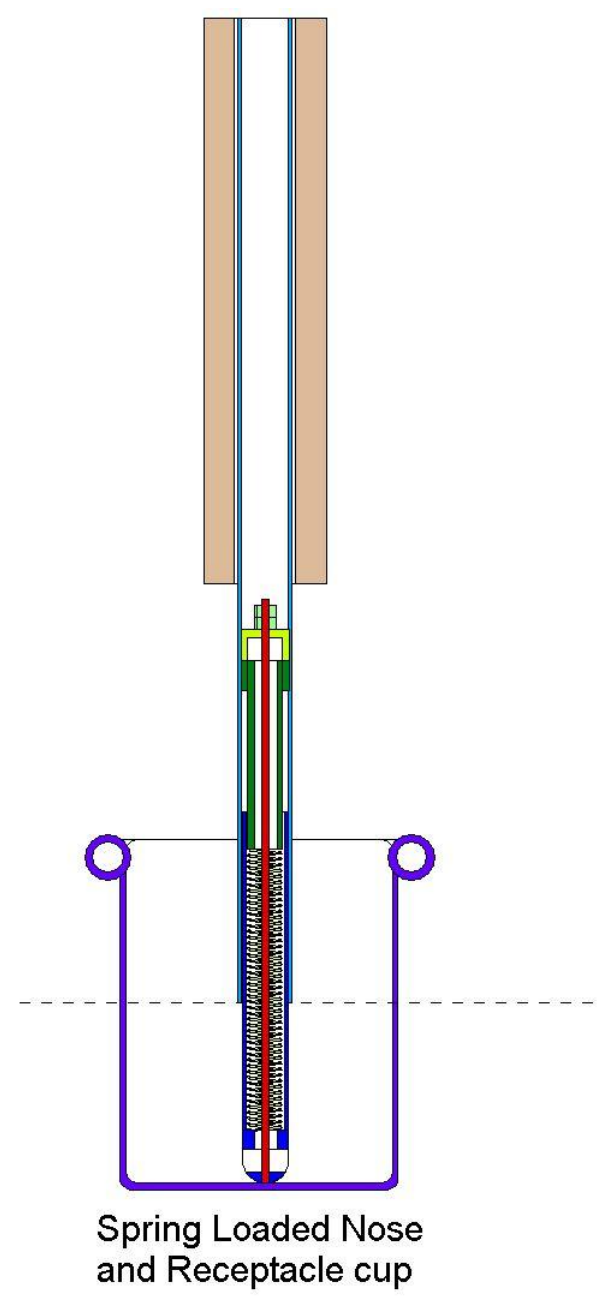

Figure 8.12 Spring contact and receptacle for the HV FT. This design builds off the ICARUS HVFT design.

A second HVFT will be built to the same dimensions as the first one to allow for replacement in the case of failure of the first unit. This replacement can be performed with the cryostat filled with LAr under very low positive pressure. Workers in supplied-air suits unbolt and pull out the old HVFT, and then insert and bolt on the replacement, a quick operation requiring no alignment.

Liquid argon's extremely high $2 \mathrm{MV} / \mathrm{cm}$ dielectric strength makes it very robust against breakdown. Nonetheless, care must be taken to avoid field concentration near sharp edges. As an example, a cylinder of radius $0.1 \mathrm{~mm}$ at $125 \mathrm{kV}$ at a distance of $10 \mathrm{~cm}$ from ground has a surface field of $1.8 \mathrm{MV} / \mathrm{cm}$, nearly enough to initiate breakdown. Therefore, all edges at high potential, inside the tank and out, must be rounded. Parts with rough edges must be placed inside wellrounded corona shields. High electric fields attract small debris. Sharp filings are particularly effective in causing breakdown. Cleanliness of the tank and all its content must be maintained to prevent the accumulation of sharp filings and other small debris that can be attracted by the high 
fields. A plan to test the HV with the complete TPC and HVFT in argon gas prior to closing the cryostat continues to be developed.

Gas breakdown can occur inside the tank if Ar gas bubbles pass through high electric field areas. Argon can boil where the UHMW-PE sleeve and the center conductor of the HVFT enter the liquid. The HVFT design provides for a long exposed UHMW-PE section with a long exposed center conductor at the bottom. Any gas generated at the warm top end of the HVFT should be condensed before contact with $\mathrm{HV}$ in the cold liquid can occur.

Outside the tank, breakdown can occur from the cable connection point in the central steel along the insulated cable to the grounded flange above it. The $60 \mathrm{~cm}$ path along the insulated cable lessens this risk, and further mitigation can be obtained by continuously purging the space with nitrogen gas. The gas purge also removes any water in the gas on the surfaces and in the gas near the warm part of the HVFT to an extremely low level, thus inhibiting flash over and microdischarge.

\subsubsection{Power Supply Noise}

The selected $70 \mathrm{kHz}$ switching power supply generates a ripple noise of $0.03 \%$, i.e., $46 \mathrm{Vrms}$ at $128 \mathrm{kV}$. The sense wires plus front-end electronics suffer their greatest sensitivity to noise in the nearby frequency range from $100 \mathrm{kHz}$ to $10 \mathrm{MHz}$, implying the need for a power supply noise filter. Voltage noise on the cathode can also inject current capacitively into the sensing wires, although the internal grid attenuates this contribution.

The power supply noise filter consists of a two-stage passive external low pass RC filter, with the capacitance provided by HV cable and the TPC cathode, and with resistors installed inside oil filled "surge boxes" provided by the Tevatron separator system. The filter resistors, with the available cable capacitance, must provide a sufficiently low bandwidth cutoff for the low pass filter, without creating an unacceptable voltage drop from the HV power supply to the TPC. A 4 $\mathrm{kV}$ drop across the filter resistors can be tolerated.

The resistance used in the noise filters is proportional to the resistance of the voltage divider chain. A divider current of no greater than $8 \mu \mathrm{A}$ is acceptable. (This current is much larger than the total cosmic ray current of $0.8 \mathrm{nA}$.) A two-stage filter, using $250 \mathrm{M} \Omega$ resistors and $6 \mathrm{~m}$ of HV cable in each stage, cuts the power supply ripple contribution into the wire amplifiers to 15 electrons, using very conservative assumptions (a more detailed analysis remains under way). With the choice of $8 \mu \mathrm{A}$, the divider chain uses four parallel resistors of $1000 \mathrm{M} \Omega$ each at each stage. 


\section{Light Collection System (WBS 1.8)}

\subsection{Overview}

This chapter details the technical design of the light collection system for MicroBooNE. Subsequent sections describe photon production in liquid argon (LAr), photomultiplier tubes (PMTs) and bases, wavelength-shifting (WLS) plates, the PMT unit configuration, mounting and support structures, cabling and the cryostat feedthrough, and optical simulations.

MicroBooNE physics goals require the light collection system to trigger on $40 \mathrm{MeV}$ kinetic energy protons produced in neutrino interactions, and, if possible, on $5 \mathrm{MeV}$ electrons, associated with interactions caused neutrinos produced in nearby supernovae.

Primary design and assembly the light collection system rests with MIT, with collaborative contributions from Fermilab, Princeton, and Saint Mary's University of Minnesota.

\subsection{Photon Production in Liquid Argon}

Charged particles passing through the LAr produce both scintillation and Cherenkov photons, with the isotropically emitted scintillation light dominating by a factor of five. Both sources can be detected within the detector, but the MicroBooNE light collection design optimizes the measurement of the scintillation light.

An $\mathrm{MeV}$ of energy loss by a minimum-ionization particle (MIP) results in the production of approximately 24,000 scintillation photons with wavelength $\lambda \approx 128 \mathrm{~nm}$ via excitation or ionization of argon atoms and their formation of excited Ar dimer states. Dimers produced via the excitation process emit a prompt component of the light with lifetime $\tau \approx 6 \mathrm{~ns}$. Those formed via ionization emit a slower component with $\tau \approx 1.6 \mu \mathrm{s} .{ }^{1}$ The prompt component, representing about $25 \%$ of the light, proves more useful for triggering.

MicroBooNE can make good use of prompt scintillation photons. Requiring light to be intime with the Booster Neutrino Beam pulse enables a beam trigger that allows for substantial reduction of the amount of data written to storage. The prompt and fast light pulse provides a TPC start time (T0) for non-beam-related events such as those initiated by supernova neutrinos. The amount of light (total PMT charge) can also be used as a trigger for different types of events. Finally, the spatial centroid of the light can feed into the offline software to provide useful starting coordinates for a neutrino interaction vertex.

\subsection{PMTs}

\subsubsection{Choice of Photomultiplier Tubes}

MicroBooNE uses thirty 8-inch diameter PMTs (bi-alkali 14-stage Hamamatsu R5912$02 \mathrm{MOD})$ that provide $0.85 \%$ photocathode coverage over the surface of the TPC. 
Figure 9.1 summarizes characteristics of these PMTs. ${ }^{2}$ Eight inch diameter PMTs provide the most cost-effective photocathode coverage for the experiment. A platinum under-layer on these PMTs allows them to operate at temperatures below $150 \mathrm{~K}$. These devices give a highquality signal, with gains of order $10^{9}$ well over the $10^{6}$ needed to provide input for the readout electronics. By decreasing PMT operating voltage from the nominal $1700 \mathrm{~V}$, high voltage breakdown risks at the feedthroughs and in the LAr can be reduced while maintaining gains above $10^{7}$.

IR5912, R5912-02
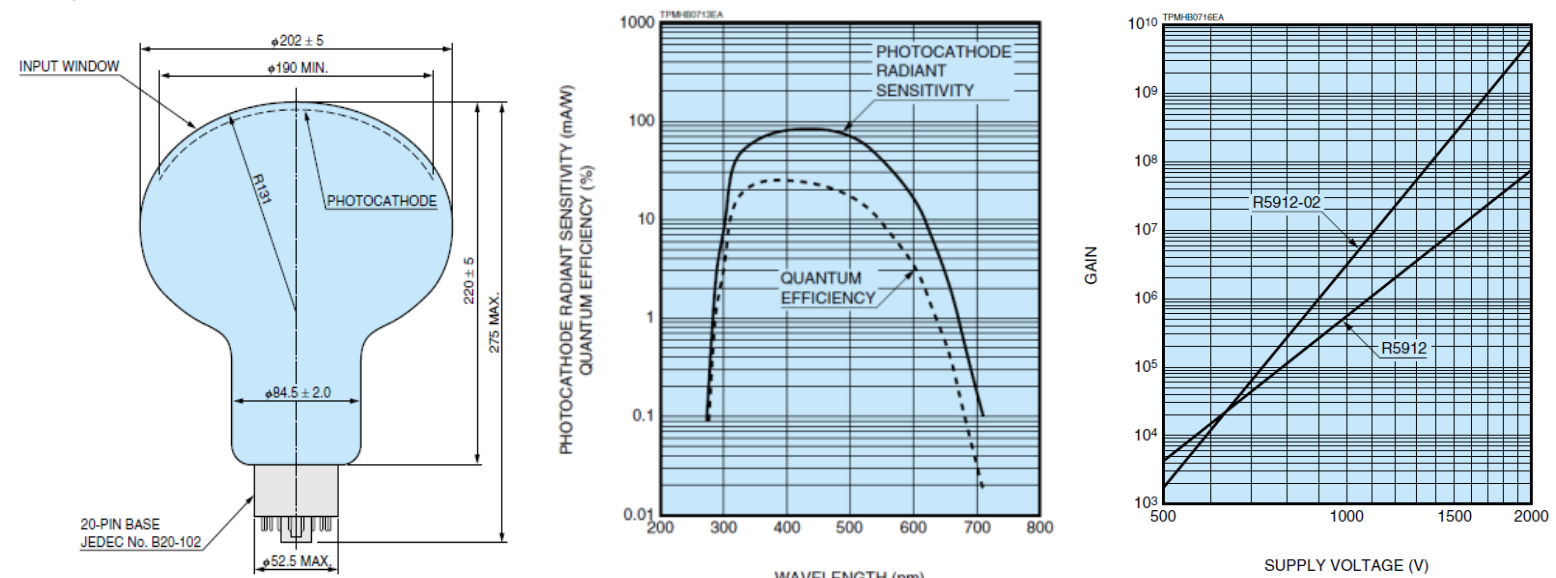

Figure 9.1 R5912-02 Hamamatsu 8-inch PMT drawing (left), quantum efficiency (middle), and gain curve (right).

\subsubsection{PMT Efficiency}

The "global quantum efficiency" (GQE) characterizes overall PMT unit response. The GQE includes VUV-to-visible photon conversion efficiency of the wavelength shifter (Section 9.4), geometric efficiency of visible photons captured by the PMT glass bulb, visible-photon transmittance of the glass window and the $\mathrm{Pt}$ under-layer, quantum efficiency of the photocathode, photoelectron-collection efficiency of the electric field applied between the photocathode and the first dynode, and the overall effect of operating at cryogenic temperature. The VUV-to-visible conversion efficiency and the quantum efficiency of the photocathode dominate the GQE. The ICARUS experiment obtained a final GQE of about $6 \%$ across the entire tube and $8 \%$ for the photons entering the tube at normal

incidence. ${ }^{1}$ MicroBooNE plans to measure the GQE for a few units before installation. Absolute GQE measurements should be possible after installation via cosmic rays. The TPC wire planes and the TPC ground plane mesh block $27 \%$ of light. Taking this into account, roughly two photoelectrons should be detected per $1 \mathrm{MeV}$ energy loss in the LAr by a MIP.

\subsubsection{PMT Base Design}

The PMT front-end signal processing consists of two components: a flat PC-board-style PMT base that fits onto each tube inside the cryostat, and a signal splitter mounted outside 
the cryostat. The signal splitter separates signal and HV, both of which use the same cable. This minimizes the number of cryogenic feedthroughs and the cables' outgassing into the LAr. A $10 \mathrm{nF}$ capacitor in the signal splitter decouples signal and DC HV. Figure 9.2 shows a photograph of the actual PMT base, and Figure 9.3 shows the PMT base and splitter schematics.
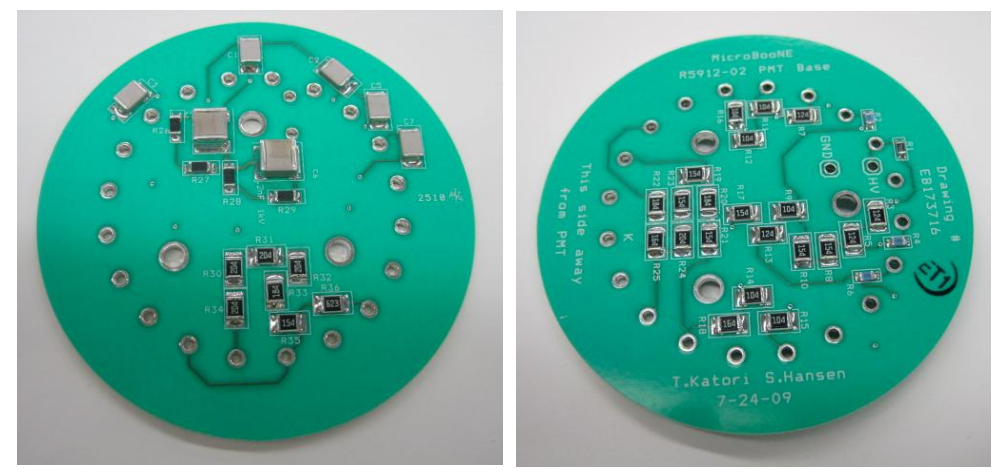

Figure 9.2: Top and bottom views of PMT base (2.4-inch).
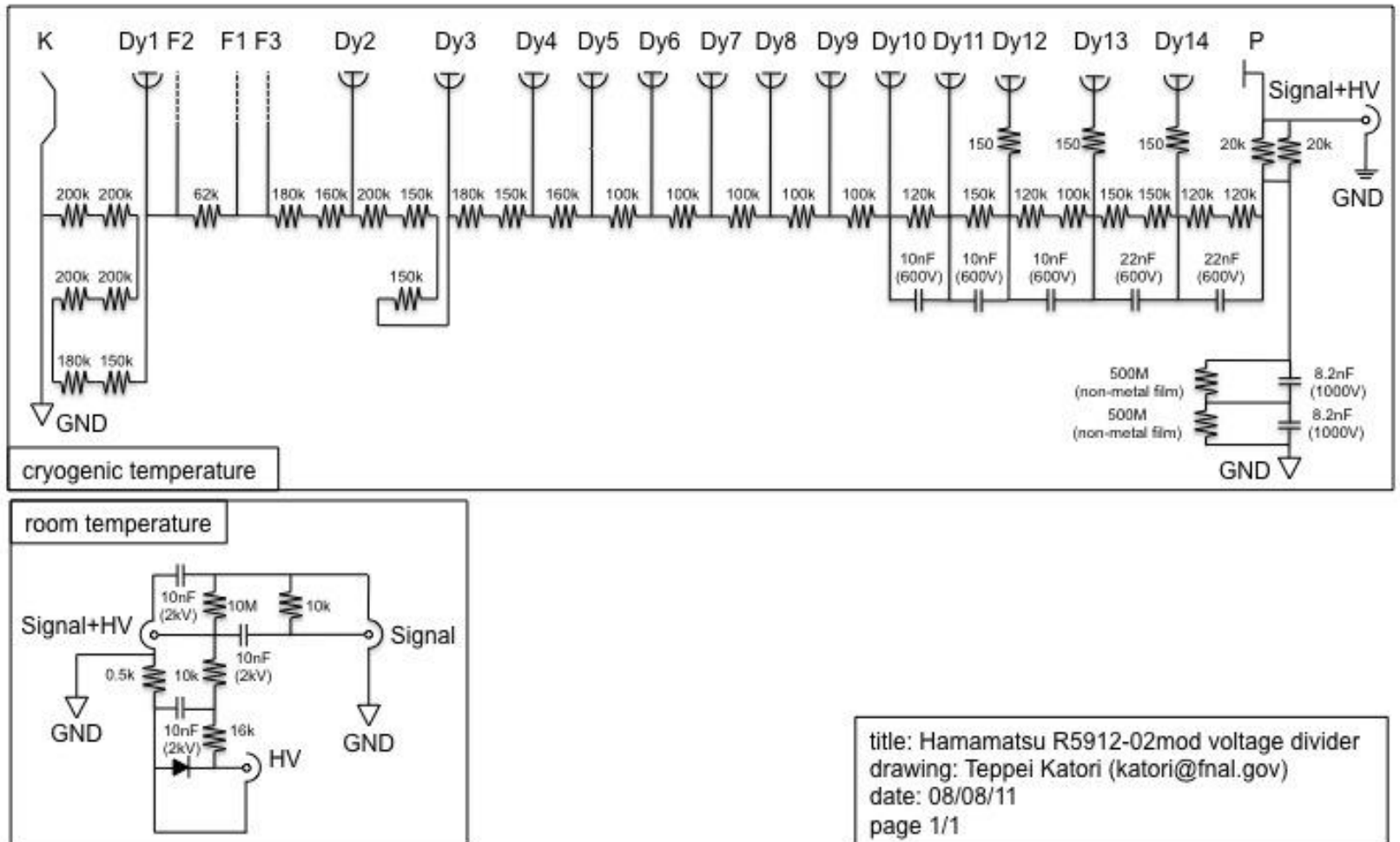

title: Hamamatsu R5912-02mod voltage divider drawing: Teppei Katori (katori@fnal.gov) date: $08 / 08 / 11$

page $1 / 1$

Figure 9.3 PMT base and splitter schematics.

The PC board is made from Rogers RO4000-series woven glass-reinforced laminates, rather 
than the conventional FR-4 fiberglass. This material offers the advantages of lower water contamination, which helps to maintain high LAr purity, and a smaller thermal expansion coefficient, which lessens mechanical stresses on installed passive components as the PC board cools to cryogenic temperatures. The passive components include only metal film resistors and COG/NP0 capacitors, both of which perform well at cryogenic temperatures. All materials pass purity tests in the Fermilab materials test stand (MTS). A commercial vendor produces the PC boards, and Fermilab EED technicians stuff them with the passive components. MicroBooNE PMT members then solder the boards onto the wire outputs of the phototubes. Each base solders to a position approximately $1 \mathrm{~cm}$ from the bottom of its PMT, the closest possible distance at which it can be safely attached to the PMT vacuum seal tip. The high voltage (HV) and ground (GND) lines from each primary cable attach to the base of each PMT through pin receptacles.

\subsection{Wavelength-Shifting Plates}

\subsubsection{Choice of Wavelength Shifter}

The $\lambda=128 \mathrm{~nm}$ scintillation light in LAr must be shifted to the visible in order to be detected by the bi-alkali phototubes. A tetraphenyl butadiene (TPB) layer acting as a wavelength shifter performs this task in MicroBooNE. The TPB absorbs in the UV and emits in the visible with a peak emission at $\lambda=425 \pm 20 \mathrm{~nm}$, a favorable wavelength for detection by PMTs. The WLS emits an average of 1.2 visible photons for every 128 -nm photon absorbed. ${ }^{3}$

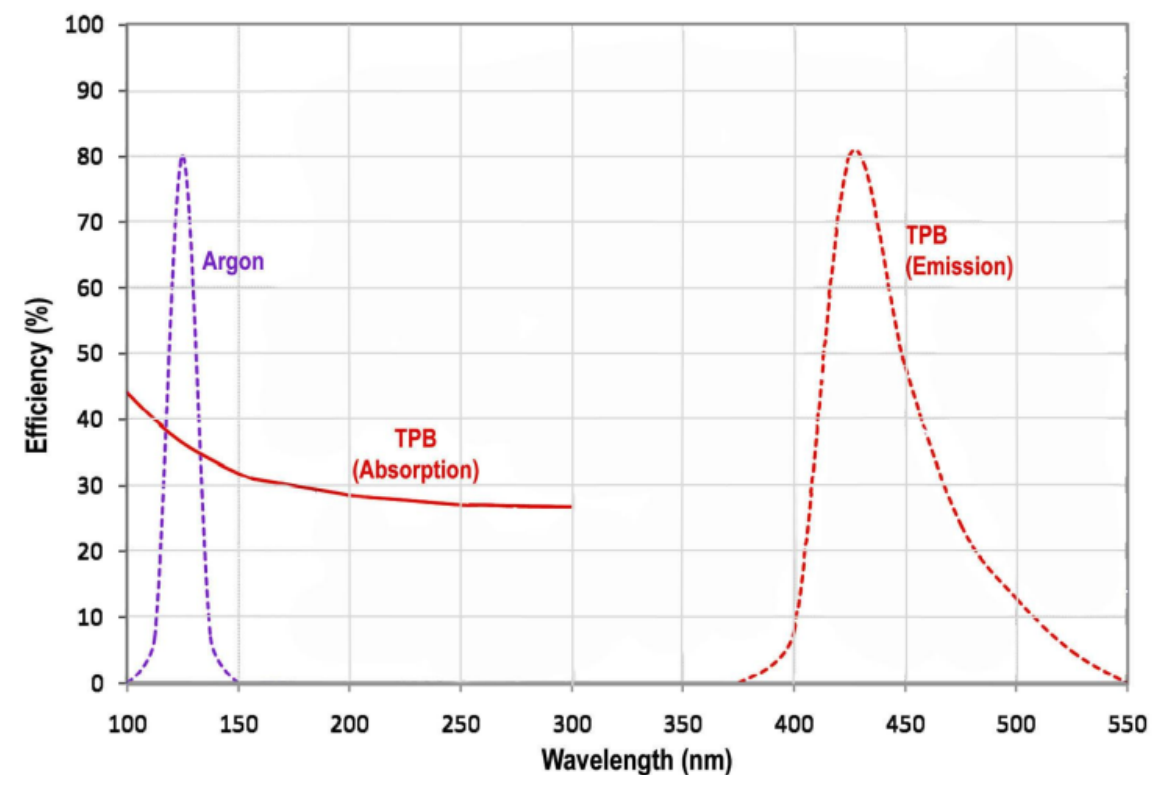

Figure 9.4 TPB layer emission (arbitrary units) and absorption spectra in the high UV.

Figure 9.4 shows a TPB layer absorption curve. The flatness of the absorption curve across a 
wide range of wavelengths in the high UV makes TPB a very versatile wavelength shifter for UV detectors. As a result, it is widely used and available for a reasonable price $(\$ 100 / 1 \mathrm{~g})$. Figure 9.4 also shows the positions of the argon scintillation peak and the TPB emission peak, both in arbitrary units.

The WLS design incorporates scintillation grade (>99.9\% pure) TPB wavelength shifter on coated acrylic plates mounted above the PMTs. These plates have 1/16-inch $(0.16 \mathrm{~cm})$ thickness and a 12 inch $(30.5 \mathrm{~cm})$ diameter, with the TPB coating on the side of the plate facing into the active volume of the detector.

Quality assurance considerations favor coating the WLS plates rather than the PMTs. The increase in collection efficiency achieved by a wider plate compensates for the decreased solid angle acceptance of the phototube resulting from the angular distribution of re-emitted light from the plate. Acrylic has the advantages of ease of machining and a coefficient of thermal expansion that matches that of polystyrene (PS), applied to the acrylic to form a matrix that mechanically stabilizes the TPB.

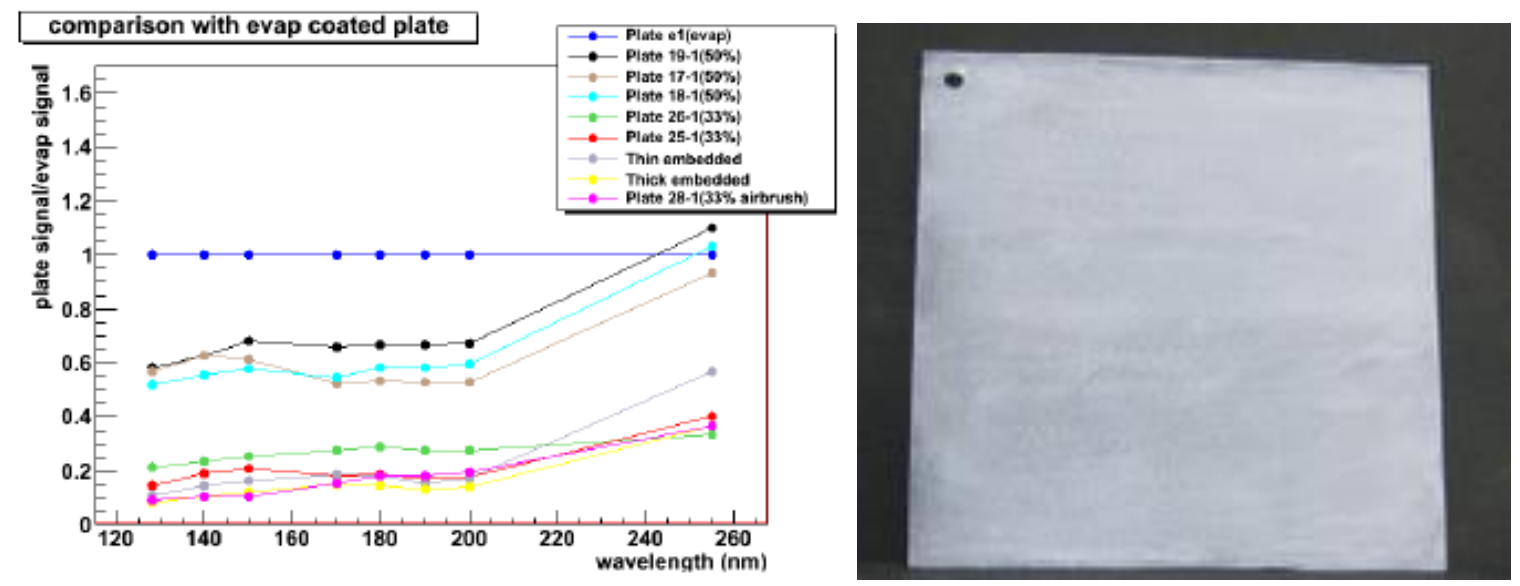

Figure 9.5: Results of vacuum monochrometer data, normalized to the performance of the evaporatively coated plate, used to select the optimal TPB coating method (left). Sample TPB-PS coated plate (right).

\subsubsection{Method of Coating}

The TPB-PS solvent is dissolved in toluene solution in a fume hood. The procedure consists of dissolving $1.0 \mathrm{~g}$ of TPB in $50 \mathrm{~mL}$ of toluene, adding $1.0 \mathrm{~g}$ of scintillator-grade PS pellets to the toluene, and then agitating the mixture until the PS dissolves. The mixture is brushed on to clean acrylic plates. A few drops of ethanol are then brushed on to produce a more uniform coating during drying.

In order to achieve a more uniform coating, the painting procedure repeats three times per plate, with time to dry added between each repetition. Figure 9.5 shows a sample TPB-coated plate using this process. 
A series of light emission tests on plates produced with several coating techniques validate the decided-upon method. These tests use a vacuum monochrometer with a deuterium lamp, as shown in Figure 9.5. Figure 9.5 shows that the vacuum evaporated plate with a TPB surface density of $\sim 0.2 \mathrm{mg} / \mathrm{cm}^{2}$ produces the highest light response. The TPB-PS coating with 50\% TPB mass concentration has 50-60\% of the efficiency of a vacuum evaporated plate, but it has advantages in terms of cost, durability, and ease of production in the lab. Using this coating method still leaves more light than that needed for triggering on a $5 \mathrm{MeV}$ electron.

\subsubsection{Production and Storage}

Wavelength-shifting plates can be produced quickly at low cost. Plate testing involves light absorption and reemission measurements at 18 positions using a $\lambda=255$ $\mathrm{nm}$ commercially available LED. These tests can easily be performed at the rate of a few plates per day. MicroBooNE requires 30 plates; 60 will be produced to allow installation of those with the best measured response.

Humidity and light exposure can degrade TPB and reduce light output from coatings. ${ }^{4}$ Each WLS plate will be stored in gaseous argon and in the dark until installation. Thermal annealing and vacuum storage methods currently being tested may be used as well.

\subsection{PMT Unit Configuration}

Each PMT unit consists of an 8-inch PMT mounted between three cylindrical polyether ether ketone (PEEK) posts on a perforated stainless steel back-plate, with a WLS coated plate on the TPC side of the PMT, and an electronic base at the other end with a primary cable attached. Figure 9.6 shows a 3-D drawing and mechanical model of a PMT unit. Each PEEK post has a carved-out curvature to cradle the waist of the tube. ${ }^{1}$ Its ease of manufacture, small coefficient of thermal expansion, and low water contamination make PEEK is an attractive material despite its price. A 1 inch $(2.54$ $\mathrm{cm})$ diameter, $1 \mathrm{ft}(30.5 \mathrm{~cm})$ long rod costs $\sim \$ 60$. The perforated back-plate allows flexibility in positioning the PEEK posts and in mounting the unit on the support rack. The perforations in the back-plate also enable convection currents in the LAr to take away the small amount of heat generated by the PMT base ( $<1 \mathrm{~W}$ per base). All elements of a PMT unit are cleaned and readied for assembly immediately prior to mounting on the support rack at the time of installation. 


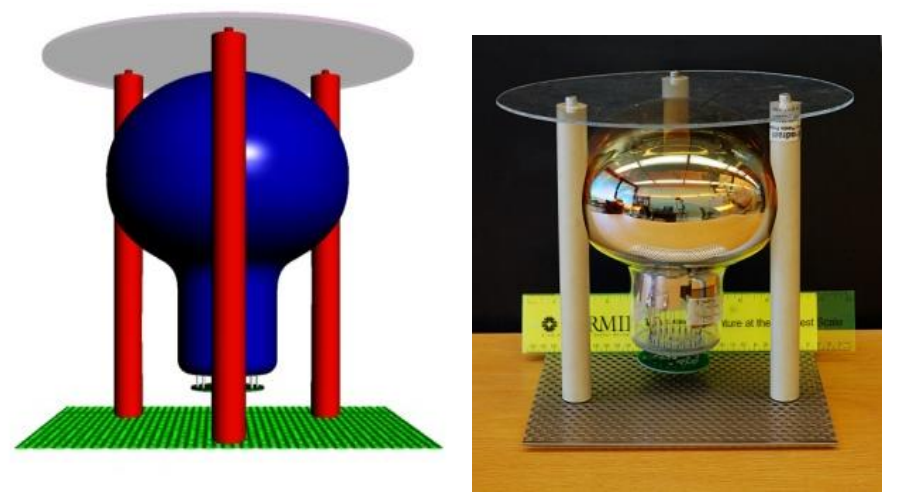

Figure 9.6 3-D drawing (left) and Mechanical model (right) of a PMT light collection unit.

\subsection{Mounting and Support Structure}

\subsubsection{Mounting of PMT Units}

The PMT units' perforated stainless steel back-plates of the PMT units affix to a stainless steel support rack that slides into the cryostat along the side wall behind the wireplane side of the TPC, as illustrated in Figure 9.7. The rack aligns the PMTs in a plane looking straight into the cryostat. Optical simulations (Section 9.8) verify that this mounting scheme optimizes PMT performance.
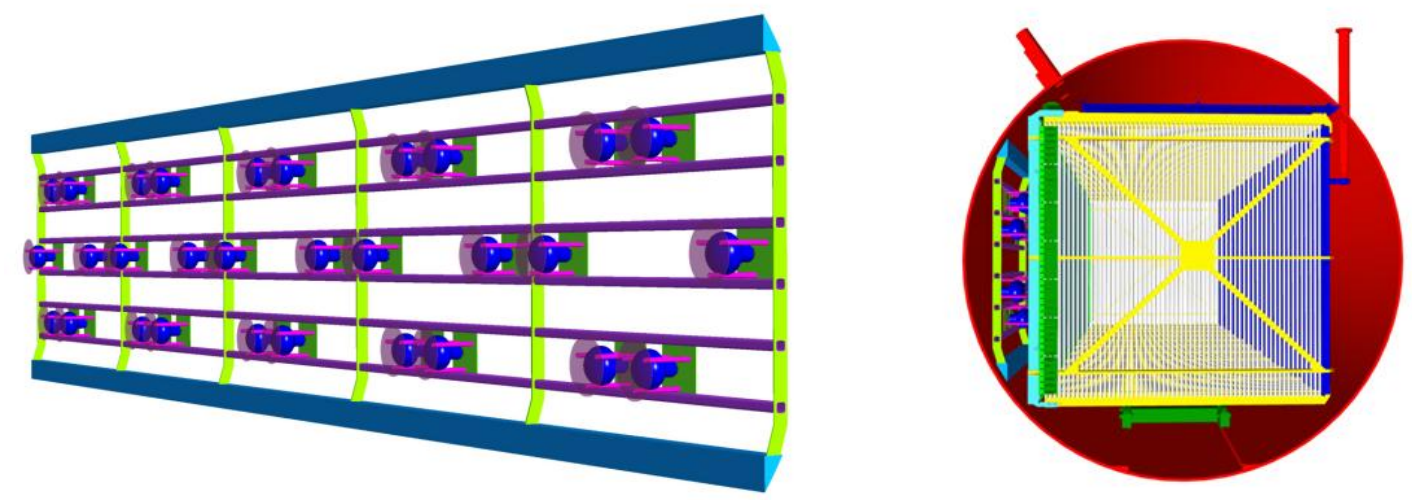

Figure 9.7: Model of support rack with PMT units mounted (left); final position of installed PMT Support rack inside the cryostat, viewed from removable-head end (right).

Units mount in five rows arranged so that the cross bars of the TPC do not obstruct any PMT. The rack design ensures that no PMTs touch the TPC itself. Figure 9.7 shows 30 units mounted to the rack, the baseline design for MicroBooNE. The rack can accommodate an additional 40 units. The detailed mounting scheme of the PMT unit to the rack remains under investigation, with pin or rivet connections possible.

\subsubsection{Support Rack Design}

Five stainless steel components, or slices, of vertical height 72 inches $(1.83 \mathrm{~m})$ and horizontal length 81.6 inches $(2.07 \mathrm{~m})$ comprise the rack, giving it a total horizontal length of 408 inches $(10.36 \mathrm{~m})$. Figure 9.8 shows a diagram of one such rack slice, as well as the total horizontal 
configuration of all five components. Six PMT units mount to each slice.
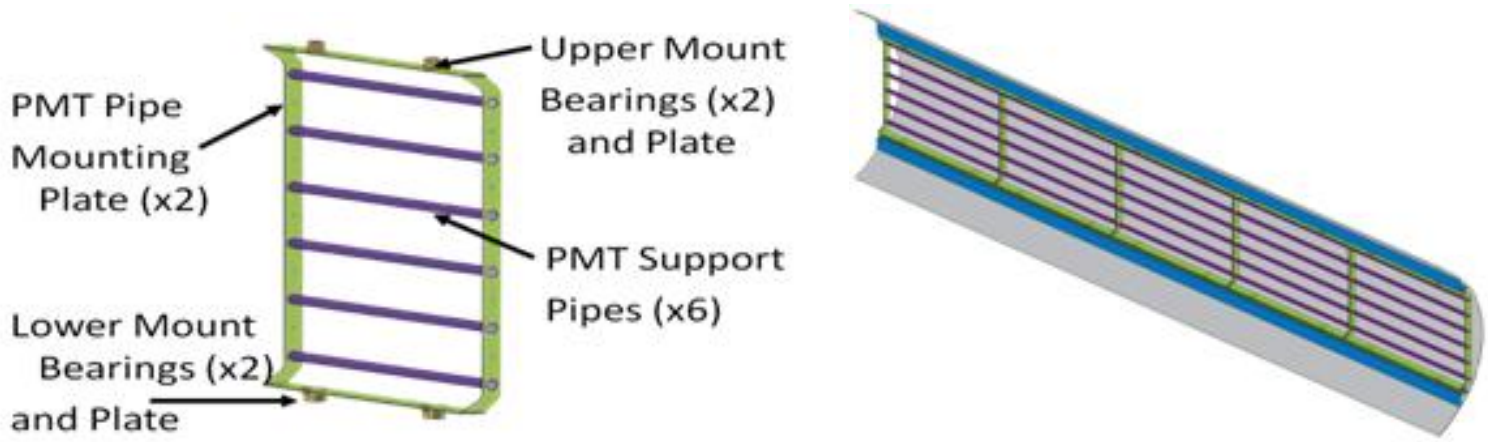

Figure 9.8: A model of one of five identical rack "slices" (left) and a model of the entire PMT support rack oriented against the cryostat wall (right).

Thomson bearings fitted to the lower edge allow each slice to slide into the cryostat on rails previously mounted in the vessel. Figure 9.9 shows the configuration of these bearings on a rack slice.
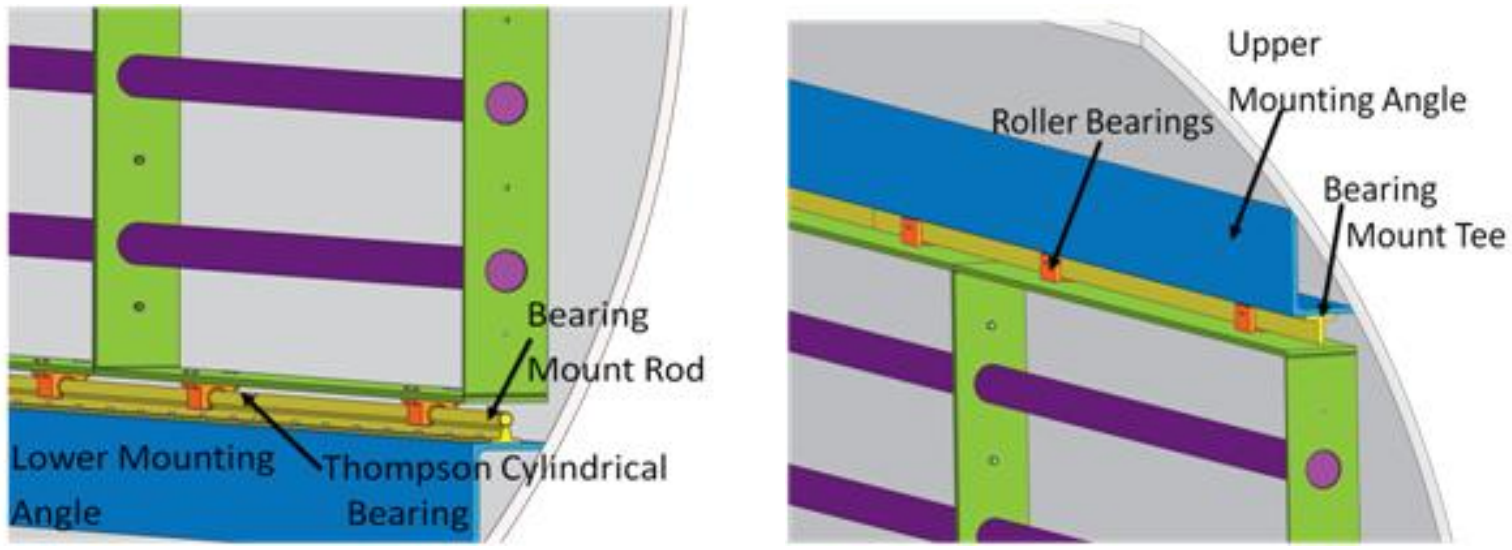

Figure 9.9: The upper and lower details of the PMT rack design.

\subsubsection{Installation of PMT-Loaded Support Rack}

The division of the rack into five equal vertical slices allows easy installation. Each slice contains six horizontal tubes, on which the PMT back-plates mount, held together with two vertical supports and encased by a top and bottom rail. The PMT units are attached to the rack by sliding their stainless steel back-plate behind the tubes and fastening them in place. The holes are drilled in the horizontal tubes of the rack before the rack enters the clean room.

A temporary mounting rail outside the cryostat holds each slice during the 
attachment of the PMT units. The fully outfitted slice then slides into the cryostat on rails located at the top and bottom. The top and bottom rails slide onto upper and lower rail guides (Thomson rods) mounted to brackets welded to the interior of the cryostat wall prior to purity testing and certification of the vessel. Thomson bearings on the bottom rail of the support $\mathrm{rack}$ allow the slices to slide easily along the Thomson rods.

The PMT support rack will be the final component of the MicroBooNE detector to move into the cryostat before closure. This allows the WLS plates to be stored in a dry, inert environment for as long as possible. The cabling from each PMT routs to a patch panel attached to the back of the support rack nearest to the open end of the cryostat. The fiveslice scheme permits easy tracking and handling of the cables from all PMT units. After each slice of support rack is installed, a check of the PMT pulses of each set of six PMTs will be performed for final quality assurance.

\subsection{Cabling and Cryostat Feedthrough}

\subsubsection{Three-Part C a b ling Design}

The PMT cable system delivers HV from the signal splitter to the PMTs and signals from the PMTs to the signal splitters. It divides into three parts: primary, intermediate, and external. Primary cables solder to the PMT bases at one end and to an SHV female connector at the other. These connectors feed into a patch panel mounted at the end of the support rack on the rack slice closest to the open end of the vessel, as mentioned above. This allows continued access to the patch panel after the entire rack slides into the cryostat. Intermediate cables run from the patch panel to the PMT feedthrough flange. Each intermediate cable has an SHV male connector at the patch panel end and attach to the bottom of the feedthrough. External cables run from the outside of the feedthrough flange to the splitter boxes located outside of the cryostat.

This three-part design allows for easy installation. The intermediate cables install first while the cryostat is empty. These get pulled aside and placed in such a way that they can be accessed from the entrance end of the cryostat. The TPC installation occurs next. Then each fully instrumented and cabled rack slice slides into the cryostat with its primary cables held aside. Upon insertion of all five rack slices in the vessel, the primary and intermediate cables attach to the patch panel.

\subsubsection{Choice of Cables and Connectors for Use in LAr}

The primary cables, patch panel, and intermediate cables all remain immersed in LAr during detector operations. MicroBooNE only employs components used in previous LAr experiments at Fermilab. In addition, all materials pass through the MTS for purity testing.

The light collection system uses RG180 cable. This Teflon-coated, 0.14 inch $(0.36 \mathrm{~cm})$ diameter cable has high impedance (95 ohms) to match with the pre-amplifier and low 
capacitance $(6.8 \mathrm{pF} / \mathrm{m})$ to preserve small signals. The cable carries an AC voltage rating of $1100 \mathrm{~V}$; tests show its DC rating to be at least three times higher. The RG180 cables employ standard SHV connectors used on RG58 cables. The SHV connector impedance has a negligible effect on the 20-30 ns PMT signals.

Fermilab PPD technicians have developed all PMT cable production procedures, and they will perform all cable production tasks. This includes cutting slits cut at one foot $(30 \mathrm{~cm})$ intervals along the cable outer jackets to accelerate outgassing, and applying shrink tubing to increases the cable thickness of the thinner RG180 to make a good fit to the RG58 connectors.

\subsubsection{Cryostat Feedthrough Flange}

The intermediate cables, which terminate at one end with standard SHV connectors, connect to a 14-inch $(35.6 \mathrm{~cm})$ CF feedthrough located above the PMT patch panel on the top of the cryostat. The cables run up through a 6 -inch $(15.2 \mathrm{~cm})$ diameter chimney at nozzle 1 of the cryostat, closest to the removable head end of the flange. The top of the chimney will meet the CF flange as shown in Figure 9.10.

The connectors and portions of cable exposed to gaseous LAr must be protected in order to avoid high voltage breakdown. The scheme developed by technicians at Fermilab PPD and shown in Figure 9.10 provides this protection. In order to prevent sparks between the HV pin and the grounded flange, G10 tubes cover the inner line. Inner conductors meet pins in the G10 tubes, and separated outer conductors attach to a flange.
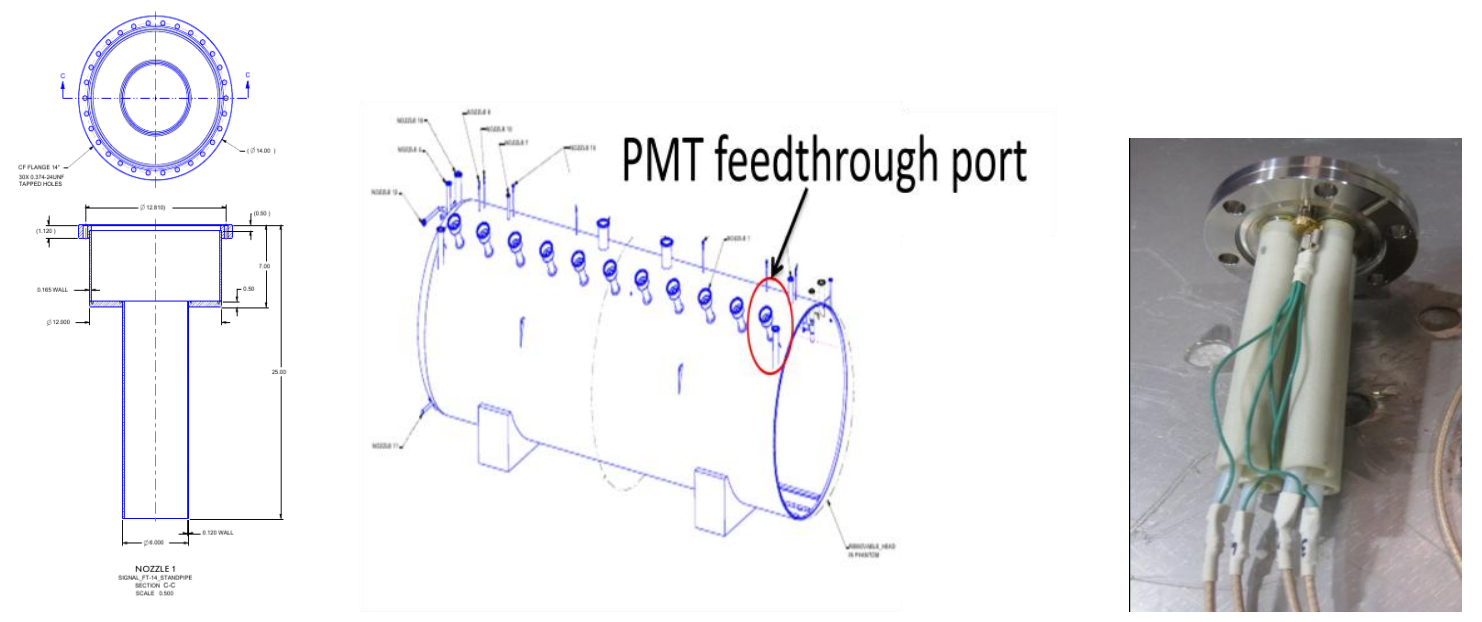

Figure 9.10: Diagram of the PMT feedthrough chimney (left); location of the PMT feedthrough port on the cryostat (center); prototype of HV breakdown-protected feed-through with four SHV ports (right).

Outside the cryostat, RG180 cable with standard SHV connectors make up the external cables. These cables connect to signal splitters placed in the PMT rack on the platform above the cryostat. This marks the point where responsibility passes from the PMT group to the electronics 
group. Chapter 10 describes routing of the HV cables into the splitter box and the signal cables out of the splitter box to the PMT shaper electronics.

\subsection{Optical Simulations}

Simulation work of the light collection system using the LArSoft software package supports many of the design decisions described in this chapter. A summary of these studies follows. Chapter 12 provides more details of the LArSoft simulation tools.

The trigger sensitivity study over the fiducial volume involves using the full optical simulation of the response to $5 \mathrm{MeV}$ scintillation-equivalent light sources placed at each point in the $x=0$ and $y=0$ planes, as shown in Figure 9.11. The study assumes a total optical assembly efficiency of 0.03. All point sources predict production of at least ten photoelectrons for $5 \mathrm{MeV}$ scintillation equivalent point sources placed on the $x=0$ or $y=0$ planes. The study therefore shows that the PMT system can trigger on $5 \mathrm{MeV}$ scintillation-equivalent light over the TPC volume, with an assumption of 0.03 efficiency.
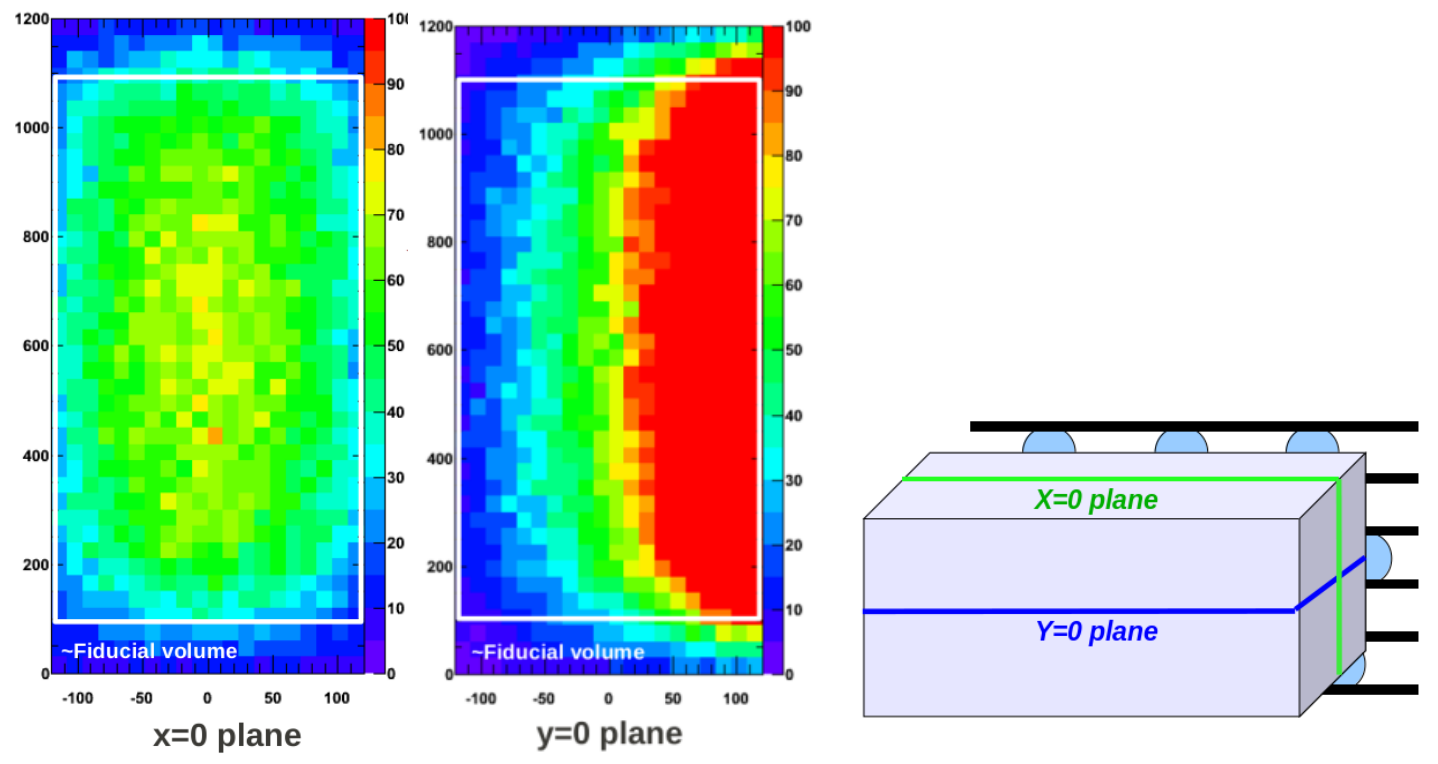

Figure 9.11 Trigger sensitivity in the $x=0$ and $y=0$ planes.

A second study examining the redundancy of the system involved checking the total PMT response with one PMT removed from the system and each PMT removed in turn. The study shows no significant degradation in trigger capability due to the loss of one PMT.

A third study considered two candidates for the PMT support rack, one straight and one curved, as shown in Figure 9.12. Full optical simulations of the performance of each rack show that low light yield is not a problem for either design. The straight rack gives a more uniform total PMT response over the fiducial volume. The curved rack predicts a higher response for points near the cryostat central axis, and a lower response for off-axis points. This study thus substantiates the choice of the straight rack design. 

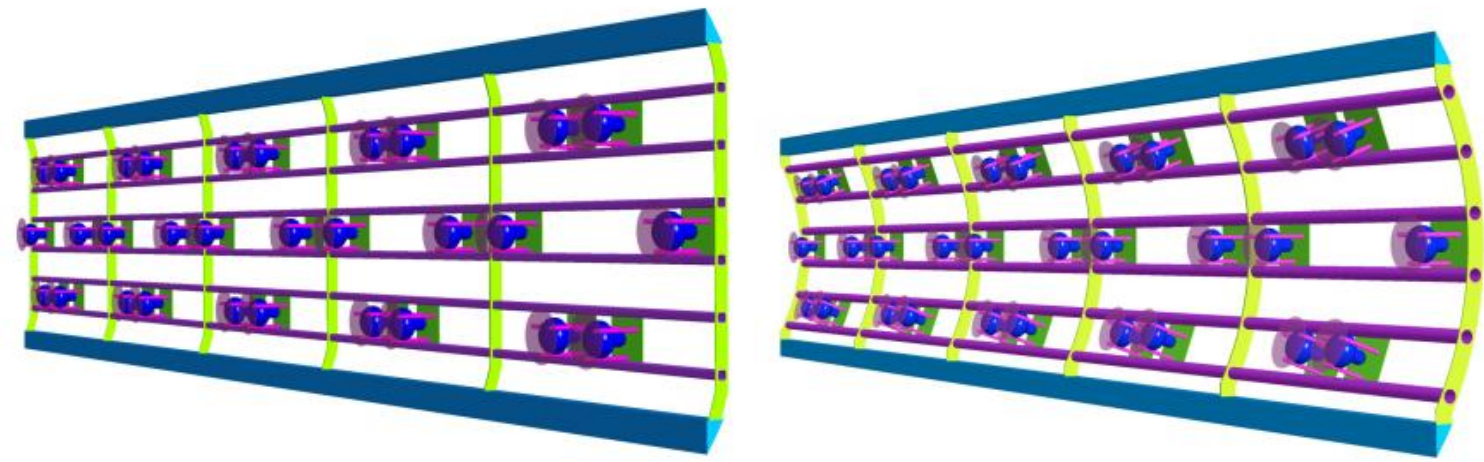

Figure 9.12: Straight (left) and curved (right) support rack design alternatives.

A final study analyzes the path of photons on a track-by-track basis for several events in the simulation to aid in the understanding of the optical physics processes most relevant to MicroBooNE events. Figure 9.12 shows an example. A neutrino event generated with the GENIE neutrino interaction generator in the upper left corner of the cryostat produces scintillation photons. Each photon scatters an average of 0.76 times, undergoing an average of 0.19 reflections. The simulation agrees well with studies by ICARUS $^{1}$, lending confidence to the validity of the MicroBooNE tools.
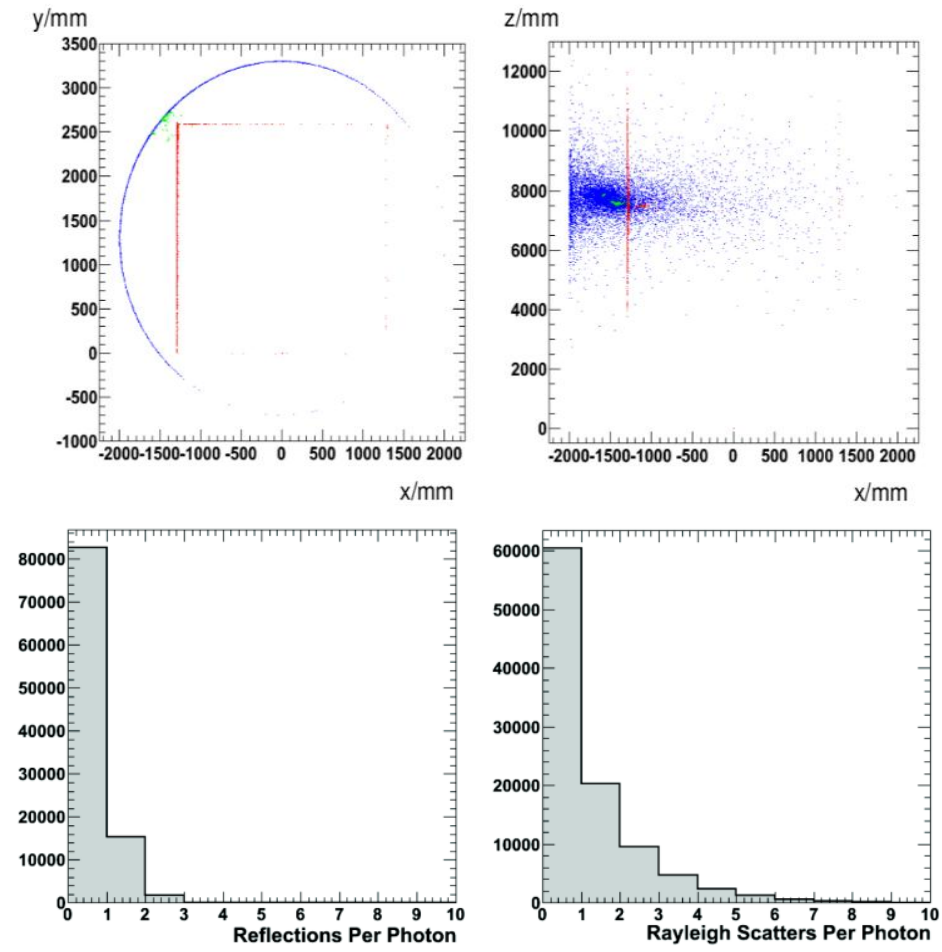

Figure 9.13: Photon maps (top) and interaction statistics (bottom) for a typical GENIE generated neutrino interaction event. Green points show photon production vertices. Blue points show absorptions at boundaries with known reflectivity properties. Red points show absorptions at other boundaries, which are assumed black. The bottom plots show the number of reflections and scatters per photon. 


\footnotetext{
${ }^{1} \mathrm{M}$. Antonello et al., Analysis of Liquid Argon Scintillation Light Signals with the ICARUS T600 Detector, Tech. Rep. ICARUS-TM/06-03 (2006)

${ }^{2}$ Hamamatsu Photonics. http://sales.hamamatsu.com/assets/pdf/parts R/LARGE AREA PMT TPMH1286E05.pdf ${ }^{3}$ arXiv: 1104.3259v1 [astro-ph.IM]

${ }^{4}$ R. Acciarri et al., "The WArP Programme,"

http://www.Ings.infn.it/Ings infn/contents/Ings en/research/experiments scientific info/library publications/pre print reports/report08/LNGS-AR-2008-WArP.pdf (2008)
} 


\section{Readout and Data Acquisition (WBS 1.5 and WBS 1.9)}

\subsection{Overview}

The MicroBooNE readout electronics system (WBS 1.5) includes two subsystems, the TPC readout electronics and the PMT readout electronics. The TPC readout electronics system reads out, digitizes, and processes the detector signals from the TPC induction and collection wires. The PMT readout electronics system reads out, digitizes, and processes the PMT signals. The PMT readout electronics system can produce trigger signals for the whole readout and data acquisition (DAQ) system. The TPC and PMT readout electronics systems have different analog front end and digitization designs, but they share the same back end design that organizes and packages the data, and then sends it to the DAQ system. The DAQ system (WBS 1.9) concentrates the data from both TPC and PMT readout electronics systems, providing the buffer for data before the event is built and recorded. The readout and DAQ can function together in a variety of triggered or trigger-less modes. The DAQ system also provides additional functionalities for run control, monitoring, system operations and alarms.

\subsection{TPC Readout Electronics}

\subsubsection{Introduction}

The TPC readout electronics system must process signals from 8,256 detection wires in the chamber. It includes the cold electronics, warm interface electronics, digitizing and data handling electronics, cabling, and signal feedthroughs. The readout channels originate from two induction planes, denoted $\mathrm{U}$ and $\mathrm{V}$, with wires running at $\pm 30^{\circ}$ with respect to the beam direction, which defines the z-axis, and one collection plane (Y-plane) with vertical wires perpendicular to the beam.

Figure 10.1 outlines the block diagram of a single TPC readout channel. A CMOS analog front end ASIC inside the cryostat (the "cold electronics") pre-amplifies and shapes the signal before sending it over a $2 \mathrm{~m}$ long twisted pair cable through a feedthrough to the intermediate amplifier installed outside the cryostat within a Faraday cage on top of the signal feedthrough (the "warm interface electronics"). The intermediate amplifier amplifies the signal further to make it suitable for transmission over a $20 \mathrm{~m}$ long cable to the TPC readout board ("digitizing and data handling electronics") housed in a crate. The TPC readout board continuously samples received signals, passing the digitized outputs from the ADC to an FPGA for data processing, reduction, and storage. The FPGA buffers processed data on board temporarily and then transmits it to DAQ PCs through the crate backplane and optical links. The PCs store data on hard drives for further analyses. 


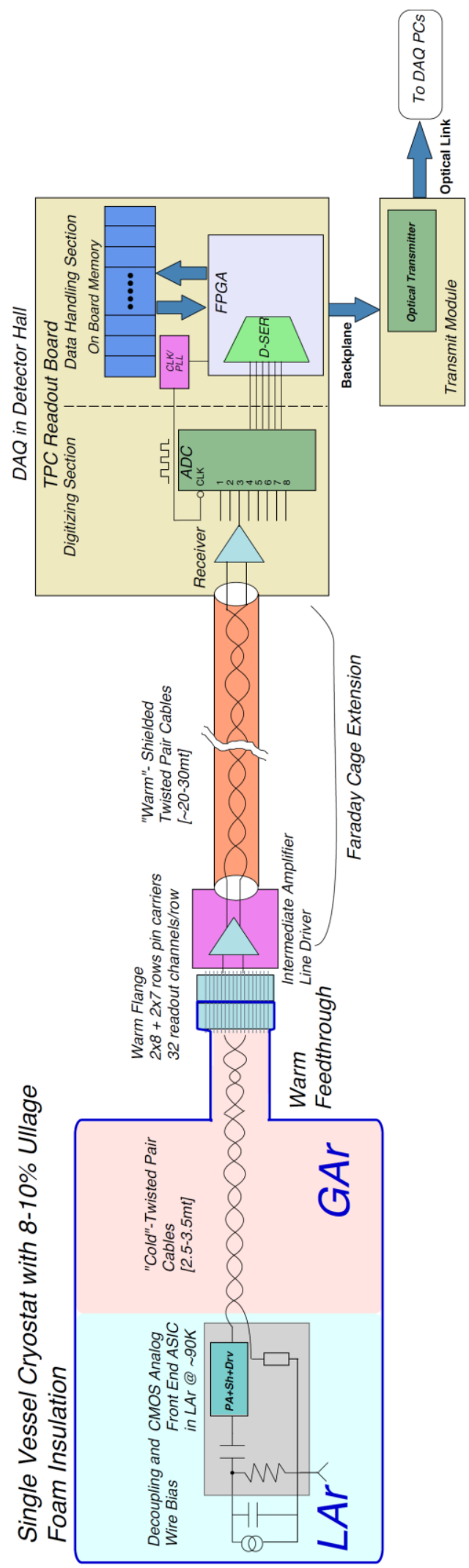

Figure 10.1 Information flow of a single TPC readout channel. 


\subsubsection{Technical Design Criteria}

The TPC readout electronics system operates in two modes: "accelerator" and "supernova." Accelerator events derive from neutrino beam triggers that occur at the maximum Fermilab Booster repetition rate of $15 \mathrm{~Hz}$ (with $10 \mathrm{~Hz}$ being the current operational mode); no loss of information must be allowed for data recorded in this mode of operation. A PMT trigger in coincidence with the Booster beam gate may be used to reduce the data taking rate. Lossless data compaction with, for example, Huffman coding may be used to reduce data size requirements. On the other hand, potential supernova events, for which MicroBooNE has no triggering capability, force the data to be stored until an external supernova alert is received, which may require on the order of one hour. Some data reduction through, for example, decimation, must be allowed for supernova candidates.

The main design criteria of the TPC readout follow:

- Preamplifier noise: Equivalent noise charge ENC < 660e. Noise must be kept as low as possible to allow the detection of minimum ionizing particle (MIP) tracks that drift the maximum distance to the induction wire planes.

- Shaper peaking time: $1.0 \mu$ s. Detector signal response simulations (MicroBooNE DocDB 257 ) indicate an optimal shaper peaking time of $1.0 \mu$ s for both collection and induction wire planes. Drift electron diffusion smoothes out higher frequency components of the response that include no useful information.

- ADC dynamics range: 12-bit. The requirement to observe in the induction plane a MIP produced at the far end of the TPC determines the lower end of the dynamic. The need to collect charge from a highly ionizing stopping proton without suffering saturation effects sets the upper end. Monte Carlo simulations (MicroBooNE DocDB 167) verify that 12 bits suffice to cover this range.

- ADC sampling rate: 2.0 MHz. A 2.0 MHz effective ADC sampling rate fits well with the time scale of the TPC signal, including the shaping time.

- Readout duration: $4.8 \mathrm{~ms}$ (in accelerator mode). The readout electronics must process and temporarily store the charge information collected during the $1.6 \mathrm{~ms}$ electron drift time. Adding the $1.6 \mathrm{~ms}$ preceding a trigger and the $3.2 \mathrm{~ms}$ following it, for a total of $4.8 \mathrm{~ms}$, allows for a full characterization of background tracks that may overlap a neutrino interaction during a triggered event drift time.

\subsubsection{Cold Electronics}

To reduce electronic noise the interconnection length between detector wire and preamplifier should be as short as possible, thus minimizing the total capacitance seen at the preamplifier input. To accomplish this, the analog front end ASICs, which includes the preamplifiers, shapers and signal drivers, reside inside the LAr cryostat, along with the wire bias voltage distribution system, decoupling capacitors, and calibration networks. 


\subsubsection{CMOS Analog Front End ASIC}

A CMOS implementation of the cold electronics was initially rejected due to concerns about long development time. Instead, the initial baseline design used JFET preamplifiers located in the cold (120K) argon gas above the liquid level. However, rapid CMOS technology development supported by the LBNE LArTPC program since the initial baseline choice enabled a second look at CMOS.

The CMOS ASIC technology offers several advantages. The nominal noise performance of the CMOS designs compares favorably to the JFET designs for the majority of wires. For wires terminating on the chamber sides, the CMOS ASIC design avoids the additional capacitance of cables to reach preamps in the gas. The CMOS ASIC design allows for an adjustable gain and filter time constant that can be better optimized for the given operating conditions. It employs fewer connectors, and its soldered connection to its motherboard offers higher reliability than JFET preamplifier hybrid pins inserted into sockets. The CMOS design allows signal shaping functions to be moved to the ASIC; and the calibration system simplifies through channel selection capability on the ASIC. Finally a robust CMOS ASIC market exists, in contrast to the limited number of potential suppliers available for supply of presorted JFET units.

Risks associated with the CMOS ASIC design include vulnerability to electrostatic discharge (ESD), concerns about operating at the $87 \mathrm{~K}$ liquid argon temperature, and possible aging issues. These risks can be mitigated through careful motherboard design that provides sufficient protection against ESD during installation, extensive tests of the ASIC and motherboard at each prototyping stage to ensure functionality at $87 \mathrm{~K}$, and performance of accelerated aging tests to verify that, though MicroBooNE run time will not test CMOS lifetime limits.

Management analyses revealed the MicroBooNE construction schedule to be compatible with CMSO development work, that migration to the CMOS technology would not affect other subsystems, that the migration would have no impact on data handling electronics and the DAQ system, and that no extra costs would be incurred. An internal review held in November 2010 validated these conclusions and resulted in a recommendation (MicroBooNE DocDB 1151) that MicroBooNE switch to the CMOS design. Following this internal review, the MicroBooNE collaboration officially adopted the CMOS front-end electronics at the December 2010 collaboration meeting.

The resulting CMOS analog front end ASIC integrates both preamplifier and shaper on a single chip. Each chip has 16 independent channels accommodating signals from 16 wires, and each channel has a charge injection capacitor for precision calibration. The shaper has four programmable gain settings $(4.7,7.8,14$ and $25 \mathrm{mV} / \mathrm{fC})$ and four programmable peaking time settings $(0.5,1.0,2.0$ and $3.0 \mu \mathrm{s})$ that provide more flexibility to the readout system. The CMOS 
ASIC's better noise performance at liquid argon temperatures allows all of the cold electronics to be submerged in the LAr, simplifying the TPC design. Induction plane wires that terminate at the side of the TPC can be treated the same as all the other wires. The CMOS ASICs consume only $\sim 10 \mathrm{~mW} /$ channel of power, compared to the JFET preamplifier's $\sim 140 \mathrm{~mW} /$ channel, effectively reducing by $\sim 1 \mathrm{~kW}$ the heat load to be handled by the cryogenics system compared to the original design. Cold electronics can be positioned independently of the cryogenic state in the MicroBooNE vessel, and the temperature distribution within the argon gas does not affect electronics operations. The TPC readout board design also simplifies. Moving the shaper circuit to the ASIC reduces the analog portion of the TPC readout board to a receiver and an ADC.

Further details of the ASIC design and performance can be found in an IEEE paper available as MicroBooNE DocDB 1153.

Figure 10.2 shows a picture of the first prototype of CMOS analog front end ASIC on a test board. The equivalent noise charge (ENC) test results shown in Figure 10.3 indicate that ENC $<700 \mathrm{e}^{-}$with $220 \mathrm{pF}$ input capacitance and $1 \mu$ s peaking time at liquid nitrogen temperature. Figure 10.4 and Figure 10.5 show the measured pulse response, along with some details on the adjustment of the gain, peaking time, and baseline. These results agree closely with simulations and indicate that the analog and the digital circuits (including the digital interface) operate as expected in the cryogenic environment. Minor revisions in the second prototype, including optimized input line parasitic resistance, should result in further improvement of ENC. Design guidelines that constrain the electric field and the current density to address the lifetime issue of CMOS devices operated at cryogenic temperatures have been applied to every single transistor in the ASIC design.

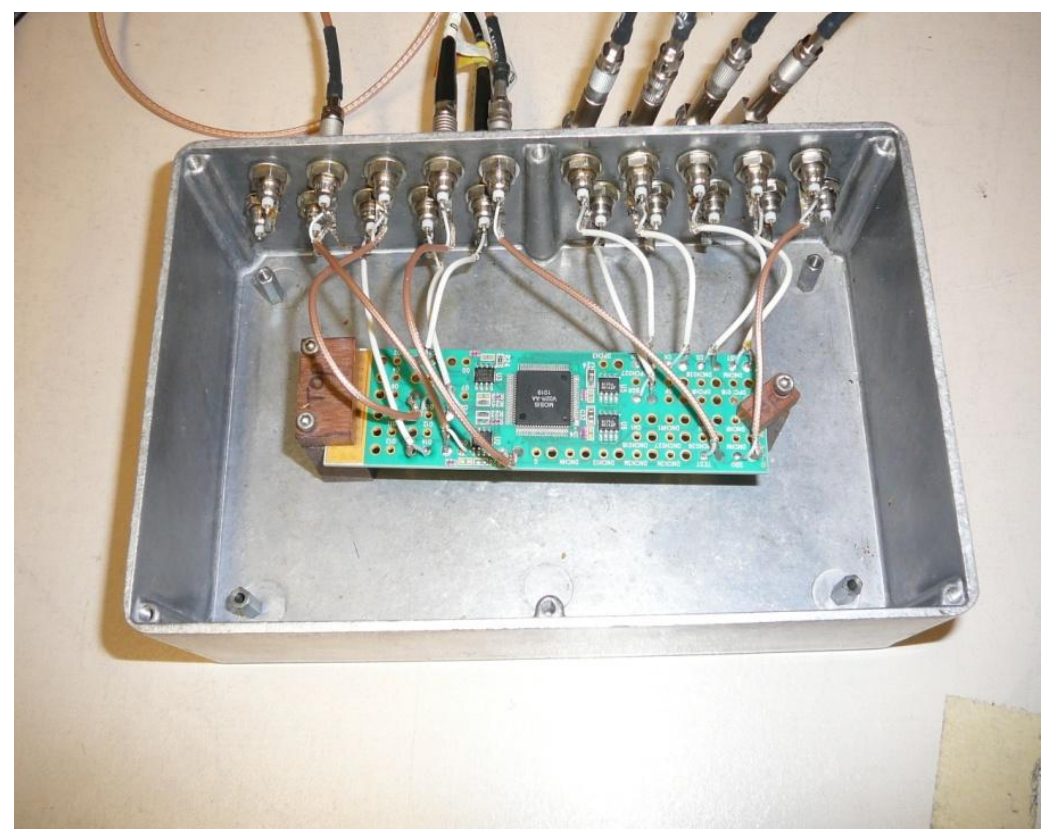

Figure 10.2 First prototype of CMOS analog front end ASIC on test board. 


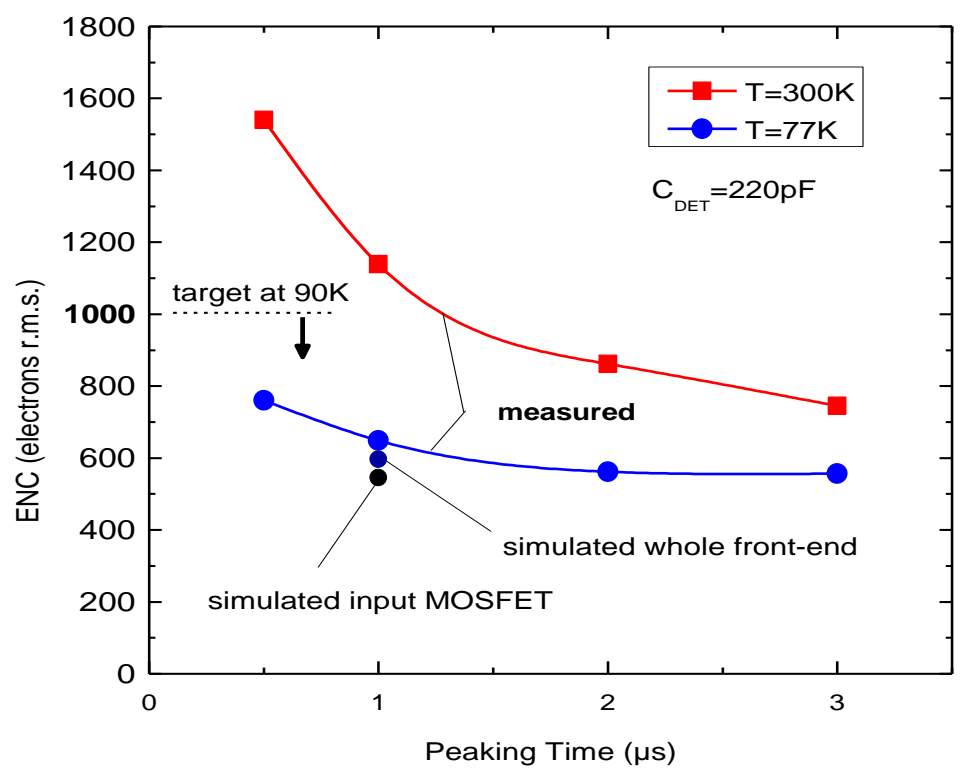

Figure 10.3 ENC measurement of CMOS ASIC.

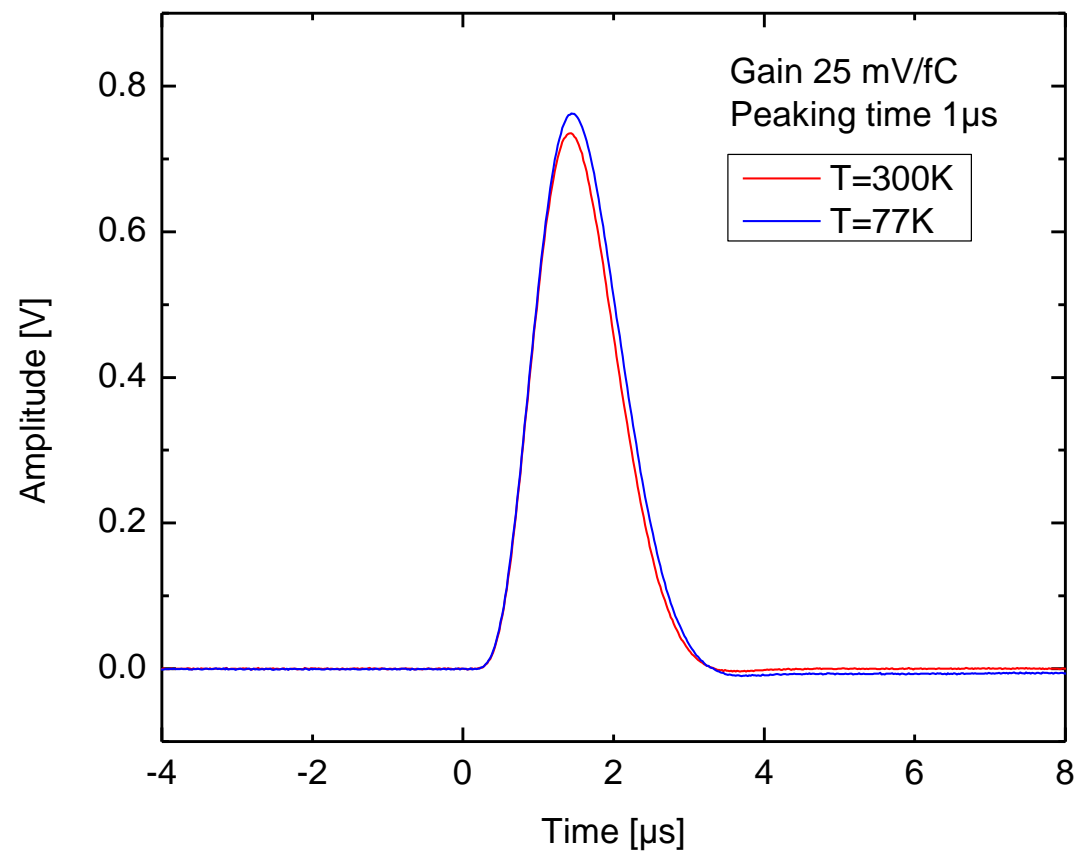

Figure 10.4 Measured pulse response of CMOS ASIC at both room and cryogenic temperatures. 


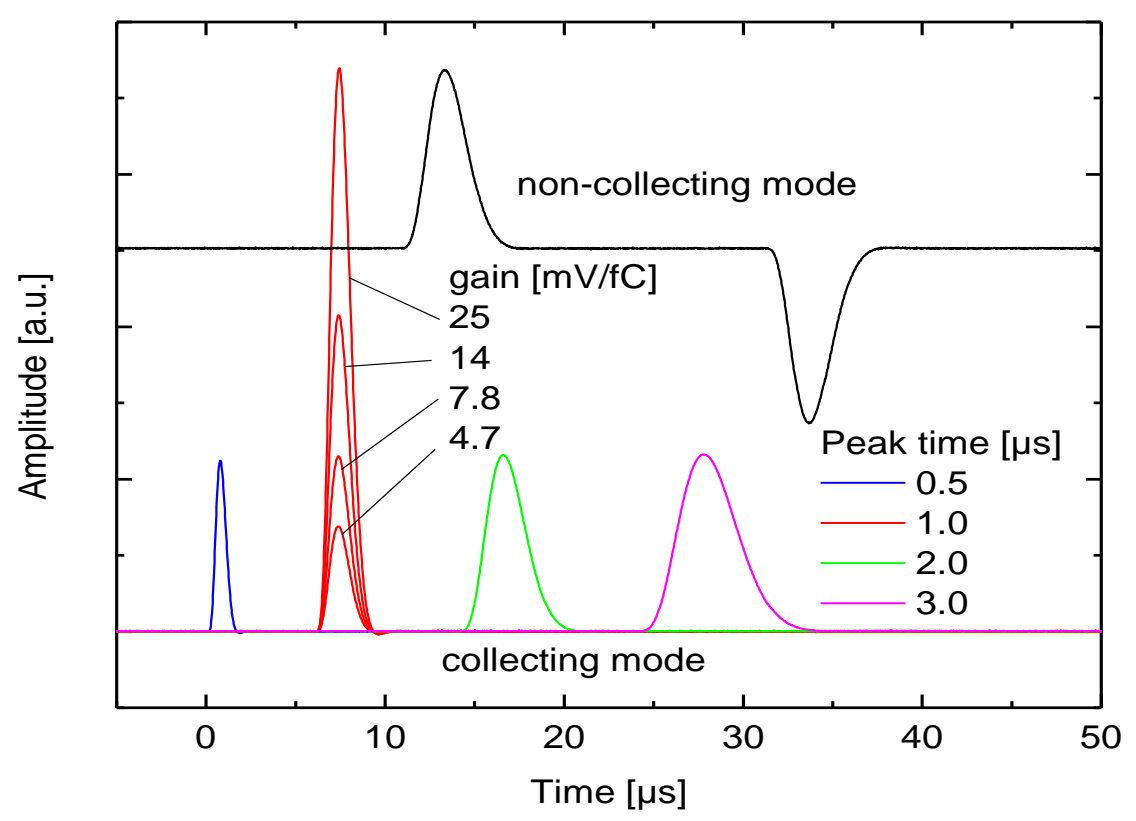

Figure 10.5 Signal measurement of CMOS ASIC with the adjustment of gain, peaking time and baseline.

\subsubsection{Cold Motherboard}

The cryogenic analog front end ASIC mounts onto a cold motherboard that provides signal interconnections between detector wires and preamplifier inputs, and between driver outputs and the cables to the signal feed-through. The cold motherboard also provides a calibration network and bias voltage distribution for wire planes. A calibration signal enters the cryostat through a feedthrough and reaches the preamplifiers through the motherboard. The calibration injection can be enabled for each preamplifier individually with a switch built into the ASIC. Each wire of the TPC receives a bias voltage between $\pm 500 \mathrm{~V}$. The bias voltage reaches the TPC wires via a twofold redundant path that allows a detector to operate normally even if one bias voltage channel fails.

Use of Rogers RO4000 series as the base material of the cold motherboard avoids potential contamination risks of LAr associated with the commonly used FR-4. The Roger RO4000 series material has a similar temperature coefficient to surface mounted components, which enhances the reliability of the electronics assembly operated at cryogenic temperatures.

The different positions of the wire attachments along the top and sides of the TPC require two types of cold motherboard. The top version of the motherboard has 192 readout channels that include $96 \mathrm{Y}$ channels, $48 \mathrm{U}$ channels, and $48 \mathrm{~V}$ channels. The side version of the motherboard has 96 readout channels that are either $\mathrm{U}$ channels or $\mathrm{V}$ channels. Figure 10.6 shows a picture of a prototype top version of a cold motherboard that has four ASIC chips (a total of 64 channels) populated . 


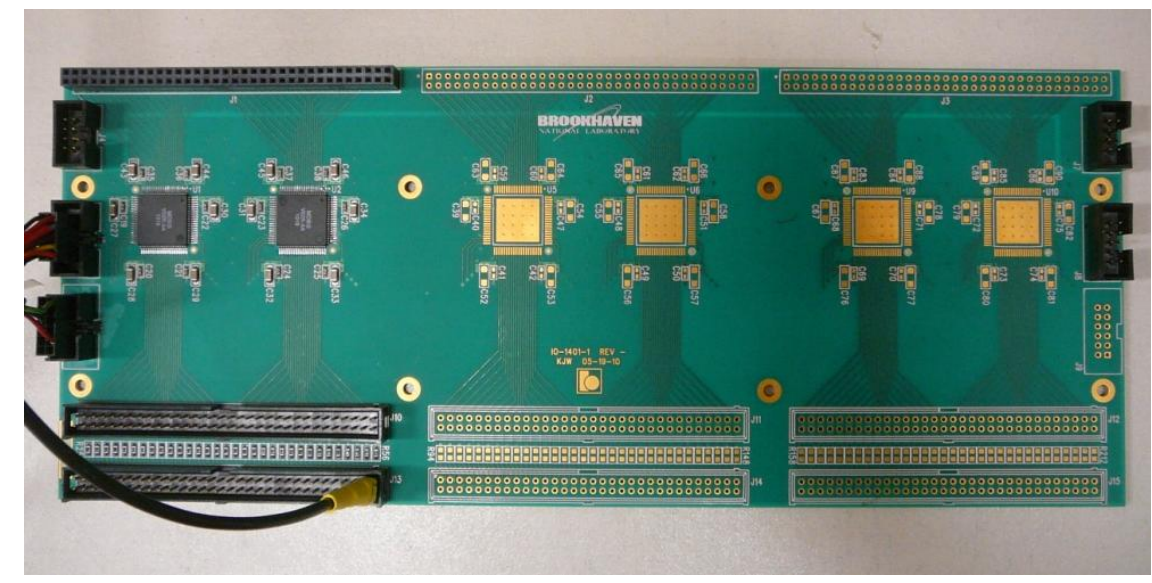

Figure 10.6 Prototype top version of cold motherboard with four ASIC chips.

\subsubsection{Warm Interface Electronics}

Each intermediate amplifier and service board mounts inside a Faraday cage on top of a signal feedthrough.

\subsubsection{Intermediate Amplifier}

The intermediate amplifiers provide $\sim 12 \mathrm{~dB}$ gain to detector signals to make them suitable for transmission to the readout electronics board in the readout crate on the electronics platform about 20 meters away. Each intermediate amplifier has 32 channels installed on the signal feed-through flange housed in a Faraday cage to provide noise isolation. Figure 10.7 shows a picture of the prototype intermediate amplifier plugged on the signal feed-through pin carrier. The intermediate amplifier uses a 68-pin SCSI-3 connector to drive the 32 channels of signal differentially for better noise immunity. The layout and connector position have been carefully designed to ensure the card can be plugged on the pin carrier in either direction. This efficiently utilizes the limited available space on the top of the feedthrough, which also makes the design of the Faraday cage easier. 


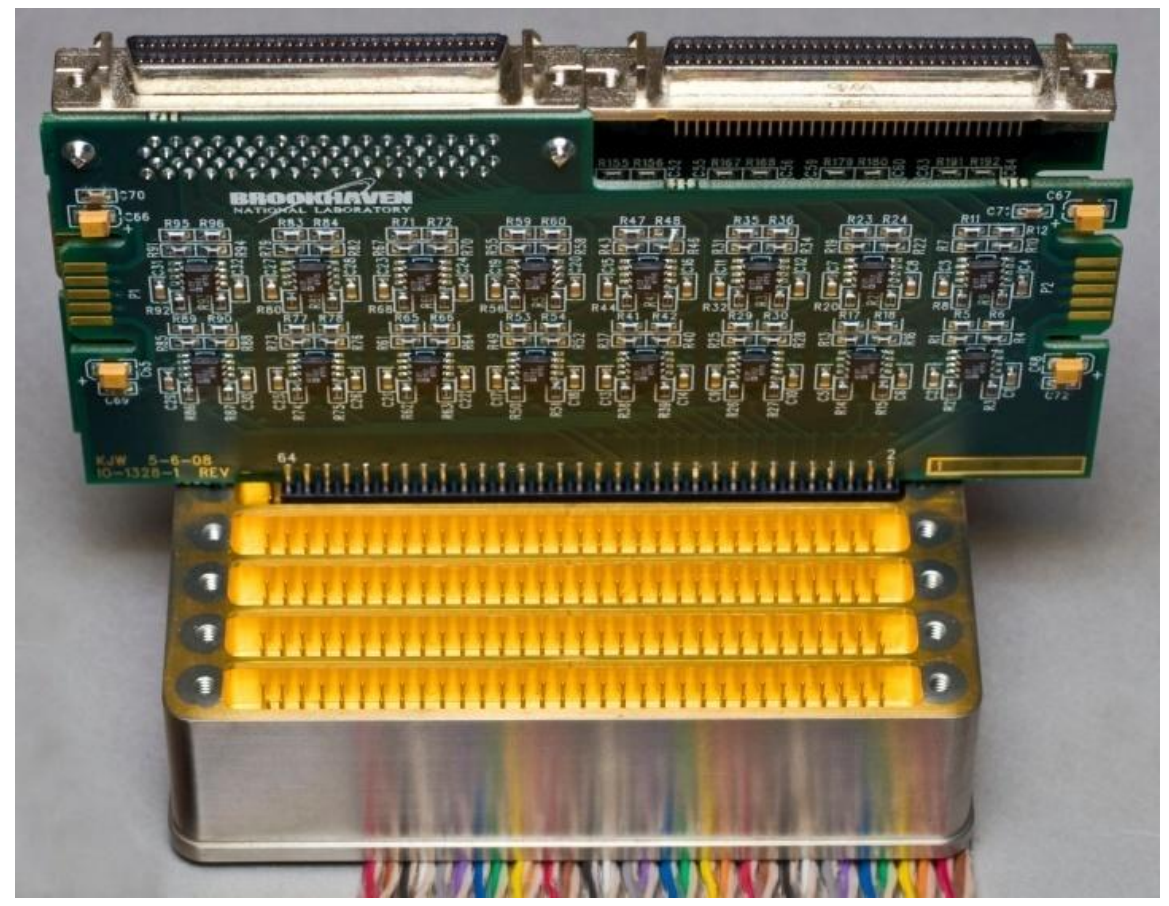

Figure 10.7 Prototype intermediate amplifier plugged on signal feedthrough pin carrier.

\subsubsection{Service Board}

The service board mounts on the top of the signal feedthrough. It provides regulated low voltage, control and monitoring signals to the analog front end ASICs. It also provides pulse injection to preamplifiers for precision calibration. Each signal feedthrough has two service boards that are responsible for control, monitoring and calibration of all readout channels on this feedthrough. The control, monitoring, and calibration signals are provided to front end electronics with twofold redundancy. Should one set of signals become defective the detector can still operate normally with the redundant set. Each service board plugs onto a 64-pin carrier row. Figure 10.8 shows a picture of the prototype service board. 


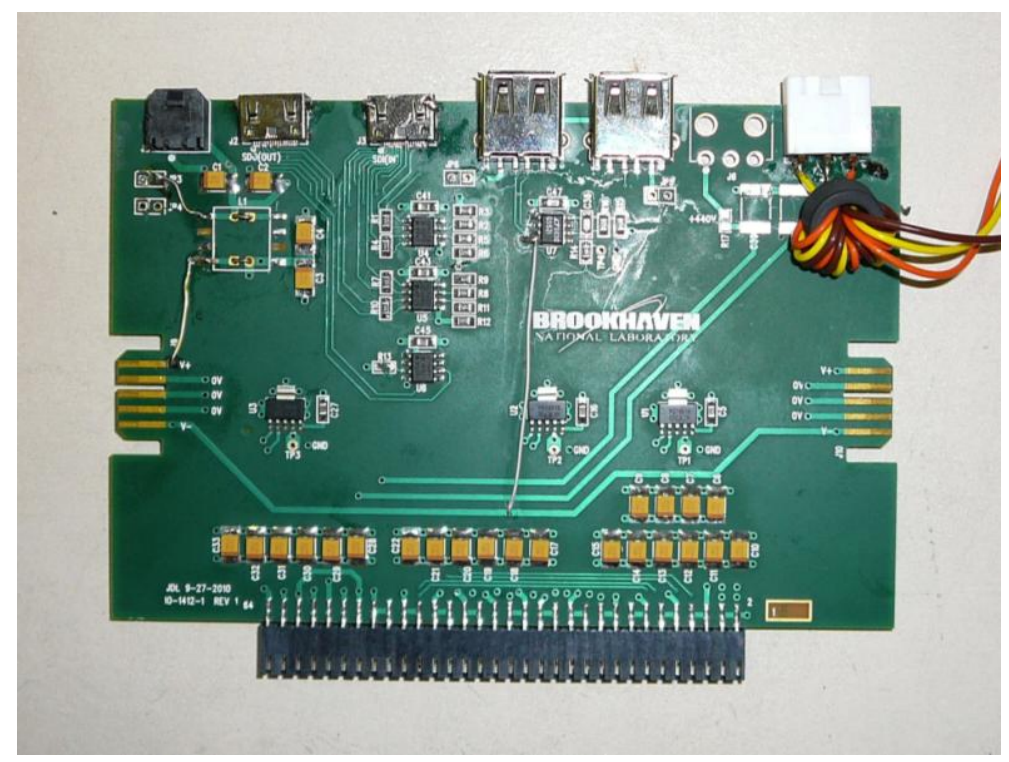

Figure 10.8 Prototype service board.

\subsubsection{ASIC Configuration Board}

The ASIC configuration board sits in the TPC front end power supply rack. It provides ASIC configuration signals drivers from a commercial off-the-shelf digital I/O board. The ASIC configuration board drives the digital serial configuration stream to the service board through a differential HDMI cable. The stream passes to the front end ASICs from the service board through the cold cable. The ASIC configuration board provides the interface between ASICs and control PC. Figure 10.9 shows a picture of the prototype ASIC configuration board mounted with an NI USB6501 digital I/O board.

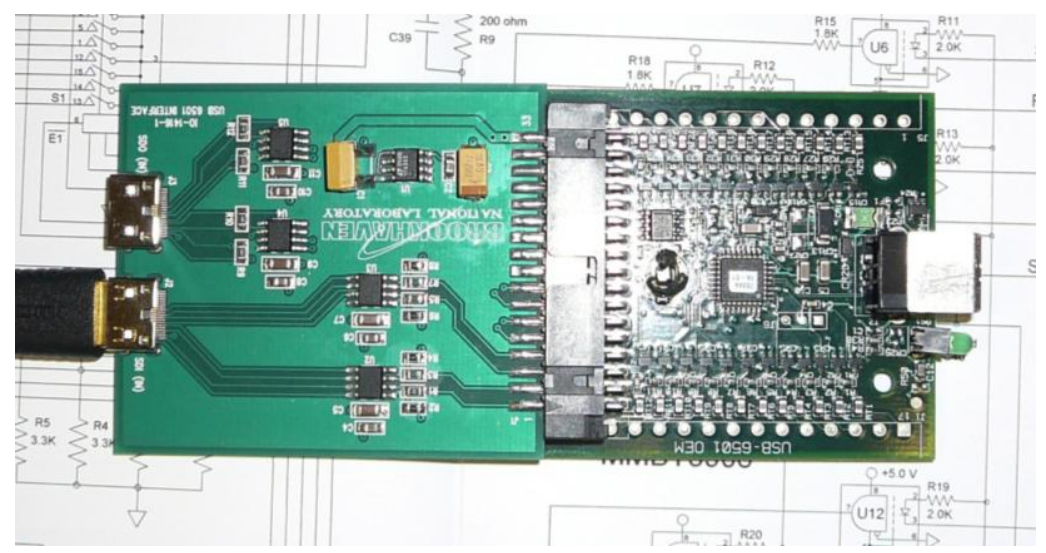

Figure 10.9 Prototype ASIC configuration board mounted with an NI USB6501 digital I/O board

\subsubsection{Digitizing and Data Handling Electronics}

The TPC readout board receives the amplified and shaped detector signals from the intermediate amplifiers; it then samples these signals continuously at a rate of $16 \mathrm{MHz}$. The digitized outputs from the ADC pass to an FPGA for data processing, data reduction, and preparation for readout 
by the DAQ system. Mechanically, sixteen readout boards reside in each of nine crates, with each crate including a custom designed backplane, a crate controller, and a transmit (XMIT) module, as shown in Figure 10.10.

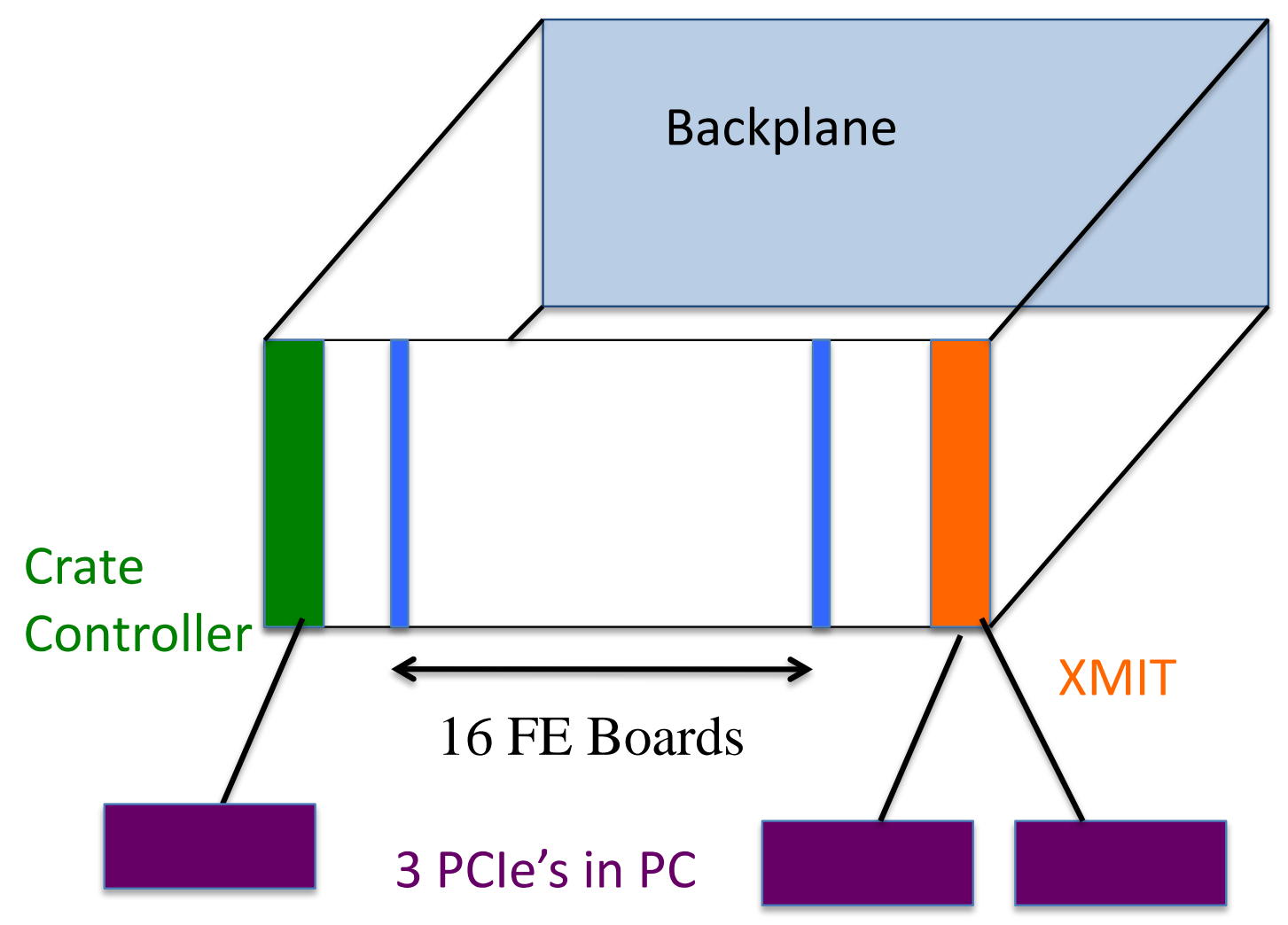

Figure 10.10 Configuration of the TPC crate.

\subsubsection{Mechanical Assembly}

The TPC readout board configures as a single readout card that reads 64 wires. It includes two parts, a digitizing section and a data handling section. Brookhaven has responsibility for the design of the digitizing section, and Columbia University's Nevis Laboratories (Nevis) has responsibility for the data handling section. The PMT readout electronics system employs an identical data handling section that employs a different analog front end. The design of the digitizing and data handling sections by different institutes requires careful coordination of the mechanical assembly, mating connector choices, signal mapping, and power supplies of this board.

Figure 10.11 shows the mechanical assembly drawing. The digitizing section forms a "C" shaped board laid out as an 8-layer printed circuit board. The data handling section consists of a 14-layer printed circuit board. The choice of a smaller board allows for short trace lengths beneficial for high speed signals. The full assembly comes together as a standard VME 9U card in height, with a $280 \mathrm{~mm}$ depth. The first mating test of the BNL receiver/ADC board with the Nevis FEM 
(front end module) board has been accomplished, and the mechanical integration has been verified successfully. Figure 10.12 shows a picture of the TPC readout board assembly.

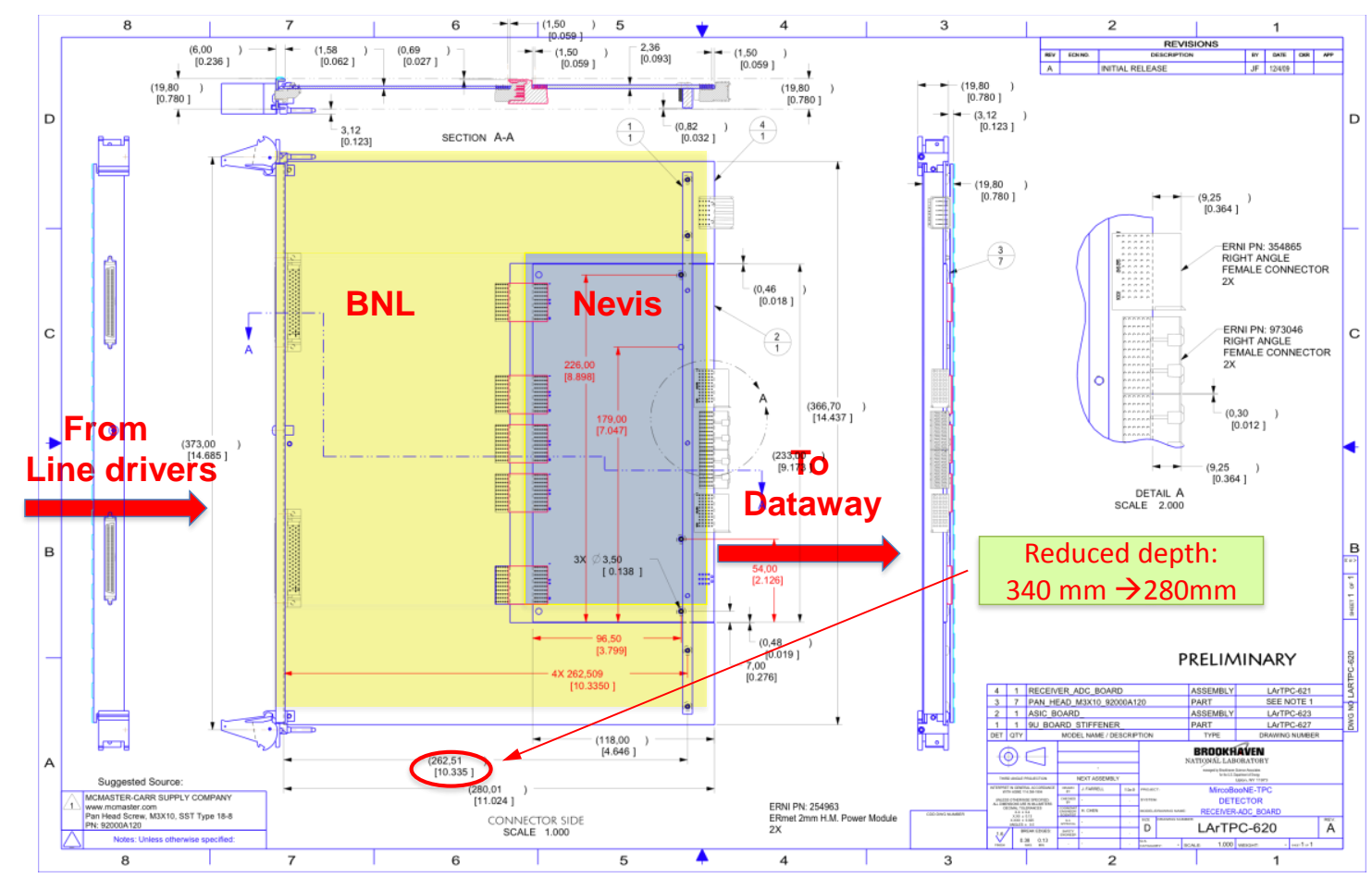

Figure 10.11 A mechanical assembly drawing of the TPC readout board showing how the BNL and Nevis boards are joined together. 


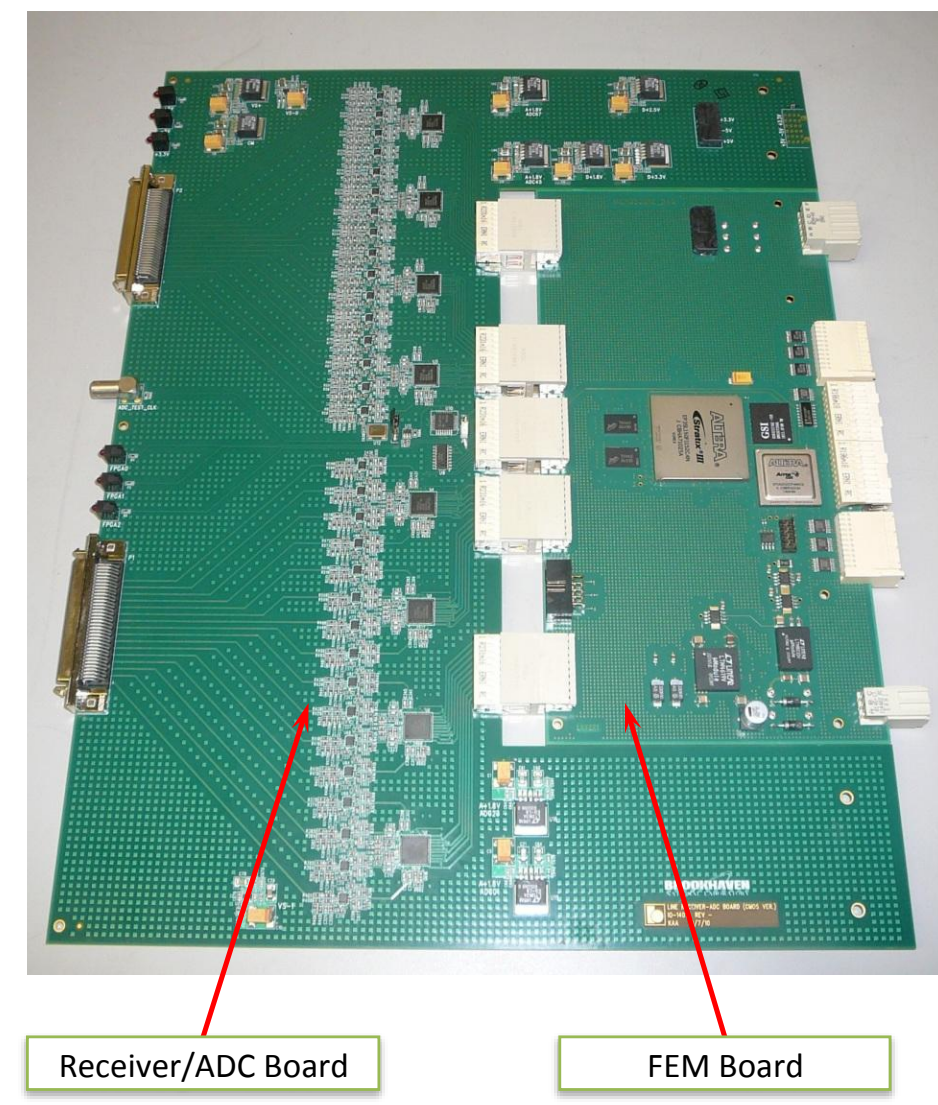

Figure 10.12 The TPC readout board prototype showing the BNL(Receiver and ADC) and Nevis(FEM) sections.

\subsubsection{Digitizing Section}

The digitizing section of the TPC readout board, also called the receiver/ADC board, includes line receivers and eight $\mathrm{ADCs}$. It receives the detector signal from the intermediate amplifiers through a $20 \mathrm{~m}$ cable and then drives the ADC input. An AD9222 octal-channel, 12-bit ADC from Analog Devices Inc. provides adequate dynamic range, as verified by Monte Carlo simulation. With a $16 \mathrm{MHz}$ sampling rate and signal processing, improvements in the signal-tonoise ratio over electronic shaping may be obtained. The AD9222 provides high integration density and low power consumption (<100 mW/channel) for the readout board. It also generates a high-speed low voltage differential signal (LVDS) serial output stream, which simplifies the number of connections needed to the FPGA. Figure 10.13 shows the simulation schematic of the analog front end, including the preamplifier, shaper, intermediate amplifier, receiver, and ADC driver for collection plane. Adjusting the signal baseline properly for the collection plane takes into account the different signal properties on collection and induction planes and ensures that the collection plane unipolar differential signals can make use of the full ADC analog input range. Figure 10.14 shows the impulse response of the circuit for the collection plane.

A clock generator will be used to generate a low jitter $16 \mathrm{MHz}$ clock to all 10 crates via a distribution board and coaxial cables. At each crate the clock signal will enter a clock fanout 
board mounted on the backplane, from which it will be delivered to all boards in the crate as a point to point LVDS signal. In addition, for single crate testing, a local $16 \mathrm{MHz}$ crystal mounted on the crate clock fanout board can also generate a local clock. The $64 \mathrm{MHz}$ clock used by the PMT readout will be generated starting from the general $16 \mathrm{MHz}$ clock.

A prototype exists for the digitizing section of the 64-channel readout board. A test stand being set up at BNL will allow for complete characterization of the full detector signal readout chain from analog front end ASIC to PC. To accomplish this test, an interface board has been designed to transfer ADC data to a Xilinx FPGA evaluation board ML605 for data acquisition. Figure 10.15 shows a picture of the prototype receiver/ADC board.
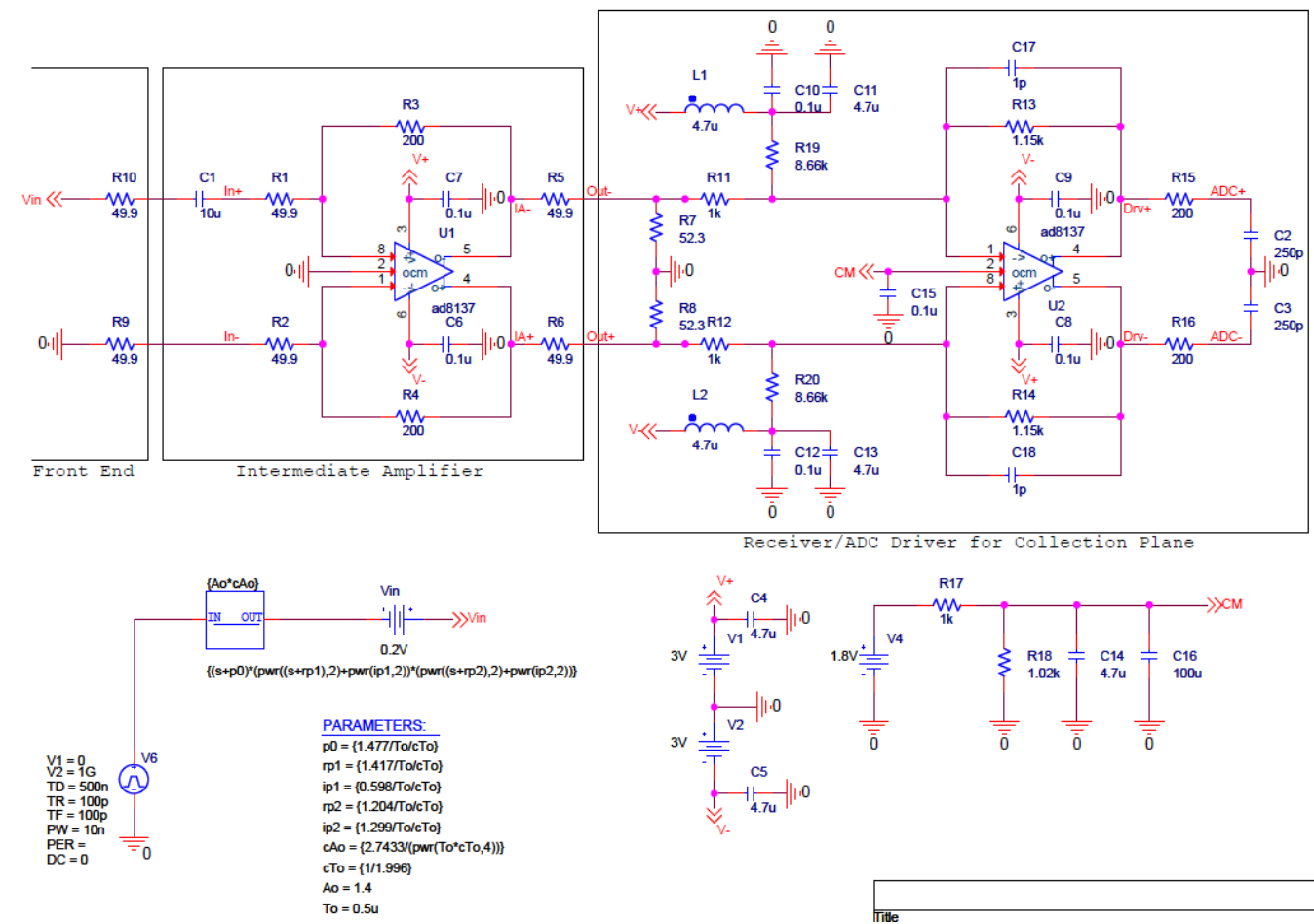

To $=0.50$

Figure 10.13 Simulation schematics of the analog front end for collection plane. 


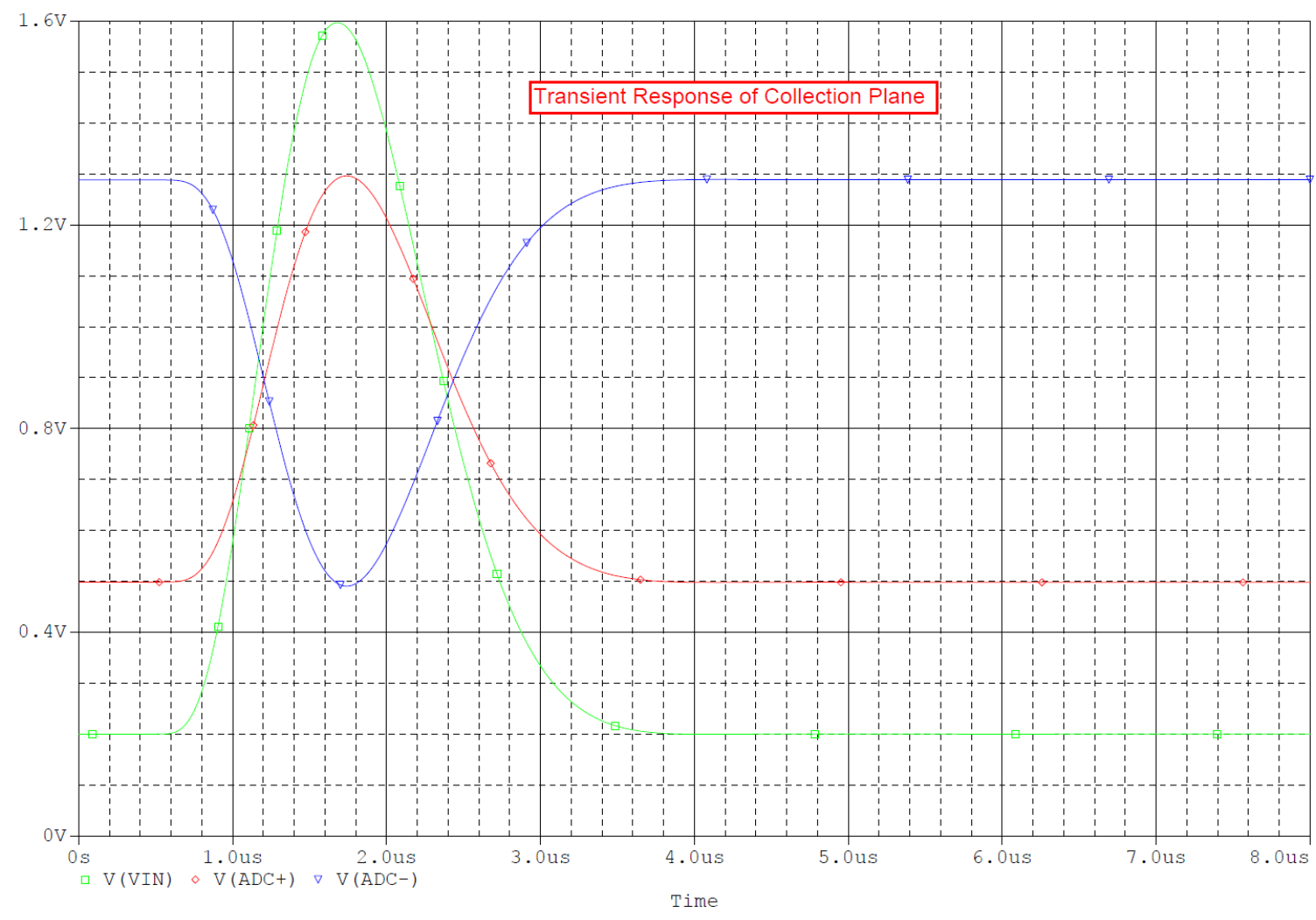

Figure 10.14 Impulse response of the analog front end for collection plane.

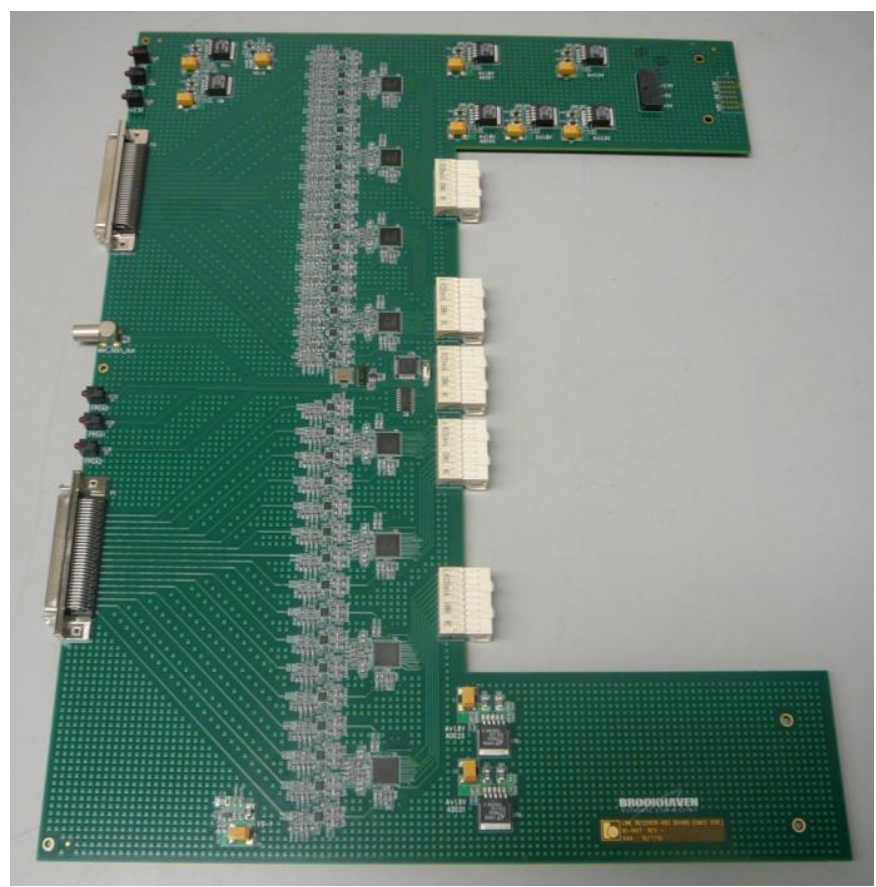

Figure 10.15 Prototype receiver/ADC board.

The MicroBooNE TDR (2/24/2012-DocDB 1821-v12): Electronics/Data Acquisition (WBS 1.5/1.9) Page 106 
Each TPC readout board processes 64 TPC wires: $32 \mathrm{Y}$ wires, $16 \mathrm{U}$ wires, and $16 \mathrm{~V}$ wires. Two SCSI-3 connectors (P1 and P2) receive the signals from the wires. Differential outputs from the ADCs connect to the FPGA through four HM-Zd connectors (J1, J2, J4 and J5) that have individual ground shielding on each differential pair. A fifth HM-Zd connector (J3) distributes clock signals, SPI signals, PMT triggers, and LED signals. Figure 10.16 depicts the signal mapping from input to output on the receiver/ADC.

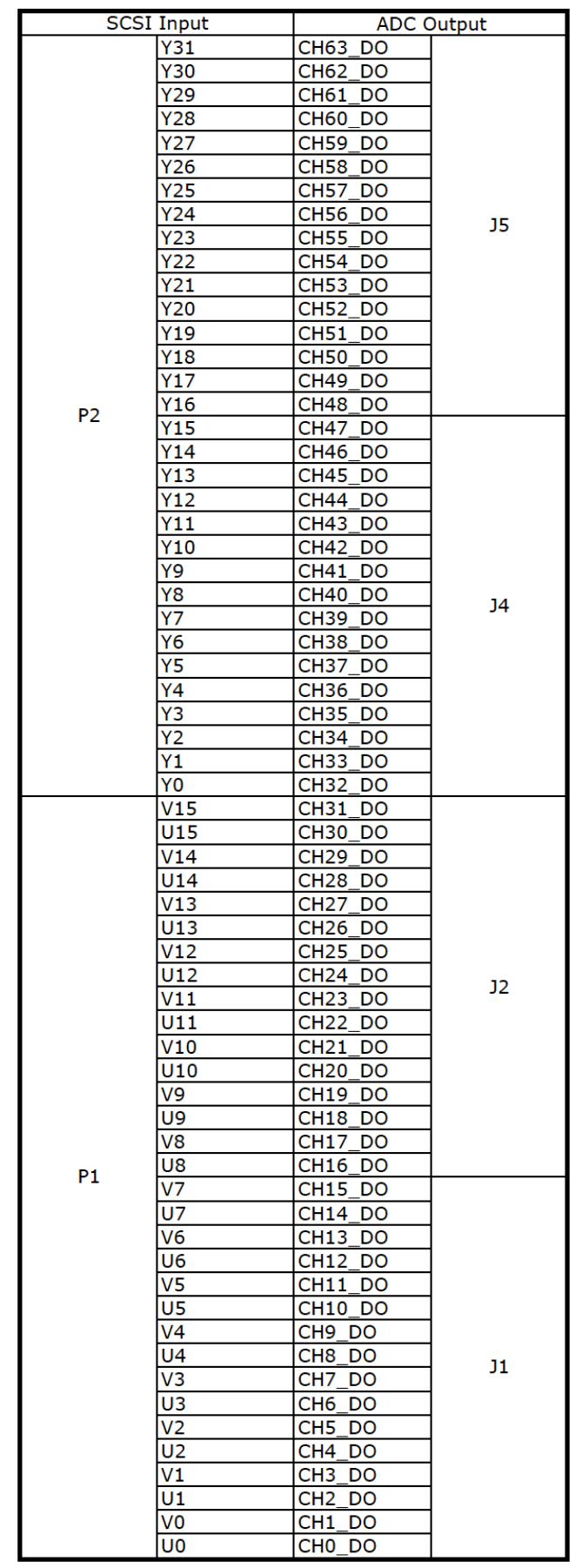

Figure 10.16 Signal mapping from input to output on receiver/ADC board. 


\subsubsection{Data Handling Section}

Figure 10.16 provides a schematic representation of the digital readout scheme. The digitized data stream moves from the ADCs to a Stratix III Altera FPGA, which reduces the sampling rate of the $\mathrm{ADC}$ to the $2 \mathrm{MHz}$ value consistent with the $1.0 \mu$ s pulse length governed by diffusion within the liquid argon drift. The FPGA stores the data from 64 wires sequentially in time in a $1 \mathrm{M} \times 36$ bit $128 \mathrm{MHz}$ SRAM memory, grouping two ADC words together in each 36 bit memory word. This requires a data storage rate of $(64 / 2) \times 2 \mathrm{MHz}=64 \mathrm{MHz}$. Since data reduction and compaction algorithms rely on the sequential time information of a given wire, the data readout out from this SRAM memory takes place in wire order in alternate clock cycles, again at the rate of $64 \mathrm{MHz}$. The SRAM chip size and memory access speed permit continuous readout of the TPC data. Since the readout clock is not synchronous with the accelerator spill time, the $4.8 \mathrm{~ms}$ worth of data relevant to an accelerator neutrino event spans four $1.6 \mathrm{~ms}$ long frames. In order to reduce the amount of data being transmitted, the FPGA trims the four frames to span the exact $4.8 \mathrm{~ms}$ required, $1.6 \mathrm{~ms}$ before the trigger plus $3.2 \mathrm{~ms}$ after the trigger.

\section{Deadtimeless FEM}

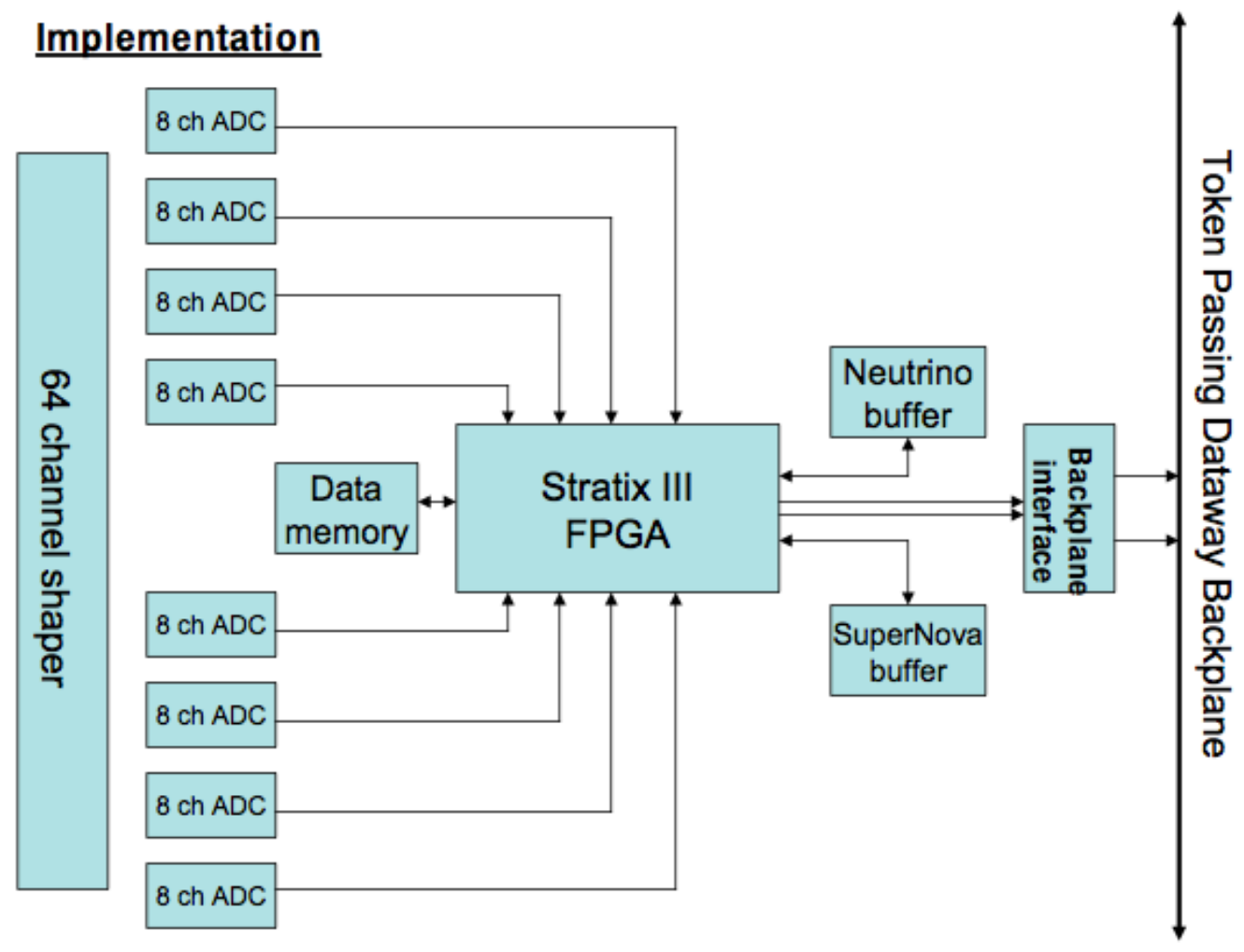

Figure 10.17 The digital readout scheme showing the 8 ADC chips, the FPGA and the memory chips.

The data divert into the beam neutrino and supernova streams depending on whether or not a trigger has occurred signaling an accelerator neutrino as shown in Figure 10.18. Different 
compaction and data reduction algorithms apply to the two streams. A lossless Huffman coding scheme implemented in the FPGA compacts the accelerator neutrino events. In addition, given the expected neutrino beam event rate of one in 10 minutes, a PMT trigger can be used to identify accelerator spills containing a neutrino interaction, allowing others to be discarded. Data can also be acquired in a trigger-less mode to measure the PMT trigger efficiency. For the supernova events, data reduction techniques such as averaging over several contiguous time slices can be used in addition to Huffman coding to reduce the effective data sampling rate. In both modes a data frame header includes the beginning frame marker, the event type, the trigger position (if any), the channel address and the word count. Separate DRAM multi-event buffers on the FEM store the accelerator and supernova data streams.

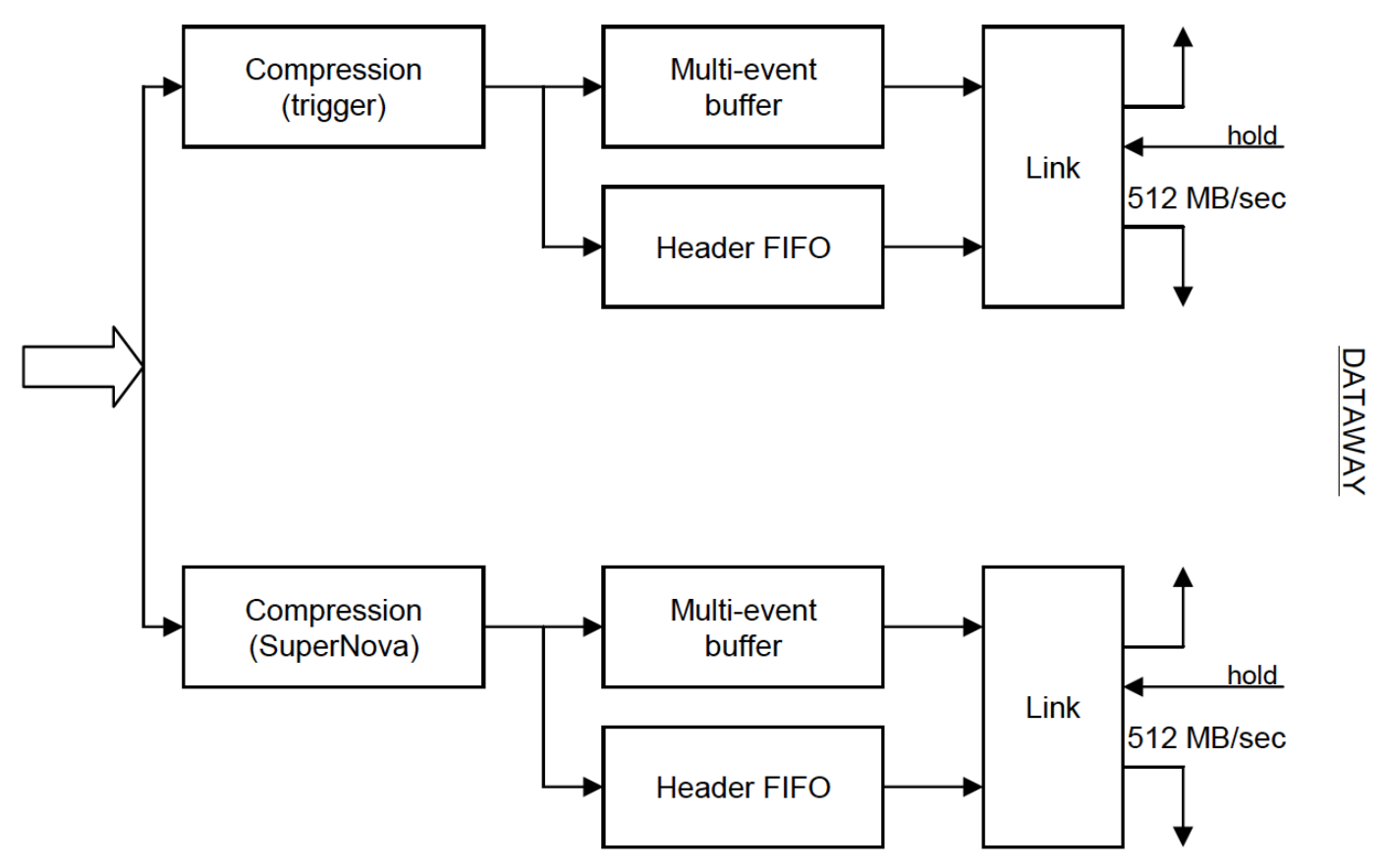

Figure 10.18 The data flow in the FEM following the split according to trigger.

Figure 10.19 displays the layout of the FEM board. The ADC data enters the board on the four connectors shown on the left of the schematic. After processing by the FPGA, the data passes to the crate backplane dataway on connectors shown on the right of the schematic. 


\section{FEM}

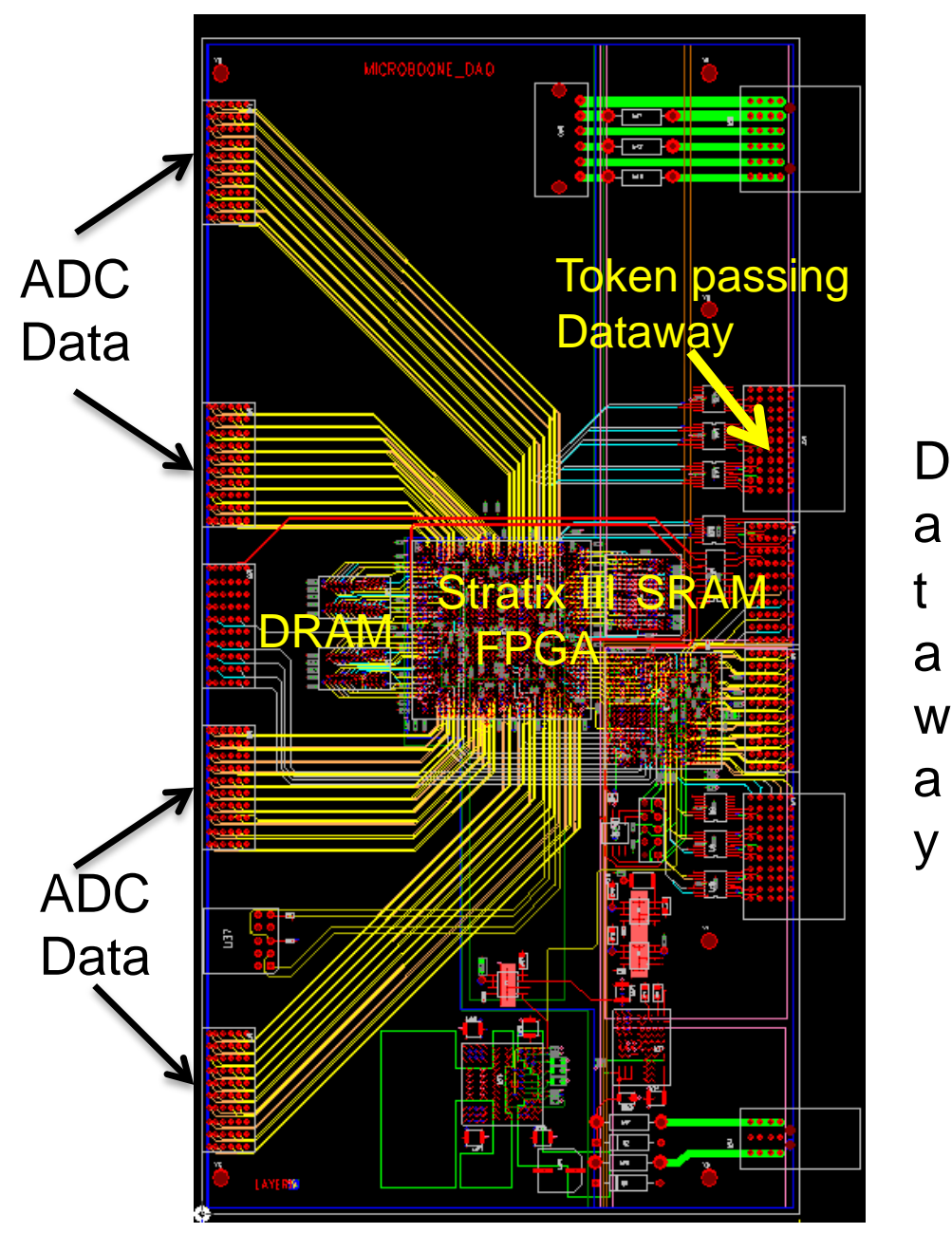

Figure 10.19 The layout of the FEM board showing the SRAM, DRAM and FPGA.

\subsubsection{Data Reduction in FPGA Stage}

The FPGA decimates the ADC data for each channel from $16 \mathrm{MHz}$ down to $2 \mathrm{MHz}$ using a standard two-step process that consists of low-pass anti-aliasing filtering and down-sampling. The low-pass filter generally takes a weighted average of multiple data samples, and therefore the decimation process increases the measurement precision by about two bits.

Experience with the Fermilab BO test stand demonstrates that on any given wire, successive data samples vary relatively slowly in time. In most cases, two adjacent data samples either coincide or differ by one ADC count. Huffman coding provides for lossless data compression by taking advantage of the slow variation of the data stream.

For accelerator neutrino events, lossless Huffman coding compression proves sufficient; but for the continuous supernova data, further compression become necessary, resulting in unavoidable 
data loss. A method called "dynamic decimation" (DD) handles this case. The DD scheme relies on recognizing regions of interest (ROI) in the data stream that contain waveforms corresponding to drift charges. Parts of the data stream not containing ROI contribute to pedestal determination. In DD the FPGA sample the ROI data the same rate as accelerator data, but reduce the pedestal sampling to a much lower rate (e.g. 1/16). The final data record of a wire contains full coverage in time, with or without drift signal from a charged track. A too-high threshold for ROI can result in loss of resolution for small signals, but the data still appear as pedestal, although sampled at lower rate. An independent Huffman coding stage further reduces the data volume after dynamic decimation.

\begin{tabular}{|l|l|}
\hline $\mathrm{U}(\mathrm{n}+1)-\mathrm{U}(\mathrm{n})$ & Code \\
\hline-4 and others & Full 16 bits word \\
\hline-3 & 000001 \\
\hline-2 & 0001 \\
\hline-1 & 01 \\
\hline 0 & 1 \\
\hline+1 & 001 \\
\hline+2 & 00001 \\
\hline+3 & 0000001 \\
\hline
\end{tabular}

\begin{tabular}{|c|c|c|c|c|c|c|c|c|c|c|c|c|c|c|}
\hline 0 & 0 & DD & \multicolumn{9}{|c|}{$\mathrm{ADC}$ value (13-bit) } & & & \\
\hline 0 & 1 & \multicolumn{10}{|c|}{$\mathrm{x}$} & & & \\
\hline 1 & 0 & 1 & 1 & 1 & 1 & 0 & 0 & & & & & & 0 & 0 \\
\hline
\end{tabular}

Figure 10.20 The data coding scheme.

Figure 10.20 shows the coding scheme for the dynamic decimation and Huffman coding. The FPGA packs the output data as 16-bit words, with the first sample represented as a raw measurement indicated by bit $(15,14)=(0,0)$, and subsequent samples represented as differences with respect to the previous samples, i.e., $\mathrm{U}(\mathrm{n}+1)-\mathrm{U}(\mathrm{n})$. A simple Huffman coding table, shown in Figure 10.20, encodes the differences with variable code lengths. The most frequent differences are assigned the code with the shortest length. The Huffman codes concatenate into the 16-bit data word with bit $(15)=1$. In the example shown in Figure 10.20, six data samples pack into the 16-bit data word. If a sample differs from its predecessor by more than \pm 3 , it assumes the value of the raw measurement word. For supernova data, bit(13) signals entering or leaving a ROI with $\operatorname{bit}(13)=\mathrm{DD}=$ either 0 or $1 . \mathrm{DD}=0$ indicates that subsequent samples lie within the ROI with normal sampling rate, while $\mathrm{DD}=1$ indicates data in the pedestal region with $(1 / 16)$ sampling rate.

Tests on BO detector data shows that dynamic decimation reduces the number of samples by about a factor of 8-12 on typical events, with a further compression ratio from Huffman coding of about 11. The Huffman coding compression drops to 6 when applied after decimation by a factor of 5. However using anti-aliasing filtering instead of straight decimation recovers most of this data reduction loss.

The blocks for dynamic decimation and Huffman coding have been test designed, compiled, and simulated at $250 \mathrm{MHz}$ in an Altera Cyclone III FPGA device. The coding for the two blocks requires roughly the same number of logic elements, about 462 of the available 39600.

Two compression strategies remain viable for neutrino beam data, as illustrated in Figure 10.21. One fully pipelines the data flow, reading the data out of the SRAM, compressing it in the 
FPGA, and storing it in the neutrino buffer. The compression will be a pipeline to match the rate of data flow. In the other strategy, the FPGA reads data out of the SRAM and temporarily stores it in a buffer as it compresses it; it then stores the compressed data back in the buffer before sending it on to the PC. This allows a compression scheme that can take as long as the time between successive accelerator spills.

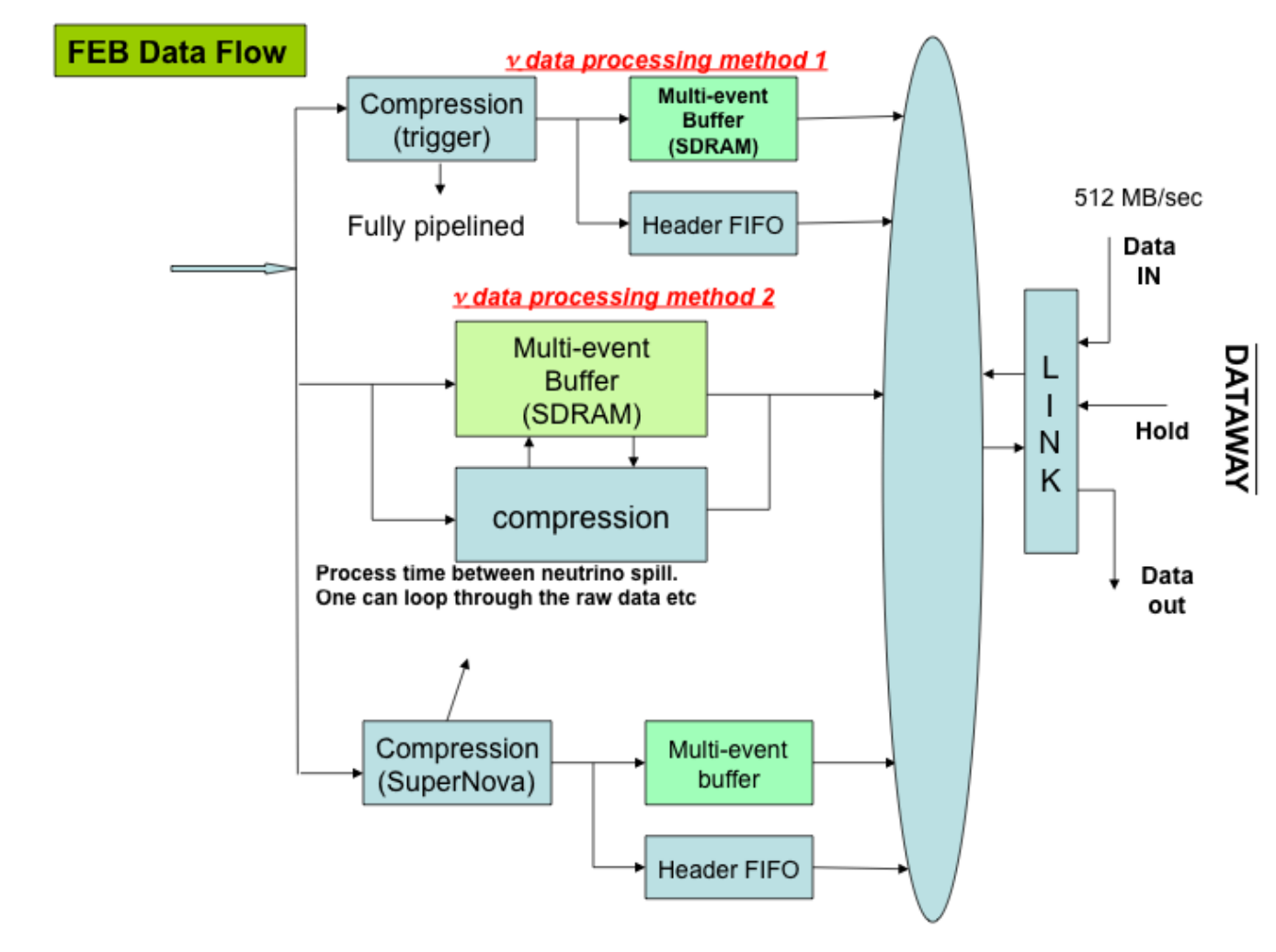

Figure 10.21 The two compression strategies described in the text.

\subsubsection{Cabling and Signal Feedthrough}

Cold cable, warm cable, and signal feedthroughs comprise the key parts for signal transmission from the TPC to the readout electronics system. Cold cable transmits detector signal from the cold motherboard to the intermediate amplifier sitting on top of the signal feedthrough, and it distributes power to analog front end ASICs. The baseline design for the cold cable consists of custom built 32-pair twisted pair flat cable with Teflon FEP insulation, AWG 26 solid core and silver plated conductor, and $100 \Omega( \pm 10 \%)$ impedance. A 64-pin 0.100 " spacing dual row crimping connector completes full cable assembly. Custom designed shells with jack screws used in the cable assembly ensure proper alignment of insertion on the signal feedthrough pin carriers. Figure 10.22 shows a picture of a prototype cold cable assembly. 
Warm cable transmits signals from the intermediate amplifier to the receiver/ADC board plugged into the readout crate. The baseline design of the warm cable consists of commercial 68-pin SCSI-3 cable. The cable assembly includes 34 differential pairs and two MicroDensity connectors. Its aluminum-foil shielding with $10 \%$ overlap functions as a Faraday cage extension. This cable offers low cost and commercial off-the-shelf availability for short cable lengths, with longer custom built cables obtainable from multiple vendors.

The signal feedthrough design must accommodate $100 \%$ hermeticity and high signal density. The ATLAS style pin carrier designed for a LAr Calorimeter satisfies these requirements. Its available pin carrier scheme implies no need for additional engineering design. Two 8-row pin carriers and two 7-row pin carriers weld onto a 14-inch CF flange with the Faraday cage mounted on top to provide the shielding for intermediate amplifiers. The bias voltage feedthrough built onto a small 2.75-inch CF flange welded onto the signal feedthrough flange routes $+440 \mathrm{~V}$ and $-204 \mathrm{~V}$ bias voltages to the detector wire planes. A filter board mounted on the bias voltage flange filters noise and ensures a good ground connection. Figure 10.23 presents a picture of the signal feedthrough assembly with electronics boards, bias voltage feedthrough, and Faraday cage. Figure 10.24 gives the pin assignment of a standard feedthrough.

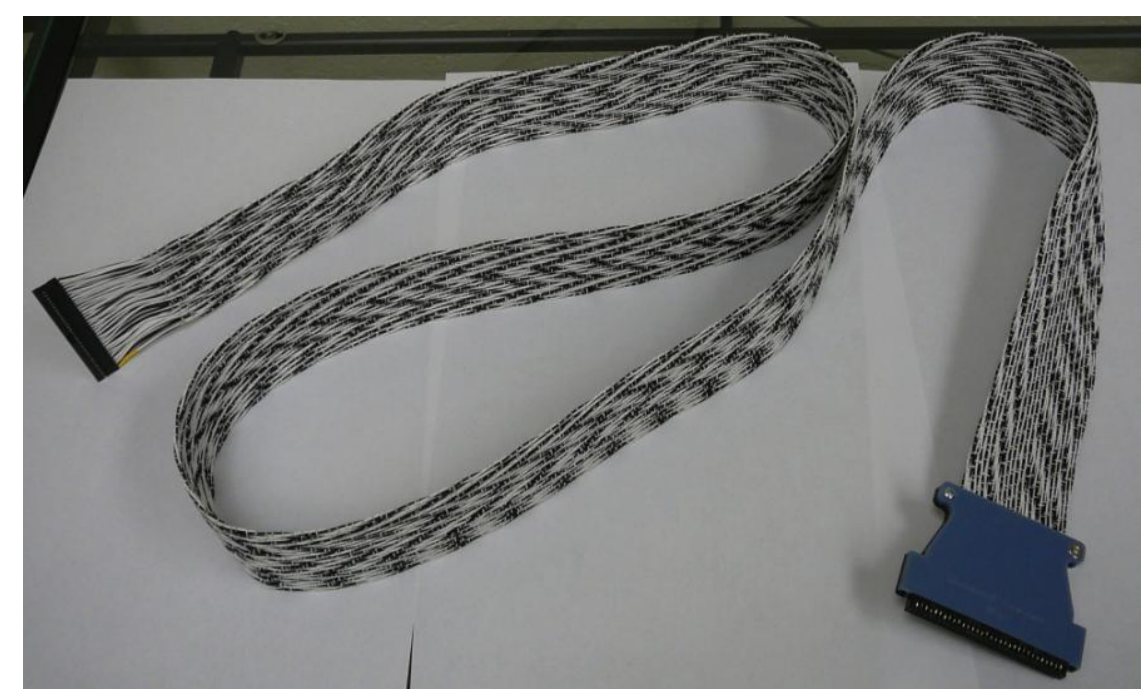

Figure 10.22 Prototype cold cable assembly. 


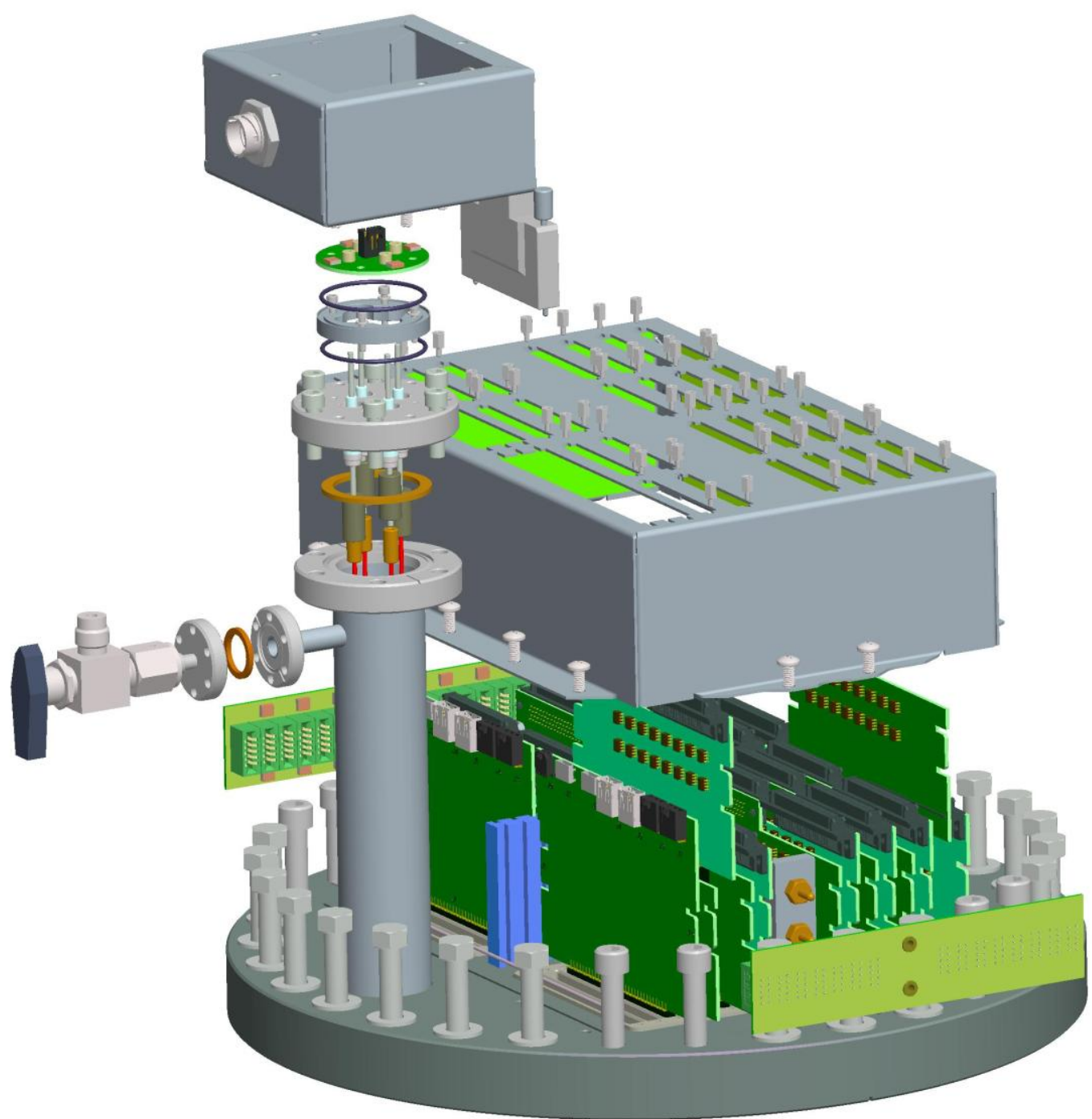

Figure 10.23 Signal feedthrough assembly with electronics boards, bias voltage feedthrough and Faraday cage. 


\title{
Pin Asssignments
}

\author{
Standard Feedthrough
}

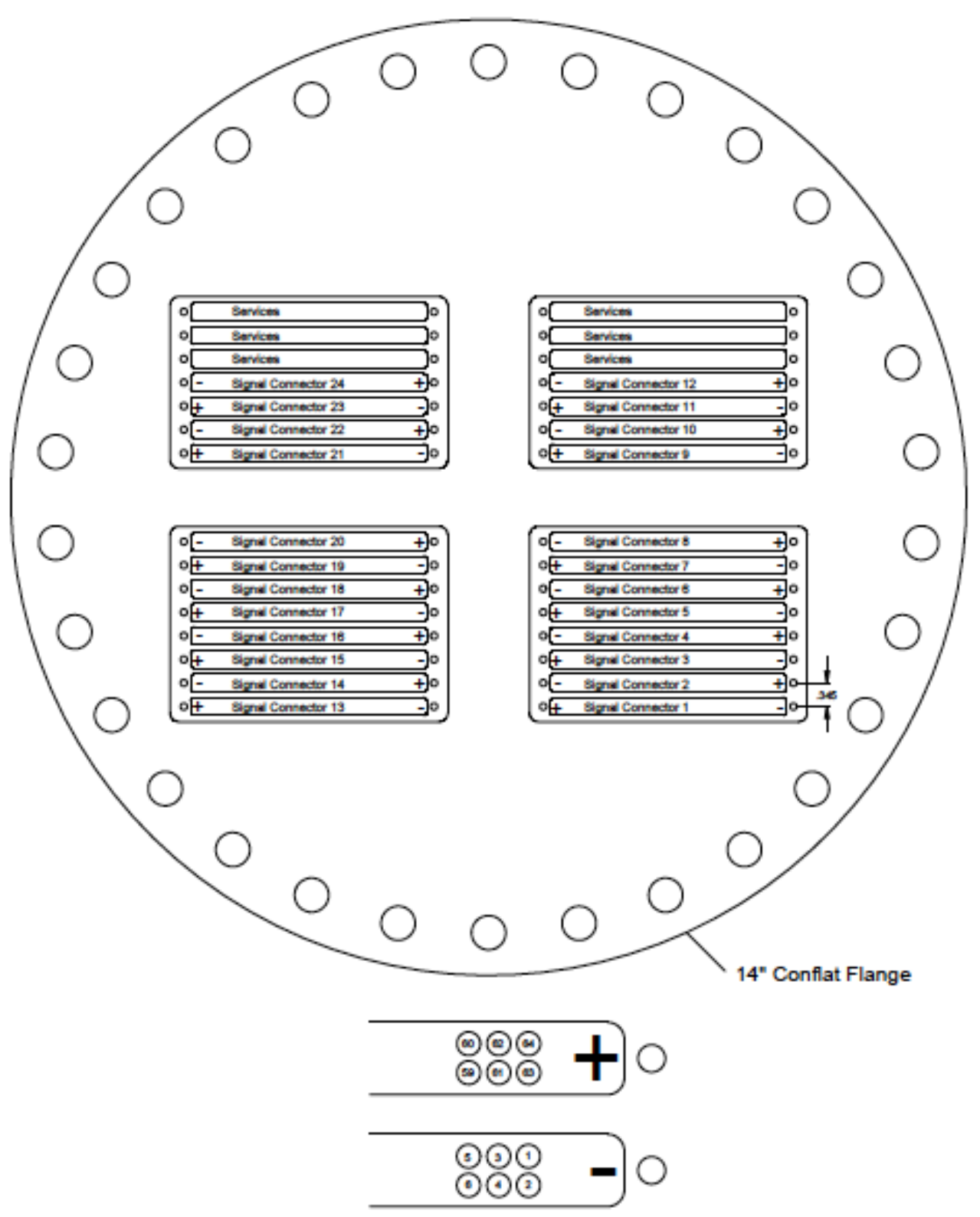

Top View looking into Feedthrough

Figure 10.24 Pin assignments of the MicroBooNE standard signal feedthrough. 
A total of 8,256 readout signal channels leave the MicroBooNE TPC. 3,456 readout channels on the $Y$ plane, and 2,400 readout signal channels on each of $U$ and $V$ planes. In the $U$ and $V$ planes, 1728 channels read out along the z-direction from the top of the TPC, and 672 channels read out along the y-direction from the sides of the TPC.

Eleven feedthroughs serve for signal readout, monitoring, calibration, and power distribution. Each feedthrough contains flanges with four pin carriers (two 8-row and two 7-row pin carriers), and each pin carrier row has 64 pins for 32 signal channels read out differentially. Nine standard feedthroughs have the same configuration to read out signals from the top of the three wire planes along the $\mathrm{Z}$ direction, and two special feedthroughs read out signals from $\mathrm{U}$ and $\mathrm{V}$ planes along the $\mathrm{Y}$ direction. Table 10.1 shows how readout signals are mapped in each feedthrough.

\begin{tabular}{|c|c|c|c|c|c|c|c|}
\hline & \multirow{2}{*}{$\begin{array}{c}\# \text { of } \\
\text { feed- }\end{array}$} & \multicolumn{2}{|c|}{ Y Plane } & \multicolumn{2}{c|}{ U Plane } & \multicolumn{2}{c|}{ V Plane } \\
\cline { 3 - 8 } & through & $\begin{array}{c}\text { 64-pin } \\
\text { row }\end{array}$ & Channels & $\begin{array}{c}\text { 64-pin } \\
\text { row }\end{array}$ & Channels & $\begin{array}{c}\text { 64-pin } \\
\text { row }\end{array}$ & Channels \\
\hline Standard feed-through & 9 & 12 & 384 & 6 & 192 & 6 & 192 \\
\hline Special feed-through A & 1 & 0 & 0 & 21 & 672 & 0 & 0 \\
\hline Special feed-through B & 1 & 0 & 0 & 0 & 0 & 21 & 672 \\
\hline Total & 11 & 108 & 3456 & 75 & 2400 & 75 & 2400 \\
\hline
\end{tabular}

Table 10.1 Readout channel mapping in the feedthroughs.

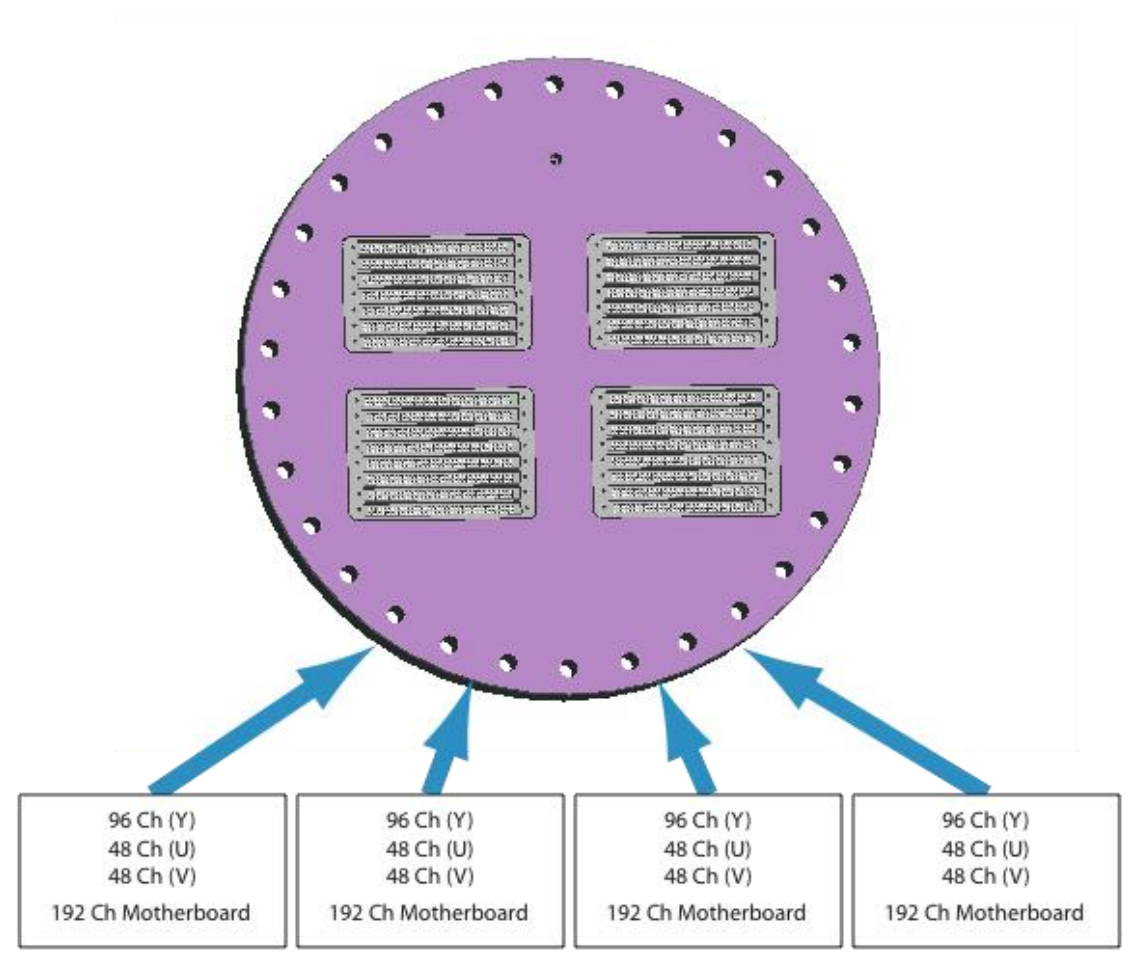

Figure 10.25 Channel mapping of the standard feedthrough. 


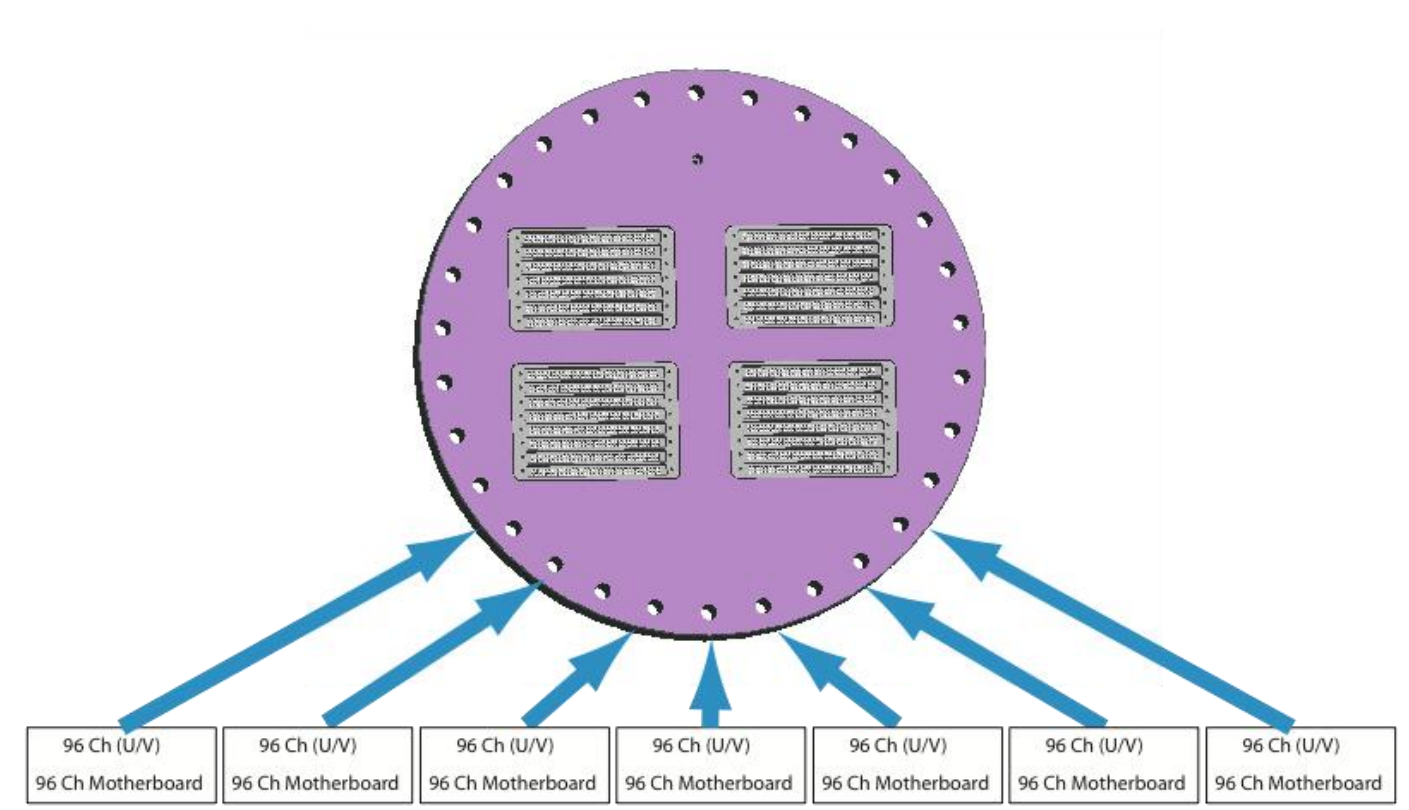

Figure 10.26 Channel mapping of the special feedthrough.

Each standard feedthrough accommodates 384 Y plane channels, 192 U plane channels, and 192 $\mathrm{V}$ plane channels, for a total count of 768 channels, reading out a $1152 \mathrm{~mm}$ slice of the TPC along the Z-direction of the wire planes. Four cold motherboards equipped with analog front end ASICs supply each standard feedthrough. Each cold motherboard houses wire connections and ASICs for 192 channels. The signal readout uses a total of 24 64-pin carrier rows per standard feedthrough. Figure 10.25 shows the channel mapping of the standard feedthrough.

The special feedthroughs each read out a total of 672 channels, which cover $2328 \mathrm{~mm}$ along the y-direction of the U or V plane. Seven cold motherboards supply each special feedthrough. Each motherboard houses wire connections and ASICs for 96 channels. The signal readout uses a total of 21 64-pin carrier rows per special feedthrough. Figure 10.26 shows the channel mapping of the special feedthrough.

\subsection{PMT Readout Electronics}

The electronics for the PMT system consists of signal shaper boards, a front end module (FEM) modified from the TPC version, a PMT feedthrough, HV/signal splitters, and a trigger board.

\subsubsection{PMT Signal Shaping and FEM}

A single cable and a special feedthrough at the cryostat provide the $\mathrm{HV}$ and signal connection to each of the 30 PMT bases in the LAr. A splitter that separates the signal from the HV routes the signal to the readout. The readout consists of a modification of the FEM used for the TPC readout, with Nevis developing both analog and digital sections of this PMT card. A modification to the analog section accommodates the shorter PMT pulses, providing appropriate shaping and 
continuous digitization at $64 \mathrm{MHz}$ (16 ns sampling) with a Texas Instrument ADS5272 ADC. Figure 10.27 shows the PMT crate layout. The shapers configure into three modules, each containing 16 channels. A single modified FEM comprising 48 channels proves sufficient to read out the whole PMT array. The PMT crate also houses the trigger board (TB).

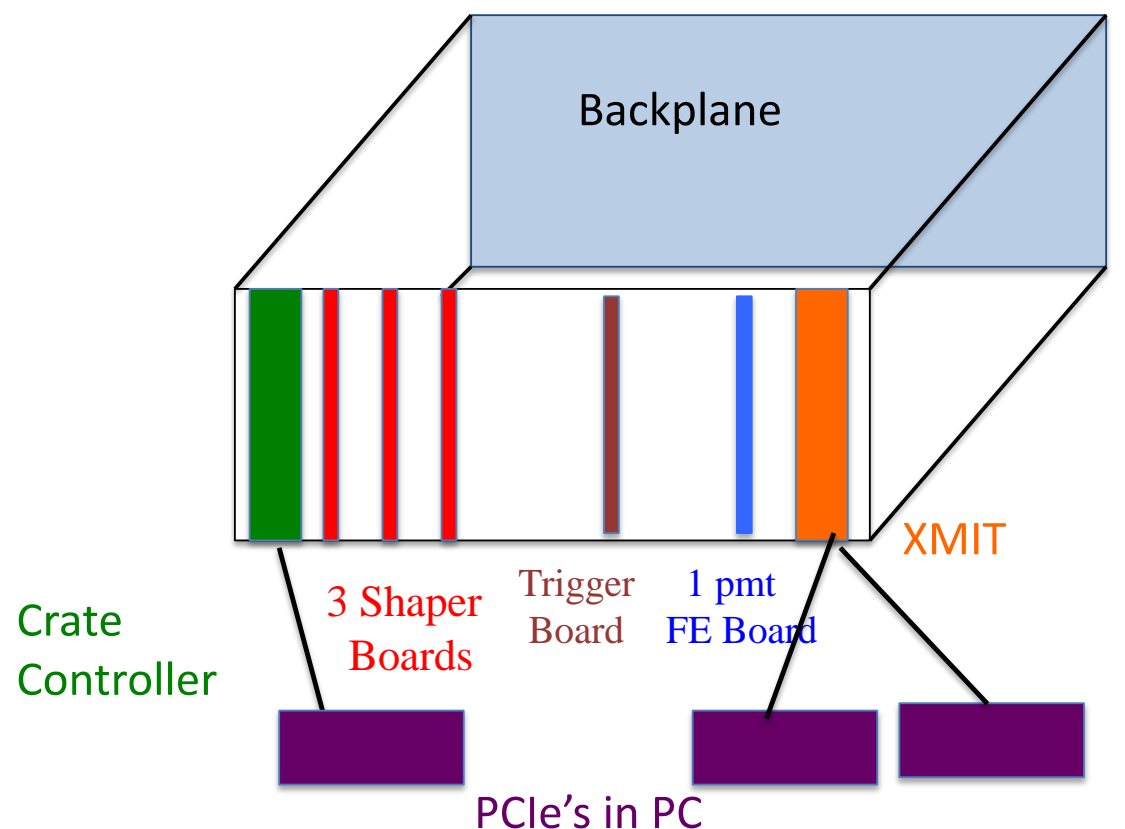

Figure 10.27 Configuration of the PMT readout crate.

\section{PMT Shaper}

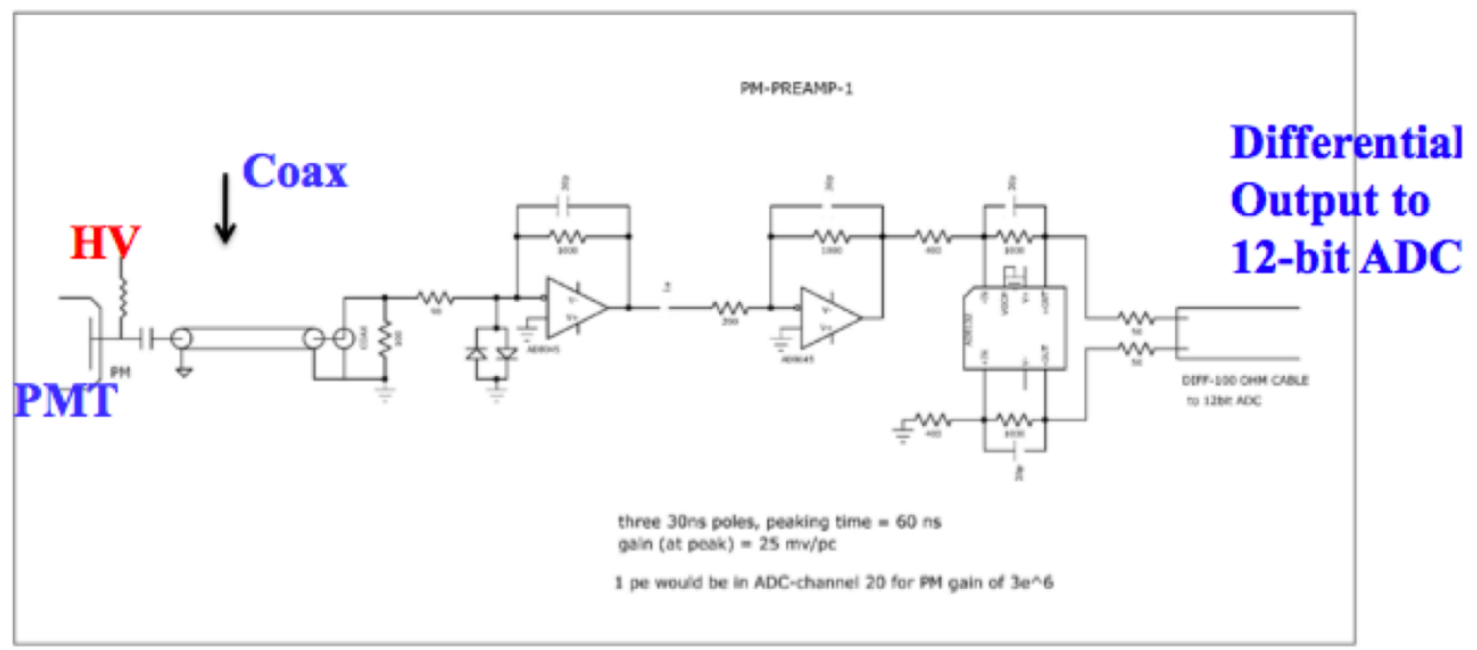

Figure 10.28 The PMT shaper schematics. 
Figure 10.28 summarizes the design of the shaper. A single channel shaper prototype tested with PMT pulses provides the output shown in Figure 10.29. The 60 ns peaking time allows digitization of two or three samples on the rising edge. This in turn enables an accurate determination of the event start time (T0) needed to determine the $\mathrm{X}$ coordinates of ionization signals in the event along the drift direction. A good time measurement also helps reject other tracks, such as cosmic rays, that cross the detector during the drift time.

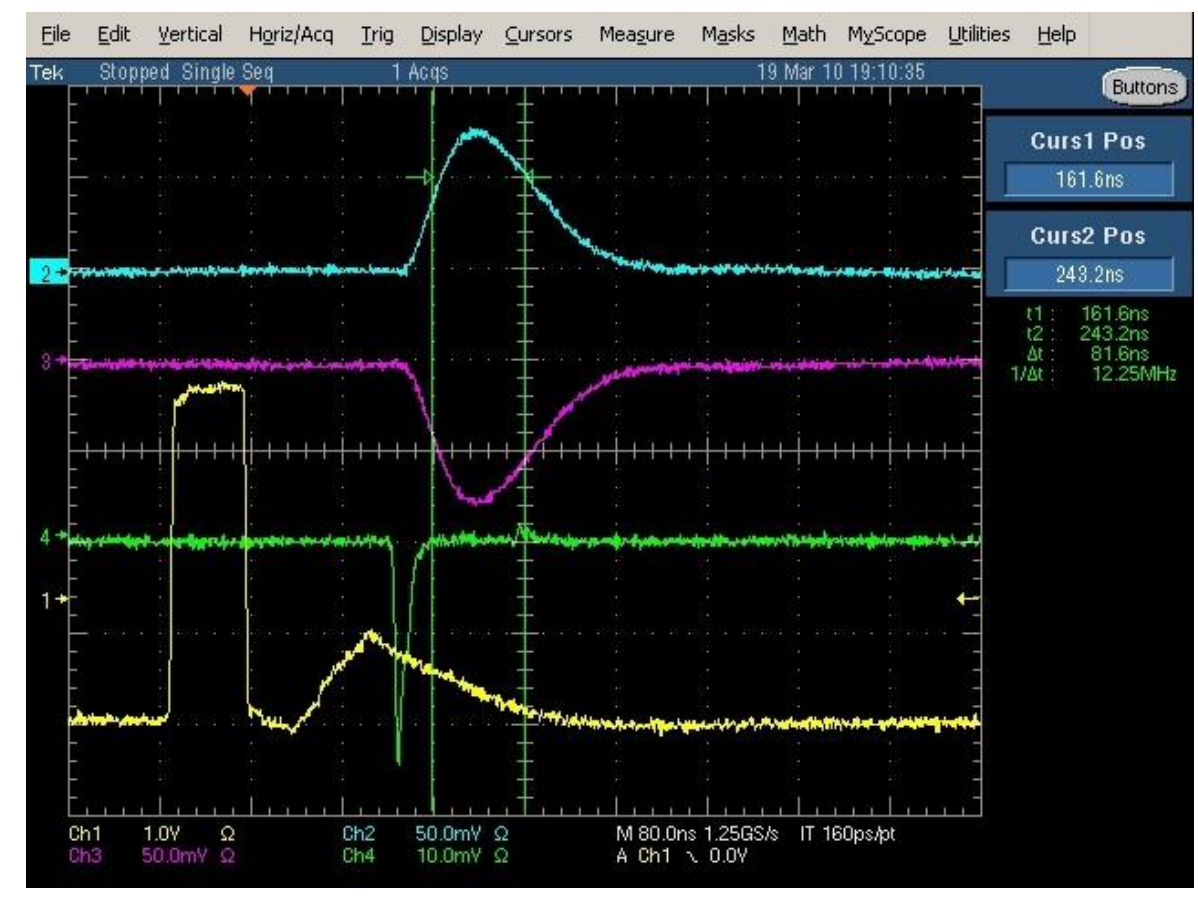

Figure 10.29 The PMT Shaper output. The bottom trace is the LED trigger pulse, the middle trace the PMT output and the top trace the shaper output.

The FEM provides the capability to apply gain corrections to individual PMT signals. Photomultiplier tube information must be recorded for the full four time frames associated with a beam neutrino event. To avoid the inordinate amount of data that would be generated at this sampling rate, the FEM applies a zero-suppression immediately after digitization, retaining samples above a given threshold, as well as enough information before and after the useful data to establish a local baseline value.

\subsubsection{Trigger Board}

The trigger board (TB), shown in Figure 10.30, flags time frames that must be treated differently than those for continuous reading of supernova events. The FPGA firmware in the PMT FEM generates one or more triggers based on the PMT signals. Example trigger conditions include: sum of all 30 PMT pulse heights above a threshold, sums of groups of PMT pulse heights above a lower threshold, number of PMT above threshold satisfying a multiplicity requirement, and PMT pulse height pattern consistent with a delayed Michel electron from stopped muon decay. 


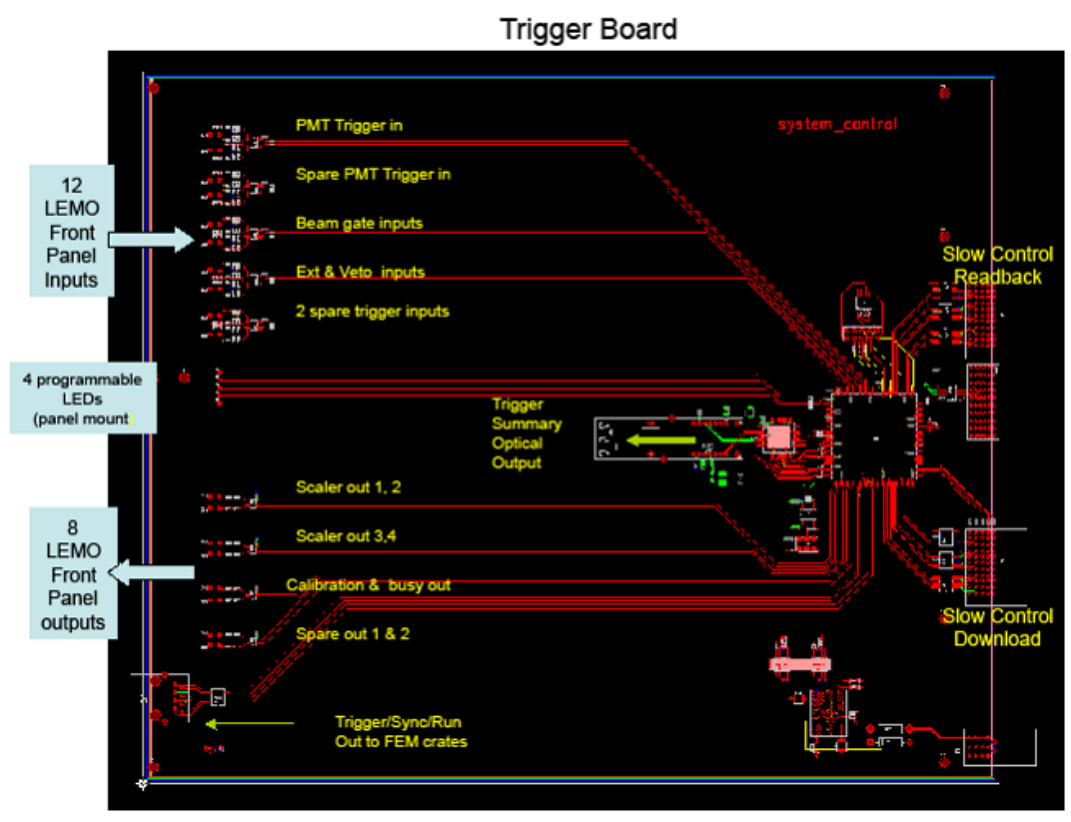

Figure 10.30 Schematic of MicroBooNE trigger board.

Each trigger condition receives a code ranging from 1 to $n$, with $n$ likely not exceeding 7 , and 0 meaning no trigger. This code transmits serially one bit at a time to the TB on one cable as shown in Figure 10.31. A second cable carries a marker to identify the first bit of a trigger code transmission.

Booster and NUMI beam gates also input to the TB, where they can be placed in coincidence with PMT triggers to flag neutrino interaction candidates. Valid PMT triggers are placed in a logical OR with other utility triggers sent to the trigger board to form calibration triggers for the electronics, random triggers for noise measurement, off-beam triggers for cosmic ray response studies, and others as deemed necessary.

All inputs to the trigger board (PMT or otherwise) will be via front panel LEMO connectors, as will the trigger output. Figure 10.32 diagrams the PMT readout and trigger logic.

An OR of all triggers passes to a fan-out module on a single cable, and from there distributes to all crate controllers (TPC and PMT), and through the crates backplane dataway to the FEMs. An FEM that receives a trigger inhibits its supernova readout mode with its associated decimation, initiates the finer-grained readout scheme and directs the data to the appropriate readout path.

Activation or masking of each of the trigger modes will be computer controlled as will the setting of the various trigger thresholds and conditions. The actual cause of the trigger will be 
available at the event building stage and off-line, as this information will be read out as a bit pattern from the TB.

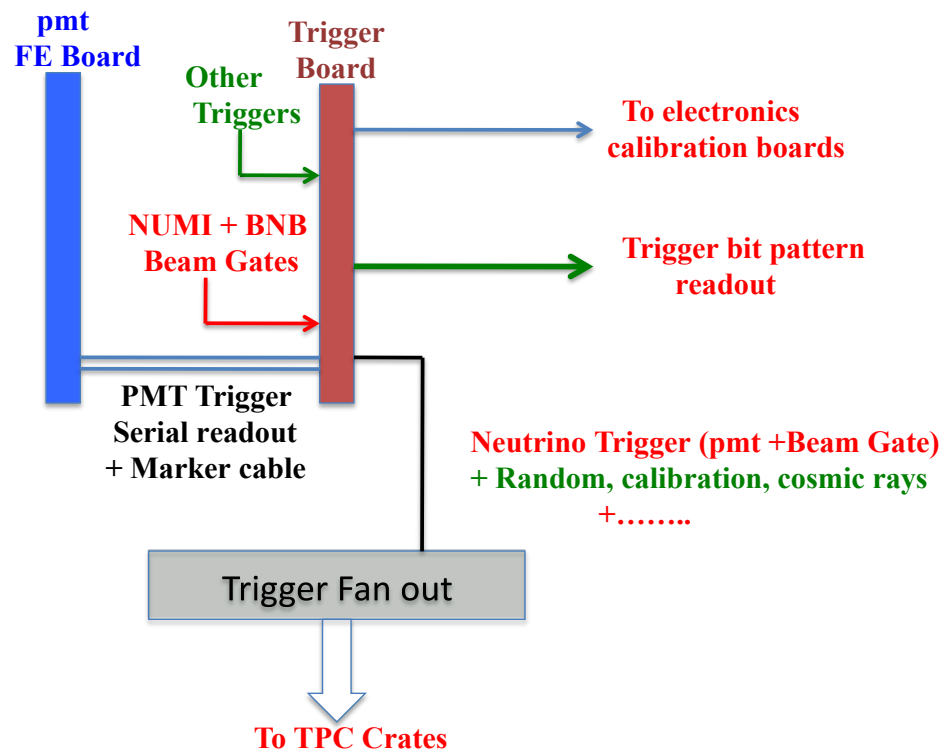

Figure 10.31 The modules and their interconnections used in the trigger logic.

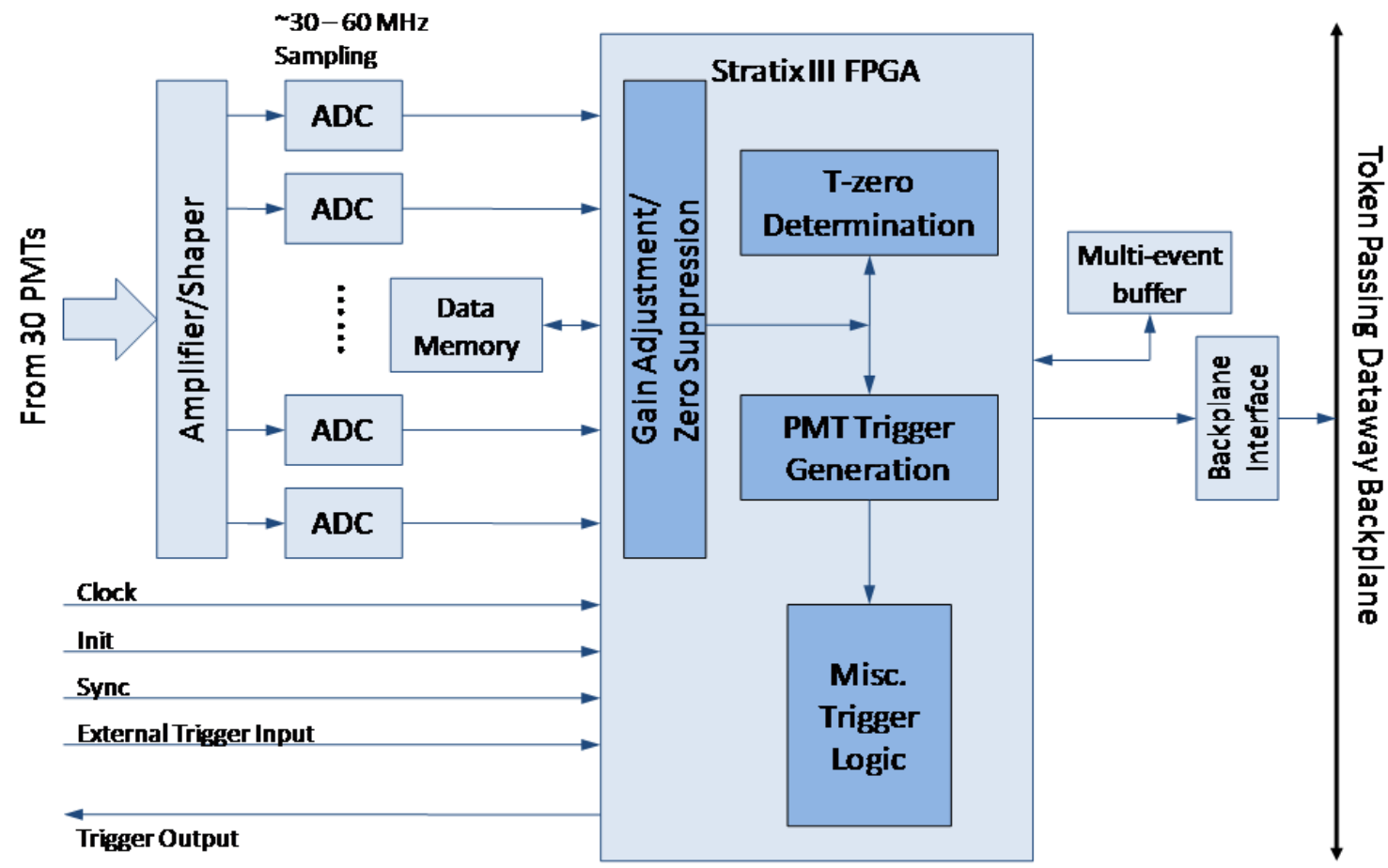

Figure 10.32 PMT readout and trigger logic. 


\subsection{Data Acquisition System (WBS 1.9)}

\subsubsection{Introduction}

The data acquisition system (DAQ) receives and buffers the data from both the TPC and the PMT readout electronics systems, and then builds and records the event based on the trigger decision. The DAQ also provides additional functionalities for run control, monitoring, system operations, and alarms. Both TPC and PMT readout electronics systems organize into readout crates mounted on racks installed in the detector hall. Each readout crate contains one transmit module (XMIT) to collect data from the TPC or PMT readout boards through the backplane and to transmit data to a DAQ PC (one per crate) through fiber optic links and a PCI Express card. The DAQ utilizes ten readout crates, and therefore ten transmit modules, ten PCs, and 31 PCI Express cards for the entire detector. Table 10.2 summarizes the main DAQ processes and the computers that they run on.

\section{Processes by Resource}

\begin{tabular}{|c|c|c|c|}
\hline Process & Description & Machine & Comment \\
\hline Assembler & $\begin{array}{l}\text { Starts Run. Builds Triggered } \\
\text { Events from SEB nodes. }\end{array}$ & uBDPC & $\begin{array}{l}\text { connects/listens to Sender, } \\
\text { issues StartSHM }\end{array}$ \\
\hline DaqLogd & $\begin{array}{l}\text { daemon to which all DAQ } \\
\text { processes declare state }\end{array}$ & uBDPC & daemon \\
\hline StartSHM & $\begin{array}{l}\text { Create shared memory; } \\
\text { connected to by most uBDPC } \\
\text { processes }\end{array}$ & uBDPC & $\begin{array}{l}\text { Chunk of memory of fixed } \\
\text { size for storing run state. }\end{array}$ \\
\hline Streamer & $\begin{array}{l}\text { Write Built Event to } \\
\text { appropriate sub-run file. }\end{array}$ & uBDPC & \\
\hline SNAssembler & $\begin{array}{l}\text { pre-scaled SN event builder. } \\
\text { Watches SNewsDaemon }\end{array}$ & uBDPC & $\begin{array}{l}\text { On a cron schedule to run } \\
\text { once/week }\end{array}$ \\
\hline SNewsDaemon & watches for SNEWs email & uBDPC & daemon \\
\hline ManageFileDB & Maintain File database & uBDPC & \\
\hline EPICS_IOC & $\begin{array}{l}\text { Read temperature, power } \\
\text { consumption, state, etc, of } \\
\text { this machine, Crate status }\end{array}$ & uBDPC & \\
\hline EPICS_shm_IOC & $\begin{array}{l}\text { Read shared memory of this } \\
\text { machine }\end{array}$ & uBDPC & \\
\hline Sender & $\begin{array}{l}\text { Send Triggered data over } \\
\text { sockets to Assembler }\end{array}$ & SEBPC.TPC & connects to Assembler \\
\hline WriteSNData & $\begin{array}{l}\text { Directly write SN evt buffers } \\
\text { to local disk }\end{array}$ & SEBPC.TPC & \\
\hline TriggeredDataRead & $\begin{array}{l}\text { Read Data in over devoted } \\
\text { TrigData PCle XMIT lanes }\end{array}$ & SEBPC.TPC & thread \\
\hline SNDataRead & $\begin{array}{l}\text { Read SNData in over devoted } \\
\text { SN PCle XMIT lanes }\end{array}$ & SEBPC.TPC & thread \\
\hline FPGAWrite & $\begin{array}{l}\text { FPGA Commands out over } \\
\text { PCle Controller card lanes }\end{array}$ & SEBPC.TPC & \\
\hline FPGARead & $\begin{array}{l}\text { FPGA data in over PCIE } \\
\text { Controller card lanes }\end{array}$ & SEBPC.TPC & \\
\hline
\end{tabular}

The MicroBooNE TDR (2/24/2012-DocDB 1821-v12): Electronics/Data Acquisition (WBS 1.5/1.9) Page 122 


\begin{tabular}{|c|c|c|c|}
\hline CalibrationRead & $\begin{array}{l}\text { Devoted Process between } \\
\text { runs reads over PCle XMIT } \\
\text { lanes }\end{array}$ & SEBPC.TPC & $\begin{array}{l}\text { properly timed with Calibrate } \\
\text { on SEBPC.PMT }\end{array}$ \\
\hline SuperNovaReaper & $\begin{array}{l}\text { Monitor SN data on SEB local } \\
\text { disks }\end{array}$ & SEBPC.TPC & \\
\hline EPICS_IOC & $\begin{array}{l}\text { Read temperature, power } \\
\text { consumption, state, etc, of } \\
\text { this machine }\end{array}$ & SEBPC.TPC & \\
\hline Calibrate & $\begin{array}{l}\text { Inject charge over PCle } \\
\text { controller lanes }\end{array}$ & SEBPC.PMT & \\
\hline * & $\begin{array}{l}\text { Instances of all above } \\
\text { processes on SEBPC.TPC, } \\
\text { applied here to PMT data }\end{array}$ & SEBPC.PMT & \\
\hline ReadACNETData & Get Accelerator data & uBNPC & \\
\hline ReadTriggeredData & $\begin{array}{l}\text { Read the assembled evts } \\
\text { from file }\end{array}$ & uBNPC & \\
\hline EPICS_Mon & Read select Slow Mon data & uBNPC & \\
\hline CosmicsTagger & $\begin{array}{l}\text { Apply a simple cosmic muon } \\
\text { filter to tag event as to } \\
\text { likelihood that it's a cosmic } \\
\text { event }\end{array}$ & uBNPC & \\
\hline SplitterWriter & $\begin{array}{l}\text { Write to individual file } \\
\text { streams, per trigger header } \\
\text { and taggers }\end{array}$ & uBNPC & \\
\hline NearlineChecks & $\begin{array}{l}\text { Monitor data with ROOT } \\
\text { processes, write to run status } \\
\text { histograms. Keep reasonable } \\
\text { time histories for watching } \\
\text { trends. }\end{array}$ & uBNPC & \\
\hline MoveFilesToAFS & $\begin{array}{l}\text { Move files around, particularly } \\
\text { files to be viewed for Shifter } \\
\text { monitoring to web server- } \\
\text { viewable area. }\end{array}$ & uBNPC & \\
\hline Enstore & $\begin{array}{l}\text { Back up data stream files to } \\
\text { Enstore }\end{array}$ & uBNPC & \\
\hline ManageFileDB & Maintain File database & uBNPC & \\
\hline EPICS_IOC & $\begin{array}{l}\text { Read temperature, power } \\
\text { consumption, state, etc, of } \\
\text { this machine }\end{array}$ & uBNPC & \\
\hline MetaEPICS & $\begin{array}{l}\text { Push EPICS data to one } \\
\text { place at appropriate } \\
\text { decimation. Back-up this data } \\
\text { to Enstore storage. }\end{array}$ & uBNPC & \\
\hline Reconstruction & pre-scaled reconstruction & uBNPC & as machine resources allow \\
\hline EvtDisplay & pre-scaled Event Display & uBNPC & as machine resources allow \\
\hline
\end{tabular}

Table 10.2 Summary of data acquisition processes for MicroBooNE.

The MicroBooNE TDR (2/24/2012-DocDB 1821-v12): Electronics/Data Acquisition (WBS 1.5/1.9) Page 123 


\subsubsection{Technical Design Criteria}

The MicroBooNE detector operates in two primary modes, accelerator and supernova, each with its own data stream. The following list summarizes main design criteria for the DAQ system:

- Accelerator stream: data rate $\sim 1.2 \mathrm{MB} / \mathrm{s}$ per crate with data gathered from ten digitization crates, and events built and stored from crate data.

- Supernova stream: data rate $\sim 50 \mathrm{MB} / \mathrm{s}$ per crate to the DAQ, much less to permanent storage, with data queued until expiration or receipt of a supernova trigger.

- Data management: binary format data storage with file management, registration, and accountability.

- Monitoring: DAQ provided data and detector monitoring in real time.

- Run control: DAQ provided run control including user interface, run stop and start, system integrity checking, and error recovery.

\subsubsection{DAQ Event Building Electronics}

Sixteen FEM cards group into a crate that also houses an XMIT module in its seventeenth slot. The latter transmits the data to PCs and then, for supernova data, onto hard drives for permanent storage. One PC (the sub-event PC, or SEB PC) connects to each crate. The crate additionally houses a crate controller needed to read and write parameters and information from the FEM and XMIT. The DAQ needs nine crates and nine PCs to read the 8256 TPC wires and process their information. An additional crate and PC processes PMT information.

Each XMIT module includes four optical links, two used for the accelerator neutrino data stream and the other two for the supernova data stream. These links connect to optical transceivers housed on two PC-resident PCI Express interface cards developed at Nevis for use in ATLAS. The two transceivers on the each interface cards handle $6.4 \mathrm{~GB} / \mathrm{s}$ traffic and connect to a 4-lane PCI Express bus, with each lane accommodating $2.5 \mathrm{~GB} / \mathrm{sec}$. The XMIT module transmits data to the PC based on the token passing technique shown in Figure 10.33. The XMIT module generates the token and passes to the first FEM module. This module receives the token and transmits its data, if any. The data transits from one board to the next on the crate backplane until it reaches the XMIT module. The token passes to the next FEM module when an active FEM finishes transferring its data. Since a module only drives the data to its neighbor, it forms a pointto-point short link. Data can flow at rates up to $512 \mathrm{MB} / \mathrm{sec}$ on this path, but the expected traffic on this dataway should be a factor ten slower.

Figure 10.34 shows the layout of the crate backplane. In addition to the token passing dataway, a secondary bus serves to download the FPGA codes and initiate data/parameter readback via the crate controller and a third PCI Express card in the PC. This slower readback can be used to read out the FEM without the XMIT, a useful feature for system development, for operating a lightweight test stand, and for other purposes. 
Each PC stores supernova data in disk drives that can accommodate $100 \mathrm{MB} / \mathrm{sec}$ writing speeds, but for which the DAQ design assumes a more conservative $50 \mathrm{MB} / \mathrm{sec}$. An additional PC collects the data from the ten sub-event PCs and builds accelerator events.

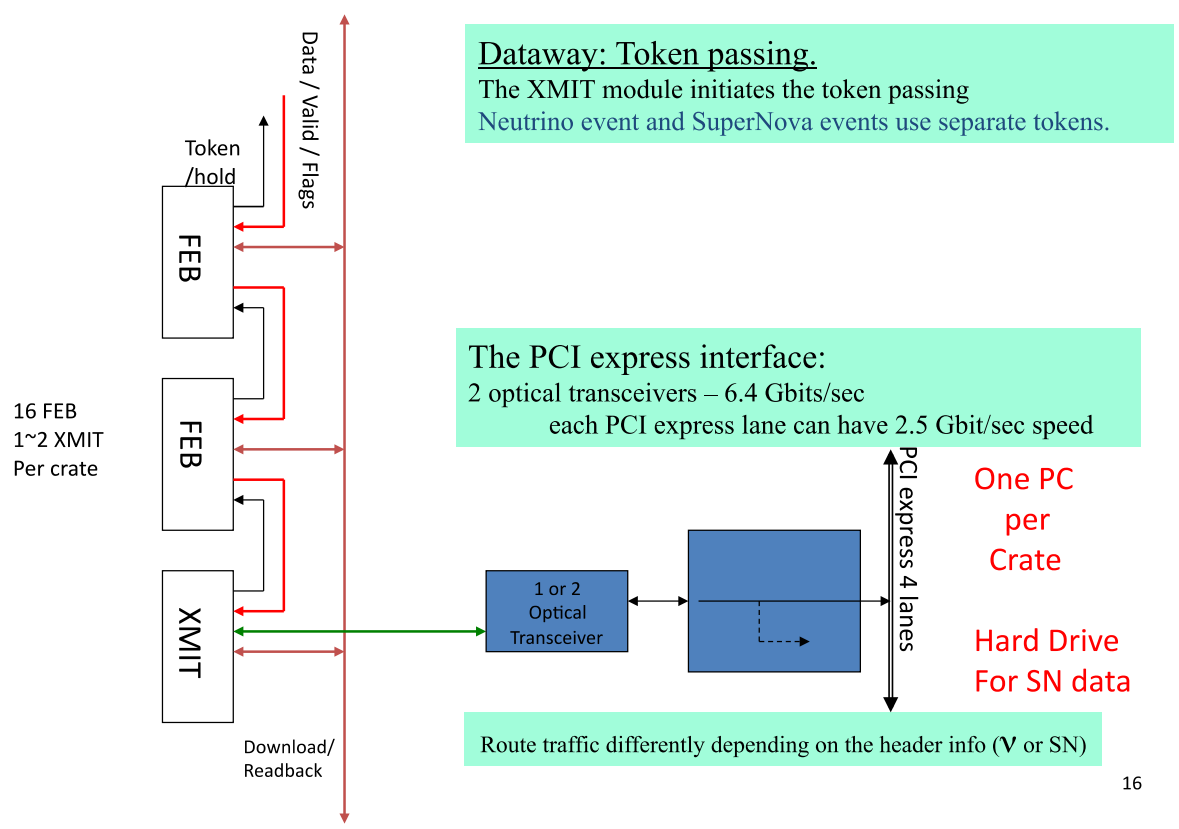

Figure 10.33 The token-passing scheme for data transmission from the FEB's to the PC's via the crate backplane dataway and the PC resident PCI Express cards.

\section{Crate Backplane}

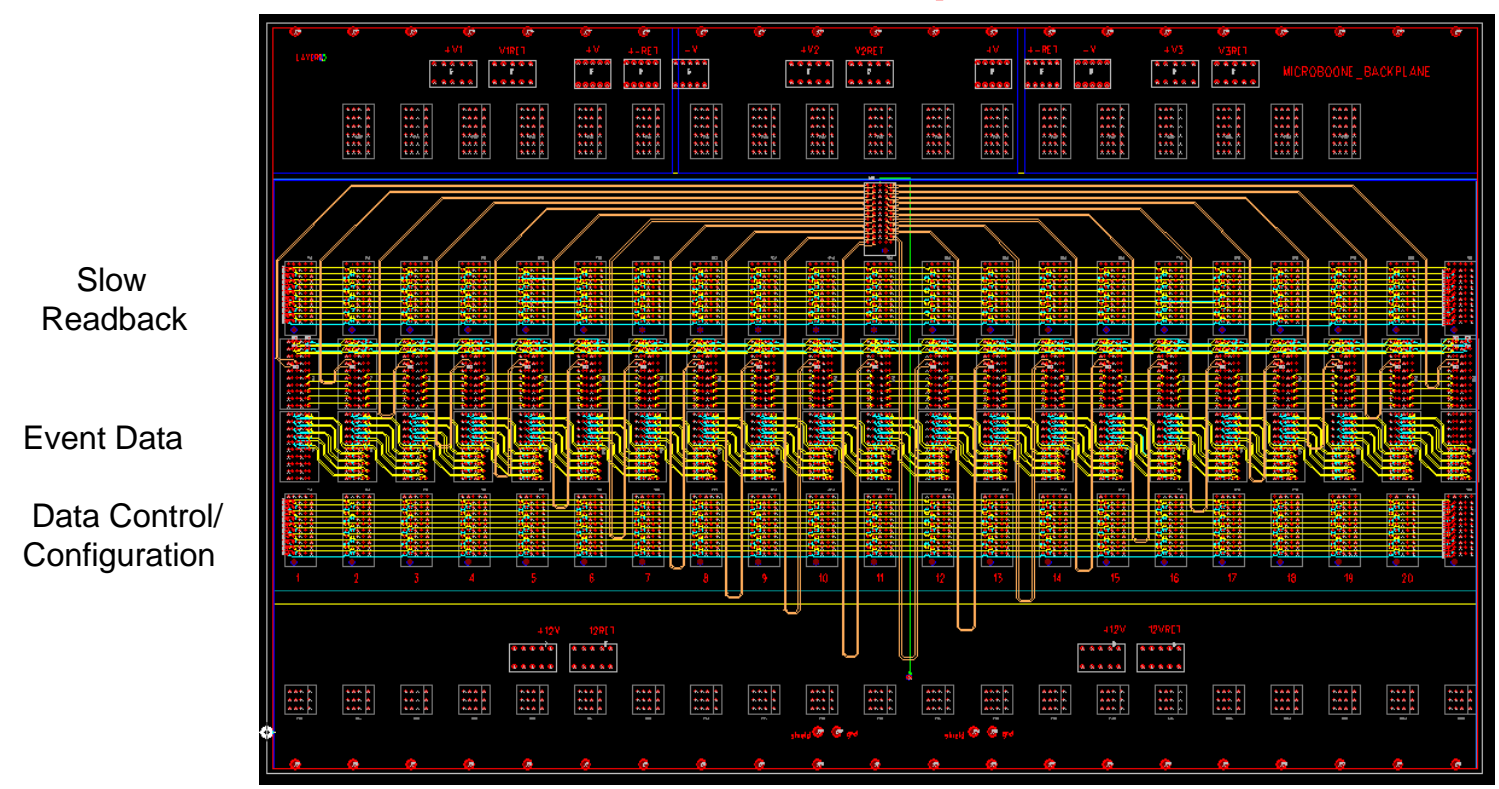

Figure 10.34 Layout of the crate backplane. 


\subsubsection{Data Volume and Rates}

The DAQ reads $4.8 \mathrm{~ms}$ of data for each accelerator event. This translates to a raw data rate per crate per trigger of $4.8 \mathrm{~ms} \times 2 \mathrm{MHz} \times 1000$ wires $\times 2$ bytes per word $=19.2 \mathrm{MB} / \mathrm{event}$. Assuming a worst case in which every BNB spill generates a trigger, the $10 \mathrm{~Hz}$ neutrino beam rate with a factor ten expected compression yields a maximum data rate of $19.2 \mathrm{MB} / \mathrm{sec}$ per optical link, still below the maximum disk writing speed. The actual rate of beam neutrino events should be only one per several hundred seconds. Cosmic rays pass through the MicroBooNE detector at an expected rate of $3 \mathrm{kHz}$. From this, the number of cosmic ray events per $1.6 \mu$ s accelerator beam spill can be estimated as $\left(3000 \times 1.6 \times 10^{-6}\right)=4.8 \times 10^{-3}$. This gives a rate of one cosmic ray event per 208 spills, or one per $21 \mathrm{~s}$. Cosmic rays thus comprise the dominant source of events triggered by the PMT system in coincidence with the beam spill.

The $50 \mathrm{MB} / \mathrm{sec}$ assumed disk writing speed governs the compression ratio needed to be applied to the supernova triggers, estimated as $50 \mathrm{MB} / \mathrm{sec} /[1 \mathrm{crate} / \mathrm{PC} \times 2 \mathrm{MHz}$ sampling rate $\times 1000$ wires $/$ crate $\times 2$ Bytes] $=1 / 80$. This compression ratio of $1 / 80$ can be reduced further in a number of ways, for example, decimation to $1 \mathrm{MHz}$, increasing the number of PCs, or increasing the number of disk drives. The DAQ continuously stores the supernova data in a circular buffer such that the last two days' data remain available should a supernova alert be received.

\subsubsection{DAQ System Architecture}

The MicroBooNE event building and process flow model follows the successful MiniBooNE DAQ implementation. Neutrino events in MicroBooNE arrive from the BNB at the same frequency as at MiniBooNE. Thus, despite the factor of ten more channels in MicroBooNE, the factor of two lower sampling rate in the MicroBooNE TPC, and the longer sampling windows due to the $3 \times 1.6$ millisecond drift time width, much of the code and the process architecture can be readily adapted from MiniBooNE.

All crates receive a common $64 \mathrm{MHz}$ clock pulse. The PMT FEM samples its ADCs at that rate, while the TPC FEM reduce the clock frequency internally to $2 \mathrm{MHz}$, thus sampling each wire input channel once every 500 ns. The TPC crates receive the output of the trigger board, consisting of an OR of all possible PMT plus beam gate triggers and any other utility triggers through their crate controllers. The crates also receive the $1.6 \mu$ s wide BNB trigger at a maximum rate of $15 \mathrm{~Hz}$, and the Fermilab Main Injector NuMI beam trigger at a $0.5 \mathrm{~Hz}$ rate. Charge-injection calibration signals also go into each crate after being split.

The appropriate logic among these signals creates triggers for the electronics that result ultimately in data appearing at known memory locations in the sub-event buffer (SEB) PCs' DMAs. The SEB PC DMAs establish the point in the system where the DAQ assumes control of the data. As well as transferring data from the SEB PC DMAs, the DAQ can give instructions that fill the DMAs and initiate controls back at the electronics, e.g., commands for clearing registers or changing FPGA coding. The SEB PCIe cards carry out this communication with the DMA cards via their drivers. Producing the $C$-functions for these drivers to pull desired memory 
contents out of the SEB PCs' DMAs for use in the DAQ readout program running on the SEB PC constitutes a critical task in the early phase of DAQ development.

The SEB PCs alert the main MicroBooNE DAQ computer uBDPC to the existence of data in the appropriate registers of the SEB PCs' DMAs. For a supernova event, the SEBs write data to local disks and clear the DMAs. The supernova data reside on disk in a few-day duration circular buffer that gets overwritten when full. Two 2 TB drives fill in 24 hours on each SEB PC with supernova data. The main uBDPC unit queries and builds events from supernova data files on the ten disks at a low ( daily) pre-sample rate. If a delayed, real supernova trigger from SNEWS arrives, uBDPC pulls data from the disks, and builds events to be passed down the pipeline for further processing, exactly as with the pre-sample event building. The SNEWS signal generates a uniquely keyed e-mail watched for by a process running on uBDPC. It should arrive within 20 minutes of an initial indication of a supernova from the worldwide SNEWS detector network.

For triggered events, uBDPC pulls data from the SEB PCs via a TCP/IP socket protocol. It assembles these data with the correct $500 \mathrm{~ns}$ timestamps within the $4.8 \mathrm{~ms}$ window into one event. Then uBDPC assembles the data from each SEB PC, adds a header according to the initiating trigger, time stamps the event, performs other validation functions, and finally writes the event it to its disk.

Near-online ("nearline") processes run on their own dedicated machines, referred to as uBNPC. Use of up to two uBNPC allows for load sharing. These processes concatenate beam, slowcontrols, and monitoring information, adding further headers as necessary. Separate physics streams can thus be created with the nearline processes through examining the trigger bits accompanying an event, or by running a "lightweight" version of offline reconstruction software on uBNPC. For example, cosmic ray events or accelerator events containing only through-going muons could be reconstructed using the simple two-dimensional tracking described in Chapter 12.

An error daemon also runs on uBDPC for message logging from all processes in the DAQ computation farm. All online and nearline processes connect and write to this daemon. The daemon writes to a file containing all error and state information for further querying.

\subsubsection{DAQ Application Tasks}

This section provides more details on processes that run on the SEB PCs, one of which serves each crate; uBDPC, the main DAQ machine; and the nearline machine uBNPC. Figure 10.35 provides a diagram of DAQ application tasks.

\subsubsection{SEB PC processes}

A continuous trigger loop process runs on each SEB PC, watching volatile trigger memory that becomes non-empty when crate hardware sets a trigger. The SEB PCs then query the relevant locations in the DMA for non-null data. 
Another process runs on the SEB PC called Sender. The trigger process instructs Sender about which DMA addresses it should point to and how much data it should grab from DMA. This process runs in constant TCP/IP socket connection with the assembler process, called Assembler, on uBDPC. Each Sender informs Assembler when it has data and then ships the data to Assembler.

\subsubsection{2 иВDPC}

This main DAQ computer first starts a daemon to listen for DAQ log connections from all possible processes in the DAQ. It logs error and normal operation messages, redirect these data at regular intervals to a file. Signal handling messages go here for state recovery so that if the assembler process dies, it can be restarted and reconnected without loss. Mutex locking ensures only one process connects at a time, preventing the error daemon from being written to simultaneously by many processes.

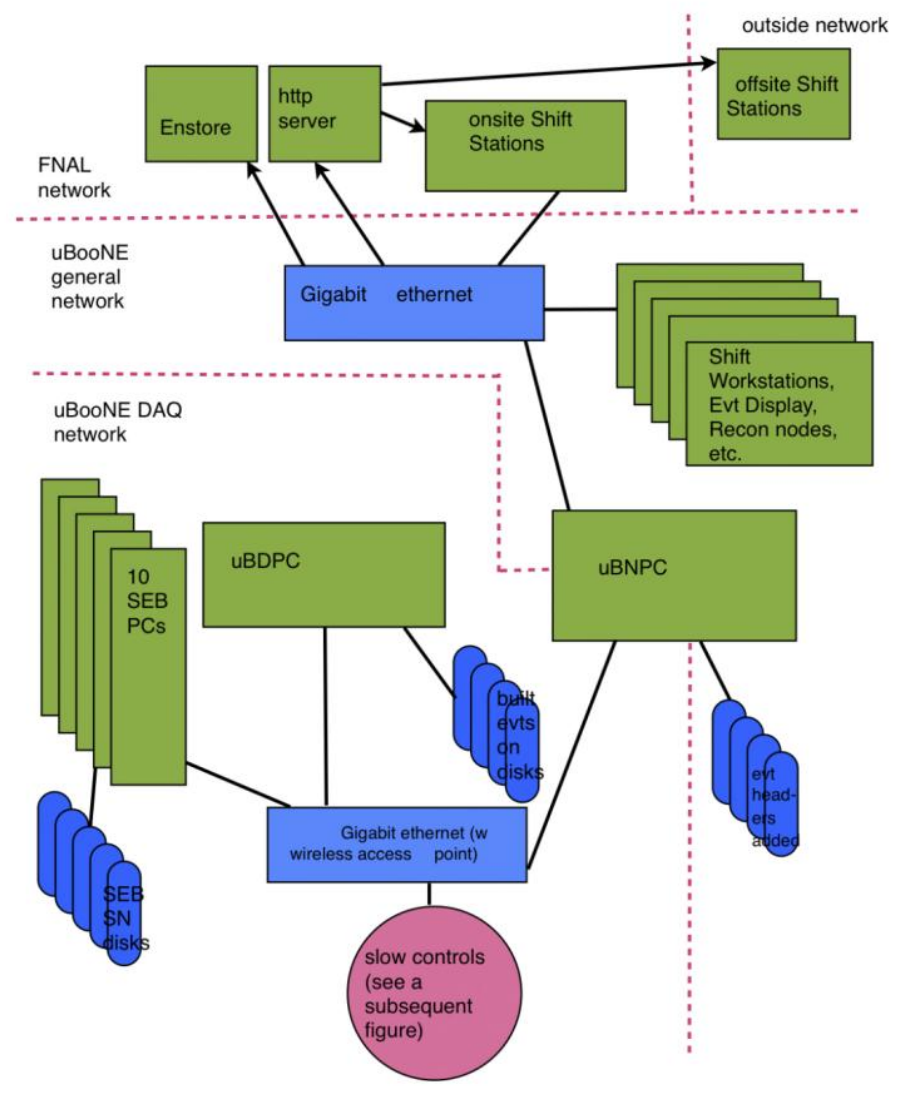

Figure 10.35 Diagram of DAQ application tasks. 
Assembler is the main process for the DAQ running on uBDPC. It starts up the shared memory over which it remains in exclusive control. It maintains snapshots of various DAQ state parameters. Processes running on uBDPC display this information to a text screen, and dump it to a file for outside client viewing via a GUI. Assembler's main task consists of building events. It connects to the Sender daemons that control the SEB data, aligning wire and PMT data by timestamp at the finest (25 ns for the PMT) timescale, and putting together the $4.8 \mathrm{~ms}$ events. Data sparsification methods such as the lossless Huffman compression algorithms described previously reduce event sizes to approximately $40 \mathrm{MB}$, and Assembler holds these fully built events in memory.

The uBDPC process Streamer communicates with Assembler by a socket connection. It takes the built data, adds a header, and writes it to local disk in binary (".fc") format at a rate of less than $1 \mathrm{MB} / \mathrm{sec}$. At this rate, Streamer writes $0.1 \mathrm{~TB}$ per day, filling one $2 \mathrm{~TB}$ disk every twentieth day. MicroBooNE will dedicate at least two drives for Streamer output.

Cosmic ray muons drive the accelerator mode data rate, with neutrino events from the beam occurring only once every ten minutes. This low rate allows for the possibility of recording events taken with random or very low-bias trigger for detector performance studies; the extra rate from these triggers would negligibly perturb neutrino data taking. For example, a "strobe" trigger may be fired randomly outside of the beam gate at a few $\mathrm{Hz}$ for detector studies. A special trigger with a reduced threshold on number of PMT hits may similarly be desirable during commissioning and maintenance functions during operations. The available data volume for these special triggers could be as large as ten times that expected from cosmic ray events. A simple nearline process could monitor and dump most of this stream before permanent storage.

Assembler forks a daemon for accepting incoming remote commands. This daemon negotiates, among other things, the socket connections to the SEB PCs, which also run instances of this daemon.

\subsubsection{3 uBNPC}

Nearline processes run on uBNPC that read the binary ffc files from the uBDPC disk, add headers, and merge ACNET and EPICS data, all the while preserving and saving the raw .fc files. They also split the file into physics streams determined by simple event topologies and trigger information. A ROOT process runs on $\mathrm{UBNPC}$ that writes each data stream to its own .root file. Files get their md5sums (the industrial standard file "fingerprint") tacked on for integrity checks at analysis time. A series of 2 TB disks in a RAID configuration holds these files. The Fermilab CD's ENSTORE process operates on one of these particular drives and backs up the data streams. The files then move down the chain of drives until upon space remains. This first disk must be large enough to hold data for few-day ENSTORE connection or operation outages. Two TB provide sufficient capability for four days of storage, but many more will be available. 
Devoted nearline processes also read heavily pre-scaled supernova event files, build events from the raw SEB PC disk data, and save events to files. This allows a robustness check on the supernova data compression and provides the opportunity to practice for a real SNEWS trigger.

The uBNPC PC additionally runs processes that create online or nearline files for the MicroBooNE web server, accessible via AFS. Client browsers view these files, producing displays such as histograms of wire occupancy and PMT ADC counts, and card and crate ensemble statistics. A python script running a ROOT job can access a several-week history of .root files and generate time series data to be viewed by a client browser to help reveal detector trends that might otherwise escape unnoticed.

Nearline reconstruction may run locally on uBNPC as the experiment is commissioned, subject to CPU demands, or on supplemental machines that can access uBNPC disks, likely in arrays of condor jobs. These $1 \mathrm{U}$ machines can be added to the uBNPC rack as they become available. Event displays can run from files of interest on uBNPC, and image files (.jpg, .png, .pdf, etc.) from the LArSoft event display running as a nearline process can be saved to the AFS-mounted web server directories for widespread perusal starting at an early date in the commissioning of MicroBooNE.

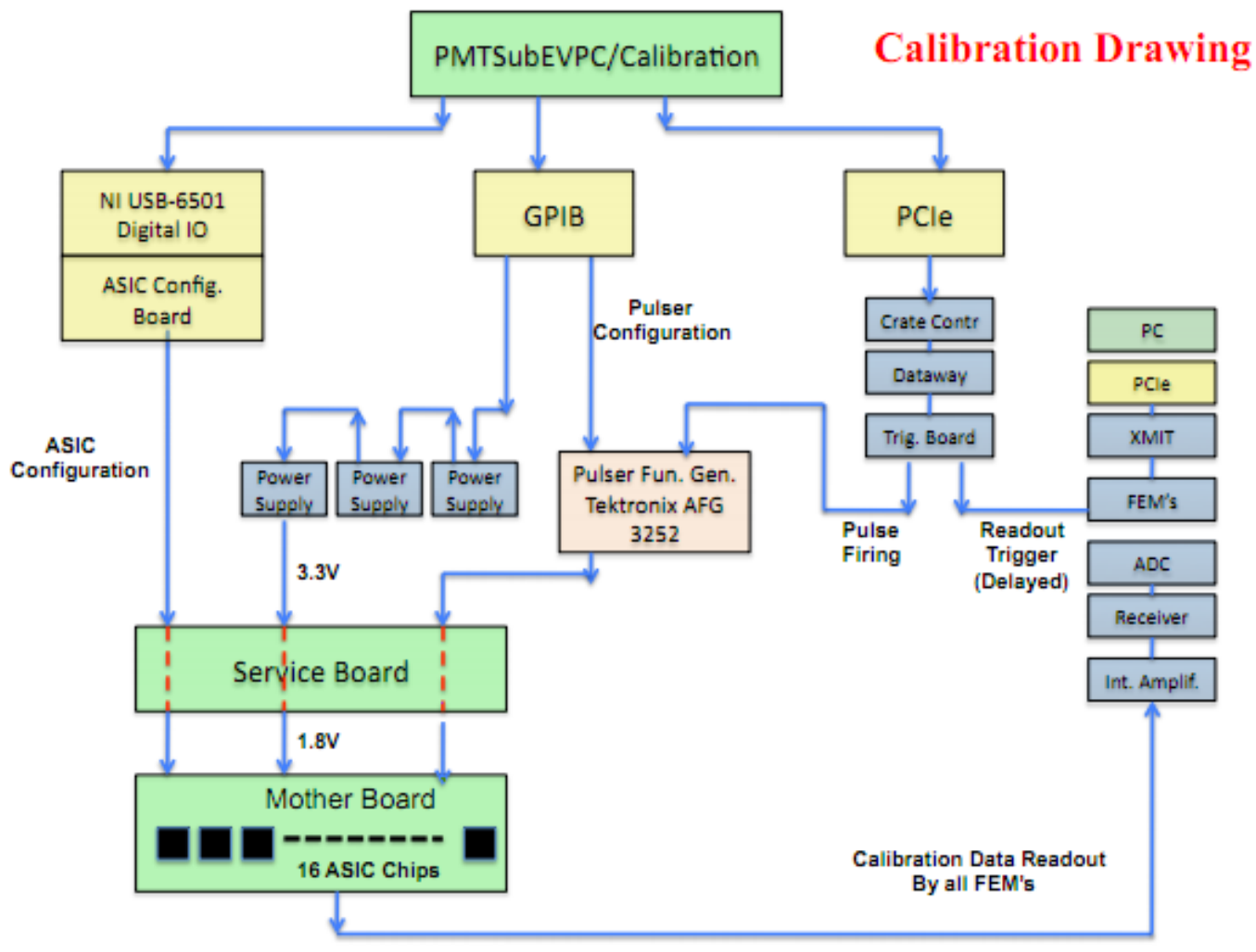

Figure 10.36 DAQ integration test procedure schematic. 


\subsubsection{DAQ Integration Test with TPC Calibration}

A calibration run will be performed with an empty cryostat to validate the integration of the DAQ with other MicroBooNE detector elements prior to data taking. Figure 10.36 shows a schematic of the calibration components.

The calibration run consists of the following steps: (1) The uBDPC connects to the SEB PCs over sockets, in the way previously described. The uBDPC passes instructions to start a calibration run. Supernova data-taking processes do not start in this run mode. (2) The PMT SEB PC configures the peaking time of the ASICs through the National Instruments digital IO unit and ASIC configuration board and service board. (3) The PMT SEB PC configures the Tektronix AFG3252 function generator for the pulse height, rise/fall time, pulse duration, and pulse mode. (4) The TPC SEB PCs initialize and set up controller cards and load the SEB PC XMIT file paths and parameters, as well as the FEM FPGA and FEM parameters at this point. (5) All SEB PCs set up the XMIT PCIe DMA preparation. (6) The PMT SEB PC then tells its controller card to instruct the trigger board to tell the Tektronix pulser to fire. Simultaneously, with appropriate delay, the trigger board, via a control card, sets the trigger word's calibration trigger bits. Other triggers will likely be suppressed for in this process. (7) These actions cause an event ( $3 \times 1.6 \mathrm{msec}$ frames) at all crates, which will be read in the normal way via DMA from the XMIT cards.

\subsubsection{Detector Control and Monitoring}

\subsubsection{Slow Controls and Monitoring}

The MicroBooNE slow-controls system can be accessed and monitored from any client PC on the MicroBooNE DAQ network. A client user queries the top level EPICS database on the channel access server. Run management through slow controls normally takes place on uBDPC, but it can be initiated from other machines as well.

Figure 10.37 outlines the design of the slow controls and monitoring system. Input/output controllers or drivers (IOCs) operate on PCs running EPICS. Three 1U rack-mounted Linux PCs comprise this system. The first PC controls the $\sim 100$ high voltage channels, the second manages the $\sim 70$ low voltage channels, and the third controls a National Instruments bus that connects to temperature and pressure probes, vacuum gauges, interlocks, and other environmental monitors. Use of the National Instruments (NI) bus requires interfaces between NI software and our Linuxbased EPICS control system, a feat which has been accomplished in other experiments. Also under consideration is a potentially superior design based on small, embeddable, networkenabled microcontrollers and single board computers (SBC) which run Linux directly. An example of a suitable SBC is the Glomation SBC 9G20, which is comparable in size to the Netburner microcontrollers used for some slow monitoring in MINERvA ${ }^{1}$.

The slow controls system requires one National Instruments bus per roughly 64 channels. Each of the PCs used in the system connects to a Gigabit switch, into which each piece of hardware to

The MicroBooNE TDR (2/24/2012-DocDB 1821-v12): Electronics/Data Acquisition (WBS 1.5/1.9) Page 131 
be controlled or monitored also connects. The SEB PCs can also run EPICS at low overhead that do not interfere with their TPC/PMT data-taking tasks. These SEB PC instances of EPICS control and monitor the SEB voltage and current readings in the crates via the PCIe card. The IOCs pass on the HV, LV, temperature, pressure, and the like to their local database; or they read from this database. Each EPICS PC stores its settings, readings, and alarm conditions in a database. All EPICS PCs report to a master EPICS instance running on an independent PC. This database maps the desired IOC device to the proper PC and corresponding local database, and finally, pushes coarsely time averaged quantities at a low rate (once per 1-2 hours) to a database on the FNAL network.

Table 10.3 lists elements of slow control and monitoring system.

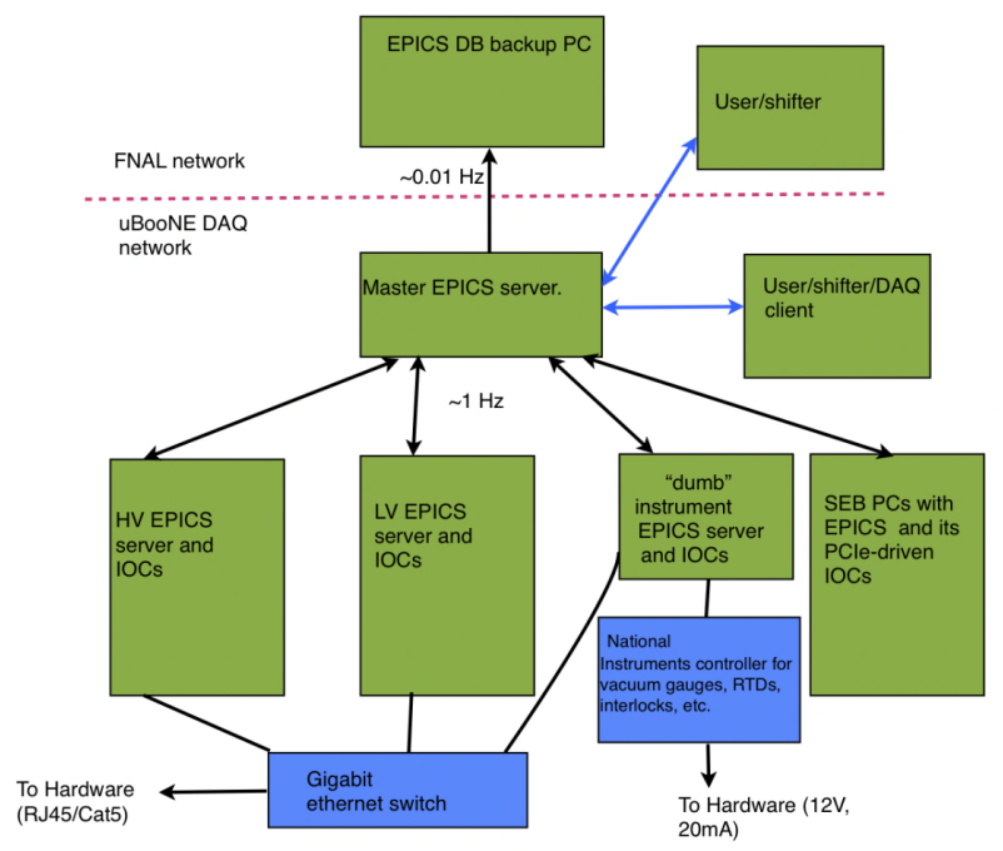

Figure 10.37 Diagram of slow control and monitoring.

The MicroBooNE TDR (2/24/2012-DocDB 1821-v12): Electronics/Data Acquisition (WBS 1.5/1.9) Page 132 


\begin{tabular}{|c|c|c|c|c|c|}
\hline System & no. & no. & values & Total & Notes \\
\hline LVPS - rails & 7 & 3 & $\times 4$ & 84 & $\begin{array}{l}\text { Voltage, current, state of power supply rails for on- } \\
\text { detector service boards }\end{array}$ \\
\hline LVPS - units & 7 & & 6 & 42 & Status of power supplies for on-detector service boards \\
\hline Rack temp. & 13 & & 1 & 13 & Temperature monitoring device mounted in each rack \\
\hline MVPS - bias & 1 & 3 & $\times 2$ & 6 & Voltage, current, state for $-204 \mathrm{~V},+440 \mathrm{~V}$ \\
\hline HVPS - TPC & 1 & & 3 & 3 & Voltage, current, state for $150 \mathrm{kV}$ PS \\
\hline HVPS - PMT ch. & 40 & & 3 & 120 & Voltage, current, state for each PMT HV channel \\
\hline HVPS - PMT units & 8 & 3 & $\times 2$ & 48 & Voltage, current for PMT HV motherboards \\
\hline DAQ PC status & 17 & & 3 & 51 & Power status, temp., CPU load. \\
\hline Fast DAQ status & 1 & & 8 & 8 & Run mode, run number, data rate, etc. \\
\hline Trigger status & 1 & & 8 & 8 & Rate and status of various trigger conditions. \\
\hline Calibration status & 11 & & 12 & 132 & Modes for the 12 pulses from calibration board. \\
\hline $\begin{array}{l}\text { Environment and } \\
\text { Operations }\end{array}$ & 16 & & 4 & 64 & Air temperature, humidity, pressure, AC power. \\
\hline Argon Purity Monitor & 3 & & 1 & 3 & Read only, no control. \\
\hline Cryogenic sensor data & 8 & & 1 & 8 & Press., temp., levels accessible via cryo control system \\
\hline TOTAL & & & & 590 & \\
\hline
\end{tabular}

Table 10.3 List of elements of slow control and monitoring.

The MicroBooNE TDR (2/24/2012-DocDB 1821-v12): Electronics/Data Acquisition (WBS 1.5/1.9) Page 133 


\subsubsection{Slow control run control}

In routine "warm run restart" mode, only the event building communications processes to the SEB PCs need to be restarted, and these restarts can safely be automated. The DAQ runs independently of the slow controls and monitoring. After appropriate checks on detector readiness and state checks, a script runs on uBDPC that ensures connection to and operability of the EPICS DBs. This script further checks for alarms or trip conditions, verifies nominal settings, and finally starts the run. A "cold run restart" may power up the electronics racks, reboot of the SEB PCs, and possibly a reboot of uBDPC before proceeding to the steps described for a warm restart.

Assembler reads EPICS data from its top database and writes a few words of information into the event header. It also reads ACNET data from Fermilab Accelerator Division-supplied pipes and sparingly incorporates this information into the event header as well.

\subsubsection{TPC/PMT run control}

The SEB PCs first run initialization scripts that include starting daemons that allow their communication with uBDPC. They then run scripts to start base PCIe functions on uBDPC that determine the readiness of the FPGAs, and then pass onto FPGAs read parameters, calibration constants, and other configuration data. Assuming no error conditions exist, uBDPC starts the rest of its daemons and initiates Assembler, starting the run. Run numbers increment per the run database.

If necessary, detector-specific databases may be maintained separately from EPICS, though synced to it occasionally; however, the preferred and natural choice entails doing everything within EPICS. The Fermilab CD service ENSTORE backs up the EPICS database periodically, maintaining its integrity and making it available for offline use.

A run opens a file that can grow to a few GB in size within about 15 minutes. Upon reaching this point the file closes and a new one opens, with the associated run number incrementing and updating in the database. Events update with each assembled event written to the file. Alternatively, sub-runs may be closed and opened every minute or so to keep individual file sizes down to more manageable size, with file concatenation taking place at run completion.

The run script continues the start/close/concatenate mode indefinitely until uBDPC receives a run stop or run pause command. Scripts running on the EPICS client PCs can initiate alarms that inhibit run start-up or end an ongoing run. These alarms get written to the logger daemons and signal the run shifter. Browser clients on remote shifter workstations display information accessed by the run logger daemon, combined with that from the shared memory file and the databases for EPICS and ACNET. Nearline processes running on uBNPC also provide data to the browser clients. 


\subsubsection{Racks and Other Infrastructure}

\subsubsection{DAQ racks}

Ten MicroBooNE crates installed in four racks reside on a platform above the cryostat. The nine TPC crates hold sixteen 64-channel FEBs per crate, as well as a crate controller and an XMIT card. The tenth crate holding the DAQ electronics for the PMTs contains one 64-channel FEM modified for PMT with a different digitizing section. A PC and an XMIT card and controller also sit in the PMT crate, as does the trigger board.

Four fiber optics cables connect each crate's XMIT cards to the SEB PCs. Two cables serve the accelerator neutrino event stream, and the other two dedicated to the continuously filling supernova buffers.

Ten 2U SEB PCs mount into a separate rack. This rack contains a $1 \mathrm{U} 24$ port gigabit Ethernet switch, to which the SEB PCs for both TPC and PMTs connect via CAT5 RJ45 cable. Three 1U, 4-bay, 2 TB SATA drives also reside in this rack, one for each SEB PC and two reserved for back up. As an alternative, one 4U 20-bay drive, with 2 TB in each unit may be RAID-configured to provide inherent backup for each drive. In any case, a half-day of supernova data stores onto these disks before being overwritten. Each TB drive fills with one day of compressed supernova data.

One or two 1U, rack mount keyboard monitors with an integral KVM switch provide detector hall access to each of the SEB PCs. One 2U, 4-core, 8+ GB memory Linux PC, the main MicroBooNE DAQ PC called uBDPC, sits in this rack and plugs into the 24-port gigabyte switch. The nearline machine uBNPC receives a similar configuration and resides in the same rack. Both major DAQ PCs have their own dedicated 1U rack-mount keyboard display for development. A 1U, 2×2 TB SATA RAID hangs off uBDPC. MicroBooNE may use larger drives for $\mathrm{uBDPC}$ if per-terabyte prices fall over the next two years. A PCI card on uBNPC allows hanging of order $2-4 \mathrm{U}$, RAID $2 \times 2$ TB drives, which would retain as much as a month of MicroBooNE data. At least 4 TB of writable disks will be available at startup. The uBNPC machine will plug into a different 16 or 24 port gigabit switch. This switch, and hence uBNPC, sees both the MicroBooNE detector network and the MicroBooNE general network, while uBDPC remains inside the MicroBooNE detector network. 


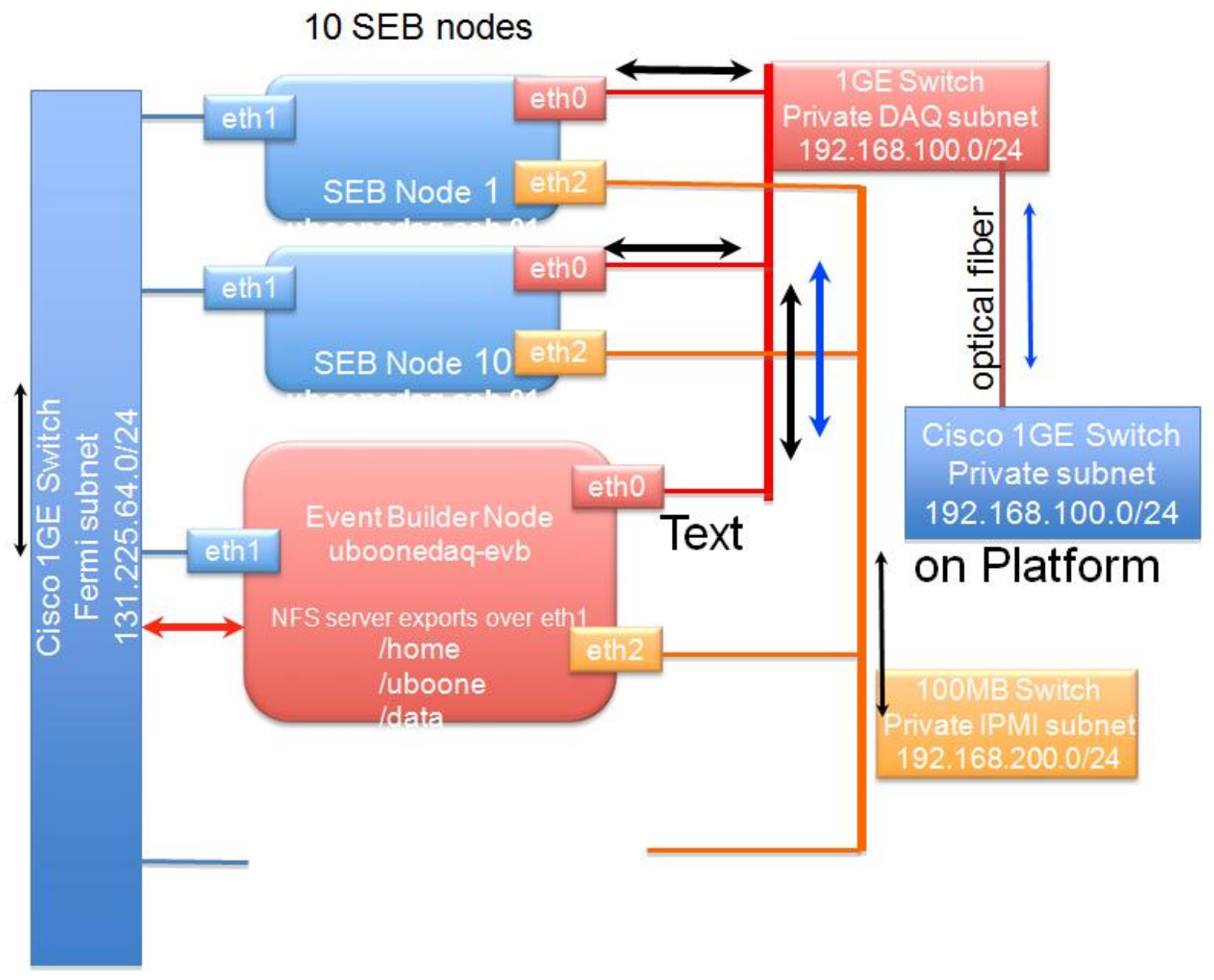

Figure 10.38 MicroBooNE network design.

\subsubsection{Slow Control/Monitoring Racks}

A separate electronics rack contains three 1U rack-mount Linux PCs for detector control. These run EPICS and the IOCs for the various hardware components. They plug into their own 1U 24 port gigabit switch and operate within the MicroBooNE detector network. The Wiener high and low voltage mainframes in their respective racks connect by RJ45 to this switch. The detector control PCs write to databases on a $1 \mathrm{U}$ drive on each of the PCs in this same rack. A fourth $1 \mathrm{U}$ PC functions as the master EPICS server with its own disk and resident database. A sliding 1U $\mathrm{KVM}$ switch/monitor/keyboard available in this rack provides the ability to watch any of the four slow monitoring/control PCs. Another crate in this rack contains a $4 \mathrm{U}$ National Instruments controller and all the necessary electronics to communicate with the gauges, interlock signals, on-detector FBEs, and related equipment.

The remote Intelligent Platform Management Interface (IPMI) also allows for access to all DAQ nodes from outside the detector hall once early commissioning work at KVM stations complete. The KVM system remains for redundant and failover access. Figure 10.38 shows the full design

The MicroBooNE TDR (2/24/2012-DocDB 1821-v12): Electronics/Data Acquisition (WBS 1.5/1.9) Page 136 
for the network. This design, which benefits from the experience of the Nova experiment, consists of three separate networks: the IPMI subnet for booting and monitoring of individual nodes, the internal private DAQ subnet, and the wider Fermilab network used to push out data to Enstore and for mounting libraries and executables to a single location for all nodes. This configuration minimizes build admininistration.

\subsubsection{Monitoring/Control Stations}

Run control takes place through dual-core Linux PC towers located at a suitably large shift station in the detector hall and, separately, in a Wilson Hall control room. Each run control station requires a 4-head video driver and $\sim 4$ GB of memory. Both run stations reside on the MicroBooNE general network. Each can access uBNPC, mainly through web browsers that perform run monitoring.

During commissioning run control will likely take place mainly in the detector hall, with routine shift-based operations moving to Wilson Hall as operations become stable. Run control occurs on the two primary DAQ computers uBDPC and uBNPC, into which the two run stations and other selected MicroBooNE-network machines and users receive access. Remote monitoring of MicroBooNE, but not control, can be given over to off-site clients as the experiment matures to allow remote collaborator shift taking.

${ }^{1}$ David Buchholz and Robert Tilden, "Slow Monitor", MINERvA internal report, online at http://wwwppd.fnal.gov/EEDOffice-w/Projects/CMS/Minerva/Elect/SlowMonitor.pdf (browsed 2012/01/06). 


\section{System Installation (WBS 1.7 and WBS 1.10)}

\subsection{Introduction}

MicroBooNE defines system installation as the process through which elements of the MicroBooNE detector will be received at Fermilab from sub-system fabrication, assembled into the MicroBooNE cryostat at the Fermilab D0 Assembly Building (DAB), and installed at the MicroBooNE detector site in the Liquid Argon Detector Test Facility (LArTF). These tasks, organized under WBS 1.7 and 1.10, include preparation of an assembly area at DAB and of the detector area at LArTF, transportation of detector elements to DAB and LArTF, rigging and other operations affecting the cryostat as a whole at both DAB and LArTF, and coordination of sub-system installations activities. The detailed assembly of the cryogenics (WBS 1.2), TPC (WBS 1.4), PMT (WBS 1.8), electronics (WBS 1.5), and DAQ (WBS 1.9) sub-systems becomes the shared responsibility of the sub-system teams described elsewhere in this TDR and the WBS 1.7 installation team. The installation team (WBS 1.7 and 1.10) serves as the primary interface agent between the sub-systems and resolves all schedule and resource conflicts during the installation.

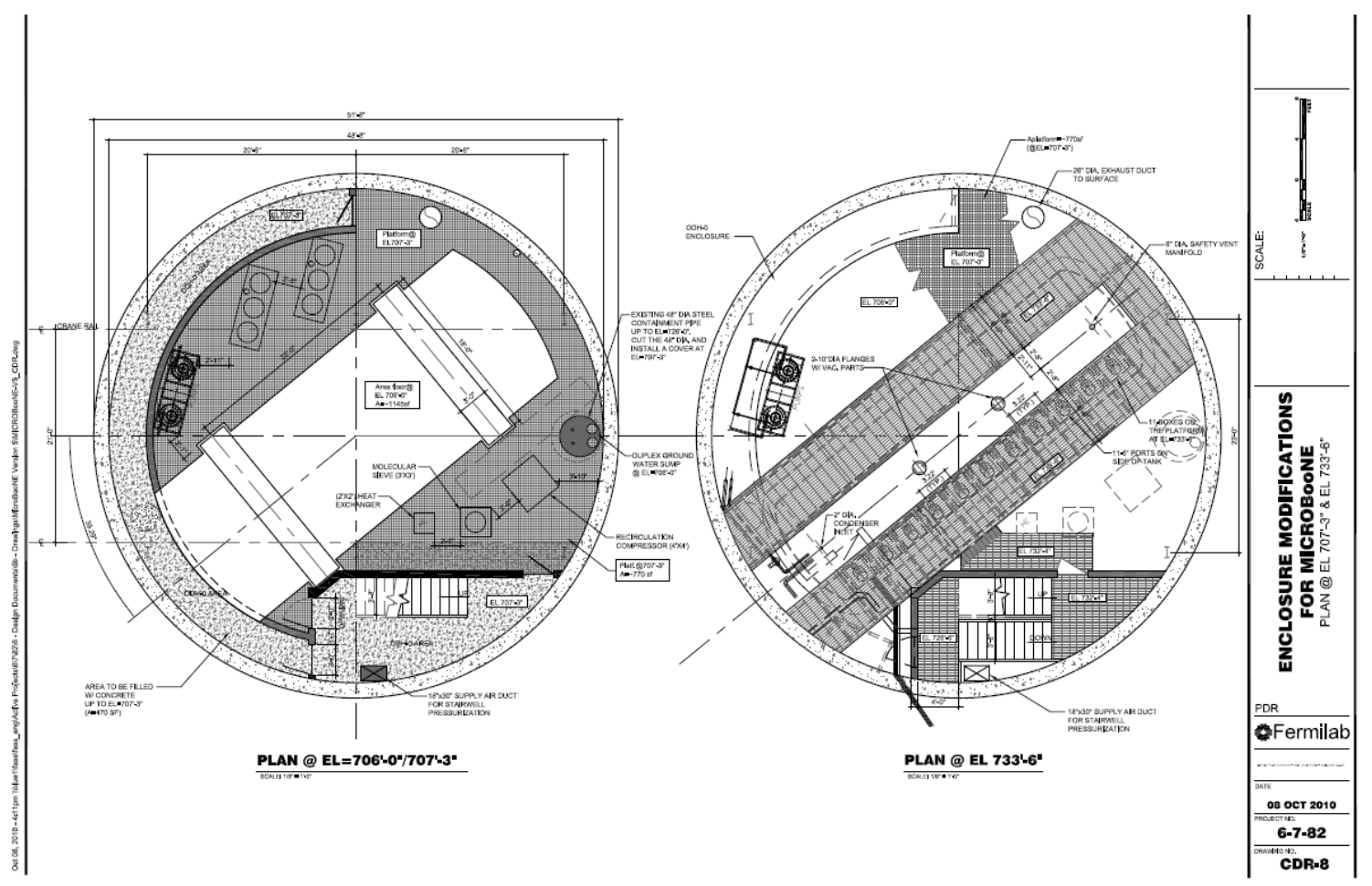

Figure 11.1 Layout of completed MicroBooNE assemblies. 
The overall assembly process splits into three phases: (1) Pre-installation, defined as the preparation of assembly sites, the receiving of all detector elements for MicroBooNE, and the assembly of these elements into a finished detector within the MicroBooNE cryostat vessel ready for transportation to the detector hall; (2) Installation proper, defined as the process of moving the instrumented cryostat to the experimental hall and placing it in the building, and in receiving and assembling the detector hall components that will make the detector functional; and (3) Precommissioning, which consists of bringing all detector systems on line to a state in which the cryostat achieves readiness for filling with liquid argon and subsequent data taking.

Extensive documentation exists in the form of bases of estimates (BoE) for each installation task that produce cost estimates to complete each part of the task. The BoE also describe risk management for cost and schedule, and the development potential contingencies.

\subsection{Pre-installation and assembly site activities}

MicroBooNE plans to assemble the detectors and insert them into the vessel at the D0 assembly building (DAB) at Fermilab, which currently serves as a storage and work space for the D0 experiment. Materials now present in DAB were relocated to create work space for the MicroBooNE assembly area. The Fermilab Particle Physics Division (PPD) provided the resources to clean MicroBooNE's DAB area.

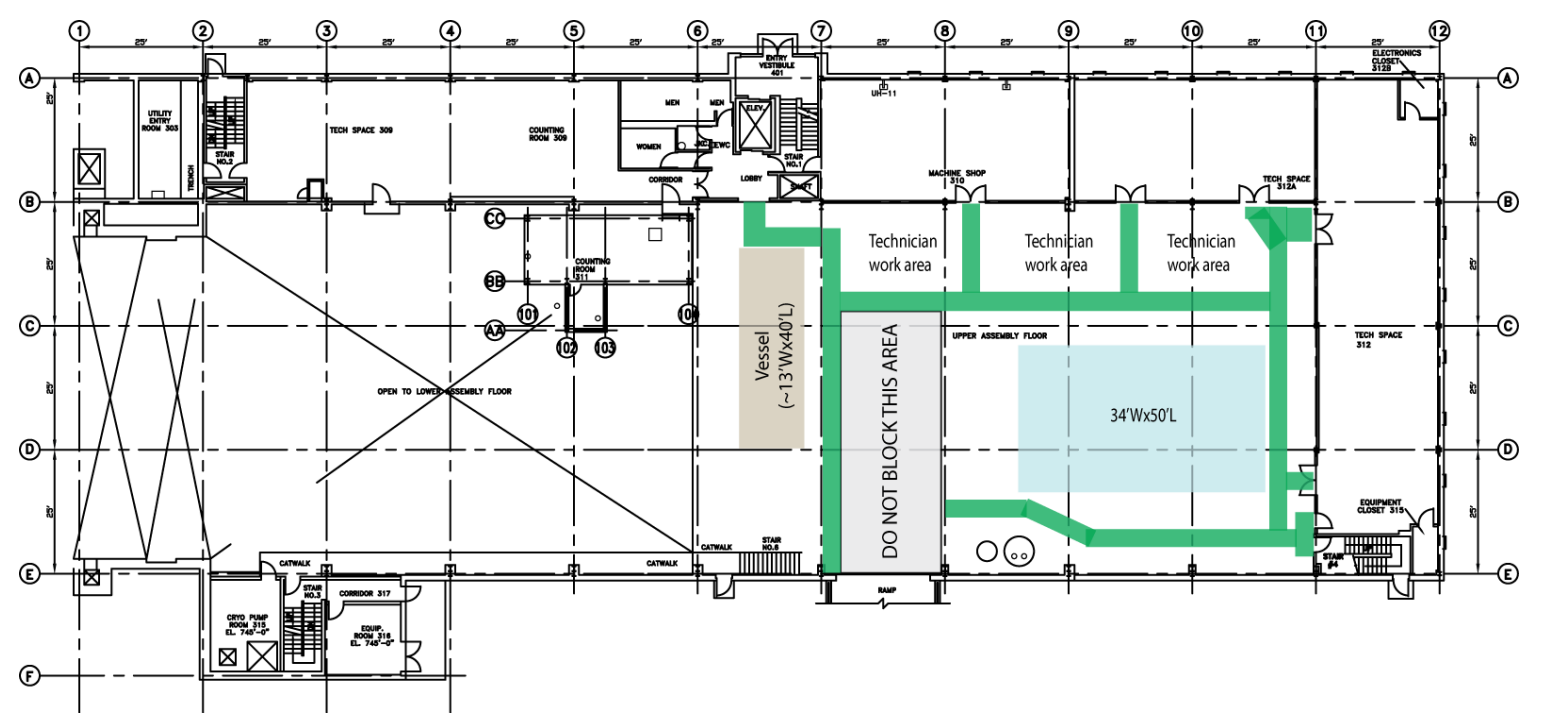

Figure 11.2 Do high bay floor plan. The area from 7-B to 11-E is the region available for MicroBooNE assembly. The colored boxes (thick shaded lines located lower right) represent the vessel and assembly tent, shown to scale..

Figure 11.2 ${ }^{1}$ shows the D0 high bay floor plan. The grid points 7-B and 11-E in Figure 11.2 demarcate the area available for MicroBooNE assembly activities; however, the space bounded by grid points 7-B to 8-E cannot be blocked for extended periods of time because it serves as the truck ramp access area for deliveries.

The tent structure will be assembled in DAB using industrial pallet racks to define the walls and 
engineered lumber (I-joists) to support transparent plastic ceiling panels, shown in Figure 11.3. The tent walls will be lined with fire retardant plastic sheeting to seal off the area for ventilation by HEPA filter fans. This tent will serve as the clean room for assembly activities. The internal working area will be 19 feet tall $\times 26$ feet wide $\times 50$ feet long, although the entire tent footprint is slightly wider because of the 4-foot depth of the pallet racks. The end of the tent near the high bay delivery area (marked "Do not block this area" in the figure) is equipped with a vinyl strip door that will allow us to easily bring large parts in and out. This end will also be temporarily secured to the open end of the MicroBooNE cryostat during installation of the TPC and PMTs. Normal entry to the tent will be through a door attached to one bay of the pallet racks, in the bottom right corner of the tent in Figure 11.2, and shown in the far right of Figure 11.3. There will also be a small gowning area at this entry (not shown). The "clean" area will depend on the building HVAC system for conditioned air, which can be further conditioned with a separate dehumidification unit (already in hand) if necessary. Positive pressure will be supplied by three HEPA filter fans (also in hand). The transparent ceiling panels will provide illumination inside the tent; it will be augmented by stand-alone halogen lights if necessary. A portable power distribution center, already on hand, will supply all electrical needs.

The cryostat will be temporarily stored outside of the tents during the TPC assembly. Its open end will be protected from dust and other contaminants in the DAB by sealing it with plastic. The TPC and PMT detector components will be pre-assembled inside the clean room tent. Upon completion of certification all pre-assembled detector units, the cryostat will be repositioned with its open end facing into the clean room tent to allow the detectors to be installed into the vessel without introducing contaminants. 


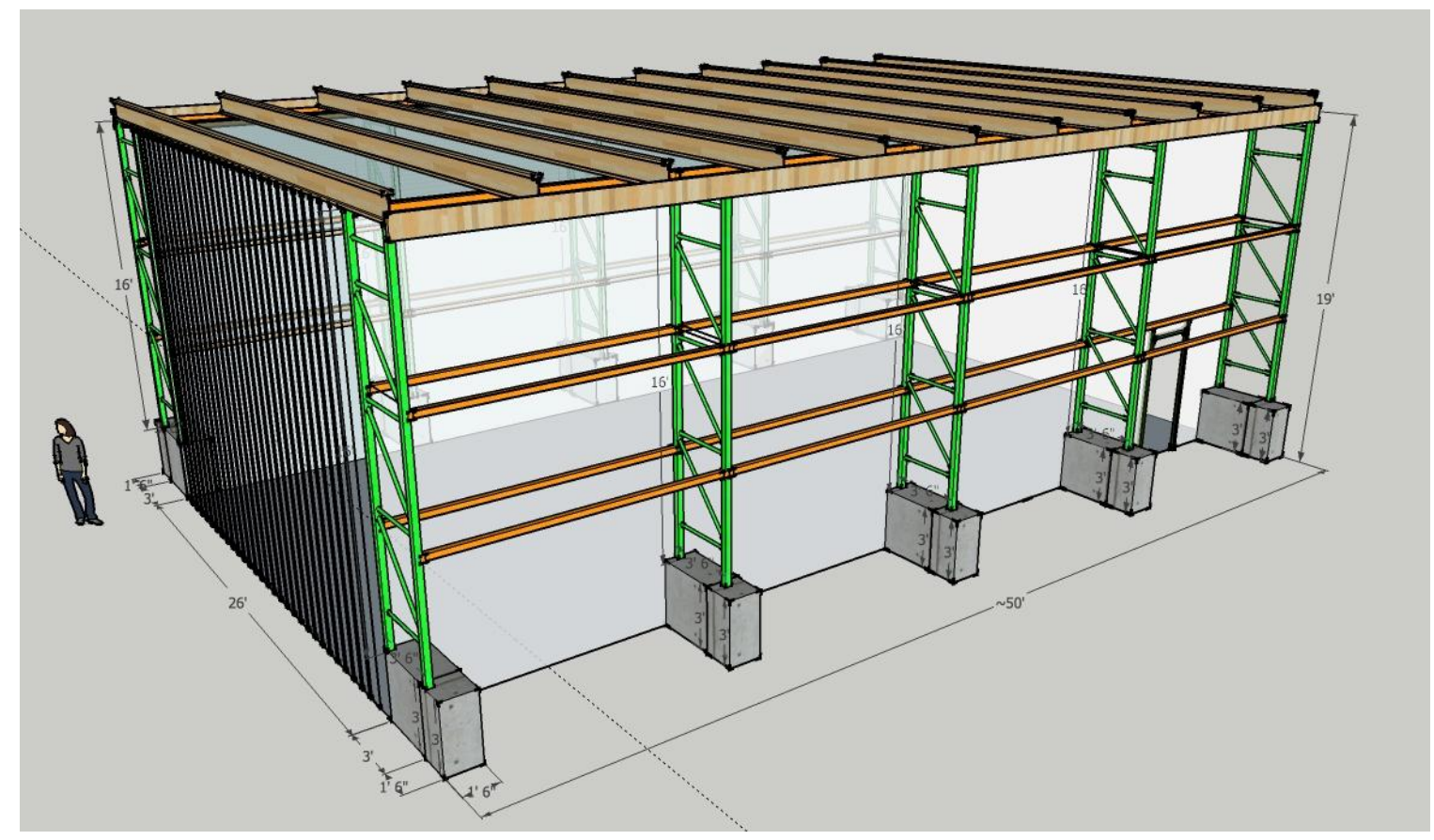

Figure 11.3 Conceptual design of MicroBooNE assembly tent. The industrial pallet racks are bolted to standard E\&F shielding blocks to achieve the required 19-foot height of the tent. Ceiling joists are engineered lumber which span the space between the two rows of racks, and which support the transparent plastic panels that make up the roof. The walls of the tent are lined with fire retardant plastic, and the tent front is equipped with a vinyl strip door..

\subsection{Vessel Preparation}

MicroBooNE will receive the cryostat from the vendor delivered to DAB on the vendor's truck. The truck will be escorted to the D0 assembly building from Fermilab Shipping and Receiving and will be moved into DAB through the 18 foot tall roll-up door on the south side of the building. The vessel will be unloaded by a Fermilab rigging crew to its pre-prepared storage area using the existing DAB 50-ton crane. The MicroBooNE cryostat team will inspect and accept the vessel following procedures defined under WBS 1.3 and described in Chapter 7 of this TDR. A separate inspection may be performed prior to shipping. Fermilab technicians will remove the cryostat end cap and store it in a secure manner.

The D0 crane is a combination 10-ton and 50-ton; the 50-ton crane has a maximum pick height of 21 feet from the floor. We may use a spreader bar to reduce sling length, as shown in Figure 11.4. The cryostat vessel will have engineered pick points. 


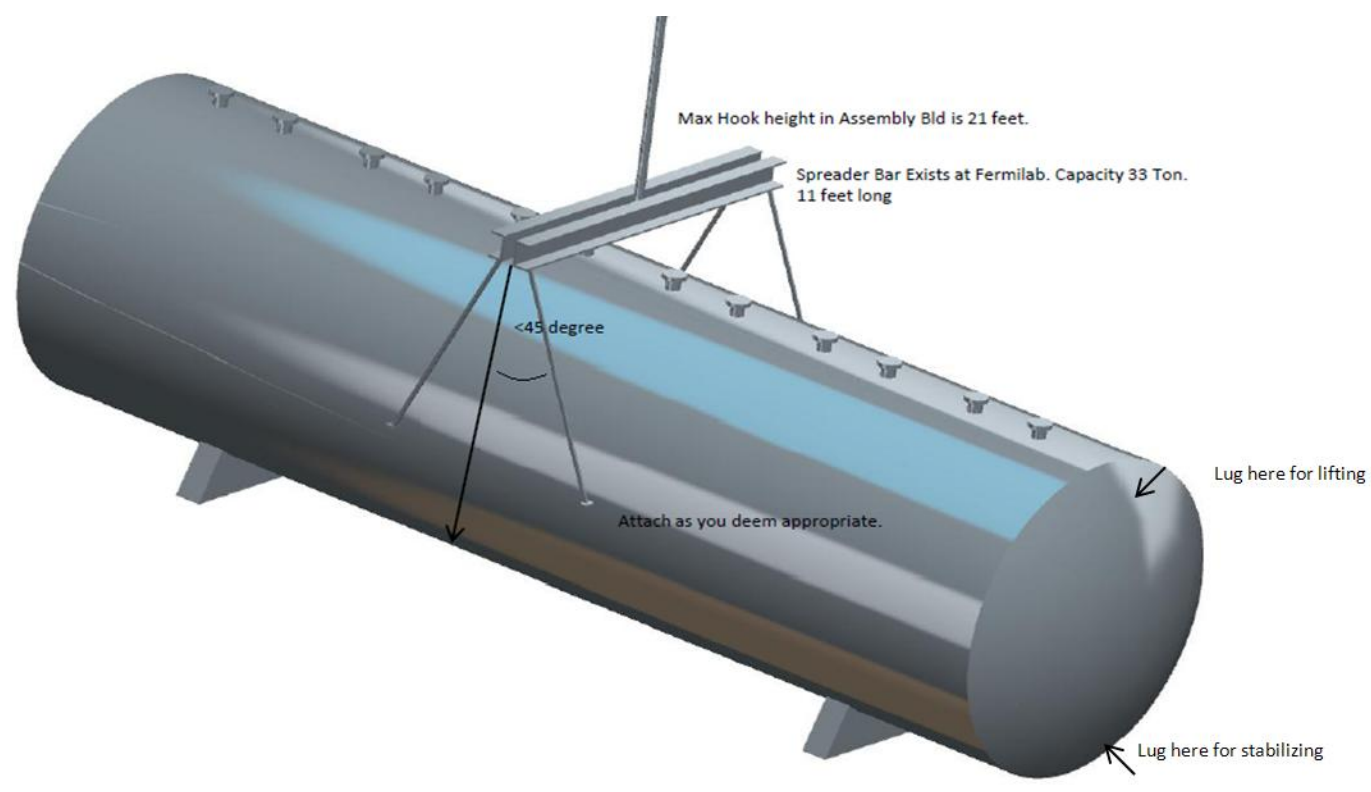

Figure 11.4: Partial specifications for vessel's engineered pick points.

The $18 \mathrm{ft}$ tall by 19 feet wide DAB doorway (Fermilab door 311) features a ramp slope designed with a vertical curve to keep lowboy trailers from hanging up at the top edge at an elevation of 744.50 feet followed by a $6 \%$ slope to the finished floor at an elevation of 737.00 feet. Figure

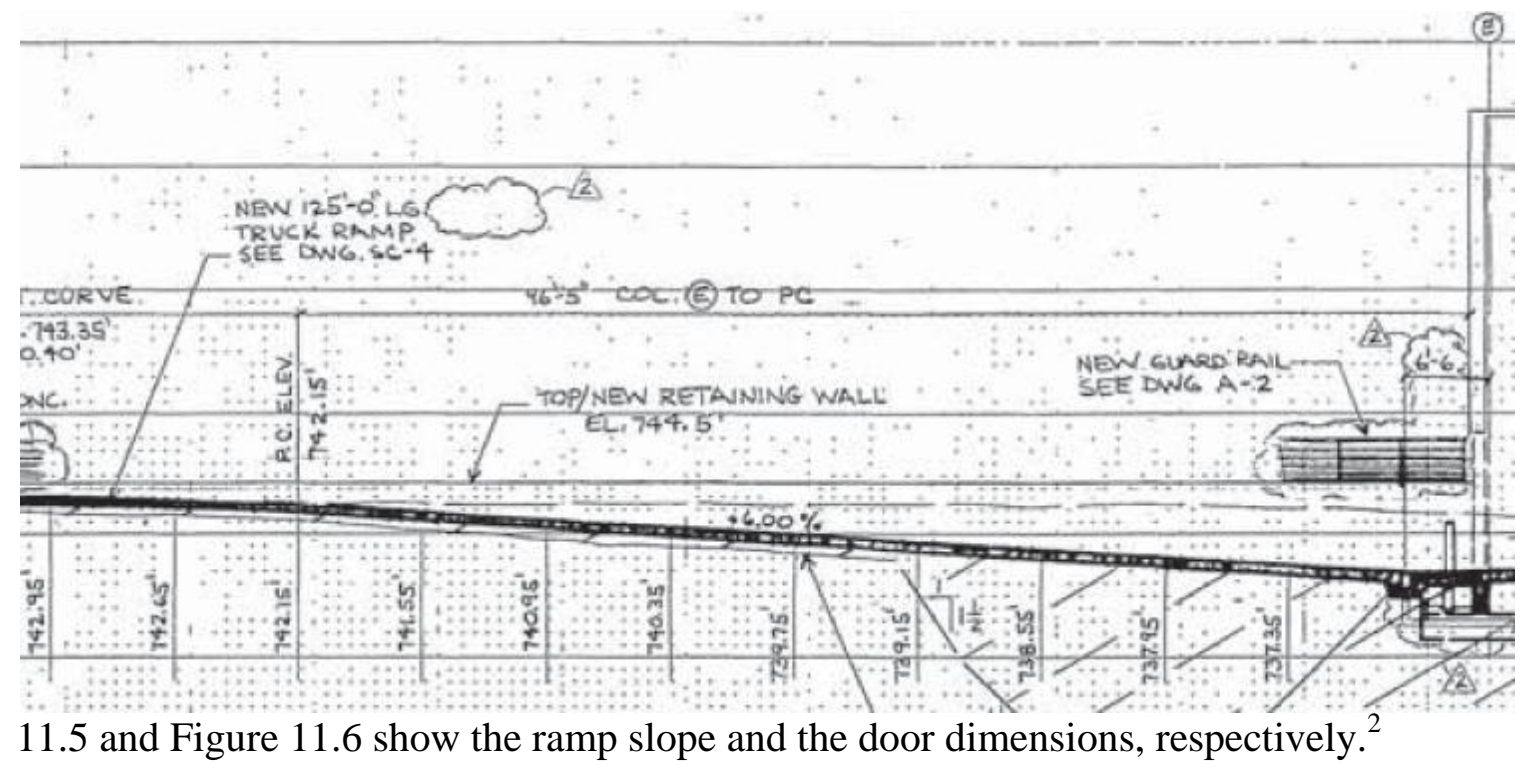

Figure 11.5 Ramp dimensions and grade at south side door of DAB. 


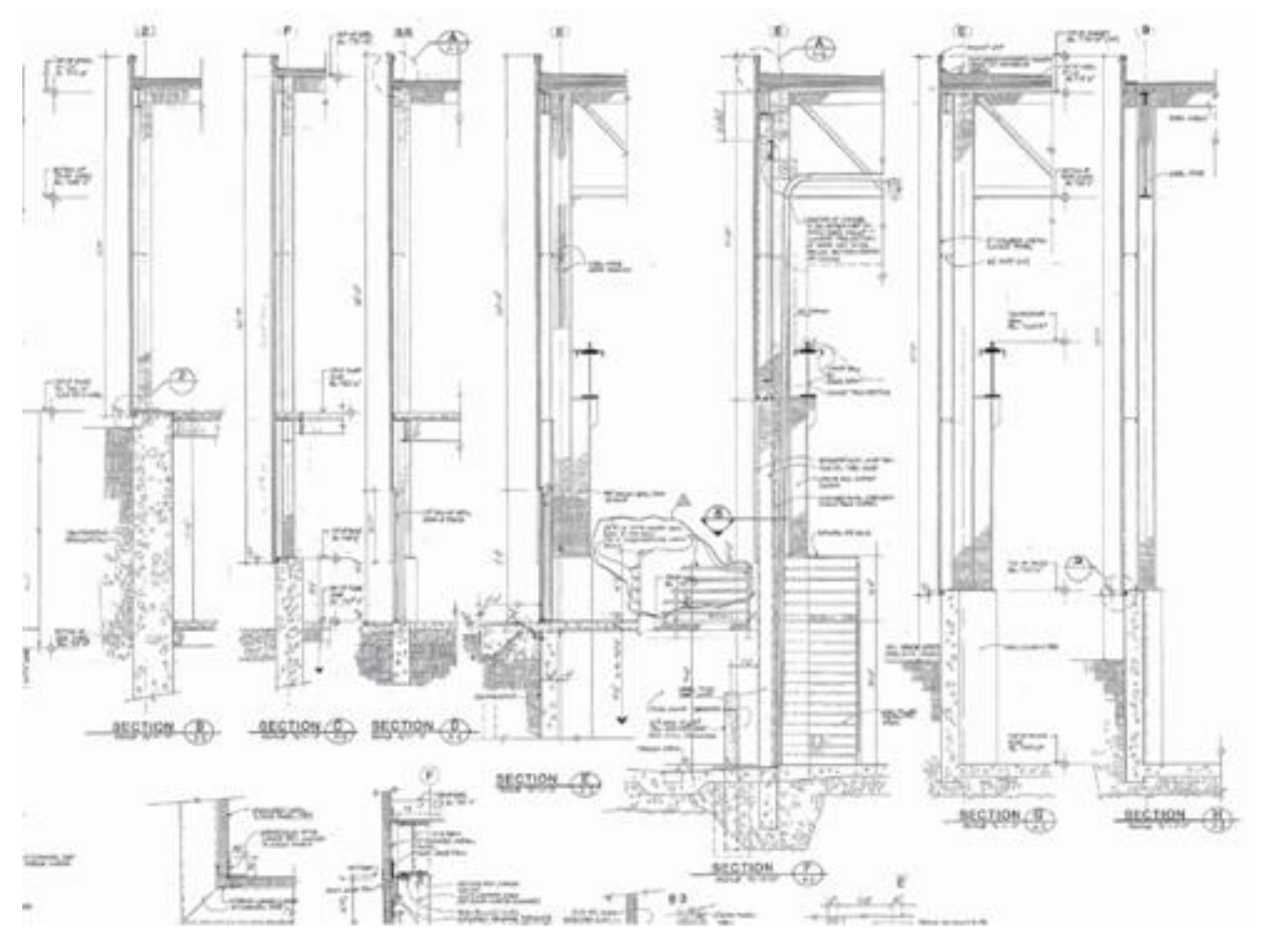

Figure 11.6 Dimensions of south-side door of DAB.

Fermilab PPD mechanical technicians will unload and position the vessel in the appropriate position to allow further work to be accomplished. The cryostat end cap's temporary connections will be disengaged, and the end cap will be removed and set aside. The MicroBooNE cryostat team (WBS 1.3) and the Fermilab alignment group will perform quality assurance and "as found" inspections and measurements.

A professional cleaning company will clean the vessel following the procedures successfully employed on the Fermilab Liquid Argon Purity Detector (LAPD) vessel. A gowning area will be established in the second, smaller tent, and access control rules will be implemented for personnel engaged in clean room activities. The clean room procedures will be adapted from practices established at the Fermilab Silicon Detector Facility (SiDet).

\subsection{Detector Pre-Installation}

The TPC (WBS 1.4) and PMT (WBS 1.8) groups will take necessary and sufficient measures to ensure proper assembly, installation, and functionality of sub-system components. Quality assurance measures will be taken throughout the entire process of receiving, assembling, and installing to guarantee a useful system. 
Survey and alignment will be performed for each sub-system after installation, and the placement of each detector relative to reference points on the exterior of the vessel will be determined. These points will be referenced to an additional set of fiducial marks in the LArTF enclosure after the vessel is moved since the marks used during sub-system pre-installation will be covered with insulating foam.

After all quality assurances have been signed off by the relevant WBS level 2 managers, the cryostat end cap will be positioned onto the vessel and attached. A dry gas purge will be used to protect the detectors until the vendor can weld or bolt the end cap permanently. The clean room tents will be removed. If MicroBooNE adopts the welding option, a code certified contract welder will execute this task, perform the vessel pressure test, and administer the ASME 30.1 stamp of approval.

\subsubsection{Time Projection Chamber}

The parts for the TPC will be prepared, machined, cleaned, and readied for assembly at Yale, Syracuse, and Brookhaven. All parts will be quality inspected and approved for shipment by the BNL TPC crew. Upon arrival at Fermilab, they will become the responsibility of the WBS 1.7 installation team. All TPC parts will be stored in DAB in a clean and secure manner. Any further cleaning of parts will occur in parallel with assembly as needed.

Pre-installation tasks for the TPC will be performed by an installation crew drawn from MicroBooNE under the supervision of the TPC (WBS 1.4) and installation team (WBS 1.7). The WBS 1.7 installation team will be responsible for rigging, mechanical support, alignment and survey support, and supplemental manpower as needed to accomplish the installation. The TPC team will perform all necessary testing and sign off on the installation.

The TPC will be assembled and inspected in the clean tent area outside of the cryostat vessel. Steps in this process include the assembly of the cathode plane and wire frame support structures, installation of the wire plane holder modules, assembly of the field cage structure and top ground plane, survey and establishment of fiducial points, alignment and tensioning of the wire planes, and connection and routing of cables to the feed-through locations.

The finished TPC assembly will be inserted into the vessel with a cart guided by rails that have been pre-installed by the cryostat, as shown in Figure 11.8. Rails outside the vessel will be positioned as an extension of the internal rails. The TPC will be raised with hydraulic cylinders and will be pushed by an electric fork lift until it is in the proper location above the supports. The TPC will then be lowered into its final position and the cart removed. 


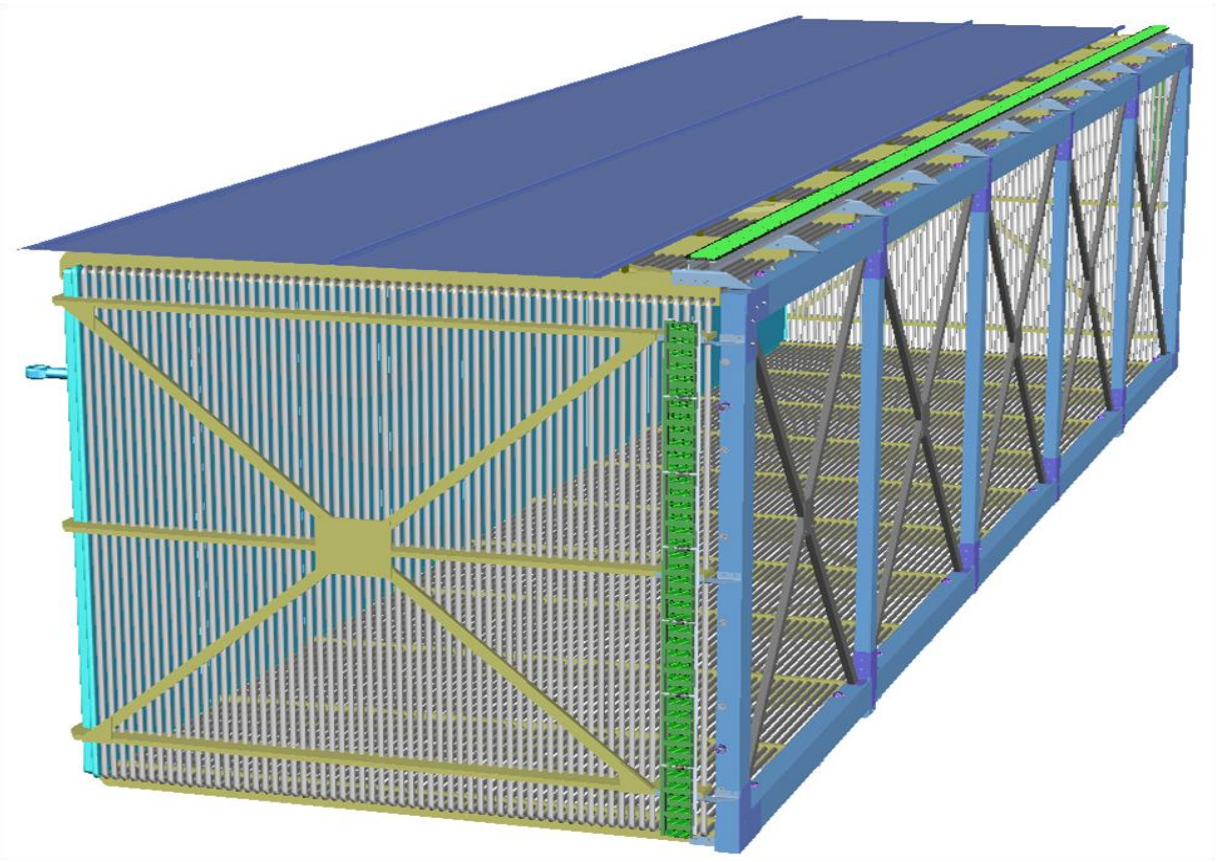

Figure 11.7 Rendition of the finished assembly ready to enter the vessel.

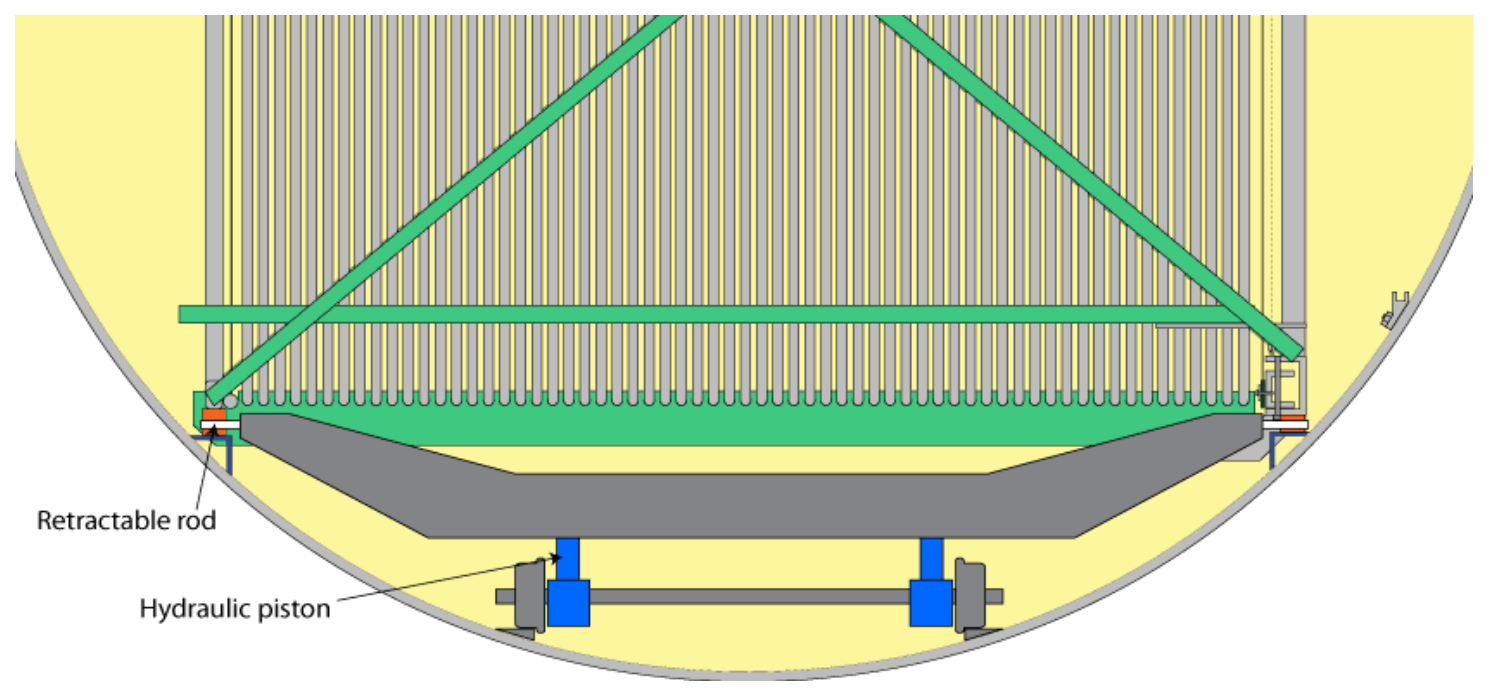

Figure 11.8 Rails for insertion of TPC into vessel.

\subsubsection{PMT Assembly and Installation}

The parts for the PMT support rack will be prepared, machined, cleaned, and readied for assembly at MIT and Princeton. All parts will be quality inspected and approved for shipment before coming to Fermilab. They will be shipped to Fermilab and will become the responsibility 
of the WBS 1.7 team upon arrival. The parts will be received, taken to DAB, and stored in a clean and secure manner.

After the TPC is installed, the WBS 1.8 PMT and WBS 1.7 installation teams will manage the assembly and pre-installation of the PMT system following procedures defined more fully in Chapter 9 of this TDR. Tasks will include assembly of PMT units, attachment of PMT units to the PMT support rack, and connection and dressing of cables for later attachment to the feedthrough. The WBS 1.7 installation team will provide rigging, mechanical support, survey and alignment support, and additional manpower as needed to accomplish the installation. The PMT team will perform all necessary testing and sign off on the installation.

The PMT system will then be installed into the cryostat as shown in Figure 11.9. A similar line of responsibility will be followed in the assembly and insertion of the PMT as with the TPC. An extension of the mounting rails will extend outside the vessel and the assembled PMT rack units will be pushed into the vessel. Other details such as the mounting wedge assembly can be seen in Figure 11.10.

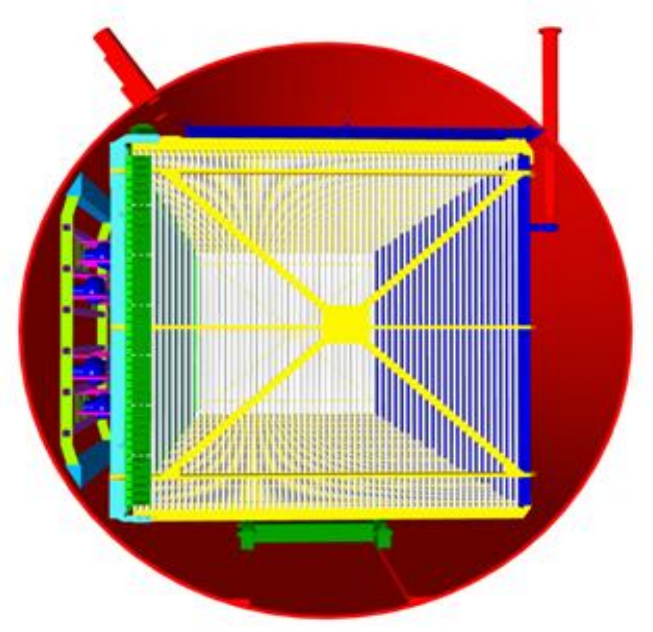

Figure 11.9 The PMT assembly installed in vessel (at left in figure). 
Figure 11.10 Details of mounting wedge and bearings.

\subsection{Installation in Experimental Hall}

At the LArTF experimental hall, the WBS 1.7 team will supervise the installation and alignment of the cryostat support saddles and will arrange for a mobile crane with rigging crew to remove the concrete beams and decking from the LArTF enclosure in preparation for arrival of the vessel.

A professional off-site rigging crew will be contracted to bring the appropriate truck and trailer to $\mathrm{DAB}$, load the MicroBooNE detector onto the trailer using the DAB 50-ton crane, and drive the detector to the LArTF experimental site. The WBS 1.7 team will specify the slow controlled motion procedures for the truck and will arrange for security escorts across the Fermilab site. To insert the detector into the enclosure, the rigging crew will use a crane of sufficient capacity with its pin no closer than 25 feet to the wall of the experiment enclosure.

Roof panels of the MicroBooNE detector building will then be replaced, and a temporary membrane will be installed to prevent water seepage. The detector will then be ready for the application of thermal insulation. 
After the detector is insulated, the catwalks and electronic platforms will be installed. Mechanical technicians will use the in-house crane to reassemble the platforms around the vessel.

From this point the remaining systems will be installed in concert with each other.

\subsubsection{Vessel Insulation}

The detector area will first be cleared of other equipment. The ODH ventilation system will be made functional to provide adequate ventilation, and additional exhaust fans will be added as necessary.

Sixteen inches of foam insulation will be installed in two inch layers. Fiberglass mesh will be wrapped onto the vessel before and after the first layer of foam insulation to provide more structural stability for the insulation. Foster 30-90 Vapor-Safe Mastic will be applied in two layers over the foam insulation to act as a moisture barrier. The LAPD tank is covered with this product, and an example of installation on another tank can be seen in Figure 11.11. A contract insulation specialist will apply the insulation the detector. Fermilab mechanical technicians will assist in the set up and removal of equipment for the execution of this task.

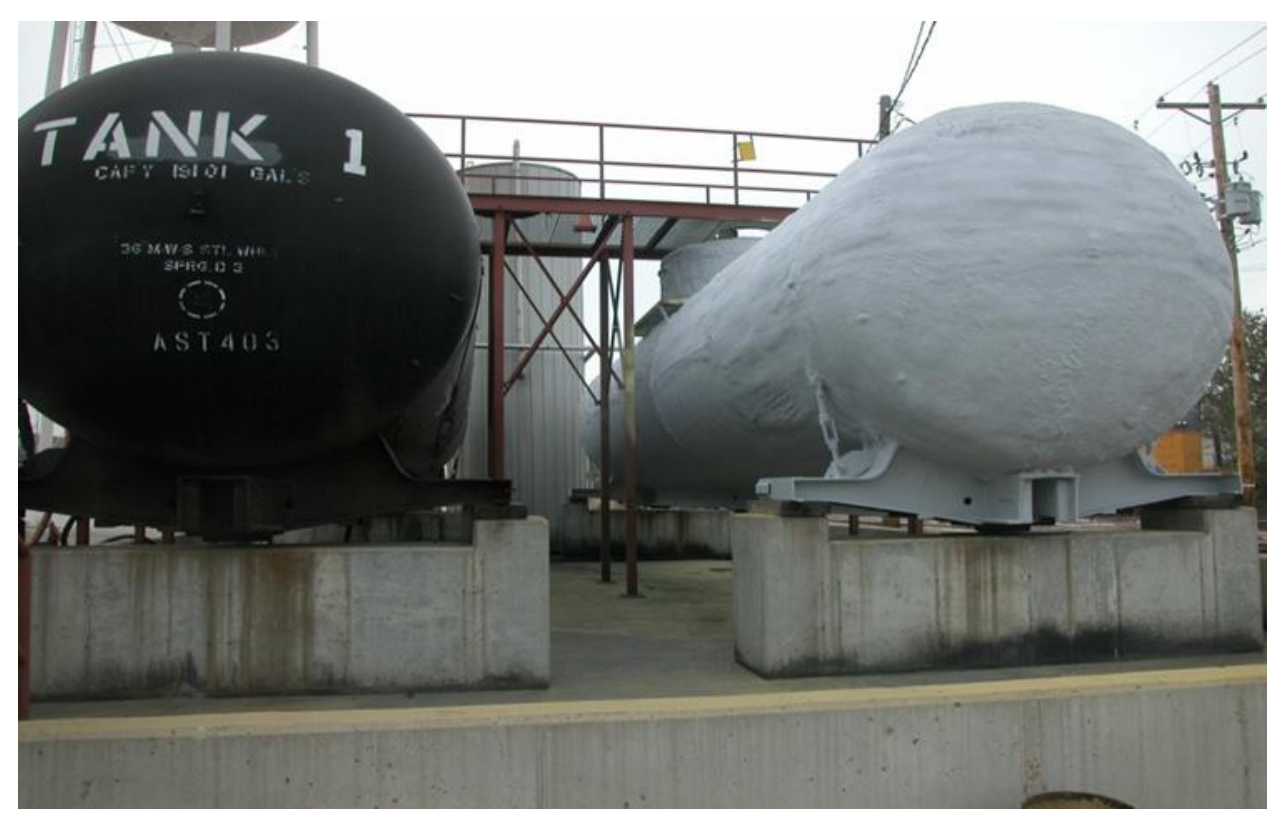

Figure 11.11 Vapor-safe mastic covered insulation on right-hand vessel.

\subsubsection{Electronics Installation}

Relay racks that have been filled and made operational at DAB will be brought to LArTF and installed on the catwalks and in the DAQ room under the oversight of the system experts from the electronics (WBS 1.5) and DAQ (WBS 1.9) teams, and of the WBS 1.7 installation team. 
Cables will be attached to the vessel and cable trays will be mounted for their runs from the vessel to racks and from the racks to the DAQ room. A rack layout already exists, as shown in Figure 11.12. Additional details of this installation may be found in MicroBooNE BoE 1321.

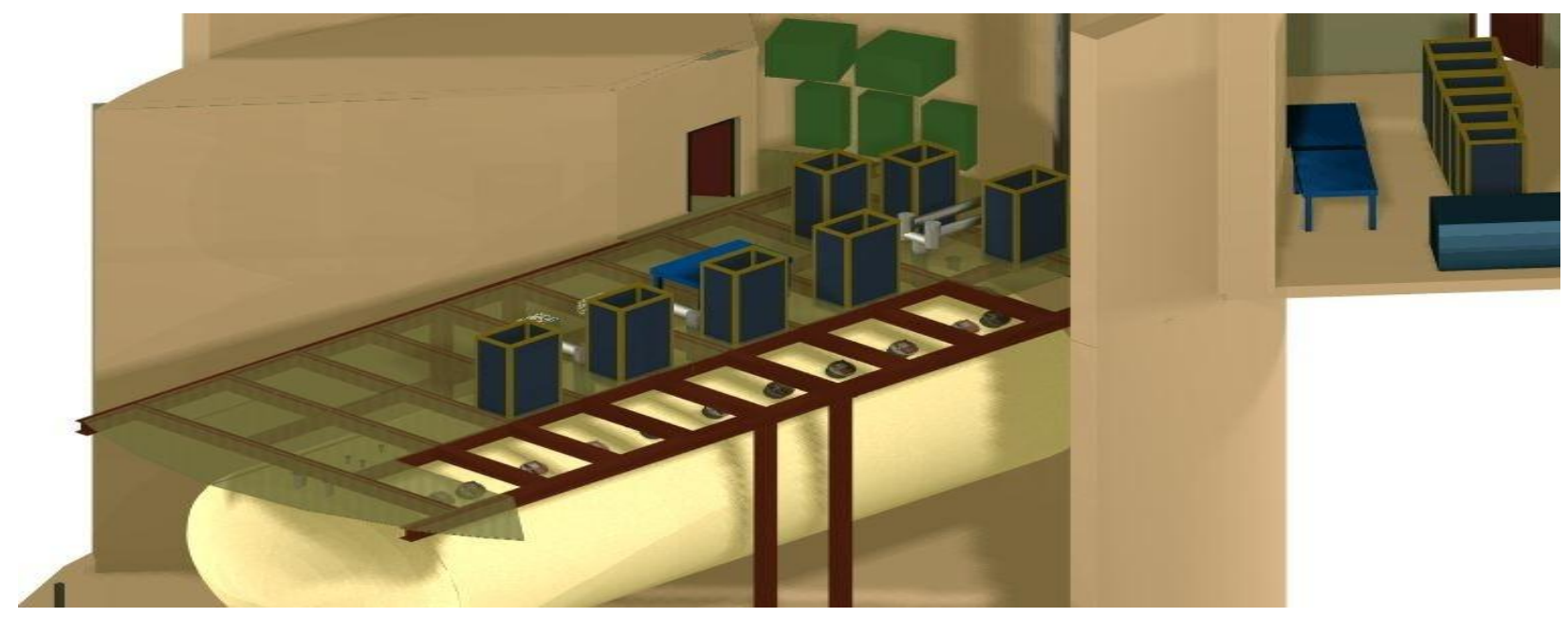

Figure 11.12 Electronics rack layout for MicroBooNE on platform above the cryostat and in the data acquisition room. Three TPC front end electronics racks, a TPC bias power supply rack, and a PMT/trigger rack comprise the row of five racks near the cryostat feedthroughs. The two racks behind these serve the drift field power supply and the cryostat purity monitor. Four of the racks in the data acquisition room hold DAQ computers. One holds communications equipment, and the final contains accelerator division modules. 


\subsubsection{Cryogenic System Installation}

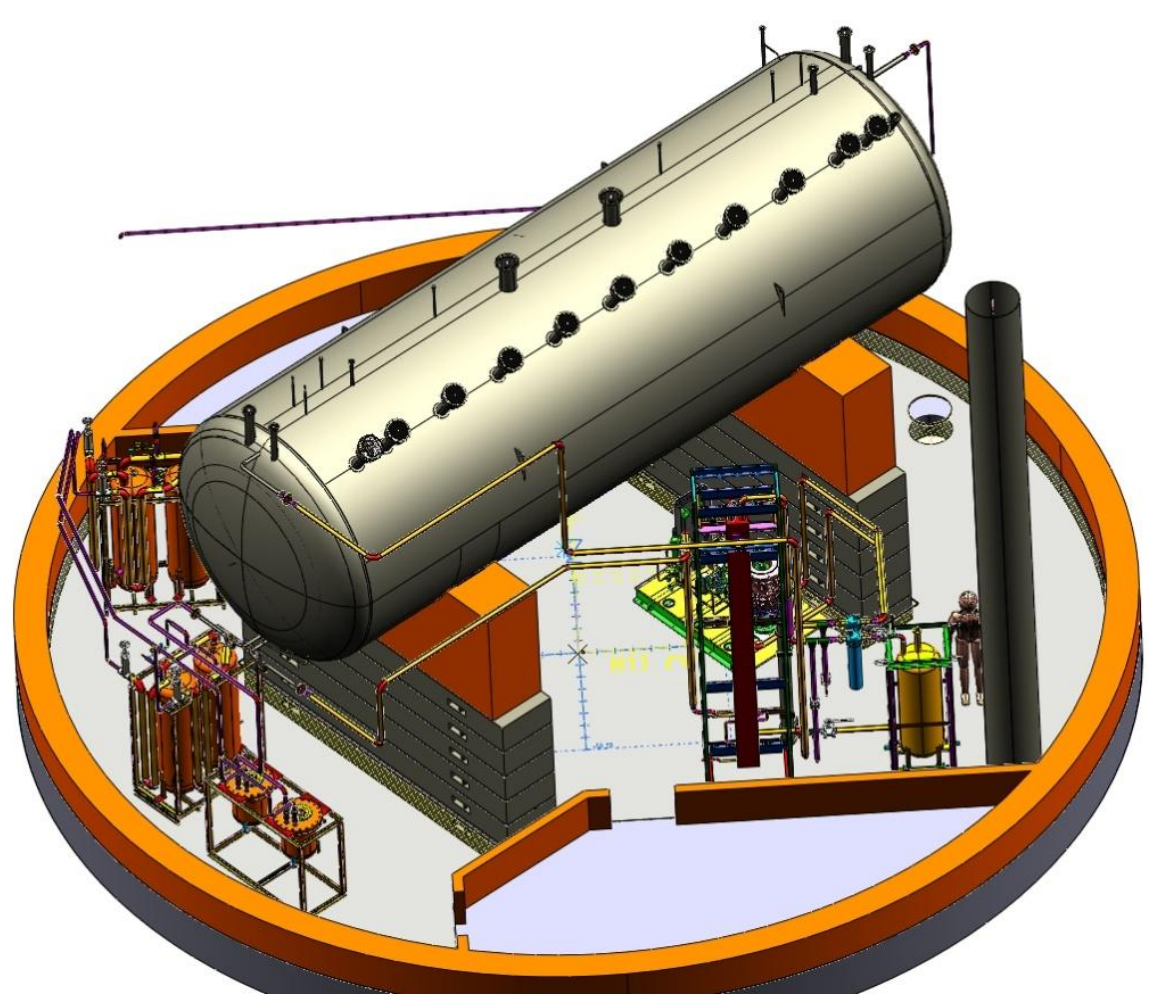

Figure 11.13 Three dimensional layout of cryogenic equipment on the lower level of the LArTF. The two filter skids and the cryogenic pumps are on the left. The cylindrical tank on the right is the molecular sieve filter. The tall chimney-like cylinder provides a 30" pipe for venting argon in an ODH condition. The heat exchange and compressor for cool down are under the cryostat.

This process will entail the positioning and connection of the components of the cryogenic system, which includes cryogenic pumps, molecular sieve filters, purification beds, condensers, purity monitors, transfer lines, relief valves, and other components. Detailed three-dimensional models have been developed to aid in the planning of this process. Figure 11.13 provides an example. Additional details of this system are described in Chapter 6.

System components that have been procured and inspected will be brought over to the LArTF enclosure, placed into their respective positions and secured. Leak checks, pressure, and other procedures required for the safety review of the system will be performed. The cryogenic controls rack, containing instrumentation for monitoring the finished system, will be provided by the cryogenics task (WBS 1.2), and will be installed in the MicroBooNE enclosure by the installation group. After the components have been positioned within and outside of the experimental hall, they will be connected to the appropriated cryogenic fluid supply vessels. Quality assurance testing will include final leak checking, insulating piping, cold shocking (as necessary) and pressure testing. Chapter 6 contains a detailed list of the major equipment used in the cryogenic system. 
The cryogenic system contains foam insulated copper piping, foam insulated stainless steel piping, and vacuum insulated transfer line that employ welded connections. All welds will be performed following Fermilab inspection guidelines ${ }^{3}$. A certified welder will be used to perform the welding. All systems will be leak checked and be installed under the guidance of a cryogenic engineer.

\subsubsection{Pre-Commissioning}

The appropriate system experts along with technician help will inspect and test the data acquisition system and the cryogenic system to ensure the controls are operational and function as desired. Upon completion of this effort the experiment will be ready for cool down and datataking.

${ }^{1}$ 399981.pdf on DocDB entry MicroBooNE-doc-578-v13 with BoE 578.

${ }^{2}$ These are Fermilab FESS drawings D0 C-6.pdf and D0 A-9.pdf.

${ }^{3}$ Weld Insp Guidelines.doc and Weld insp.-1.doc. 


\section{Offline Software}

\subsection{Introduction}

MicroBooNE employs the LArSoft software package for the simulation, reconstruction, and analysis of events collected with the liquid argon TPC (LArTPC). With ArgoNeuT and LBNE, MicroBooNE develops and maintains LArSoft as a general purpose package for LArTPC experiments. LArSoft's design ensures that any LArTPC can make use of its algorithms as long as the particular experiment supplies a properly formatted description of its detector geometry.

\subsection{Framework and Tools}

\subsubsection{Framework}

LArSoft builds on the ART framework designed and maintained by the Fermilab Computing Division (CD) for intensity frontier experiments. Currently, the Mu2e, NOvA, and LArSoft collaborations use this framework. Using $A R T$ means that the $\mathrm{CD}$ supports tools for $\mathrm{I} / \mathrm{O}$, job configuration, and data provenance, freeing LArSoft developers to focus on simulation, reconstruction, and analysis. An MOU under negotiation with the CD will formalize support of the $A R T$ framework for the lifetime of each experiment using it.

LArSoft developers produce compilable code written in $C++$. ART currently employs Fermilab CD's in-house scripting language fhicl; and Fermilab CD's UPS service manages binary distribution of the approximately twenty external packages needed for LArSoft (ROOT, Geant4, Boost, python, $L H A P D F$, etc.). This distribution scheme integrates well with ART on Fermilab computers operating with the prescribed Fermi Linux 5 operating system. Successful off-site implementations of LArSoft have occurred at institutions that carefully replicate the Fermilab scheme.

\subsubsection{Code Management}

The svn products serves as the code base management system for LArSoft, with underlying framework code available from maintained CD repositories and simulation and reconstruction code stored in the LArSoft svn repository. Use of svn allows for frequent and easy user updates of the LArSoft code along with more carefully controlled changes to the framework. Users generally find $s v n$ to be more intuitive than $c v s$, especially with its branching. Keeping track of releases becomes easier, and simpler tools (svn ls, svn $m v$, etc.) make code management clearer. As with $C++$ and $A R T$, post-docs and students using svn acquire useful skills for modern era software tasks.

\subsubsection{Build System}

LArSoft uses the SLAC-originated build system SRT. LArSoft experience with SRT under the previously used $F M W K$ framework gave confidence, since borne out, that $S R T$ would be the quickest system to deploy with $A R T$ to get users up and running quickly. SRT comprises an easyto-use package that builds private dynamic libraries for users against the full public build release. The seamless interoperability of private builds with the public build release makes $S R T$ very attractive to users. A cron job builds a new public release from the head release in the repository each night. 


\subsection{Event Display}

The LArSoft event display gives code developers and analysts an indispensible tool in understanding neutrino events in liquid argon. It features easy startup and management with an fhicl script that allows for both specific event selection and for selective enabling for viewing of raw data and reconstructed objects. The event display has been tested with simulated events and ArgoNeuT data, and the code has been written with ease of generalization to MicroBooNE in mind. Figure 12.1 shows an example event display frames from ArgoNeuT events.

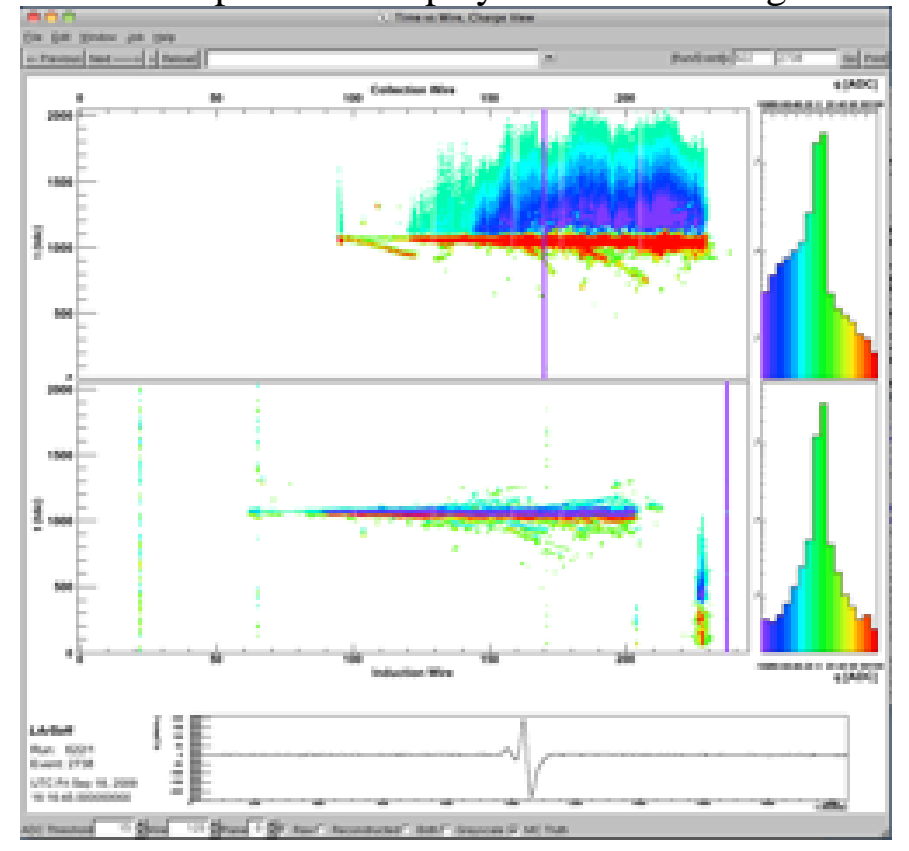

Figure 12.1 An event display image in wire-time space of a candidate electron neutrino CCQE event in the ArgoNeuT data. The top panel is the collection plane view while the middle is the induction plane view. The histograms to the right of these panels show the ADC counts on the wires for each plane. The bottom panel shows the signal on wire 120 of the induction plane as a function of time. The mostly-blue (small signal) shadow at large TDC values in the collection plane is an artifact of the electronics and not-yet-completelytuned digital signal processing.

\subsection{Simulation}

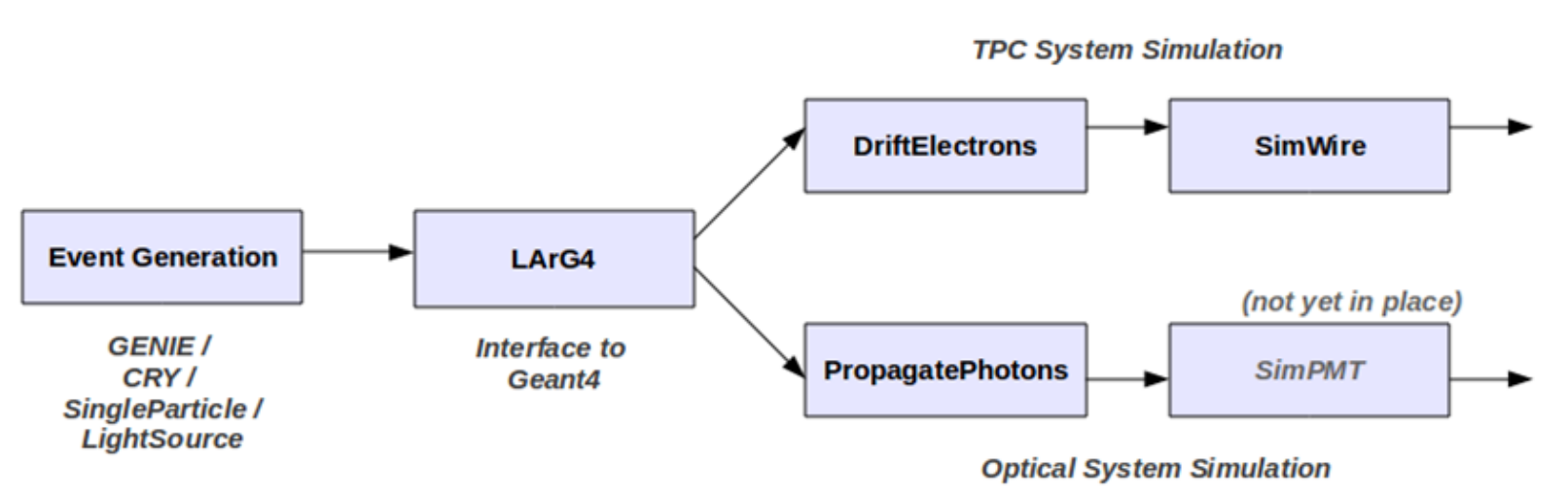

Figure 12.2 Structure of a $L A r S o f t$ simulation job.

Simulation jobs in LArSoft follow a modular structure, with each module reading input data objects from and writing output data objects to the event record. An external event generator 
module defines the objects to be simulated. Geant4, through an interface called LArG4, implements the detector geometry and manages the interaction of primary and secondary particles with the detector media. Custom modules developed for LArSoft simulate the TPC (DriftElectrons, SimWire) and PMT (PropogatePhotons, SimPMT) detector responses. Figure 12.2 depicts the structure of a typical simulation job.

The LArSoft simulation interfaces with standard external packages. The GENIE 1 event generator models neutrino interactions. The $C R Y^{2}$ program simulates cosmic ray interactions. Geant $4^{3}$ models the detector response.

Event generation, LArG4 simulation, and DriftElectrons modules exist in advanced states of development that have already been used to perform TPC simulations for the ArgoNeuT experiment. A prototype exists for the PMT-system specific PhotonPropagation module. The experiment-specific electronics simulations SimWire and SimPMT remain under development, with approximate place-holder approximations in place.

\subsubsection{Geometry}

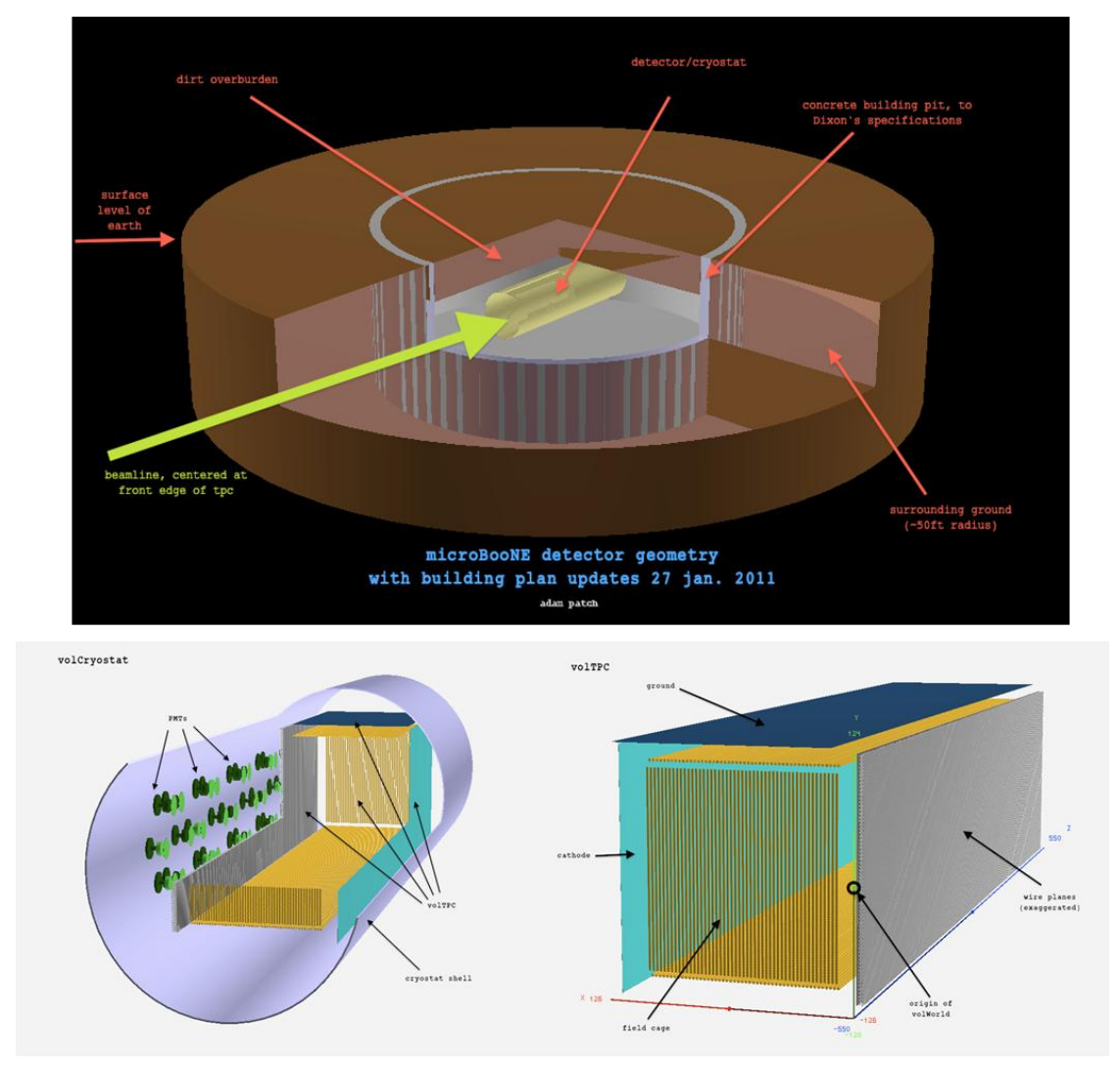

Figure 12.3 Views of Geant4 implementations of the MicroBooNE detector geometry in LArSoft. The top picture shows the entire detector hall region surrounding the cryostat. The lower left picture shows the interior layout of the active detectors (TPC+PMTs) in the cryostat, and the lower right picture shows details of the TPC.

Geometries in LArSoft defined in the GDML4 language currently exist for MicroBooNE, 
ArgoNeuT, and several proposed LBNE liquid argon detectors. With a description supplied via a text file, GDML defines a nested hierarchy of volumes and descriptions of contributing materials. A series of scripts create the MicroBooNE detector geometry. These define the size and shape of the world, cryostat, and TPC volumes and perform the placement of repeated elements such as TPC wires and PMT assemblies. Some steps in the event generation and detector simulation act on specifically named volumes and associate particular properties with them; these will be described in relevant sections that follow. Figure 12.3 shows several views of the LArSoft implementation of the MicroBooNE geometry.

\subsubsection{Event Generation}

An event generator starts the standard simulation chain, producing a set of Monte Carlo "truth" particles that are then propagated through the LArG4 detector simulation. The EventGenerator package in LArSoft contains interfaces to several event generation modules, some of which are internal to LArSoft, and others which are external packages.

Beam neutrino interactions can be simulated using GENIE, which takes as input a flux description to be sampled from a specified flux file, the volume over which interactions are generated specified by its name in the GDML geometry description, and other parameters passed through a script, such as neutrino flavors, beam properties, and target information.

Cosmic ray interaction simulation occurs via the CRY5 package, which allows specification of detector latitude and altitude, cosmic ray interaction energy threshold, and the cosmic ray interaction volume centered at the origin of the TPC volume.

For validation and reconstruction studies, LArSoft additionally incorporates a particle gun event generator called SingleParticle. This allows the user to specify a number of particles to generate by their PDG particle code and desired kinematics. Kinematics can either be specified exactly or sampled from a Gaussian or uniform probability distribution.

For studies of the optical systems and construction of the fast optical simulation library, LArSoft incorporates a LightSource event generator, which simulates an isotropic light source of a specified size, shape and intensity. The light source can be either static, or moved through the detector on an event by event basis. For a moving light source, positions and properties are defined either systematically by dividing a volume into rectangular voxels and stepping through them one by one, or via a set of light source descriptions supplied via a text file. The event generator writes out a description of the light source for each event for use in further analysis or library building.

\subsubsection{The LArG4 Detector Simulation}

Truth particles generated in the event generator step pass to a Geant46 based detector simulation through the $L A r G 4$ interface. $L A r G 4$ parses the geometry GDML file to create a Geant 4 detector description using the built-in GDML parser that interfaces to LArSoft via the DetectorConstruction LArSoft class.

\subsubsection{Physics List Handling}

The user can specify which physics processes to enable using LarG4's configurable physics list 
system, which allows the specification of enabled physics constructors on a job-by-job basis. At the most basic level, a physics constructor comprises of a list of Geant4 physics processes and the particles to which these processes apply. Available physics constructors inherit from the G4VPhysicsConstructor class and register themselves with the custom physics table at compile time using a CustomPhysicsFactory class. Default Geant4 physics constructors interface to LArSoft in the CustomPhysicsBuiltIns file, and LArSoft specific physics constructors include a call to the custom physics factory in their implementation file. Using this system, $\operatorname{LArG4}$ becomes extendible with new physics processes that can be enabled or disabled on a job by job basis, without requiring a rebuild as would be standard with a hard coded physics list. The default list of enabled processes corresponds to those enabled by the QGSP_BERT Geant4 physics list.

\subsubsection{Physics List Configuration for Optical Simulations}

Optical physics simulations in LArSoft can take one of two forms, "full" or "fast" simulation, the latter of which involves several simulation modules to be described separately in Section 12.4.5. Full simulation involves stepping every optical photon individually through the detector volume. Typical photon yields for an event can be in the $10^{5}$ range; hence this is a very computationally intensive procedure. The OpticalPhysics physics constructor has been specially adapted for LArSoft and includes scintillation and Cerenkov photon production, Rayleigh scattering, reflections at boundaries, absorption at boundaries and in the bulk, and wavelength shifting physics processes. The following paragraphs describe these configurations more fully. Selected full optical simulation results can also be found in Chapter 9.

The scintillation production configuration uses a photon momentum spectrum of $9.7 \pm 1 \mathrm{eV}$ and a yield of 24,000 photons per $\mathrm{MeV}$ of energy loss, incorporating both a fast and a slow component, which can be scaled by a quenching factor specified for each scintillating particle. These quenching factors, and the possibility of utilizing a more systematic specification of the quenching per particle, require further investigation. Cerenkov photon production utilizes yields and energies corresponding to the standard Frank-Tamm spectrum of Cerenkov radiation.

OpticalPhysics sets the scattering length to $90 \mathrm{~cm}$ and the absorption length to $2000 \mathrm{~m}$ for all wavelengths for the purposes of preliminary studies. Since $128 \mathrm{~nm}$ wavelength scintillation photons comprise the vast majority of photons produced, neglecting the wavelength dependence of these parameters should not cause a significant problem.

A simplified reflectivity model supplies each type of boundary in the detector with a wavelength dependent total reflectivity and a specular/diffuse reflection fraction. Preliminary studies performed to date assume only the steel/argon boundaries at the edge of the cryostat to be reflective, with a total reflectance of $25 \%$ and a $50 \%$ specular/diffuse reflection fraction, and all other surfaces ( wires, field cage, etc) taken to be being opaque.

Although not required in the current sensitive detector scheme, wavelength shifting in the TPB coating can be included as a physics process. The model assumes absorption of a certain fraction of photons incident on the TPB according to a measured TPB absorption spectrum. Reemission then occurs via a Poisson-sampled number of photons emitted with frequencies sampled from the TPB emission spectrum. The Poisson mean for reemission equals $N \times$ the absorbed number, with 
$N=1$ the nominal setting.

\subsubsection{Sensitive Detectors and Output Objects for the TPC Simulation}

The LArG4 simulation outputs for the TPC detector a data object consisting of a three dimensional map of charge deposits from each track over the liquid argon TPC volume, called a LArVoxelList, and then simulates drift of these charges in the electric field towards the wire planes. LArG4 generates the LarVoxelList map by dividing the TPC volume into cube shaped regions ("voxels"), each of which receives a unique ID, and attaching a Geant4 sensitive detector to these regions. During the particle stepping simulation, the module records energy loss by any particle in such a region along with the relevant track ID and voxel ID. In order to improve simulation performance, the voxelization scheme is subsumed within a Geant4 parallel geometry. In this way, only particles that will produce scintillation deposits need to be re-stepped at the voxel boundaries, and other particles, for example, optical photons, can be allowed to take much longer steps in the argon volume.

\subsubsection{Sensitive Detectors and Output Objects for the Full Optical Simulation}

The MicroBooNE geometry includes a detailed description of photomultiplier tube assemblies, as described in Chapter 9. LArG4 outputs the data object PMTHitCollection for both fast and full optical simulations. PMTHitCollection consists of a collection of the 4-positions and 4momenta of each photon that has stepped across the boundary of each PMT sensitive volume. A Geant4 sensitive detector called a PMTSensitiveDetector produces these collections. PMTSensitiveDetector attaches to any volume in the GDML geometry with the appropriate name as supplied in the LArG4 job configuration. A PMTSensitiveDetector stops and kills the track of any optical photon stepping into the relevant volume and stores its kinematics in the relevant PMT hit, indexed by PMT ID. Optical systems also exist within a parallelized geometry so that the PMT assemblies can be made, by default, insensitive to other stepping particles.

Two possible detection schemes can be utilized. A sensitive detector can be attached to the PMT lens, and all wavelength shifting processes in the TPB plate accounted for within the simulation; or the sensitive detector can be attached to the TPB plate itself, and the efficiency of the overall assembly can be accounted for with multiplication by a global efficiency factor. Currently the simulation utilizes the second strategy, with the global assembly efficiency set to 0.03 , as determined by calculation. An upgrade will incorporate the measured efficiency for each assembly determined in a test stand before installation. The method whereby the sensitive detector is attached to the PMT lens itself has the advantage of accounting for the full radial dependence of the efficiency of detection for light striking the TPB coated plate. It has the disadvantage that the absolute conversion efficiency and output spectrum of TPB, as well as the precise TPB deposition over the area of the lens must be known.

\subsubsection{The UserAction interfaces to LArG4}

$L A r G 4$ incorporates a user action management service whereby interfaces to Geant4 can be made at the beginning or end of a run, event, tracking, or particle step. The UserActionManager class provides hooks into Geant4 processing at each of the above interface points, and calls the relevant routines of any registered UserAction class at the appropriate time. The design model requires that one UserAction class should be application specific and that it can include methods that run at one or several of the above hook points. Several UserAction classes already exist for 
technical LArG4 simulation tasks, and others may be developed by future analyzers to further explore the behavior of stepping particles in the simulation.

\subsubsection{Material Properties Handling}

Material properties required by Geant 4 such as Birks constant, liquid argon absorption spectra, reflection spectra at steel / argon interfaces, and several others, cannot be supplied within the $G D M L$ geometry specification. Hence a separate material property loading service exists called MaterialPropertyLoader that reads parameters from a supplied configuration and loops through the parsed geometry after detector construction. When a volume of the relevant material is found, the set of properties read from the configuration for this material are attached. The configuration format for the parameters file may change in future versions of LArSoft.

\subsubsection{Electron Drift and TPC Electronics Simulation}

Once the map of charge across the volume has been simulated using LArG4, the module simulates the drift of this charge to the wire planes and the expected wire response. The DriftElectrons module simulates the charge drift based on properties supplied by the LArParameters service, which supplies the global properties of the liquid argon relevant to TPC operation in LArSoft. The drift velocity calculation uses the argon temperature and applied electric field7, and the charge yield determination per $\mathrm{MeV}$ of energy accounts for reductions via recombination in accordance with Birks law8. Diffusion parameters are supplied directly to the DriftElectrons module.

DriftElectrons divides the charge in each voxel is divided into several parts of equal size, referred to in the drift simulation as "clusters" (not to be confused with reconstruction clusters), which are each drifted individually in the simulation. Any cluster reaching the wire plane produces a simulated Electron object that contains information about the nearest TPC wire, the arrival time, the amount of charge in the cluster, and the voxel of origin.

The collection of Electron objects then serves as input to simulate a TPC wire response. This aspect of the simulation remains under development, but a good working model exists incorporating wire geometry, pulse shaping, electronics noise and ADC sampling and scaling effects.

\subsubsection{Fast Optical Simulation Modules}

Since the stepping of every photon generated in an event rapidly becomes a computationally intensive process, an alternative, fast library sampling simulation is in development. Currently this exists in the prototype state described below.

LArG4 divides the optical fiducial volume into optical voxels, with the scintillation light production intensity and time profile in each voxel recorded in a PhotonVoxelList object, analogous to the LarVoxelList object described in Section 12.4.4. The sensitive detector configuration comes out somewhat different to that applied to the charge voxels, since the isotropic light production results from a very specific physics process. This allows for improve efficiency by not requiring stepping particles to know about the presence of optical voxels, but rather supplying a modified scintillation process, FastOpScintillation. The FastOpScintillation 
process acts like the G4Scintillation process, but rather than producing OpticalPhotons as secondary particles, it fills the PhotonVoxelList with the map of scintillation photon production across the detector volume. The photon voxels differ in definition from charge voxels since they undergo a separate size optimization.

A separate PhotonPropagation module runs after the LArG4 simulation and reads the PhotonVoxelList to sample an expected response from a library of pre-generated PMT responses. The library contains a set of PMTHitCollections giving the expected final 4-positions and 4momenta of detected photons for a very intense light source at each voxel in the detector. By comparing the intensity of the scintillation light source present in each voxel in the event to the intensity of the light sources used to generate the library file and then applying Poisson fluctuations, the number of photons to sample for each PMT in the geometry can be calculated. The library file provides a set of photons to be sampled. PhotonPropagation then smears their detection time by the time profile of scintillation in each voxel and, by combining the expected responses from the light in each voxel, generates a PMTHitCollection representing the expected detector response for the event.

The photon library depends on both geometry and voxelization scheme, and changes to either require a full regeneration, a one-time computationally intensive job. The LightSource event generator supplies the intense sources of $9.7 \mathrm{eV}$ photons for library generation, and the full simulation package steps all optical photons through the geometry. A module called PhotonLibraryBuilder, part of the PhotonPropagation package, stores the PMTHitCollections produced by $L A r G 4$ for light sources placed in each voxel, as well as the light source intensity. Figure 12.4 shows the library building and sampling simulation chain. 


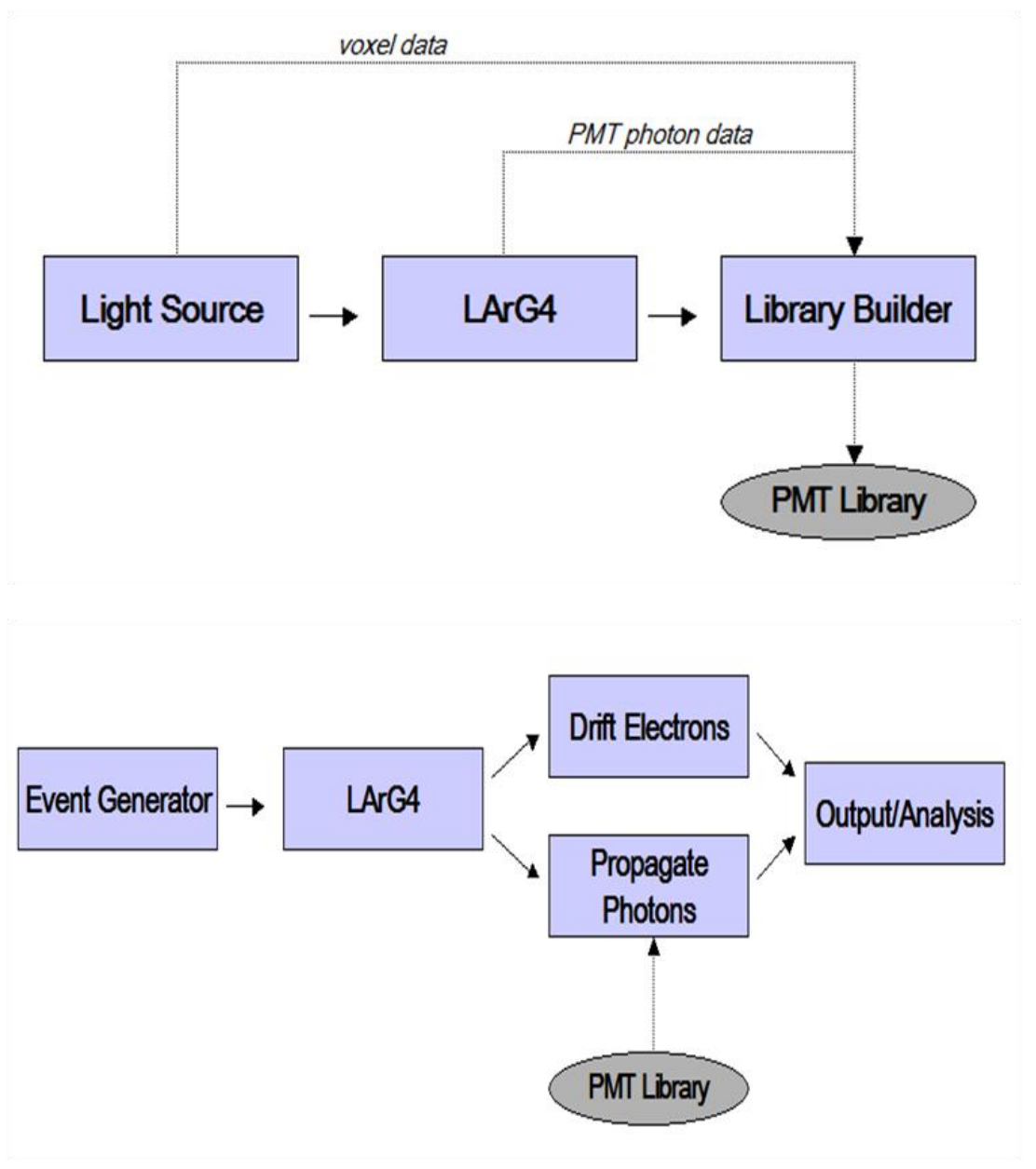

Figure 12.4 The LArSoft simulation chain for voxelized PMT response library building (top), and the LArSoft simulation chain for voxelized PMT response library sampling (bottom).

\subsection{Reconstruction}

The reconstruction chain in $L A r S o f t$ benefits from the experience of multiple experiments. Each LArTPC provides essentially the same basic information after accounting for small detector differences. Thus, algorithms developed for one experiment can be directly used by another, as long as the differences in geometry are properly accounted for in the algorithms. The reconstruction chain proceeds in the following steps: i) First, a calibration converts the raw wire readouts (digits) into calibrated signals on wires. ii) Next, localized wire signals above a tunable threshold define hits. iii) Hits group into clusters. iv) Clusters further group into prongs, which can be either tracks or showers. v) Prongs originating from a common point form vertices. vi) Finally, prongs and vertices build into events.

The analysis then proceeds with reconstructed events. The LArSoft reconstruction chain is complete in that initial algorithms for each step currently exist. More effort remains to demonstrate that every event topology can be handled in an automated way in the reconstruction chain. Detailed studies of various event classes underway result in continued improvement in the understanding of the performance of each reconstruction algorithm. 
The reconstruction algorithms in LArSoft benefit from several advances in image analysis techniques developed over the past decade. For example, the algorithm used to cluster groups of energy depositions derives directly from the heavily-cited work of Ester, Kriegel, Sander, and $\mathrm{Xu}^{9}$, "Density Based Spatial Clustering of Applications with Noise (DBSCAN)". The twodimensional (2D) vertex, or more accurately "line-endpoint", finding algorithms follow a corner finding technique ${ }^{10}$ used for locating edges and corners in photographic images. Initial particle tracking employs Hough line finding techniques. ${ }^{11}$

\subsubsection{Reconstruction status}

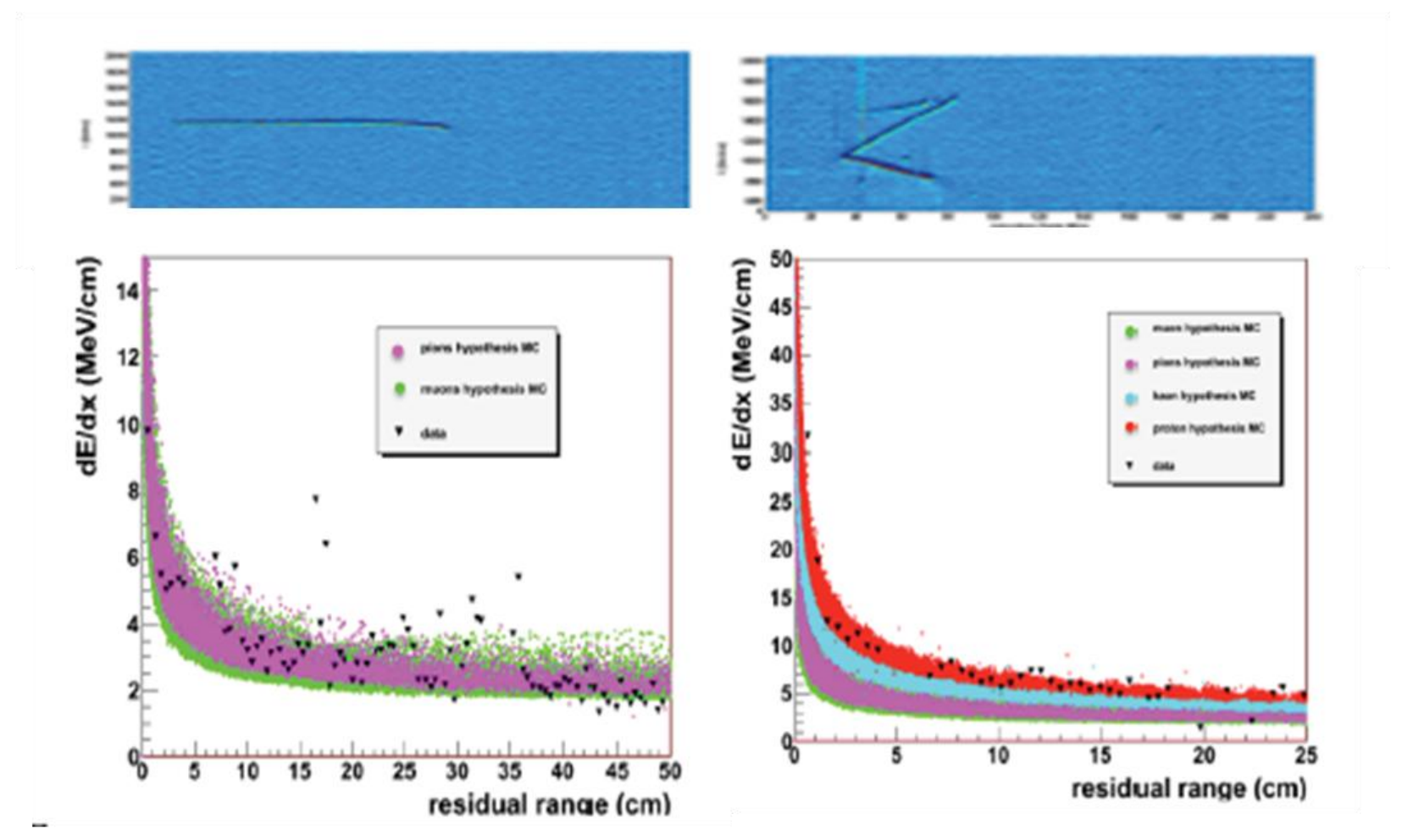

Figure 12.5 Top: ArgoNeuT Induction plane views of two events. Bottom: the dE/dx of the hits in chosen tracks in the two events. The likely muon track of the upper left event has the dE/dx of its hits (black points) calculated and superimposed over the MC $\mathrm{dE} / \mathrm{dx}$ hypotheses of pions (fuchsia) and muons (green) in the lower left image. The likely proton track at the bottom of the upper right CCQE event image has the dE/dx of its hits superimposed that for MC hypotheses of proton (red), kaon (cyan), pions (fuchsia) and muon (green).

The MicroBooNE detector intrinsically measures objects in three two-dimensional (2D) plans, $\mathrm{XZ}, \mathrm{XU}$, and XV. Physical tracks, showers, and vertices possess inherent three-dimensional (3D) attributes. The reconstruction step (iv) given above marks the point in the reconstruction chain where $2 \mathrm{D}$ wire plane projections information come together to reconstruct 3D objects. Currently the $2 \mathrm{D}$ reconstruction part of the code exists in a more mature state than the $3 \mathrm{D}$ reconstruction part. The following sections summarize the current state of the most important components of the reconstruction code. Figure 12.5 gives one indication of the current capabilities of LArSoft using real data from ArgoNeuT. Figure 12.6 and Figure 12.7 show an example of a fully reconstructed simulated event using the MicroBooNE implementation of LArSoft. 

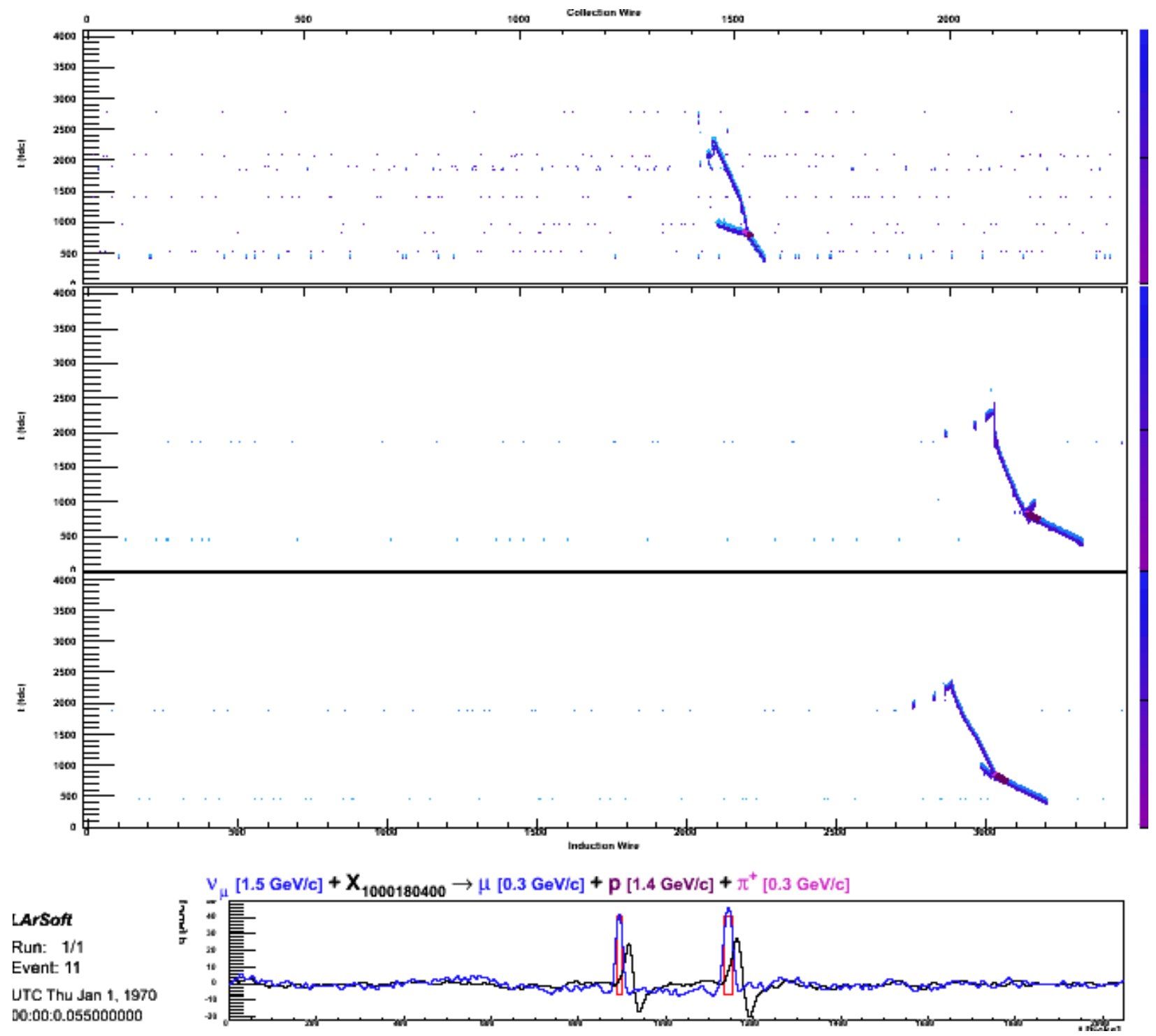

Figure 12.6 A LArSoft-simulated $v_{\mu} \mathrm{Ar} \rightarrow \mu^{-} \mathrm{p} \pi^{+}$event in the MicroBooNE detector. The vertical axes on the top three plots show TDC counts in units of $0.5 \mu$ s ticks. The horizontal axis for these plots shows the wire number for the three separate readout planes. The top plot of these three shows the collection plane view, and the middle and lower plots show the two separate induction views. The color scale on these plots indicates the ADC value for each readout. The smaller plot at bottom right shows two distinct pulses caused by the pion and muon ionization on the first induction plane for wire 3000. The underlying MC truth information appears as the bipolar trace rendered in black. The deconvolved single peak calibrated signals appear in blue, and the red boxes indicating the two hits found by hitfinder package. 


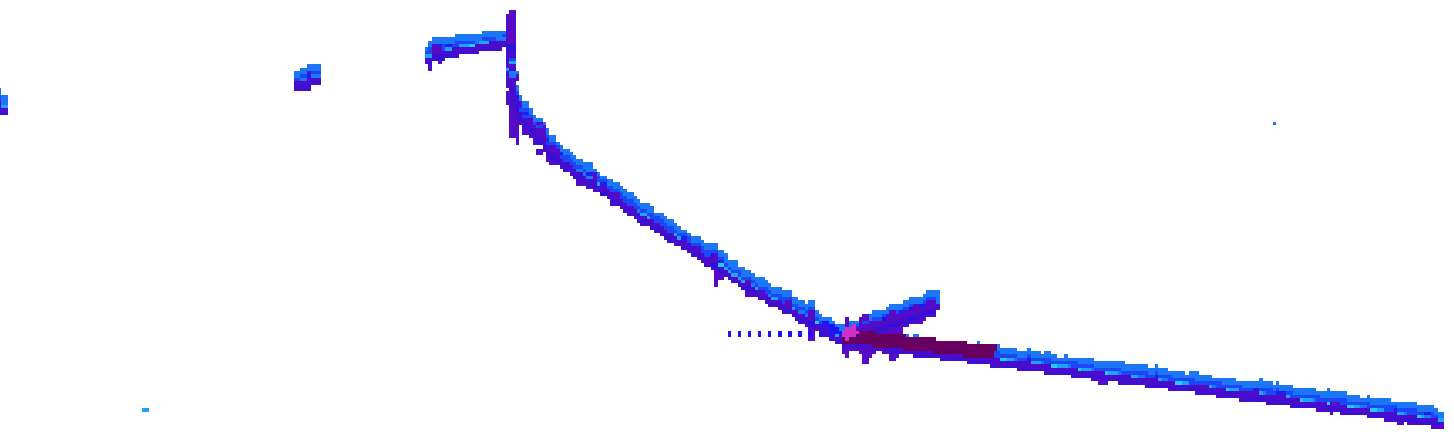

Figure 12.7 A "zoomed-in" view of the middle induction view of the A LArSoft-simulated $v_{\mu} A r \rightarrow \mu p \pi^{+}$event in the MicroBooNE detector shown in Figure 12.6. The dotted line indicates the incident neutrino coming in from the left in the figure. The muon track, with its decay electron appears in the upper left. The short track is the proton, and the longer track to the right the $\pi^{+}$.

\subsubsection{Clustering}

The DBSCAN12 procedure constructs clusters. DBSCAN, as implemented in LArSoft, accepts three parameters, a minimum cluster size MinPts and two characteristic clustering scales $\sum_{1}$ and $\Sigma_{2}, \quad$ and then executes an iterative algorithm. This begins with a "seed" hit that has not already been associated with a cluster or discarded as noise. All other non-associated hits that lie within the boundary of an ellipse with axes $\sum_{1}$ and $\sum_{2}$ centered on the seed hit add to the cluster candidate. Each hit added to the candidate cluster then acts a new seed to search for further hits. The procedure continues until no further hits join to the cluster candidate. If the total number of hits exceeds the threshold MinPts, then the cluster candidate becomes a cluster object, and its hits do not participate in further clustering. Otherwise the initial seed hit is discarded as noise and removed from the clustering sample. Once all hits have been either drawn into clusters or discarded as noise, the algorithm terminates.
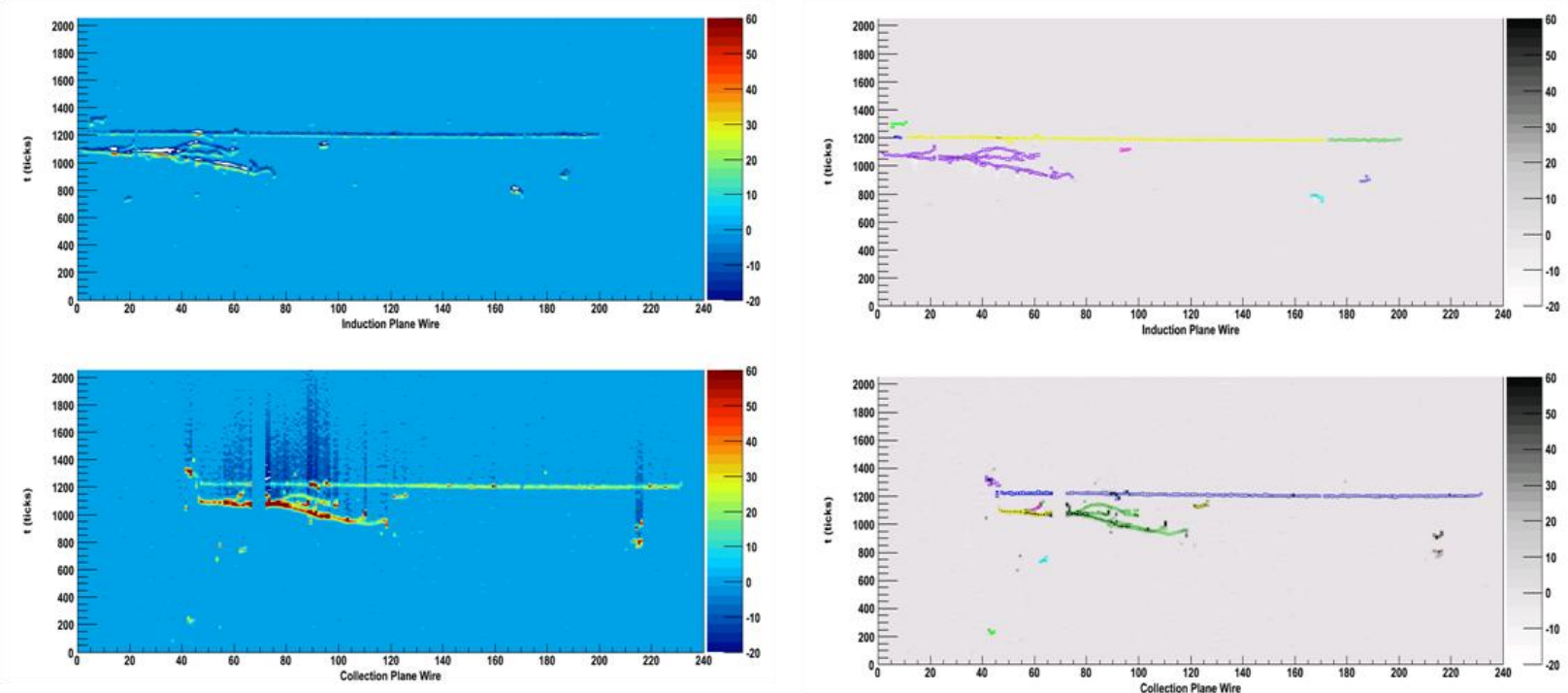

Figure 12.8 Un-clustered(left) and clustered(right) hits in the event display. 


\subsubsection{Two Dimensional Tracking}

Two dimensional tracking follows a Hough line finding method ${ }^{13,14}$. The Hough space spans two dimension identified by $(\rho, \theta)$. Each point in the Hough space represents a straight line in real space with slope angle $\theta$ and distance $\rho$ from the origin to the line. A point in real space becomes a curve in Hough space representing all possible straight lines that could pass through the point. Points lying on a line in real space will generate a family of curves in Hough space that intersect at a common $(\rho, \theta)$, as indicated in Figure 12.9.

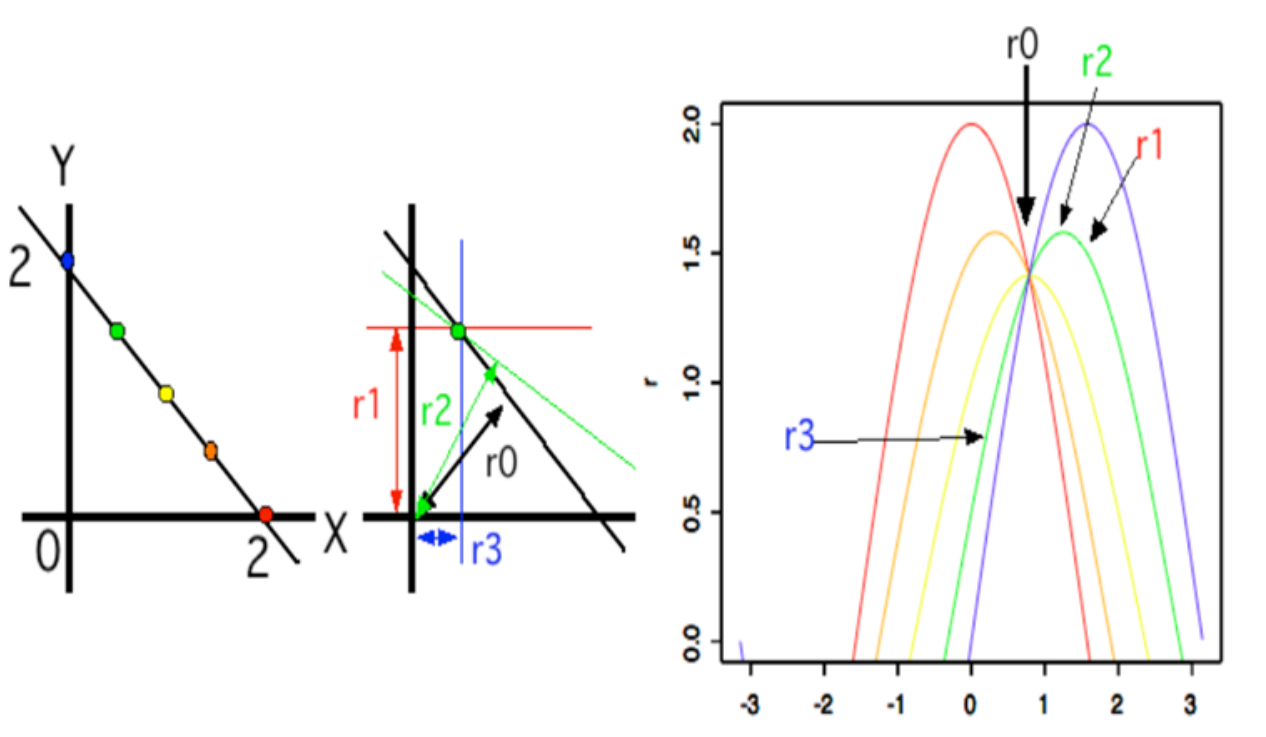

Figure 12.9 Schematic representation of the Hough line determination method.

The Hough line finder method converts all 2D hits contained in a cluster into Hough curves. The resulting $(\rho, \theta)$ space fills with many curves, with the density of the curves varying across the space. The points with the highest density of curves represent the straight line candidate with the most good passes through all the hits in a cluster. A Gaussian smoothing procedure applied to the Hough space curve density provides robustness against random fluctuations in the density and usually results in unique solutions. Hough lines candidates must pass a critical hit density threshold to be counted, and several lines can be generated per cluster up to a preset maximum line count. The resolution in each of the $\rho$ and $\theta$ dimensions for the algorithm can also be specified.

\subsubsection{Vertexing and Vertex Matching}

Defining the precise endpoints of tracks is not easy with a simple clustering algorithm like DBSCAN, or with a Hough line finding method. Hence LArSoft incorporates specialized endpoint finding algorithms. A Harris vertex finding algorithm ${ }^{15}{ }^{16}$ finds $2 \mathrm{D}$ vertices from the hits that form DBSCAN clusters. The algorithm begins with a two dimensional set of hits extracted from DBSCAN clusters. A Gaussian derivative determines the gradient of the hit density at each pixel in the 2D view of hits. A Harris vertex then becomes defined as a region with a strongly falling gradient of hit density in two orthogonal directions (forming a "corner"). The strength of the vertex follows from the magnitude of the hit density gradients. If several vertices are found within some user defined distance window, the algorithm discards all but the 
strongest vertex. This algorithm identifies a set of spatial regions that form vertex candidates independently of line finding process.

A procedure then follows to establish whether Hough lines match to Harris vertices. If a vertex exists within a user defined window around the endpoint of a reconstructed Hough line, the track becomes matched to that vertex. A Harris vertex with two or more matched Hough lines becomes a "strong" vertex. A strong vertex acquires a strength parameter equal to its Harris vertex strength multiplied by the lengths of its associated Hough lines.

An algorithm to find 3D vertices defined by intersecting tracks or prongs that point back to a common 3D point to within some pointing error exists in prototype form, but refinement of this procedure remains an outstanding task.
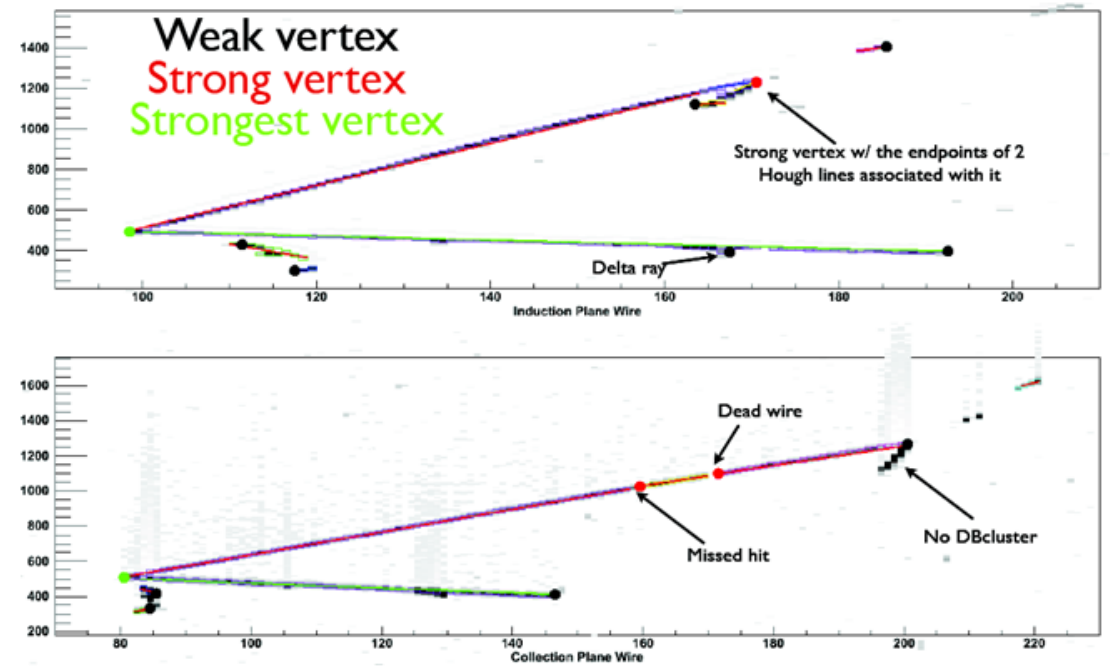

Figure 12.10 The 2D Harris vertexing method in LArSoft.

\subsubsection{Three-Dimensional Tracking}

Three-dimensional tracking remains under intense development. Here one looks to see whether Hough lines from the 2D clusters found in the individual TPC wire planes can be matched and interpreted as projections from a real 3D track in the TPC. Both LArSoft-developed and imported Kalman filter-based tracking package exists in LArSoft in a fairly mature state. The Kalman filter package has been tested successfully with ArgoNeuT data. Its estimation of track parameter covariance matrices, with inclusion of multiple Coulomb scattering processes, allow for better resolution on track parameters and a scattering-based estimate of track momentum, as has been demonstrated at ICARUS ${ }^{17}$. Kalman tracks with their pointing errors provide good inputs for the $3 \mathrm{D}$ vertexing algorithm mentioned above.

\subsubsection{Shower Finding}

Shower finding involves seeking the strongest Harris vertices in an event and counting hits within a cone (or in two dimensions, a triangle) of user specified dimensions pointing out of the vertex. If the number of found hits satisfying quality criteria exceeds a threshold, the structure becomes a shower. The functionality resembles many standard high energy collider experiment 
shower algorithms ${ }^{18}$. A working model shower finding algorithm exists, but remains under development. The current implementation is two dimensional, and it can find showers in each view individually.

Work on shower finding continues. Once 3D vertex finding becomes mature, the development and optimization of a 3D fixed cone shower finding algorithm can follow with high priority. Further progress entails developing criteria to automatically distinguish electromagnetic showers from tracks produced by protons, muons, and electrons. This can presently be performed only through hand-scanning.

\subsubsection{Calorimetry}

Calorimetry can be implemented by straightforwardly looping over prongs (tracks or showers) and summing up the properly-calibrated signals of each hit in each prong. Corrections must be applied for the prong's angle with respect to the wire in TPC plane, and for saturation effects in energy loss for slow particles (Birk's law). This work is largely done. Particle identification will follow from the per-track $\mathrm{dE} / \mathrm{dx}$ determination that results from the calorimetry.

\subsubsection{Optical Reconstruction Techniques}

At this stage, no optical reconstruction code works within the framework of LArSoft, but once an operational TPC-based 3D reconstruction chain exists, scintillation-timing-based methods can be implemented to complement $\mathrm{dE} / \mathrm{dx}$-based particle identification determination. As detailed in Section 12.4.3.4, liquid argon scintillates with two characteristic time constants, and the light yield of each depends on the $\mathrm{dE} / \mathrm{dx}$ of the originating track. For certain events the time profile of detected light may offer the ability to enhance particle identification capabilities, above those provided by TPC information alone. Development continues on two algorithms.

\subsubsection{Pulse shape discrimination (PSD)}

The PSD technique has been pioneered by dark matter experiments ${ }^{19,20}$ to separate keV nuclear recoils from $\mathrm{keV}$ electron recoils in liquid argon detectors. This method involves simply taking the ratio of light in the first $100 \mathrm{~ns}$ after the interaction to the complete light collected in an event. Particles with a higher $\mathrm{dE} / \mathrm{dx}$ have the slow component of their scintillation light more strongly quenched, and so this ratio acts as a discriminator between types of particles in single track events. 


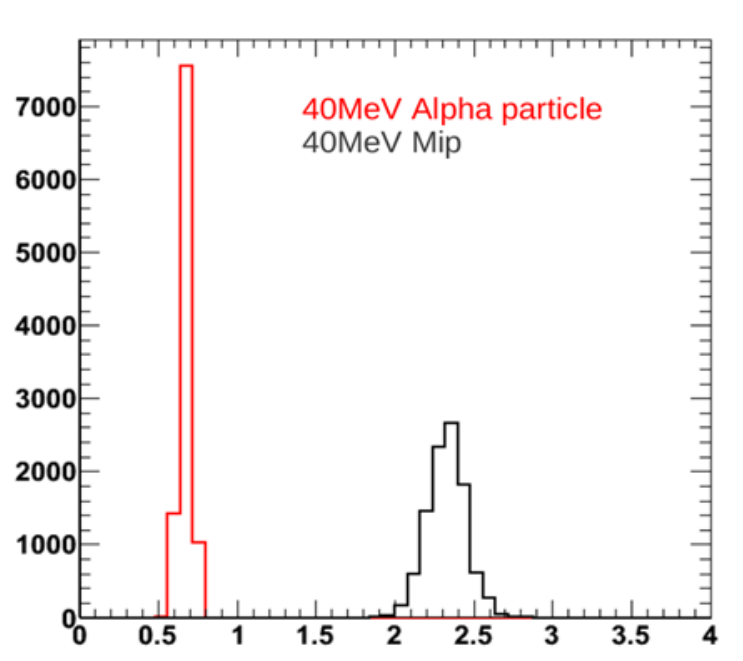

Figure 12.11 Ratio of late light to prompt light for $40 \mathrm{MeV}$ alpha particles and minimum ionizing particles in MicroBooNE.

Compared to a LAr-based dark matter detector, MicroBooNE neutrino events produce tracks of much higher in energy, and which consequently experience less quenching of their slow component. Further, the relatively low photocathode coverage of MicroBooNE results in a somewhat sparse sampling of the light. Nevertheless, detailed simulations indicate that useful discriminating power exists. Figure 12.11 shows the value of the late to prompt light collection ratio for a $40 \mathrm{MeV}$ alpha particle and a $40 \mathrm{MeV}$ minimum ionizing particle. Prompt and late light are defined as being the integral of the PMT pulse for the first $100 \mathrm{~ns}$, and from $100 \mathrm{~ns}$ to the end of the sample frame, respectively. The optical reconstruction technique shows a clear difference. The TPC would have difficulty in reconstructing tracks produced by such low energy particles.

\subsubsection{Pulse Shape Augmented Reconstruction (PSAR)}

Pulse height discrimination can only be used in the form described above for events with very simple topologies. However, optical pulse height information can be used to augment TPCbased particle identification in more complex events using a method under development pulse shape augmented reconstruction (PSAR). This could be particularly useful in classes of events with ambiguous charge-based $\mathrm{dE} / \mathrm{dx}$ particle identification.

The PSAR algorithm consists of using track parameter information determined from the TPC. The expected scintillation production map in space and time will quickly be produced for each particle identification hypothesis in the event. By comparing the measured time profile of the light detected at each PMT in the system to the fast simulation expectation for each hypothesis, an independent measure of the relative likelihood of each particle identification hypothesis can be obtained and compared to the charged-based relative likelihoods.

\subsubsection{Event building}

The event building tool must be able to compartmentalize sub-events within a particular time window. For example, a stopping cosmic muon track with its Michel electron track and vertex 
must be separated from a beam window-triggered neutrino event with its detector objects. The event building tool remains under development.

\subsection{Parallel Processing}

The Fermilab CD supports computer farm processing for LArSoft users through the condor tool. The condor job submitter and node allocator can be accessed by a well-defined prescription that documented in detail on the LArSoft central redmine website ${ }^{21}$. Currently about 100 worker nodes in the FNAL intensity frontier cluster are available; a few thousand more nodes on the wider FNAL grid can also be accessed. This should provide ample computing as MicroBooNE enters a phase of high statistics Monte Carlo event generation and reconstruction.

\subsection{Collaboration Productivity}

New LArSoft users and developers can be quickly added by MicroBooNE software managers using simple scripts that currently give access to a relevant computing node, uboonegpvm0N, and to the svn repository. Access to the condor batch system requires a CD service ticket submission following explicit directions linked from the top level LArSoft website.

1 The GENIE Collaboration. GENIE Object Oriented Neutrino Event Generator.

http://projects.hepforge.org/genie/. arXiv:0905.2517.

2 http://nuclear.llnl.gov/simulation/.

3 NIM A 506, 250-303 (2003).

4 Chytracek, R. and McCormick, J. and Pokorski, W. and Santin, G., Geometry Description Markup Language for Physics Simulation and Analysis Applications, IEEE Trans. Nucl. Sci. (2006), pp. 2892-2896.

5 Chris Hagmann, David Lange, and DougWright. Cosmic Ray Shower Library (CRY). Technical Report UCRLTM-229453, LLNL, 2008. http://nuclear.llnl.gov/simulation/cry.pdf.

6 S. Agostinelli et al. GEANT4: A simulation toolkit. Nucl. Instrum. Meth., A506:250-303, 2003.

7 W. Walkowiak, NIM A 449 (2000) 288-294.

8 Nucl.Instrum.Meth.A523:275-286,2004.

9 Ester, Kriegel, Sander, \& Xu, Proceedings of Second International Conference on Knowledge Discovery and Data Mining, Portland, OR (1996).

10 JINST 5, P07006 (2010).

11 Queisser, A. "Computing the Hough Transform", C/C++ Users Journal 21, 12 (Dec. 2003), Niblack, W. and Petkovic, D. "On Improving the Accuracy of the Hough Transform", Machine Vision and Applications 3, 87 (1990).

12 M. Ester, H.-P. Kriegel, J. Sander, X. Xu, A density-based algorithm for discovering clusters in large spatial databases with noise, in: Proceedings of Second International Conference on Knowledge Discovery and Data Mining, Portland, OR, 1996, pp. 226-231.

13 Queisser, A. "Computing the Hough Transform", C/C++ Users Journal 21, 12 (Dec. 2003)

14 Niblack, W. and Petkovic, D. On Improving the Accuracy of the Hough Transform", Machine Vision and Applications 3, 87 (1990). 
15 C. Harris and M. Stephens (1988). "A combined corner and edge detector". Proceedings of the 4th Alvey, Vision Conference. pp. 147-151.

16 B. Morgan (2010). "Interest Point Detection for Reconstruction in High Granularity Tracking Detectors". arXiv:1006.3012v1 [physics.ins-det].

18 A. Ankowski et al., "Measurement of Through-Going Particle Momentum By Means Of Multiple Scattering With The ICARUS T600 TPC," Eur.Phys.J.C48:667-676, 2006.

${ }^{18}$ Fermilab-Conf 90-249/E, "Toward a Standardization of Jet Definitions".

19 A. Hime. DEAP \& CLEAN detectors for low energy particle astrophysics. AIP Conf. Proc., 870:205-207, 2006.

20 W. H. Lippincott et al. Scintillation time dependence and pulse shape discrimination in liquid argon. Phys. Rev., C78:035801, 2008.

${ }^{21}$ https://cdcvs.fnal.gov/redmine/projects/larsoftsvn. 


\section{MicroBooNE Collaboration}

\subsection{Brookhaven National Laboratory}

- Hucheng Chen

- Susan Duffin

- Jason Farrell

- Francesco Lanni

- Yichen Li

- David Lissauer

- George Mahler

- Don Makowiecki

- Joseph Mead

- Veljko Radeka

- Sergio Rescia

- Andres Ruga

- Jack Sondericker

- Craig Thorn (Deputy Project Manager)

- Kuo-Chen Wu

- Bo Yu

\subsection{Columbia University}

- Leslie Camilleri

- Rachel Carr

- Gary Cheng

- Georgia Karagiorgi

- Camillo Mariani

- Bill Seligman

- Mike Shaevitz

- Bill Willis

- Bill Sippach

- Cheng-Yi Chi

\subsection{Fermilab}

- Bruce Baller

- Larry Bartoszek

- Herbert Greenlee

- Cat James (Deputy Project Manager)

- Hans Jostlein

- Michael Kirby

- Stephen Pordes

- Gina Rameika (Project Manager) 
- Jennifer Raaf

- Brian Rebel

- Rich Schmitt

- Dave Schmitz

- Jin-Yuan Wu

- Sam Zeller

\subsection{Kansas State University}

- Tim Bolton

- Saima Farooq

- David McKee

- Glenn Horton-Smith

\subsection{Los Alamos National Laboratory}

- Gerry Garvey

- Jackie Gonzales

- Bill Louis

- Chris Mauger

- Geoff Mills

- Zarko Pavlovic

- Richard Van de Water

- Hywel White

\subsection{Massachusetts Institute of Technology}

- William Barletta

- Len Bugel

- Janet M. Conrad

- Christina Ignarra

- Ben Jones

- Teppei Katori

- Tess Smidt

- Arati Prakash

\subsection{Michigan State University}

- Carl Bromberg

- Dan Edmunds

\subsection{Princeton University}

- Kirk McDonald

- William R. Sands 


\subsection{Saint Mary's University of Minnesota}

- Paul Nienaber

\subsection{Syracuse University}

- Mitch Soderberg

\subsection{University of Cincinnati}

- Randy Johnson (Project Scientist)

\subsection{University of Texas at Austin}

- Sacha Kopp

- Karol Lang

- Rashid Mehdiyev

\subsection{Yale University}

- Flavio Cavanna

- Eric Church

- Bonnie T. Fleming (Spokesperson)

- Roxanne Guenette

- Ellen Klein

- Joseph Lozier

- Ornella Palamara

- Kinga Partyka

- Adam Patch

- Josh Spitz

- Andrzej Szelc 